\title{
EPSM 2020, Engineering and Physical Sciences in Medicine
}

\section{Oral Abstracts: 0001-0088}

Monday $28^{\text {th }}$ October

- Plenary

- Education \& Professional Standards

- Radiotherapy quality assurance processes

- Diagnostic and interventional imaging

- Diagnostic and interventional imaging

- Radiotherapy treatment planning and outcomes

- Image segmentation planning and outcomes

- Patient specific quality assurance

- Radiotherapy dose modelling

- Radiotherapy image guidance

Tuesday $29^{\text {th }}$ October

- Plenary

- Nuclear Medicine

- SRS and SABR techniques

- Image reconstruction and CBCT

- Radiation dosimetry

- MRI in radiotherapy

- Radiotherapy treatment delivery and equipment

- 3D printing and phantoms

- Film, gel and other detectors

Poster Abstracts: P001-P050

- Biomedical Engineering

- Clinical Engineering

- Education and Professional Standards

- Radiation Biology

- ROMP

- Brachytherapy

- Dosimetry

- New Technologies and Techniques

- Quality Assurance

- Stereotactic radiotherapy \& Radiosurgery

- Treatment Planning

- Radiation Protection

- Radiology Physics

- Reflection and Projection

\section{- Other}

\section{O001 COVID-19: Has it caused a paradigm shift in teaching medical physics in the future?}

\author{
A Haworth ${ }^{1}$, A L Fielding ${ }^{2}, S$ Marsh $^{3}$, P Rowshanfarzad ${ }^{4}$,

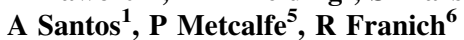 \\ ${ }^{1}$ School of Physics, University of Sydney, Australia \\ (annette.haworth@sydney.edu.au [Presenting author]). ${ }^{2}$ Science \& \\ Engineering Faculty, Queensland University of Technology, \\ Brisbane, Australia (a.fielding@qut.edu.au). ${ }^{3}$ School of Physical \\ and Chemical Sciences, University of Canterbury, New Zealand \\ (steven.marsh@ canterbury.ac.nz). ${ }^{4}$ Department of Physics, \\ University of Western Australia, Australia \\ (pejman.rowshanfarzad@uwa.edu.au). ${ }^{5}$ School of Physical Sciences, \\ University of Adelaide, Australia \\ (alexandre.santos@adelaide.edu.au). ${ }^{6}$ Centre for Medical Radiation \\ Physics, University of Wollongong, Australia \\ (metcalfe@uow.edu.au). ${ }^{7}$ School of Science, RMIT University, \\ Australia (rick.franich@rmit.edu.au)
}

Introduction The Australian Institute of Physics (AIP) recently released a position statement regarding replacement of face-to-face (F2F) classes by online delivery in physics courses impacted by the COVID-19 pandemic [1]. It concluded online teaching should only be viewed as a short-term measure during an emergency situation. The arguments presented against online teaching were related to preserving the excellent reputation of Australian physics teaching which is based on a) the largely F2F teaching methods and hands-on curriculum b) high levels of student-student and teacher-student interaction and c) invigilated examinations. The seven course coordinators from Australia and New Zealand's (ANZCCs) ACPSEM accredited postgraduate programs considered the relevance of these arguments to medical physics education.

ANZ Medical Physics Courses All six universities offering coursework (rather than research)-based degrees transitioned from F2F teaching to online in a period of a few days to $<2$ weeks. The ANZCCs held monthly meetings to provide mutual support. Most ANZCCs reported good, if not better attendance at online lectures compared with traditional F2F lectures. Engagement in lectures 
improved as students became familiar with the technology. The impact on student-student interaction could not be assessed but is assumed to be hindered due to the lockdown occurring early in the semester. Limited experience in conducting online exams resulted in a steep learning curve to identify methods to assure academic integrity. Hospital-based practicals were either cancelled, postponed or replaced with virtual teaching alternatives. Students conducting hospital-based research projects either suspended or redesigned projects to enable remote completion.

Conclusions The ANZCCs agreed that whilst online teaching could offer new opportunities, F2F teaching has significant advantages, and the hospital experience is essential for our vocational-style courses. The clinical community is urged to identify methods to facilitate the early return of hospital-based teaching to prepare future TEAP registrars with the appropriate foundational education.

Reference https://aip.org.au/wp-content/uploads/2020/05/2020_05_ 01_PositionStatementRePhysicsTeachingInclExecSummary-FINAL2.p.

\section{O002 Continuing ROMP TEAP examinations during the COVID-19 pandemic, including Written, Part A and Part B exams}

\section{Dempsey ${ }^{1}$, T Ackerly², C M Thompson ${ }^{3}$}

Australasian College of Physical Scientists and Engineers in Medicine, Sydney, Australia; Calvary Mater Newcastle Hospital, Newcastle, Australia (claire.dempsey@acpsem.org.au [Presenting author]). ${ }^{2}$ Alfred Health, Melbourne, Australia (T.Ackerly@alfred.org.au). ${ }^{3}$ Auckland District Health Board, Auckland, New Zealand (ChristineT@adhb.govt.nz)

Introduction The COVID-19 pandemic has impacted Radiation Oncology clinical departments in a variety of ways, each of these having a flow-on effect to Radiation Oncology Medical Physics (ROMP) training, education and assessment program (TEAP) registrars. The ACPSEM made the decision to put measures in place to continue the external assessment of registrars without compromising the quality of the assessment, the safety of the registrar and examiner (and their respective departments) and abiding by various state/national COVID-19 restrictions.

Method An alternative method for Part A and B examinations needed to allow for examiner/registrar interaction and visualisation of practical equipment setup as well as supervision of the exam to ensure appropriate examination techniques were followed. Zoom video conferencing was used for Part A and B examinations with two examiners remotely assessing the registrar with a third examiner (local to the department) on-site to ensure exam processes were followed without being involved in the grading of the candidate.

There were also concessions for the written examination. Candidates not able to complete the brachytherapy competencies due to COVID19 restrictions were given the opportunity to undertake the other core module written exam questions, with the brachytherapy module being completed at a later date.

Results To date, 15 registrars have been able to successfully complete TEAP final exams despite COVID-19 restrictions. Post-exam evaluations provided by both examiners and registrars indicated that the Zoom examination method was fair and the examination standard was maintained.

To date, 1 registrar has undertaken the written exam without having completed the required brachytherapy competencies. This registrar is hoping that restrictions will ease and allow them to finish the outstanding brachytherapy competencies so that they can complete the brachytherapy written exam questions in March 2021.
Conclusion Despite the challenges COVID-19 has placed on TEAP registrars, the ACPSEM has adjusted its examination methodologies to continue to allow registrars to progress through the program.

References/Acknowledgements The authors acknowledge the flexibility of the examiners, candidates and their departments, who were prepared to use these alternative methods.

\section{O003 Simulation and problem-based learning: engaging medical physics students in active and authentic learning}

\author{
A L Fielding ${ }^{1}$, H Huijser ${ }^{1}$, S Kickbusch ${ }^{1}$, P Anand ${ }^{2}$, I Czaplinski ${ }^{1}$ \\ ${ }^{1}$ Science \& Engineering Faculty, Queensland University \\ of Technology (QUT), Brisbane, Australia (a.fielding@qut.edu.au \\ [Presenting Author]). ${ }^{2}$ Learning and Teaching Unit, Queensland \\ University of Technology (QUT), Brisbane, Australia \\ (pranit.anand@qut.edu.au)
}

Introduction We report the results of a project to investigate if replacing traditional lectures in a radiotherapy unit with simulation and problem-solving activities could increase student engagement and enhance student knowledge and understanding of complex radiotherapy physics concepts.

Method The first of two new activities involved the students using a BEAMnrc/DOSXYZnrc Monte-Carlo model of a linear accelerator (LINAC) and water phantom. The activity aimed to give students hands on experience of the production of $\mathrm{x}$-rays and the role of the different components of the LINAC on the clinical beam as well the resulting dosimetric data. The second activity involved using the matRAD inverse treatment planning software to perform comparative planning of protons vs photon IMRT two clinical cases. The aim was to give the students hands on experience of inverse planning optimisation as well as an understanding of the potential advantages of protons over photons. A qualitative mixed methods approach was used to explore student experiences with the unit. Class observations, student feedback through anonymous online surveys, focus groups and staff reflections were used to gain an in-depth understanding of the way in which students engaged with the new activities in the unit. Results Overall the results indicate that the approach was well received by the students who valued the opportunity to use sophisticated software to simulate two different aspects of clinical radiation therapy. Although there were obvious challenges associated with using potentially resource-hungry software on students' standard laptops, there was an observed improvement in student engagement with subject content as well as engagement with the instructor and between peers.

Conclusion Replacing traditional face to face lectures with clinically relevant hands on simulation and problem-solving activities in a postgraduate medical physics course was found to increase student engagement with subject content as well as engagement with instructors and between peers.

O004 The role of Diagnostic Imaging Medical Physicists in supporting clinical policy change and the implementation of evidence-based clinical practice

\section{E Ryan', D Dinham ${ }^{1}$, K Norynberg ${ }^{2}$}

${ }^{1}$ Biomedical Technology Services, Health Support Queensland, Queensland Health, QLD, Australia (elaine.ryan@ @ealth.qld.gov.au, Presenting author), (dalia.dinham@ health.qld.gov.au). ${ }^{2}$ Department 
of Medical Imaging and Nuclear Medicine, Queensland Children's Hospital, Brisbane, Australia (kerrie.norynberg@health.qld.gov.au)

Introduction Traditionally medical physics service support within Diagnostic Imaging departments has been thought of as the provision of radiation safety services and fulfilling regulatory requirements concerning equipment compliance. The aim of this paper is to illustrate, using a clinical example of the cessation of the use of paediatric gonadal shielding, that the implementation of a major clinical policy change requires medical physics input at all stages through the process. Following the publication in the literature of a number of position statements supporting the termination of the use of gonadal shielding in paediatric pelvic imaging there was a substantial body of work required to implement a departmental policy change. Medical physicists were needed in order to ensure scientific rigour and to provide essential scientific support.

Method Evidence and examples of scientific input from medical physics staff was provided in all aspects of changing the use of gonadal shielding within the department: 1. Providing a background review of the literature and current evidence 2. A review of current practice within the department 3 . Consultation with radiologists and radiographers 3. updating the Radiation Safety and Protection Plan 4. Provision of education to all staff involved in imaging of paediatric patients 5. Development of support materials to be provided to patients/parents/carers to explain the reasoning behind the policy change 6. Formulation of a research protocol (including ethics submission and approval) to audit the process 7 . Analysis of results 8 . Communication to the wider imaging community.

Results and Conclusion This reflection has shown that through this process the contribution of Diagnostic Imaging Medical Physicists is invaluable in ensuring that medical imaging services are operating to the highest standard of evidence-based practice. Sharing this example shows the impact of a DIMP service to the clinical department.

\section{Assessment of adaptive radiotherapy workflows for head and neck cancer}

\author{
George Antoniou ${ }^{1}$, Scott Penfold ${ }^{2}$ \\ ${ }^{1}$ Department of Physics, University of Adelaide, South Australia \\ (george.antoniou@adelaide.edu.au, Presenting author). Department \\ of Medical Physics, Royal Adelaide Hospital, South Australia \\ (scott.penfold@sa.gov.au)
}

Introduction Inter-fractional anatomical variations in head and neck cancer patients can lead to clinically significant dosimetric changes. Adaptive re-planning should thus commence in order to negate any potential over-dosage to organs-at-risk (OAR), as well as potential under-dosage to target lesions. The aim of this study is to explore the correlation between transit fluence, as measured at an electronic portal imaging device (EPID), and DVH metrics to target and OAR structures. This work will guide implementation of transit EPID dosimetry as a decision support metric for head and neck adaptive radiotherapy.

Method Ethics approval has been obtained to conduct a retrospective analysis of 20 patients who have completed a course of radiotherapy at the RAH with at least one re-plan. The purpose of the study is to investigate the correlation between change in transit radiation fluence and change in key internal dosimetric parameters. An in-house developed RayStation script will allow for transit fluence simulation on planning CTs. The RayStation script was validated by comparison to EPID measured transit dosimetry fields using a phantom on a TrueBeam linac. A modified gamma analysis tool was developed to quantify changes in transit fluence with changes in phantom geometry.
$X$ and $Y$ Profiles of Gamma Analysis Between Full Phantom and Reduced Phantom Images
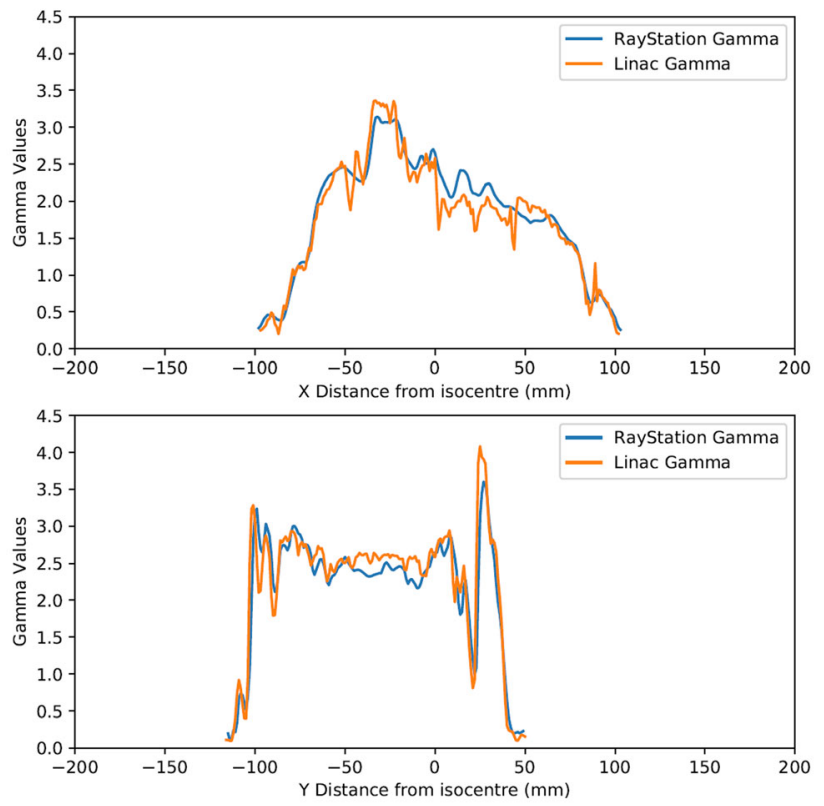

Figure $1 \mathrm{X}$ - (top) and Y-Profiles (bottom) of (2\%, $2 \mathrm{~mm})$ gamma analysis tests on change in predicted (blue) and measured (orange) transit dosimetry

Results A comparison of linac measured change in transit fluence and RayStation calculated change in transit fluence, when a solid water phantom is reduced in thickness $(1 \mathrm{~cm}$ removed anteriorly and posteriorly), is shown in Fig. 1. A gamma analysis was performed on images acquired with a full phantom and a reduced phantom thickness. Figure 1 indicates that the change in RayStation transit fluence reflects change in true transit fluence as measured on a linac.

Conclusion The RayStation transit dosimetry script developed was validated against measured data. The tool can thus be utilised to explore the correlation between transit fluence and DVH metrics to target and OAR structures in future work.

\section{O006 Timely review of electronic portal images in a multicentre clinical trial of deep inhalation breath hold in breast cancer patients}

\section{T Kron ${ }^{1,2}$, P Lonski ${ }^{3}$, J Lehmann ${ }^{4}$}

${ }^{1}$ Peter MacCallum Cancer Centre and Sir Peter MacCallum Department of Oncology of the University of Melbourne, Melbourne, VIC 3000, Australia. ${ }^{2}$ Centre of Medical Radiation Physics, University of Wollongong, NSW 2500, Australia.

(Tomas.Kron@petermac.org, [Presenting author]). ${ }^{3}$ Department of Physical Sciences, Peter MacCallum Cancer Centre, Melbourne, VIC 3000, Australia. (Peta.Lonski@ petermac.org). ${ }^{4}$ Newcastle Calvary Mater Hospital, Newcastle, NSW 2300, Australia. (Joerg.Lehmann@calvarymater.org.au)

Introduction Deep inhalation breath hold (DIBH) is considered a good method to reduce heart dose in left-sided breast cancer patients undergoing radiotherapy. The HART trial (TROG 14.04) aimed at assessing this in a multicentre setting with one of the endpoints being reproducibility of breath hold position. 
Method The trial accrued 32 patients with left sided breast cancer in six contributing centres in Australasia. DIBH was controlled using Varian RPM or Elekta ABC devices. Reproducibility of breath hold was assessed during the first week of treatment using daily electronic portal imaging (EPI). The images were uploaded to the central quality management system of the TransTasman Radiation Oncology Group (TROG) and reviewed by an independent physicists using mid lung distance (MLD) as a surrogate for patient positioning. Reviews were conducted using MIM software and many of the images were reviewed again after completion of the trial by an independent observer together with additional images acquired over the remainder of the treatment.

Results Two of the 32 left sided breast patients accrued did not proceed to DIBH (one clinician and one patient decision). Image review for the remaining 30 patients was completed within three or less working days after the first week of treatment and communicated to the treating centre. Three images of 177 were found to be of insufficient quality for review. Inter-observer reproducibility was good (mean $\mathrm{r} 2=0.734, \mathrm{n}=10$ image sets with 5 or more dual observations, Figure A). Figure B shows as comparison the MLD judged by the same observer from medial and lateral images provided for six patients (mean $\mathrm{r} 2=0.469$ ).

Conclusion Review of multiple verification images acquired during a clinical trial is feasible and provides a feedback or intervention point taking actual treatment delivery and not just the treatment plan into consideration.

Acknowledgements The help of the TROG QA team as well as the financial support of Breast Cancer Trials and the Gross Foundation is greatly appreciated.
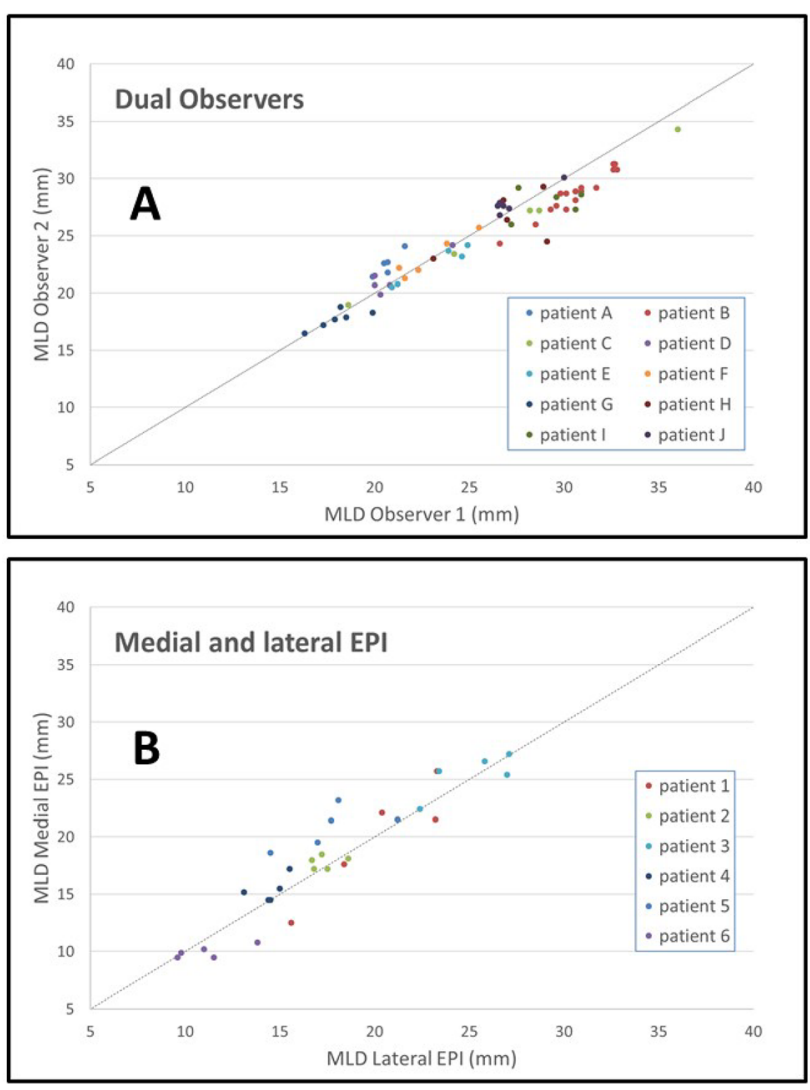

\section{A freely available standardized adaptive evaluation report template}

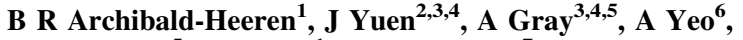 \\ S Deshpande ${ }^{5}$, M Byrne ${ }^{1}$, M G Jameson ${ }^{7}$
}

${ }^{1}$ Icon Cancer Centres, Wahroonga, NSW, Australia. (ben.archibaldheeren@icon.team), (Mikel.byrne@icon.team). ${ }^{2}$ St George Cancer Centre, Kogarah, NSW, Australia. ${ }^{3}$ Ingham Institute for Applied Medical Research, Sydney, NSW, Australia. ${ }^{4}$ South Western Sydney Clinical School, UNSW, Sydney, NSW, Australia.

(Johnson.Yuen@health.nsw.gov.au). ${ }^{5}$ Liverpool and Macarthur Cancer Therapy Centres, Sydney, NSW, Australia.

(Alison.gray@health.nsw.gov.au),

(Shrikant.Deshpande@ @ealth.nsw.gov.au). ${ }^{6}$ Peter MacCallum Cancer Centre, VIC, Australia. (Adam.yeo@petermac.org). ${ }^{7}$ Genesis Cancer Care, Sydney, NSW, Australia. (michael.jameson@genesiscare.com, [Presenting author])

Introduction Adaptive radiotherapy is complex, with multi-disciplinary processes and the potential for multiple deformable registrations reinforcing the need for patient specific quality assurance [1-4]. A practical and robust method for assessment, evaluation and reporting of offline adaptive cases is ideal. This study aims to characterise a standardised solution developed by the ACPSEM Medical Image Registration Special Interest Group (MIRSIG).

Method A working group was created based on an expression of interest amongst MIRSIG for the development of a quality assurance reporting template compliant with AAPM TG132 [4] recommendations for Australian and New Zealand radiotherapy sites. The template was developed by initial prototype, alpha testing of branched versions, survey of working group, and development of the consensus template with core ( $>75 \%$ agreement) and optional checks.

Results 16 survey responses were analysed to generate a consensus template to facilitate multi-disciplinary tasks and handover for Radiotherapists, Physicists, and Oncologists.

Core checks identified were divided into 4 major processes (Figure 1):

- Request (Radiotherapists): Date of Request (88\%), Patient Identifier (94\%), Rationale for Request (88\%), Fractionation (delivered/total) $(88 \%)$

1. Adaptive Process: Adaptive Methodology (94\%), Registration Information (82\%), Registration Accuracy (94\%), Accuracy of Propagated Contours (87\%), Violations of Plan Objectives (94\%), ROIs Failing objectives (81\%)

2. Adaptive Check: Overall Registration Accuracy (88\%), Regions of Unrealistic Deformation (94\%), Acceptance/ Rejection (regarding dose accuracy in process) (81\%)

3. Clinical Decision: Acceptance of Process Uncertainties (77\%), Clinical Action (100\%), Clinical Deadline (92\%).

In addition, optional checks were included to account for the unique hardware and software configurations within departments. Key areas for further development include multi-disciplinary feedback and training through webinars, correlation with deformable metrics, and integration into clinical trials.

Conclusion A template for offline adaptive checks was developed by MIRSIG through multi-institutional collaboration and will be made available on https://www.acpsem.org.au/About-the-College/SpecialInterest-Groups/MIRSIG.

Acknowledgements The ACPSEM MIRSIG acknowledges the generous support of ACPSEM, ASMIRT, TROG, RANZCR, and participating departments for making events and projects such as this possible. 


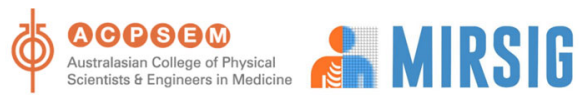

Adaptive Evaluation Report
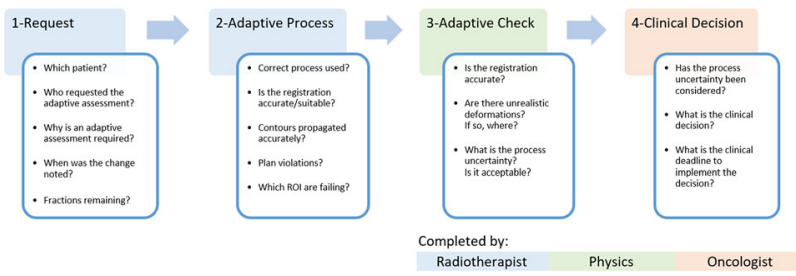

Figure 1 A schematic diagram of the MIRSIG adaptive evaluation report

\section{References}

1. Noel CE, Santanam L, Parikh PJ, Mutic S. (2014) Process-based quality management for clinical implementation of adaptive radiotherapy. 41(8):081717.

2. Brock KK. (2019) Adaptive Radiotherapy: Moving Into the Future. Seminars in Radiation Oncology. 29(3):181-184.

3. Paganelli C, Meschini G, Molinelli S, Riboldi M, Baroni G. (2018) Patient-specific validation of deformable image registration in radiation therapy: Overview and caveats. Med Phys 45(10):e908-e922.

4. Brock KK, Mutic S, McNutt TR, Li H, Kessler ML. (2017) Use of image registration and fusion algorithms and techniques in radiotherapy: Report of the AAPM Radiation Therapy Committee Task Group No. 132. Medical Physics. 44(7):e43-e76.

\section{O008 Updates to the ARPANSA Diagnostic Reference Level program}

\author{
P D Thomas ${ }^{1}$, P A Marks', A Mason ${ }^{1}$ \\ ${ }^{1}$ Medical Imaging Section, ARPANSA \\ (peter.thomas@arpansa.gov.au), (paul.marks@arpansa.gov.au \\ [Presenting author]), (alan.mason@ arpansa.gov.au)
}

Introduction ARPANSA's Diagnostic Reference Level (DRL) program supports optimisation of medical exposures by providing guidance on typical dose for common imaging procedures. Routine comparison against DRLs is a component of quality assurance and is included in regulatory requirements such as the Medical Exposure Code [1] and the Commonwealth Diagnostic Imaging Accreditation Scheme [2].

Method ARPANSA's National Diagnostic Reference Level Service (NDRLS) collects CT survey data through a web portal. Data on image-guided interventional procedures (IGIP) is collected by e-mail using spreadsheet templates. Median values of dose metrics from each survey are reported as facility reference levels (FRLs). Surveys include data for up to 20 patients in CT and 30 patients for IGIP. National DRLs are based on the third quartile of the distribution of FRLs.

Results In 2019, 4781 CT surveys from 683 scanners were completed. Third quartiles of the FRL distributions for each dose metric, categorised by scan region, are shown in Table 1 and compared with the national DRLs. The data received are consistent with the national DRLs. There is now sufficient data to establish a DRL for KUB scans. For IGIP, 47 surveys were completed in 2019. A liaison panel reviewed the data collected through to the end of 2018 and recommended the following national DRLs for diagnostic coronary
Table 1 Third quartiles of the 2019 CT FRL distributions for adult patients and comparison with the national DRLs, categorised by scan region

\begin{tabular}{|c|c|c|c|c|c|}
\hline \multirow[t]{2}{*}{ Scan Region } & \multirow[t]{2}{*}{ Surveys } & \multicolumn{2}{|c|}{$\mathrm{CTDI}_{\mathrm{vol}}(\mathrm{mGy})$} & \multicolumn{2}{|c|}{ DLP (mGy.cm) } \\
\hline & & $\begin{array}{l}\text { 3rd Quartile } \\
\text { (2019) }\end{array}$ & DRL & $\begin{array}{l}\text { 3rd Quartile } \\
\text { (2019) }\end{array}$ & DRL \\
\hline Head & 712 & 48.8 & 52 & 849 & 880 \\
\hline Cervical Spine & 565 & 20.6 & 23 & 433 & 470 \\
\hline $\begin{array}{l}\text { Soft-Tissue } \\
\text { Neck }\end{array}$ & 453 & 15.0 & 17 & 437 & 450 \\
\hline Chest & 652 & 9.0 & 10 & 356 & 390 \\
\hline $\begin{array}{l}\text { Chest-Abdo- } \\
\text { Pelvis }\end{array}$ & 559 & 11.3 & 11 & 852 & 940 \\
\hline Abdo-Pelvis & 676 & 11.4 & 13 & 558 & 600 \\
\hline $\begin{array}{l}\text { Kidney-Ureter- } \\
\text { Bladder }\end{array}$ & 479 & 10.0 & $13 *$ & 460 & $600 *$ \\
\hline Lumbar Spine & 659 & 23.0 & 26 & 628 & 670 \\
\hline
\end{tabular}

*DRLs for kidney-ureter-bladder are provisional

angiography: dose-area product (DAP), $30 \mathrm{~Gy} . \mathrm{cm}^{2}$, and cumulative air kerma at the reference point, $0.5 \mathrm{~Gy}$. These DRLs were published on the ARPANSA website on 31 March 2020 [3]. The panel also made recommendations to revise the procedures included in the IGIP survey.

Conclusion Australia's DRL program is now well established. Current data for adult CT is consistent with the national DRLs. There is now sufficient data to establish a DRL for KUB scans. National DRLs for diagnostic coronary angiography have been established.

\section{References/Acknowledgements}

1. Australian Radiation Protection and Nuclear Safety Agency (2019) Code for Radiation Protection in Medical Exposure. ARPANSA. https://www.arpansa.gov.au/regulation-and-licensing/ regulatory-publications/radiation-protection-series/codes-and-standards/ rpsc-5 Accessed 31 May 2020

2. Australian Government Department of Health (2015). DIAS Practice Accreditation Standards (from 1 January 2016). Department of Health. http://www.health.gov.au/internet/main/publishing.nsf/ Content/di-DIAS-Prac-Accred-Standards-1-January-2016. Accessed 31 May 2020

3. Australian Radiation Protection and Nuclear Safety Agency (2020) Current Australian national diagnostic reference levels for image guided and interventional procedures. https://www.arpansa. gov.au/research-and-expertise/surveys/national-diagnostic-referencelevel-service/current-australian-drls/igipAccessed 31 May 2020

\section{O009 ARPANSA's new Occupational Radiation Exposure training materials}

\section{A J Mason, P D Thomas}

Medical Imaging Section, Australian Radiation Protection and Nuclear Safety Agency, Australia. (alan.mason@arpansa.gov.au [Presenting author]), (peter.thomas@arpansa.gov.au)

Introduction ARPANSA has developed and published free online Occupational Radiation Exposure material for all people working in 
medical facilities that use ionising radiation, for example those using $\mathrm{X}$-rays/CT and nuclear medicine. The modules are brief (10-35 minutes in total) filling a 'gap' identified by medical physicists, trainers, RSOs and regulators.

The ORE modules provide useful information on occupational radiation protection and safety for everyone, ranging from staff in local medical imaging facilities through to those in our major hospitals. The material is tailorable by occupation (such as nurses, cleaners, radiologists etc.) and by the level of involvement with radiation (for example, those in administration only need a limited amount of information, compared to staff in a nuclear medicine department).

Method ORE for medical facilities was developed iteratively with key stakeholder input including the professional colleges, regulators, facilities experts and end users, using modern learning strategies and techniques.

Results ORE is an innovative set of training material. Its modular structure and flexible navigation allows for the key medical professions and support functions to individually tailor the content to better suit their needs. The modular nature also makes additions and changes easy. Most facility staff will complete the module as a tailored online course via the ARPANSA website, with no login required. The training material can also be downloaded to an organisation's SCORM compliant eLearning system or as an interactive PDF/PowerPoint to facilitate group question and answer sessions.

\begin{tabular}{l}
\hline Occupational Radiation Exposure - medical facilities \\
\hline
\end{tabular}

Occupational Radiation Exposure - medical facilities
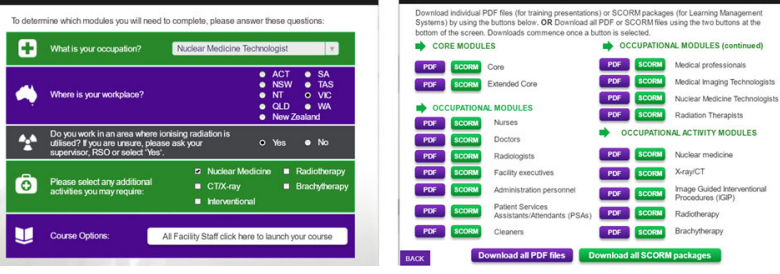

Conclusion Evidence has shown that all staff in medical facilities utilising ionising radiation can benefit from increased medical radiation safety awareness, and a common language and basis for understanding assists everyone. ARPANSA's ORE training materials fill a much needed gap. Feedback is still being sought, with the improvement process ongoing.

References/Acknowledgements Australian Radiation Protection and Nuclear Safety Agency. [Cited 29 May 2020]. Occupational Radiation Exposure for medical facilities. Available from: https://www. arpansa.gov.au/our-services/training/occupational-radiation-exposuremedical-facilities

\section{O010 TLD validation of commercial software for organ dosimetry of 1-year old infants}

\author{
M Lawson ${ }^{1,2}$, M Badawy ${ }^{1,3}$, A Kuganesan ${ }^{1}, K_{\text {Berk }^{4} \text {, }}$ \\ L McIntosh ${ }^{4}, \mathbf{Y ~ Q i}^{2}, \mathbf{P}$ Metcalfe $^{2}$
}

${ }^{1}$ Monash Imaging, Monash Health, Australia. (Ahilan.Kuganesan@monashhealth.org). ${ }^{2}$ Centre for Medical and Radiation Physics, University of Wollongong, Australia. (Michael.lawson@monashhealth.org [Presenting author]), (Yujin@uow.edu.au), (Metcalfe@uow.edu.au). ${ }^{3}$ Department of Medical Imaging and Radiation Sciences, School of Primary and Allied Health Care, Faculty of Medicine, Nursing and Health Sciences, Monash University, Clayton, VIC 3800, Australia. (Mohamed.Badawy@monashhealth.org). ${ }^{4}$ Medical Physics, Peter
MacCallum Cancer Care Centre, Australia.

(Kemal.Berk@petermac.org), (Lachlan.McIntosh@petermac.org)

Introduction Physicists and medical professionals often rely on commercial dosimetry software as a time-efficient way to estimate radiation dose for radiological examinations. In many cases, there are significant variations between the organ doses calculated by different dosimetry software, particularly in younger paediatrics. Infants are among the most radiosensitive age cohorts due to their rapidly dividing cells and longer time for possible malignant disease onset. Despite this, no studies have verified the organ doses provided by commercial software in infants undergoing computed tomography (CT) examinations. This study aims to verify the accuracy of organ doses estimated by CT Expo and NCICT for a 1-year old phantom by comparing them to thermo-luminescent dosimeter (TLD) measurements.

Method 85 high-sensitivity $\mathrm{LiF}(\mathrm{Mg}, \mathrm{Cu}, \mathrm{P})$ TLDs were embedded within a CIRS whole-body 704B one-year old anthropomorphic phantom. The phantom underwent vertex-toe CT examinations using three different CT scanners using standard hospital imaging protocols (with increased tube current). The TLDs were calibrated in dose to water using a superficial $\mathrm{x}$-ray radiotherapy unit scans. Additional CT examinations were performed with different $\mathrm{kVp}$ and added filtration. Doses were estimated using CT Expo v.2.5 and NCICT v.3.0 using the scan parameters and patient characteristics which best suited the CIRS phantom within each application, respectively.

Results The measured data indicates an over-estimation of organ doses calculated by the dosimetry software. Except for the thyroid, NCICT estimated organ doses closer to TLD measurements than CT Expo. Most organ doses estimated by NCICT were within $20 \%$ of TLD measurements across multiple kVp and CT scanners. Some CT Expo organ doses varied by more than $50 \%$ when compared to TLD measurements. Figure 1 indicate a subset of results for a single tube voltage and scanner.

Conclusion The study indicates dosimetry software tend to overestimate radiation dose in one-year olds with NCICT providing the best estimate for most organs.

\section{References}

1. Shuryak I, Sachs RK, Brenner DJ (2010) Cancer risks after radiation exposure in middle age. $\mathrm{J}$ Natl Cancer Inst 102:1628-1636

2. Brady Z, Cain TM, Johnston PN (2012) Comparison of organ dosimetry methods and effective dose calculation methods for paediatric CT. Australas Phys Eng Sci Med 35:117-134

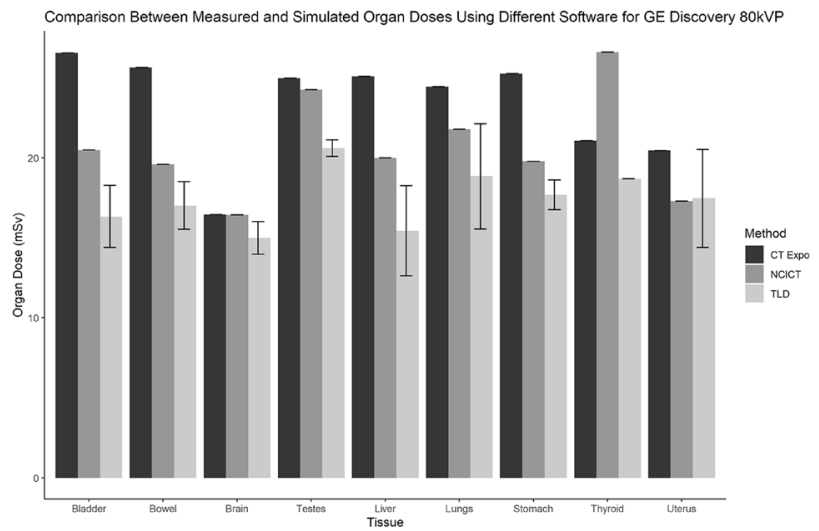

Figure 1 Comparison between TLD Measured and Software Simulated Organ Doses for GE $80 \mathrm{kVp}$ Acquisition 
Acknowledgements I would like to thank the Royal Children's Hospital in Melbourne for providing the phantom used in the study. I would also like to thank the Peter MacCallum Cancer Centre for providing the TLD dosimeters and read-out system.

\section{O011 Evaluation of scattered radiation dose to operators of hand-held dental X-ray units}

\section{J Leadbeatter, J Diffey}

Hunter New England Imaging, John Hunter Hospital, New Lambton Heights, NSW. (Justin.Leadbeatter@health.nsw.gov.au),

(Jennifer.Diffey@health.nsw.gov.au)

Introduction The portability of hand-held dental X-ray units makes them valuable for use in aged care, forensics [1], and schools. However, the close proximity of the operator to the X-ray unit raises radiation safety concerns. Tests were conducted using a Rextar $\mathrm{X}$ unit to evaluate the radiation dose to the operator during dental X-ray exposures.

Method Leakage and scattered radiation were measured with an Unfors Xi Survey detector. Scattered radiation was generated via X-ray exposures of a CTDI head phantom. Scatter dose measurements as a function of distance were made with and without a lead acrylic scatter shield $(0.6 \mathrm{mmPb}$ at $100 \mathrm{kVp})$ attached to the unit. Measurement locations were chosen to reflect the position of the operator's hands and eyes.

Results Leakage was well shielded, with a maximum of $0.02 \mathrm{uGy}$ detected at the surface of the Rextar X unit. Without the scatter shield, hand dose to the operator from a typical adult bitewing exposure was $0.69 \mathrm{uGy}$ (left) and $0.79 \mathrm{uGy}$ (right). Introducing the scatter shield reduced these doses by approximately $75 \%$, to $0.25 \mathrm{uGy}$ and $0.12 \mathrm{uGy}$ respectively. Scatter profiles obtained in the horizontal and vertical directions, with and without the scatter shield attached are shown in Figure 1.

Conclusion The Rextar $\mathrm{X}$ is well shielded, giving very little leakage radiation dose to the operator. Even without a scatter shield attached, it would take over 1000 exposures to reach $1 \mathrm{mGy}$ absorbed dose to the hands and eyes. Scattered radiation to the operator is significantly reduced when a scatter shield is attached. Although it is highly unlikely that operators would reach annual dose limits if using the Rextar X without a scatter shield, it is strongly recommended to attach one for compliance with the ALARA principle.

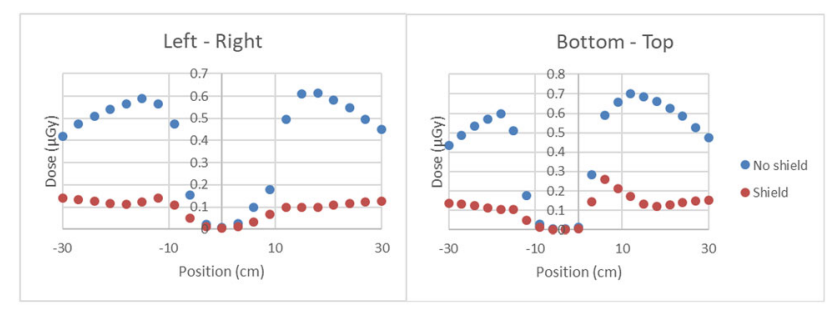

Figure 1 Scatter profiles generated by dental X-ray exposure of head phantom

\section{References/Acknowledgements}

1. Gulson AD, Holroyd JR (2016) Guidance on the Safe Use of Hand-Held Dental X-ray Equipment. Public Health England. https://www.gov.uk/government/publications/hand-held-dentalx-ray-equipment-guidance-on-safe-use. Accessed 18 May 2020

I would like to acknowledge Thomas Greig for his advice for this project. I also acknowledge Karen Sleishman and the team at Newcastle Dental Clinic for providing the Rextar X.

\section{Monte Carlo simulations of dose from intrafraction imaging in radiotherapy}

\section{McIntosh, P. Jackson, V. Peng, T. Kron, N. Hardcastle}

Department of Physical Sciences, Peter MacCallum Cancer Centre, Australia. (Lachlan.McIntosh@petermac.org [Presenting author]), (Price.jackson@ petermac.org), (Valery.peng@petermac.org), (Tomas.Kron@petermac.org), (Nick.Hardcastle@petermac.org)

Introduction Intrafraction imaging allows visualisation of tumour or surrogate during radiation therapy. As the dose from $\mathrm{kV}$ imaging is difficult to measure directly, Monte Carlo (MC) simulations can be used to obtain estimates. The imaging dose from intrafraction kilovoltage imaging was simulated for different stereotactic ablative body radiotherapy (SABR) treatments. The dose increase to target volume is presented.

Method MC software (ImpactMC) was benchmarked to match beam quality and output for the on-board imaging system of a Varian Truebeam linear accelerator. Dose/frame was calibrated by simulating a cylindrical water phantom (radius $=15.3 \mathrm{~cm}$ ), using parameters from [1]. CT scans of prostate, liver, lung, spine, and pancreas patients were used as input for simulation. Imaging system was characterised with the following parameters: $125 \mathrm{kVp}$, Ti filter (HVL $=7.98 \mathrm{~mm} \mathrm{Al}) ; 80 \mathrm{~mA}, 13 \mathrm{mS}, 7.03 \mathrm{mGy} / 100 \mathrm{mAs}$. Field size, imaging frequencies and fractionation listed in Table 1 were simulated for each anatomy, and treatment time derived from 10FFF treatments at $2400 \mathrm{MU} / \mathrm{min}$.

Results Phantom dose/frame at $15.3 \mathrm{~cm}$ depth was $0.016 \mathrm{mGy}$ compared to 0.026 in [1], differences were due to percentage-depthdose and measurement methodology. Mean dose to target for prostate, liver, lung, spine, and pancreas was 41, 162, 98, 53, and $151 \mathrm{mGy}$, respectively. This constituted $0.10,0.34,0.35,0.22$, and $0.38 \%$ of the respective total treatment dose to the target. Increased total treatment time (e.g. due to lower treatment dose rate), repeat imaging, treatment interruptions, increased dose per image frame, and peripheral location of targets resulted in a higher imaging dose.

Conclusion This study shows that dose estimates from intrafraction imaging can be estimated using MC simulation. Increased dose values were dependent on imaging isocenter location, demonstrating a relation between increased imaging dose and peripheral target volumes. In all of the treatment plans simulated, the dose increase to target volume from imaging was less than $1 \%$ (Fig. 1).

\section{References}

1. Legge, Kimberley et al. "Technical note: TROG 15.01 SPARK trial multi-institutional imaging dose measurement." Journal of Applied Clinical Medical Physics vol. 18,5 (2017) 


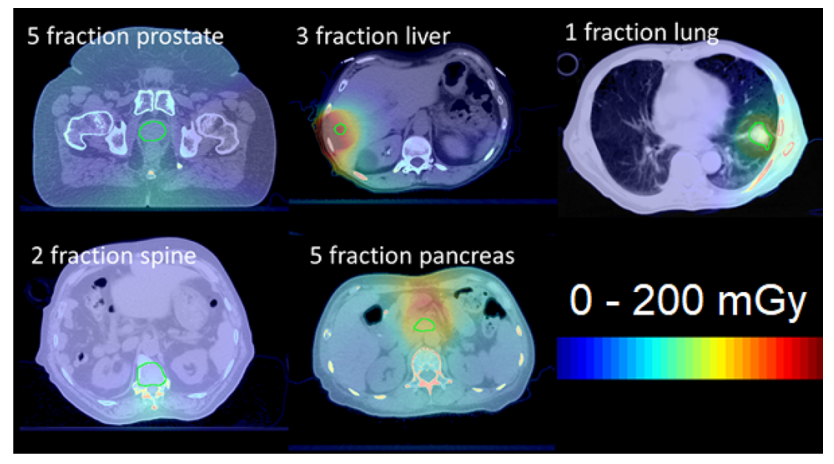

Figure 1 Dose distribution comparison for each anatomy at $11 \mathrm{~Hz}$ with a $6 \times 6 \mathrm{~cm}$ field size. Dose shown in colour scale, light green indicating target volume

Table 1 Mean dose at target for five different treatment locations

\begin{tabular}{|c|c|c|c|c|c|c|c|}
\hline \multirow[b]{2}{*}{ Region } & \multirow[b]{2}{*}{ \# Fractions } & \multirow[b]{2}{*}{ Total dose (Gy) } & \multirow[b]{2}{*}{ Field size $\left(\mathrm{cm}^{2}\right)$} & \multirow[b]{2}{*}{$\mathrm{Hz}$} & \multirow{2}{*}{$\begin{array}{l}\text { Delivery } \\
\text { Time (s) }\end{array}$} & \multicolumn{2}{|c|}{ Dose at target } \\
\hline & & & & & & Dose (mGy) & Rel. Increase \\
\hline \multirow{2}{*}{ Prostate } & \multirow{2}{*}{5} & \multirow{2}{*}{40} & \multirow{2}{*}{$6 \times 6$} & 0.2 & 100 & 0.7 & $0.00 \%$ \\
\hline & & & & 11 & 100 & 41.0 & $0.10 \%$ \\
\hline \multirow{4}{*}{ Liver } & \multirow{4}{*}{3} & \multirow{4}{*}{48} & \multirow{2}{*}{$6 \times 6$} & 0.2 & 122 & 2.9 & $0.01 \%$ \\
\hline & & & & 11 & 122 & 161.9 & $0.34 \%$ \\
\hline & & & \multirow{2}{*}{$10 \times 10$} & 0.2 & 122 & 3.4 & $0.01 \%$ \\
\hline & & & & 11 & 122 & 199.3 & $0.42 \%$ \\
\hline \multirow{4}{*}{ Lung } & \multirow{4}{*}{1} & \multirow{4}{*}{28} & \multirow{2}{*}{$6 \times 6$} & 0.2 & 222 & 1.9 & $0.01 \%$ \\
\hline & & & & 11 & 222 & 98.0 & $0.35 \%$ \\
\hline & & & \multirow{2}{*}{$10 \times 10$} & 0.2 & 222 & 2.3 & $0.01 \%$ \\
\hline & & & & 11 & 222 & 125.6 & $0.45 \%$ \\
\hline \multirow{2}{*}{ Spine } & \multirow{2}{*}{2} & \multirow{2}{*}{24} & \multirow{2}{*}{$6 \times 6$} & 0.2 & 124 & 0.9 & $0.00 \%$ \\
\hline & & & & 11 & 124 & 52.8 & $0.22 \%$ \\
\hline \multirow{2}{*}{ Pancreas } & \multirow{2}{*}{5} & \multirow{2}{*}{40} & \multirow{2}{*}{$6 \times 6$} & 0.2 & 120 & 2.7 & $0.01 \%$ \\
\hline & & & & 11 & 120 & 150.7 & $0.38 \%$ \\
\hline
\end{tabular}

\section{O013 National and international FAIR infrastructure for collaborative quantitative imaging and clinical trials research}

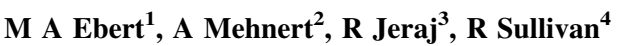 \\ ${ }^{1}$ Department of Radiation Oncology, Sir Charles Gairdner Hospital \\ and School of Physics, Mathematics and Computing, University \\ of Western Australia, Perth, Australia. \\ (Martin.Ebert@health.wa.gov.au [Presenting author]). ${ }^{2}$ Centre \\ for Microscopy, Characterisation and Analysis, University of Western \\ Australia, Perth, Australia and National Imaging Facility, Australia. \\ (Andrew.Mehnert@uwa.edu.au). ${ }^{3}$ Department of Medical Physics, \\ University of Wisconsin, Madison WI, USA. (rjeraj@wisc.edu). \\ ${ }^{4}$ Characterization, Research Technology Group, University \\ of Sydney, Sydney, Australia. (ryan.sullivan@sydney.edu.au)
}

Introduction There has been a rapid increase in attempts to extract quantitative features from medical imaging. Optimal data quality can be obtained via clinical trials, and the utility of such data is optimised when complying with FAIR principles (Findable, Accessible, Interoperable and Re-usable).

Method Efforts are underway to provide appropriate eResearch infrastructure for FAIR-aligned collaborative quantitative imaging and clinical trials research in Australasia. International facilities and efforts for imaging data archive and access were assessed. An international alliance was created to facilitate linkage of international datasets and expertise.

Results Efforts internationally have been driven by the demand for advanced computational approaches and the desires of industry to provide artificial intelligence solutions to clinical problems. Considerable investment is being made by the US National Cancer Institute into a "Research Data Commons" which will utilise Google's cloud data storage and analytics facilities. In Australia a coordinated effort (an "Australian Imaging Service") is underway, supported by the Australian Research Data Commons (ARDC), to provide a federated service for the management, curation and archiving of data, along with pipelines and machine learning tools for standard analyses on High Performance Computers and the cloud. It leverages standardised and integrated deployments of the open-source XNAT platform, ARDC-supported work on reproducibility and patient privacy, and accessibility via the Australian Access Federation. For the purpose of enabling international linkage, the Networks of Imaging Excellence (NIX) Alliance has been formed, for which legal, ethical and infrastructure requirements are being developed.

Conclusion The creation of robust infrastructure for imaging data sharing is challenging and short-term rewards are few. Having a coordinated national effort will help ease the challenge and open significant opportunities for research developments, innovation and translation.

References/Acknowledgements We are grateful for support from the ARDC, supporting institutions and Washington University School of Medicine.

\section{O014 Compliance with ICRU prescribing and reporting guidelines}

\section{A J Williams}

Wellington Blood and Cancer Centre, Wellington, NZ (andrew.j.williams@ccdhb.org.nz)

Introduction The ICRU has produced guidelines for how to report and prescribe 3DCRT and IMRT [1-[3]. In its radiotherapy clinical protocols, our centre states how it complies with these guidelines. For 3DCRT sites, we prescribe to the ICRU Reference Point, and for VMAT/IMRT sites, we aim to prescribe to the target PTV(s) $D_{50 \%}$ (we allow head and neck plans prescriptions to vary by $\pm 1.5 \%$ and prostate plans to vary by $\pm 1.0 \%$ from $D_{50 \%}$ ). An audit has been performed on our prescribing practice to verify the compliance with our stated aims.

Method Our Eclipse [4] TPS patient database was data-mined using an in-house application developed with the Eclipse Scripting API. The application extracted the plan date, site, prescribed dose, PTV IDs, $D_{50-\%}$ dose, and ICRU Reference Point dose. Data for all sites for all our clinical protocols that stated a prescription to a point or volume were analysed.

Results The results are shown below for two body sites, head-andneck VMAT treatments (Fig. 1A), and 3DCRT pelvic treatments e.g. rectum (Fig. 1B).

Conclusion VMAT - we are a) reviewing our plan optimisation process to better achieving the planning aim and b) looking at finalising prescriptions only after plan optimisation is complete. 3DCRT During the planning process we modify the plan normalisation to typically achieve a mean dose of $100 \%- \pm-1 \%$, then place the reference point on the $100 \%$ isodose. This results in plans with a large variation $( \pm-2.4 \%, 2 \%)$ in the ICRU Reference Point dose. However, it is not what we state in our clinical protocols that we do, does not follow ICRU-62 or 83 guidelines, and is not consistently applied. In order to address this situation, we will be moving as soon as is 

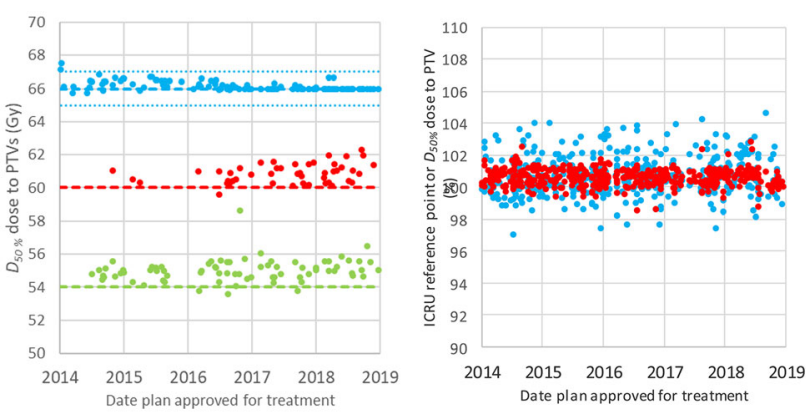

Figure 1 (A) (left) $\mathrm{D}_{50 \%}$ doses to the 66 Gy (blue), 60 Gy (red) and 54 Gy (green) PTVs for head and neck VMAT treatments. Dashed lines are the stated prescription dose levels, dotted blue lines the variation in PTV_66 $\mathrm{D}_{50 \%}$ dose allowed within our clinical protocol. Whilst the dose to the 66 Gy PTV complies with our stated aim, the doses to the other PTVs vary considerably. (B) (right) ICRU Reference Point doses (dose at the centre of the PTV) (blue) and $\mathrm{D}_{50 \%}$ doses to the target PTV (red) for 3DCRT pelvic treatments. We are not complying with our stated aim of prescribing to the ICRU Reference Point, nor prescribing to the $\mathrm{D}_{50 \%}$

practicable to prescribe all our 3DCRT treatments where a PTV is defined to the $D_{50 \%}$ dose to the PTV.

\section{References}

1. "Report 50. Prescribing, recording, and reporting photon beam therapy," International Commission on Radiation Units and Measurements, Bethesda, MD, 1993.

2. "Report 62. Prescribing, recording, and reporting photon beam therapy (supplement to ICRU Report 50)," International Commission on Radiation Units and Measurements, Bethesda, MD, 1999.

3. "Report 83. Prescribing, recording, and reporting intensitymodulated photon-beam therapy (IMRT)," International Commission on Radiation Units and Measurements, Bethesda, MD, 2008.

4. Varian Medcial Systems, Palo Alto, CA.

\section{O015 Cardiac substructure doses in a Danish radiotherapy trial cohort (DBCG HYPO): trends and modelling}

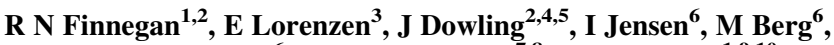
M Skovhus Thomsen ${ }^{6}$, B Vrou Offersen ${ }^{7,8}$, G P Delaney $1,9,10$, E S Koh ${ }^{1,9,10}$, D I Thwaites ${ }^{2}$, C Brink ${ }^{3,11}$, L Holloway 1,2,9,11,12

${ }^{1}$ Ingham Institute for Applied Medical Research, Liverpool, NSW. ${ }^{2}$ Institute of Medical Physics, School of Physics, University of Sydney, NSW (robert.finnegan@sydney.edu.au, Presenting author), (david.thwaites@sydney.edu.au). ${ }^{3}$ Laboratory of Radiation Physics, Department of Oncology, Odense University Hospital, Odense C, Denmark (ebbe.lorenzen@rsyd.dk). ${ }^{4}$ School of Mathematical and Physical Sciences, University of Newcastle, NSW. ${ }^{5}$ CSIRO Health and Biosecurity, The Australian e-Health and Research Centre, Herston, QLD (jason.dowling@csiro.au). ${ }^{6}$ Department of Medical Physics, Aalborg University Hospital, Aalborg, Denmark (inje@rn.dk), (martin.berg@rsyd.dk), (mette.skovhus.thomsen@aarhus.rm.dk). ${ }^{7}$ Department of Experimental Clinical Oncology, Aarhus University Hospital, Aarhus, Denmark. ${ }^{8}$ Institute of Clinical Medicine, Aarhus University,
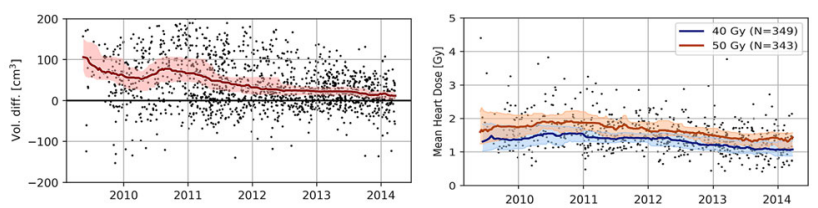

Figure 1 Trends in systematic volume differences in whole heart delineations, defined as automatic - manual (left), and mean whole heart dose (right, shown for left-sided patients receiving $40 \mathrm{~Gy} /$ hypofractionated, or $50 \mathrm{~Gy} /$ standard treatment). Thick lines/shading = 1-year rolling median/IQR

Aarhus, Denmark (Birgitte.Offersen@auh.rm.dk). ${ }^{9}$ South Western Sydney Clinical School, University of New South Wales, Sydney, NSW. ${ }^{10}$ Department of Radiation Oncology, Liverpool \& Macarthur Cancer Therapy Centres, Liverpool, NSW

(geoff.delaney@health.nsw.gov.au), (engsiew.koh@health.nsw.gov.au), (lois.holloway@health.nsw.gov.au). ${ }^{11}$ Department of Clinical Research, University of Southern Denmark, Odense, Denmark.

(carsten.brink@rsyd.dk). ${ }^{12}$ Centre for Medical Radiation Physics, University of Wollongong, Wollongong, NSW

Introduction Radiotherapy for breast cancer can increase the risks of heart disease. Patient-specific risk assessment may be improved by including the doses to cardiac substructures. Consistent delineation of these structures is critical for accurately evaluating doses.

Method Auto-contouring was used to delineate cardiac substructures in a Danish Breast Cancer Group hypofractionation trial (DBCG HYPO) dataset including over 1500 women. Trends in contouring practices and cardiac doses over time were investigated, and predictive models for substructure doses, based on doses to the whole heart, were fit to the data.

Results Manual contouring consistency improved, and the dose to the heart and cardiac substructures decreased over the study period (Figure 1). Prediction of mean substructure doses is accurate, with $\mathrm{R}^{2}$ scores in the range $0.45-0.95$ (average 0.77 ).

Conclusion Auto-contouring provides objective cardiac substructure doses. The predictive models from this work can estimate these data in situations where patient imaging and dose distributions are not available if the treatment technique is similar to that in the trial.

Acknowledgements The DBCG HYPO trial was supported by the Danish Cancer Society

\section{O016 Validation and integration of a Scale-invariant feature transform (SIFT) tool for clinical deformable image registration}

\section{Shrikant Deshpande ${ }^{1,2}$, Phillip Chlap ${ }^{3}$, Yuvnik Trada ${ }^{1,4}$, Ewa Aren $^{1}$, Lois Holloway ${ }^{1,2,4,5}$}

${ }^{1}$ Liverpool and Macarthur cancer therapy centre and Ingham Institute, Liverpool, Australia. (Ewa.Aren@ health.nsw.gov.au), (yuvnik.trada@health.nsw.gov.au),

(lois.holloway@health.nsw.gov.au). ${ }^{2}$ University of New South Wales, Sydney Australia. (shrikant.deshpande@health.nsw.gov.au [Presenting author]). ${ }^{3}$ University of New South Wales and Ingham Institute for Applied Medical Research, Australia. (phillip.chlap@unsw.edu.au). ${ }^{4}$ University of Sydney, Sydney, Australia. ${ }^{5}$ University of Wollongong, Wollongong, Australia

Introduction A recommendation from AAPM Task group 132 [1] is to have a dense set of landmarks to gain a complete understanding of deformable image registration (DIR) accuracy. However, manual 
placements of landmark points is very time consuming and not practical. Image matching by using a set of local interest points using the Scale-invariant feature transform (SIFT) tool was proposed by Lowe[2]. This tool can automatically extract dense landmark points. This work investigates a framework to integrate and validate the SIFT tool for use with a clinical system.

Method Figure 1 describes the tool's client-server architecture to compute the matching landmark points into existing clinical systems i.e. client (MIM software)

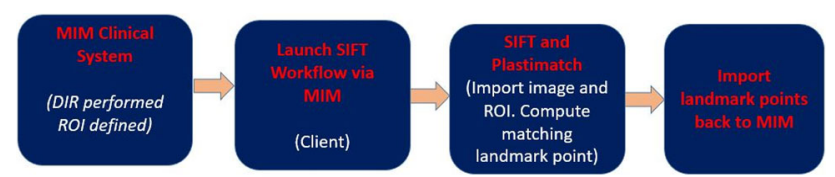

Figure 1 Client-server architecture to integrate the SIFT tool into clinical system

Either entire scan length is used or a region of interest (ROI) is created in MIM. The user runs the tool and landmark points are generated. Plastimatch [3] is used to compute matching SIFT points which are imported back into MIM. This tool was run on over 47 DIR head and neck image datasets which were acquired over different time spans and scanned in different orientations. Eight entire scan length and 44 ROIs over 40 datasets were considered. 1200 resulting landmark points were assessed to verify anatomical correspondence by a radiation oncologist.

Results An average of $12 \pm 5$ landmark points were computed within each ROI and $100 \pm 15$ over entire scan length. The majority of landmark points were detected around tissue-air or bony interfaces. Only 23 points out of the 1200 landmark points were found to be unacceptable. The time to compute the points within desired ROI takes up to 3 minutes or up to 10 minutes over the entire scan length. Conclusion The SIFT tool was successfully integrated within a clinical system and able to compute dense and reliable landmark points. The time to run this tool was found to be reasonable for clinical use.

References

1. Brock et al (2017) Use of image registration and fusion algorithms and techniques in radiotherapy: Report of the AAPM Radiation Therapy Committee Task Group No. 132 Med. Phys. 44(7):e43- e76

2. David Lowe (2004) Distinctive Image Features from ScaleInvariant Keypoints International Journal of Computer Vision $60,91-110$

3. Plastimatch (v1.8.0, www.plastimatch.org)

Acknowledgements This work supported by SWSLHD early career research grant funding.

\section{O017 Towards optimal SABR margins in prostate radiation therapy}

\section{Yutong Zhao ${ }^{1}$, Martin Ebert ${ }^{2}$, David Waterhouse ${ }^{3}$, Simon Goodall $^{3}$, Pejman Rowshan Farzad ${ }^{1}$, Shahin Fattahi ${ }^{3}$}

${ }^{1}$ Department of Physics, University of Western Australia, Perth, Australia. (Yutong.Zhao960314@gmail.com [Presenting author]), (pejman.rowshanfarzad@uwa.edu.au). ${ }^{2}$ Department of Radiation Oncology, Sir Charles Gairdner Hospital (SCGH), Perth, Australia. (Martin.Ebert@health.wa.gov.au). ${ }^{3}$ Genesis Cancer Care, Perth, Australia. (David.Waterhouse@genesiscare.com),
(Simon.Goodall@genesiscare.com),

(Shahin.Fattahi@genesiscare.com)

Introduction This research is aiming to protect bladder and rectum through giving them PRV (planning organ at risk volume) margins.

Method For each of the total of 16 patients, the bladders and rectum were delineated on CBCT images in five fractions in addition to the $\mathrm{CT}$ image set. Then the bladder and rectum wall displacement were measured through comparing CBCT and CT contours.

Based on the measured data, the relationship between the organ wall displacement frequency and percentage distance corresponding to the biggest organ wall displacement was evaluated. According to this relationship, PRV margins could be created to cover a specific percentage of organ wall motion for a specified percentage of the population.

Since the rectum wall displacement decreases from superior to inferior parts. In this research, the rectum was segmented into 3 different parts (Figure-1) based on rectum flexure and midline of the rest part of rectum in GTV. The required PRV margin for each part is suggested separately.

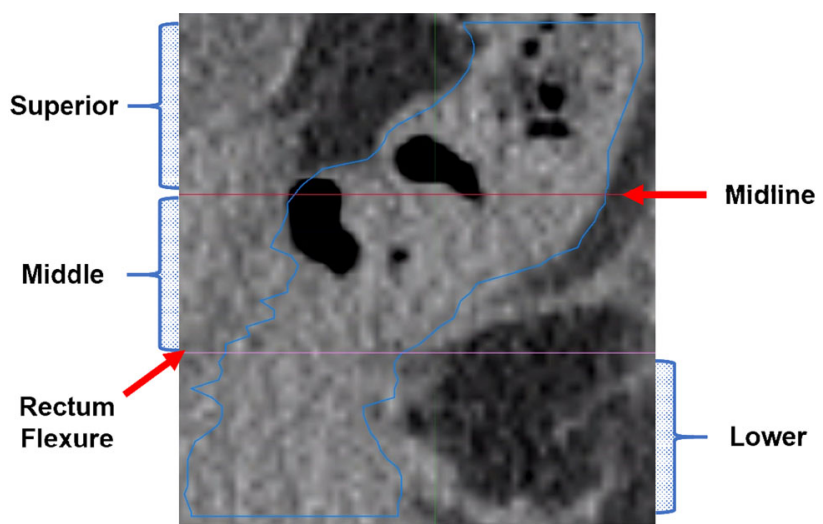

Figure 1 ACT image in the mid-sagittal plance which shows the rectum (blue contour)

Results For the bladder, a PRV margin of $0.75 \mathrm{~cm}$ (right), $0.75 \mathrm{~cm}$ (left), $1.00 \mathrm{~cm}$ (anterior), $0.80 \mathrm{~cm}$ (posterior), and $0.55 \mathrm{~cm}$ (inferior) could cover at least $90 \%$ of bladder wall outward motion (the wall motion caused by organ volume expanding) for $90 \%$ (14/15) of patients. If the effective bladder volume controlling method could be applied to control the bladder volume, a smaller bladder PRV margin with a width of $0.60 \mathrm{~cm}$ (right), $0.65 \mathrm{~cm}$ (left), $1.00 \mathrm{~cm}$ (anterior), $0.80 \mathrm{~cm}$ (posterior), and $0.45 \mathrm{~cm}$ (inferior) could be used to achieve the same goal.

For the rectum, a PRV margin as given could cover at least $90 \%$ of the rectum wall outward motion for $90 \%(12 / 13)$ of the patients.

Table 1 Derived rectum PRV margins for sections of the rectum

\begin{tabular}{|ll|l|l|}
\hline & Superior part rectum & Middle part rectum & Lower part rectum \\
\hline Right & $1.00 \mathrm{~cm}$ & $0.65 \mathrm{~cm}$ & $0.40 \mathrm{~cm}$ \\
\hline Left & $1.00 \mathrm{~cm}$ & $0.60 \mathrm{~cm}$ & $0.40 \mathrm{~cm}$ \\
\hline Anterior & $1.40 \mathrm{~cm}$ & $0.85 \mathrm{~cm}$ & $0.40 \mathrm{~cm}$ \\
Posterior & $1.00 \mathrm{~cm}$ & $1.10 \mathrm{~cm}$ & $0.55 \mathrm{~cm}$ \\
\hline
\end{tabular}

Conclusion A series of PRV margins were generated to meet the bladder and rectum motion coverage objectives. However, the clinical application of these margins needs comprehensive discussion. 


\section{O018 Can we increase PTV margins for lung cancer patients undergoing radiotherapy with deep inhalation breath hold?}

\author{
Tomas Kron ${ }^{1,2,3}$, Peta Lonski ${ }^{1}$, Nick Hardcastle ${ }^{1,3}$, Nick \\ Bucknell $^{2,4}$, Shankar Siva ${ }^{2,4}$, David Ball ${ }^{2,4}$
}

${ }^{1}$ Department of Physical Sciences, Peter MacCallum Cancer Centre, Melbourne, Australia. (Peta.Lonski@petermac.org). ${ }^{2}$ Sir Peter MacCallum Department of Oncology, University of Melbourne, Australia. ${ }^{3}$ Centre for Medical Radiation Physics, University of Wollongong, Wollongong, Australia. (Tomas.Kron@petermac.org [Presenting author]), (Nick.Hardcastle@petermac.org),

(David.Ball@ petermac.org). ${ }^{4}$ Department of Radiation Oncology, Peter MacCallum Cancer Centre, Melbourne, Australia.

(Nick.Bucknell@petermac.org), (Shankar.Siva@petermac.org).

Introduction Deep inhalation breath hold (DIBH) is a commonly used method for motion management in radiotherapy. In addition to 'arresting' the motion of the tumour it reduces lung density. We investigated how margins could change if the same mass of lung was irradiated in DIBH and free breathing (FB).

Method Lung density was assumed a surrogate for functional lung tissue and assessed using Hounsfield numbers in planning CT scans (Philips Brilliance wide bore) of thirteen breast cancer patients treated in DIBH. This was compared with density during FB. Lung density in four locations (Figure 1) was found to be on average $28+/-9 \%$ lower in the anterior parts of the lung compared to posterior ones. This resulted in more potential for lung sparing in the posterior aspects (49\% density reduction compared to $42 \%$ in the anterior parts). The average density reduction was used to calculate the increase in margin that would result in the same amount of lung in the high dose region during DIBH as in FB. Different spherical tumour volumes and planning margins were studied.

Results The additional margin facilitated by reduced density of lung during DIBH increased with increasing tumour volume, increased original margin size and the density of lung during FB (Figure 2). Margin reduction in three dimensions ranged between $1.7 \mathrm{~mm}$ for a typical lesion in stereotactic ablative radiotherapy (SABR) to $7 \mathrm{~mm}$ for a large mass with a $15 \mathrm{~mm}$ margin. In case of one-dimensional extension (eg in direction of breathing motion) the additional margin can be significantly larger.

Conclusion Assuming that lung toxicity is reduced with the density of lung in the high dose volume during radiotherapy, DIBH allows an increase of PTV margins without expected increase in toxicity, thereby potentially accounting for additional uncertainty in the reproducibility of tumour location in DIBH.

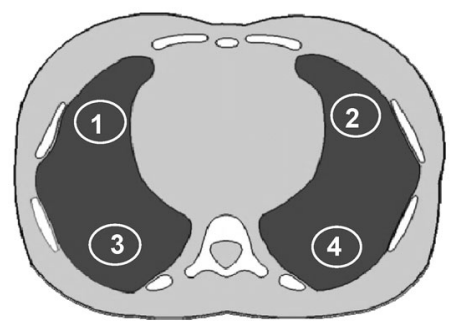

Figure 1 Locations of CT number measurements in patient. Diameter of the four regions of interest was $25 \mathrm{~mm}$

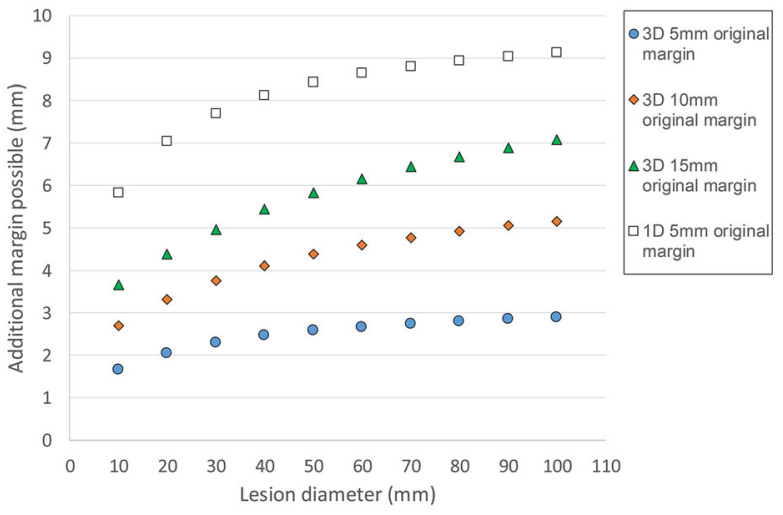

Figure 2 Additional margin possible due to reduction of lung density during DIBH. This is considering both 1 and 3 dimensional expansion of the PTV as well as different original margins for a spherical lesion of different sizes

\section{O019 Resolution of $\gamma \mathrm{H} 2 \mathrm{AX}$ in head and neck cancers following fractionated irradiation: comparison by HPV status}

Paul Reid ${ }^{1}$, Alexander H. Staudacher ${ }^{2}$, Loredana G. Marcu, Ian Olver $^{4}$, Leyla Moghaddasi ${ }^{5}$, Michael P. Brown ${ }^{6}$, Eva Bezak ${ }^{1}$

${ }^{1}$ Cancer Research Institute, University of South Australia, Adelaide, SA 5001, Australia. (Paul.reid@mymail.unisa.edu.au [Presenting author]), (Eva.Bezak@unisa.edu.au). ${ }^{2}$ Translational Oncology Laboratory, Centre for Cancer Biology, SA Pathology and University of South Australia, Adelaide, SA 5000, Australia. (alex.staudacher@health.sa.gov.au). ${ }^{3}$ Faculty of Science, University of Oradea, Oradea 410087, Romania. (loredana@marcunet.com). ${ }^{4}$ School of Psychology, University of Adelaide, Adelaide, SA 5000, Australia. (ian.olver@adelaide.edu.au). ${ }^{5}$ Genesis Care, Adelaide Radiotherapy Centre, Adelaide, SA 5000, Australia.

(Leyla.Moghaddasi@genesiscare.com). ${ }^{6}$ Cancer Clinical Trials Unit, Royal Adelaide Hospital, Adelaide, SA 5000, Australia.

(MichaelP.Brown@sa.gov.au)

Introduction Significantly better responses to therapy in head and neck cancers that result from the human papillomavirus (HPV), compared to other causes, continues to be an important focus of research activity. The positive prognostic status of HPV in head and neck cancers demonstrates a potential for dose de-escalation and a more personalised approach to therapy. A more developed understanding of treatment response, however, is required of these cancers to differentiate treatments between the 2 aetiological groups. This study is presently on-going, investigating HNSCC repair of DNA damage from fractionated X-ray dose, using measures of $\gamma \mathrm{H} 2 \mathrm{AX}$ resolution in cell lines following 4 Gy fractions and comparing between HPV positive and negative status.

Method Three HPV positive and 3 HPV negative HNSCC cell lines are grown and irradiated with 4 Gy fractions. Re-culturing of cells surviving $4 \mathrm{~Gy}$ irradiation forms the next generation of that cell line which is then irradiated with the next 4 Gy fraction. Cells of each generation of each cell line are fixed and permeabilised at 30 minutes and 24 hours following irradiation prior to staining for $\gamma \mathrm{H} 2 \mathrm{AX}$ expression and flow cytometry.

Results Comparisons of $\gamma \mathrm{H} 2 \mathrm{AX}$ recovery between 3 generations of cell line UPCI-SCC-090 show the greatest resolution in the 1st generation, from $60.4 \%$ expression at $30 \mathrm{~min}$ to $9.3 \%$ at $24 \mathrm{~h}$. The $3 \mathrm{rd}$ generation (following $3 \times 4$ Gy fractions) demonstrated the least 


\section{UPCI-SCC-090}

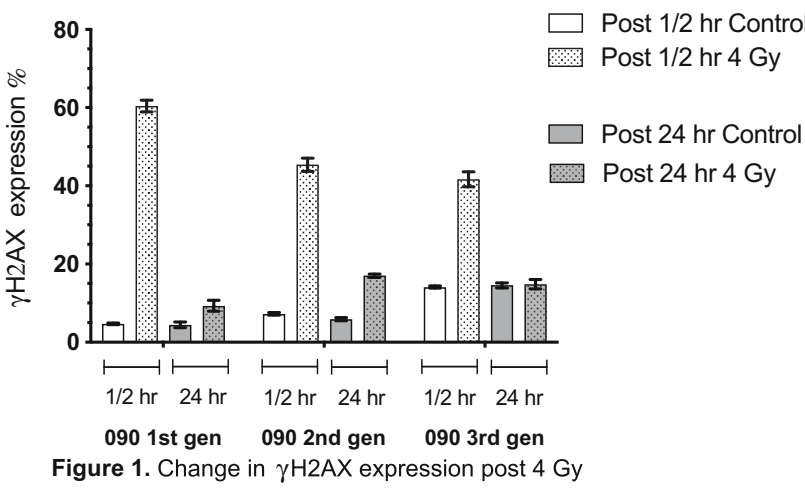

Figure 1 Change in $\gamma \mathrm{H} 2 \mathrm{AX}$ expression post $4 \mathrm{~Gy}$

recovery, $41.7 \%$ expression at $30 \mathrm{~min}$ to 14.8 at $24 \mathrm{~h}$. Background expression of $\gamma \mathrm{H} 2 \mathrm{AX}$ increased from the 1st generation level of $4.6 \%$ to $7.6 \%$ in the 2nd generation and $14.3 \%$ in the 3rd generation (Fig. 1).

Conclusion Both a diminishing capacity for cells to achieve $\gamma \mathrm{H} 2 \mathrm{AX}$ recovery and an accrual of unresolved $\gamma \mathrm{H} 2 \mathrm{AX}$ foci is observed in subsequent generations of the same cell line.

\section{O020 Auto-segmentation of the clinical target volume in a gastric cancer clinical trial}

\section{P Chlap ${ }^{1,2}$, R Finnegan ${ }^{3}$, J Dowling ${ }^{4}$, M Field ${ }^{1,2}$, K Cloak ${ }^{1,2}$,

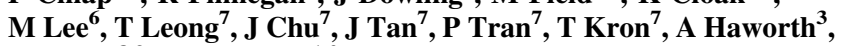 M Ebert ${ }^{8,9}$, L Holloway ${ }^{1,2}$}

${ }^{1}$ University of New South Wales and Ingham Institute for Applied Medical Research, Australia, (lois.holloway@health.nsw.gov.au).

${ }^{2}$ Department of Medical Physics, Liverpool and Macarthur Cancer Therapy Centre, Australia. (phillip.chlap@unsw.edu.au [Presenting author]), (matthew.field@unsw.edu.au), (k.cloak@unsw.edu.au). ${ }^{3}$ Institute for Medical Physics, School of Physics, The University of Sydney, Australia. (robert.finnegan@sydney.edu.au), (annette.haworth@sydney.edu.au). ${ }^{4}$ Australian e-Health Research Centre- CSIRO, Royal Brisbane Hospital, Australia. ${ }^{5}$ University of New South Wales, Australia. (jason.dowling@csiro.au). ${ }^{6}$ Liverpool and Macarthur Cancer Therapy Centre, Australia.

(mark.lee2@health.nsw.gov.au). ${ }^{7}$ Peter MacCallum Cancer Centre, Australia. (trevor.leong@petermac.org), (julie.chu@ petermac.org), (jennifer.tan@petermac.org), (phillip.tran@petermac.org), (tomas.kron@ petermac.org). ${ }^{8}$ Sir Charles Gairdner Hospital and University of Western Australia, Australia. ${ }^{9}$ School of Physics, Mathematics and Computing, University of Western Australia, Australia. (martin.ebert@uwa.edu.au).

Introduction Consistent delineation of the Clinical Target Volume (CTV) can become challenging in clinical trials due to an often-large number of centres involved. This is the case for the international TOPGEAR clinical trial [1] where patients in one treatment arm will receive radiotherapy before surgery and the protocol for delineating the CTV requires certain anatomical landmarks to be included/
Table 1 Auto-segmentation Dice Similarity Coefficient (DSC) compared to the Inter-observer variability (IOV) DSC

\begin{tabular}{llll}
\hline Case & Auto-segmentation & IOV & Difference \\
\hline 1 & 0.86 & 0.86 & 0.0 \\
2 & 0.86 & 0.88 & 0.02 \\
3 & 0.75 & 0.87 & 0.12 \\
4 & 0.84 & 0.88 & 0.04 \\
5 & 0.76 & 0.91 & 0.15 \\
6 & 0.84 & 0.87 & 0.03 \\
7 & 0.74 & 0.93 & 0.19 \\
8 & 0.80 & 0.89 & 0.09 \\
9 & 0.76 & 0.90 & 0.14 \\
10 & 0.83 & 0.88 & 0.05 \\
Mean & $0.80 \pm 0.05$ & $0.89 \pm 0.02$ & $0.08 \pm 0.06$ \\
\hline
\end{tabular}

excluded from the structure. An auto-segmentation tool could help automatically flag treatment plans where a treating centre has potentially incorrectly defined the CTV.

Method Five Radiation Oncologists provided ground truth delineations of the CTV on 10 atlas cases. A consensus workshop was held, where these five clinicians made changes to their delineations as necessary, to ensure that all contours conform to the TOPGEAR clinical trial protocol.

A multi atlas-based auto-segmentation approach [2] was used to segment the CTV structure using this atlas set. A leave-one-out analysis was performed to assess the quality of the auto-segmentation approach and the Dice Similarity Coefficient (DSC) was computed to compare the segmentation to the observer's (ground truth) delineations. The mean DSC between each observer was also computed to compare the auto-segmentation quality against inter-observer variability.

Results The mean DSC of the auto-segmentation was $0.8 \pm 0.05$ compared to $0.89 \pm 0.02$ for inter-observer variability (Table 1 ). For half of the cases the auto-segmentation DSC score was near to what was seen in inter-observer variability ( $<=0.05$ difference).

Conclusion The auto-segmentation performs well in most cases given a relatively small atlas set. The next step will be to use the probability map produced by the multi atlas-based auto-segmentation approach to quantify the uncertainty of the auto-segmentations. This tool could then help provide quality assurance for treatment plans within the TOPGEAR clinical trial as well as future gastric cancer trials.

\section{References}

1. Leong, T., Smithers, B. M., Michael, M., Gebski, V., Boussioutas, A., Miller, D., Simes, J., Zalcberg, J., Haustermans, K., Lordick, F., Schuhmacher, C., Swallow, C., Darling, G., \& Wong, R. (2015). TOPGEAR: A randomised phase III trial of perioperative ECF chemotherapy versus preoperative chemoradiation plus perioperative ECF chemotherapy for resectable gastric cancer (an international, intergroup trial of the AGITG/TROG/EORTC/NCIC CTG). BMC Cancer, 15(1). https://doi.org/10.1186/s12885-015-1529-x

2. Finnegan, R., Dowling, J., Koh, E.-S., Tang, S., Otton, J., Delaney, G., Batumalai, V., Luo, C., Atluri, P., Satchithanandha, 
A., Thwaites, D., \& Holloway, L. (2019). Feasibility of multiatlas cardiac segmentation from thoracic planning CT in a probabilistic framework. Physics in Medicine \& Biology, 64(8), 085006. https://doi.org/10.1088/1361-6560/ab0ea6

\section{O021 Deploying auto-segmentation tools in the clinic: a flexible, robust and scalable framework}

\author{
P Chlap ${ }^{1,2}$, R Finnegan ${ }^{3}$, L Holloway ${ }^{1,2}$ \\ ${ }^{1}$ University of New South Wales and Ingham Institute for Applied \\ Medical Research, Australia. ${ }^{2}$ Department of Medical Physics, \\ Liverpool and Macarthur Cancer Therapy Centre, Australia. \\ (phillip.chlap@unsw.edu.au [Presenting author]), \\ (lois.holloway@health.nsw.gov.au). ${ }^{3}$ Institute for Medical Physics, \\ School of Physics, The University of Sydney, Australia. \\ (robert.finnegan@sydney.edu.au)
}

Introduction It is often desirable to make auto-segmentation tools developed within medical physics/radiation oncology research projects available in the clinic. Ideally, these tools would integrate directly into existing workflows and clinical systems. Due to the nature of many research projects, this can often be quite challenging and require significant additional work. The framework presented in this work aims to overcome these challenges and ease the process of making auto-segmentation tools available in the clinic.

Method To ensure the framework is flexible, robust and scalable, a client-server architecture was selected (Figure 1). Like this, the client-side can prepare data and send it to the server for processing and finally download the auto-segmentations. This gives the framework the flexibility to be integrated into numerous systems already being used clinically. By implementing the auto-segmentation algorithm itself on the server-side, resources can be allocated as necessary to ensure robustness. Scalability is also achieved by decoupling the server from the client, as it can be easily replicated across multiple servers or sites.

Images are retrieved and sent using the DICOM standard. To provide more control and customization ability HTTP requests are also used for communication between the client-side and the server-side.

Results So far, a cardiac and a bronchial tree auto-segmentation algorithm have been implemented clinically at Liverpool and Macarthur Cancer Therapy Centre using this framework. An extension was made for MIM (MIM Software Inc., USA) to act as the

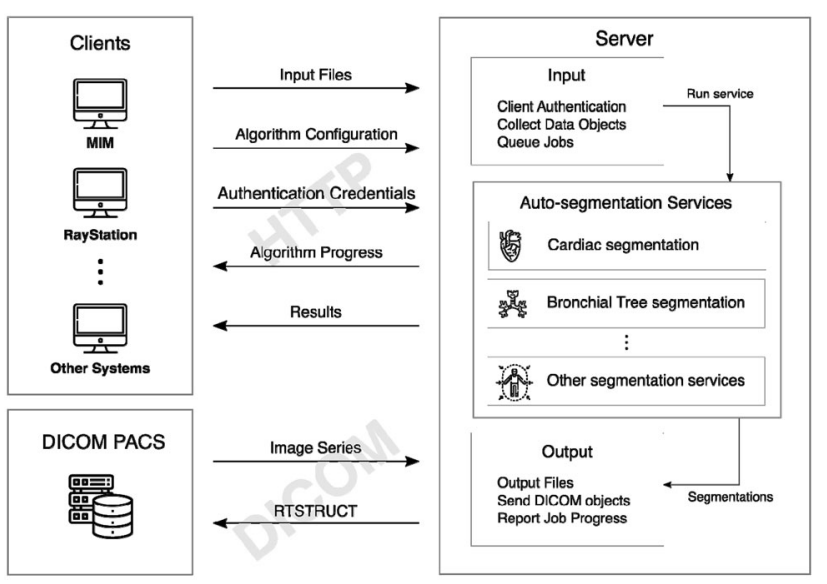

client side and a similar extension is currently being developed for RayStation (RaySearch Laboratories AB, Sweden).

Conclusion The framework presented can help overcome the challenges of implementing an auto-segmentation algorithm in the clinic. It has proven to be useful beyond deploying auto-segmentation tools in the clinic, such as in data mining research projects.

\section{O022 Alignment of computed tomography (CT) images to reference geometry by convolutional neural network}

\section{P Jackson ${ }^{1}$, J Korte ${ }^{1,2}$, L McIntosh ${ }^{1}$, T Kron ${ }^{1}$, J Ellul $^{3}, \mathrm{~J} \mathrm{Li}^{4}$, M Gaudreault ${ }^{1}$, N Hardcastle ${ }^{1}$}

${ }^{1}$ Department of Physical Sciences, Peter MacCallum Cancer Centre, Australia. (price.jackson@ petermac.org [Presenting author]), (Lachlan.McIntosh@petermac.org), (james.korte@petermac.org), (Tomas.Kron@petermac.org), (Mathieu.Gaudreault@petermac.org), (Nick.Hardcastle@petermac.org). ${ }^{2}$ Department of Biomedical Engineering, School of Engineering, University of Melbourne, Australia. ${ }^{3}$ Office of Cancer Research, Peter MacCallum Cancer Centre, Australia. (Jason.Ellul@ petermac.org). ${ }^{4}$ Bioinformatics Core Facility, Peter MacCallum Cancer Centre, Australia. (Jason.Li@petermac.org)

Introduction This work presents a methodology to efficiently associate CT slices with a standardised coordinate location. A reference geometry is defined along the patient superior-inferior axis using the typical distance between several anatomical landmark locations. An image recognition neural network was trained to associate slice appearance with a value corresponding to these reference locations. In this manner, images of an anatomical region will obtain similar coordinate values regardless of patient size or DICOM couch location.

Method One hundred CT image series were manually labelled at landmarks including femoral heads $(0 \mathrm{~mm})$, kidneys $(+246 \mathrm{~mm})$, liver dome $(+376 \mathrm{~mm})$, shoulder $(+541 \mathrm{~mm})$, and brain $(+748 \mathrm{~mm})$. Reference spatial locations were designated based on mean distance between sequential landmarks for all cases. Intermediate slice positions were interpolated linearly to efficiently generate training data for all values between vertex and thighs. Model training utilised a modified version of the Xception neural network [1] to associate image appearance with a scalar value representing reference geometry location. Model loss used the ADAM optimiser to minimise the squared distance between predicted and true slice positions over 100 epochs. Accuracy assessed by k-fold cross validation (5x 80/20 traintest split).

Results The trained location recognition network could reliably associate CT slice appearance with geometry location with an overall accuracy of $\pm 12 \mathrm{~mm}$ across all scan regions. Figure 1 illustrates the predicted $\mathrm{z}$-axis location for a typical case plotted against the original DICOM physical coordinate. Additionally, its use in identifying crop regions to assist with organ segmentation is depicted.

Conclusion This work shows the potential to use modern image recognition to evaluate $\mathrm{CT}$ scan locations and the utility of a standardised coordinate system for medical image analysis tasks. The technique may assist with longitudinal co-registration, organ localisation, and automated assessment of CT scan lengths for purposes of dose optimisation.

References/Acknowledgements As applicable.

1. Chollet, François. "Xception: Deep learning with depthwise separable convolutions." Proceedings of the IEEE conference on computer vision and pattern recognition. 2017.

Figure 1 Overview of the framework 


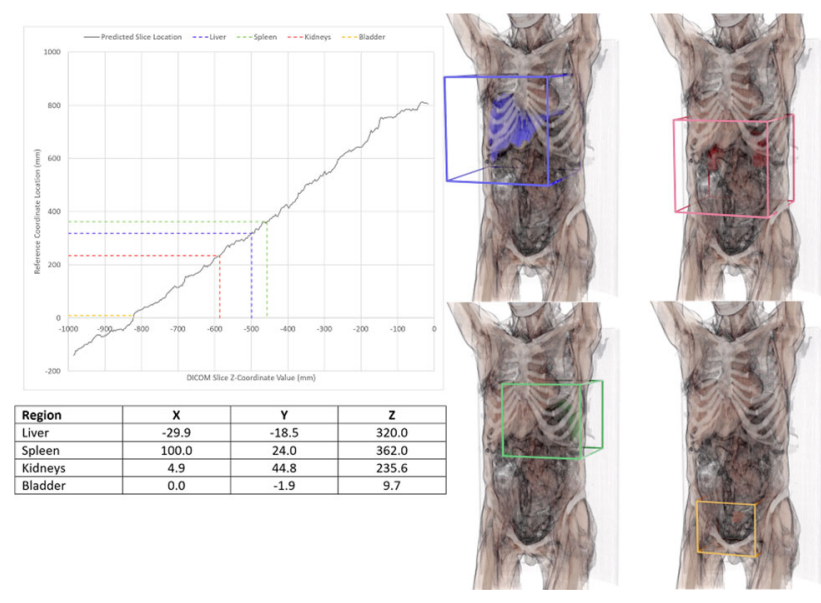

Figure 1 Application of slice recognition network to localise crop regions for subsequent image segmentation. Predicted slice locations are plotted against original (arbitrary) DICOM z-axis value. Generic crop centroids for kidneys, liver, spleen, and bladder are indicated by dashed lines and corresponding 3D box depicted on right figure set. In this example, $x \& y$ offsets are derived from physical centre-of-mass

\section{Automatic contour quality assurance based on transfer learning of a multichannel 3D ResNet}

\author{
H Min ${ }^{1,2,3}$, J Dowling ${ }^{1,3,4,5,6}$, M G Jameson ${ }^{2,3,4,7,8}$, \\ K Cloak $^{2,3}$, MJ Faustino ${ }^{7}$, M Sidhom ${ }^{7}$, J Martin', J de Leon ${ }^{10}$, \\ M Berry ${ }^{7}$, D Pryor ${ }^{11}$, P Greer ${ }^{6,9}$, LC Holloway ${ }^{2,3,4,5,7}$ \\ ${ }^{1}$ CSIRO Australian e-Health Research Centre, Herston, QLD. \\ (Hang.Min@csiro.au [Presenting author]), \\ (Jason.Dowling@csiro.au). ${ }^{2}$ Ingham Institute for Applied Medical \\ Research, Sydney. ${ }^{3}$ South Western Clinical School, University \\ of New South Wales, Australia. (k.cloak@unsw.edu.au). ${ }^{4}$ Centre \\ for Medical Radiation Physics, University of Wollongong. ${ }^{5}$ Institute \\ for Medical Physics, The University of Sydney. ${ }^{6}$ School \\ of Mathematical and Physical Sciences, University of Newcastle. \\ (peter.greer@newcastle.edu.au). ${ }^{7}$ Liverpool and Macarthur Cancer \\ therapy Centres, Australia. (Maria.Faustino@health.nsw.gov.au), \\ (Mark.Sidhom@health.nsw.gov.au), \\ (Megan.Berry@health.nsw.gov.au), \\ (Lois.Holloway@health.nsw.gov.au). ${ }^{8}$ GenesisCare, Sydney, \\ Australia (current institution). (michael.jameson@genesiscare.com). \\ ${ }^{9}$ Calvary Mater Newcastle Hospital, Radiation Oncology, Newcastle, \\ Australia. (Jarad.Martin@calvarymater.org.au). ${ }^{10}$ Illawarra Cancer \\ Care Centre, Wollongong, Australia. \\ (Jeremiah.DeLeon@health.nsw.gov.au). ${ }^{11}$ Princess Alexandra \\ Hospital, Brisbane, Australia. (David.Pryor@health.qld.gov.au)
}

Introduction Clinical trials in radiation therapy rely on high quality contours which meet the trial protocol requirements. Our team is working towards methods to automate clinical trial QA processes to ensure consistency and reduce the amount of time required for manual review. In this study, the authors present an automatic prostate manual delineation assessing scheme (Figure 1) based on transfer learning of a multichannel 3D ResNet [1] that identifies the inaccurate contours which require major correction.

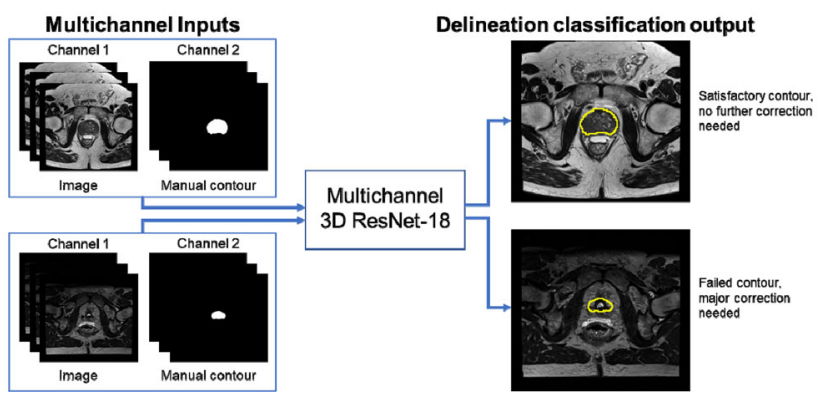

Figure 1 Diagram of the automatic delineation assessing system

Method Retrospective data from the prostate SBRT trial PROMETHEUS (ACTRN12615000223538) was utilised for this study. These data were T2-weighted magnetic resonance (MR) images including five atlas (training) cases containing gold standard prostate contours drawn by 5 expert oncologists and seventy-six trial (testing) cases containing manual contours marked as either correct or incorrect by expert observers. A multichannel 3D ResNet-18 was used to classify the manual contour as accurate or inaccurate and requiring major correction. The multichannel input consists of the MR image in the first channel and the manual contour in the second channel. Due to the limited number of training cases, the ResNet was firstly trained on another prostate MR dataset with 313 cases and then transfer learning was carried out on the five atlas training cases in our clinical radiotherapy dataset. There were no failed contours in the five goldstandard training cases, therefore, inaccurate contour samples were generated by shifting, scaling and flipping the accurate contours during training.

Results The proposed assessing system achieves a classification accuracy of 0.74 with a sensitivity for identifying incorrect contours of 0.73 and a specificity of 0.75 .

Conclusion The proposed multichannel 3D ResNet model can automatically review manual contours and identify those requiring major correction with a satisfactory sensitivity, introducing a promising approach for improving the efficiency and consistency of clinical trial quality assurance.

Acknowledgements Australian National Health and Medical Research Council (1102198), TROG, PROMETHEUS committee.

\section{References}

1. He K, Zhang X, Ren S, Sun J (2016) Deep residual learning for image recognition. In Proceedings of the IEEE conference on computer vision and pattern recognition 770-778.

\section{O024 Clinical trial contour quality assurance using transfer learning and a multichannel AtlasNet}

\author{
H Min 1,2,3, J Dowling1,3,4,5,6, MG Jameson ${ }^{2,3,4,7,8}$, K Cloak ${ }^{2,3}$, \\ M J Faustino ${ }^{7}$, M Sidhom ${ }^{7}$, J Martin', J de Leon ${ }^{10}$, M Berry ${ }^{7}$, \\ D Pryor ${ }^{11}$, P Greer ${ }^{6,9}$, L C Holloway $2,3,4,5,7$ \\ ${ }^{1}$ CSIRO Australian e-Health Research Centre, Herston, QLD. \\ (Hang.Min@csiro.au [Presenting author]), \\ (Jason.Dowling@csiro.au). ${ }^{2}$ Ingham Institute for Applied Medical \\ Research, Sydney. ${ }^{3}$ South Western Clinical School, University
}


of New South Wales, Australia. (k.cloak@unsw.edu.au). ${ }^{4}$ Centre for Medical Radiation Physics, University of Wollongong. ${ }^{5}$ Institute for Medical Physics, The University of Sydney. ${ }^{6}$ School

of Mathematical and Physical Sciences, University of Newcastle. (peter.greer@newcastle.edu.au). ${ }^{7}$ Liverpool and Macarthur Cancer therapy Centres, Australia. (Maria.Faustino@health.nsw.gov.au), (Mark.Sidhom@health.nsw.gov.au),

(Megan.Berry@health.nsw.gov.au),

(Lois.Holloway@health.nsw.gov.au). ${ }^{8}$ GenesisCare, Sydney,

Australia (current institution). (michael.jameson@genesiscare.com).

${ }^{9}$ Calvary Mater Newcastle Hospital, Radiation Oncology, Newcastle,

Australia. (Jarad.Martin@calvarymater.org.au). ${ }^{10}$ Illawarra Cancer

Care Centre, Wollongong, Australia.

(Jeremiah.DeLeon@ health.nsw.gov.au). ${ }^{11}$ Princess Alexandra

Hospital, Brisbane, Australia. (David.Pryor@health.qld.gov.au)

Introduction Manual expert review of contours for all patients enrolled in radiation therapy clinical trials prior to treatment is logistically arduous and expensive. This has led to the investigation of automated methods for improving contouring accuracy. In this study, the authors present a prostate segmentation method using multichannel AtlasNet [1] (Figure 1) which aims at generating trial atlas guided segmentations and improving manual contours which do not meet the clinical trial protocol.

Method Retrospective data from the prostate SBRT trial PROMETHEUS (ACTRN12615000223538) was utilised for this study. These data consisted of 81 T2-weighted magnetic resonance (MR) images. There were five atlas (training) cases containing gold standard prostate contours drawn by five expert radiation oncologists, and 76 trial (testing) cases containing manual contours labelled as passing or failing quality assurance. An AtlasNet architecture with multichannel input was trained to segment the prostate. The multichannel input contains the MR image in the first channel and the manual contour in the second channel. The AtlasNet consists of 4 stages: registration (rigid) to each atlas space to reduce anatomical variation; unet segmentation; inverting the rigid transform to map the segmentation back to the original image space; and segmentation fusion. Due to the limited size of the atlas training dataset, the multichannel AtlasNet was initially trained on a larger dataset with 313 cases and transfer learning was then performed on the atlas training cases.

Results The proposed system achieves an average Dice similarity coefficient (DSC) of on the whole trial set. For contours (16 contours) labelled as failing by the expert oncologists, the system improves the mean DSC between the failing and corresponding passing contour from to .

Conclusion The proposed system demonstrates accurate automatic segmentations guided by gold standard clinical trial atlases and improves failed contours of prostate.

Acknowledgements Australian National Health and Medical Research Council (1102198), TROG, PROMETHEUS committee.

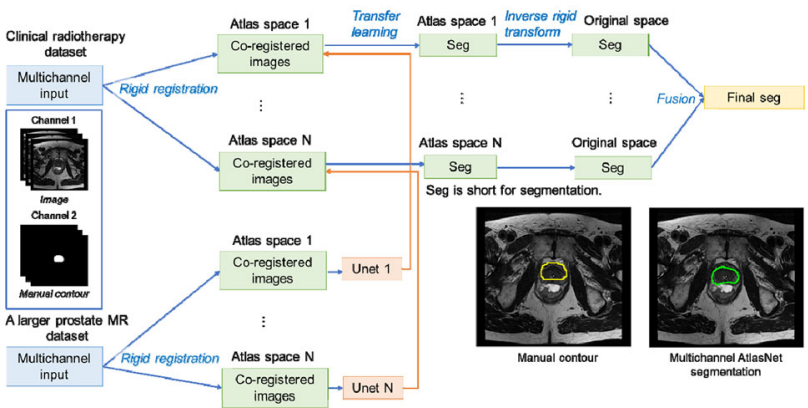

Figure 1 Diagram of the proposed system

\section{References}

1. Vakalopoulou M, Chassagnon G, Bus N, Marini R, Zacharaki E I, Revel M P, Paragios N (2018) AtlasNet: multi-atlas non-linear deep networks for medical image segmentation. In International Conference on Medical Image Computing and Computer-Assisted Intervention. 658-666. Springer, Cham

\section{O025 A probabilistic biological atlas for prostate cancer} R N Finnegan ${ }^{1}$, H M Reynolds ${ }^{2}$, Y Sun ${ }^{1}$, MA Ebert
L Holloway,
1,5,6,7, J Sykes

${ }^{1}$ Institute of Medical Physics, School of Physics, University of Sydney, NSW. (robert.finnegan@sydney.edu.au [Presenting author]), (yu.sun@sydney.edu.au),

(jonathan.sykes@health.nsw.gov.au),

(annette.haworth@sydney.edu.au). ${ }^{2}$ Auckland Bioengineering Institute, University of Auckland, NZ.

(hayley.reynolds@auckland.ac.nz). ${ }^{3}$ Department of Radiation Oncology, Sir Charles Gairdner Hospital, Nedlands, WA. ${ }^{4}$ School of Physics, University of Western Australia, Perth, WA. (martin.ebert@health.wa.gov.au). ${ }^{5}$ Ingham Institute for Applied Medical Research, Liverpool, NSW. ${ }^{6}$ Centre for Medical Radiation Physics, University of Wollongong, Wollongong, NSW. ${ }^{7}$ South Western Sydney Clinical School, University of New South Wales, Sydney, NSW. (lois.holloway@health.nsw.gov.au). ${ }^{8}$ Blacktown Cancer and Haematology Centre, Sydney West Cancer Network, Blacktown Hospital, NSW. ${ }^{9}$ School of Mathematical and Physical Sciences, University of Newcastle, NSW. ${ }^{10}$ CSIRO Health and Biosecurity, The Australian e-Health and Research Centre, Herston, QLD. (jason.dowling@csiro.au)

Introduction Radiotherapy is commonly indicated in prostate cancer treatment. Biologically based treatment planning may improve disease-free survival. This relies on accurate biological specification of the tumour. Our goal is to build a statistical biological atlas, to be used with in vivo multiparametric MRI to aid biologically based treatment planning.

Method A cohort of 70 men receiving a radical prostatectomy had in vivo and ex vivo MRI of the prostate. Tumour grade and location were marked on whole-mount histology sections. Deformable image registration (DIR) was used to combine these data in ex vivo MRI canonical space and generate a 3D distribution of tumour location and characteristics.

Results The atlas highlighted the persistence of disease in peripheralposterior prostate zones, with voxel-level probabilities for disease presence reaching maxima of $67 \%$ (Figure 1).

Conclusion Statistical atlas construction with ex vivo MRI overcomes challenges with co-registration of histology sections. A registration
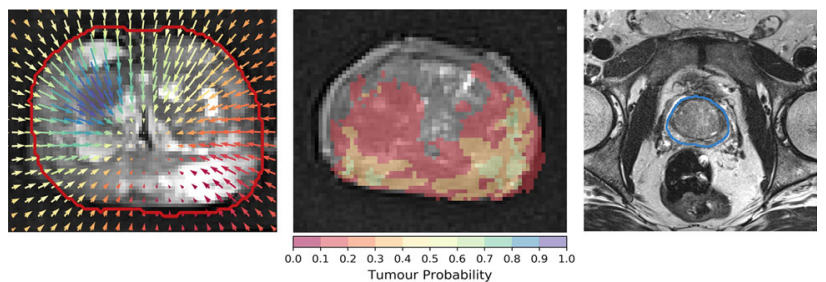

Figure 1 DIR aligns individual ex vivo MRI/histology to a canonical space (left), used to generate 3D maps of tumour location (centre). For future patients, this can refine predicted tumour distributions on MRI (right) 
framework with in vivo MRI can be used to apply this atlas to future patients for accurate biologically-optimised dose escalation.

\section{O026 Differences in sensitivity of Patient Specific Quality Assurance systems to clinically relevant treatment delivery errors - results from the SEAFARER study}

J Lehmann ${ }^{1,2,3}$, M Hussein ${ }^{4}$, S Siva $^{5}$, A Moore ${ }^{6,7}$, T Standen ${ }^{1}$, Brindha Subramanian $^{8}$, P Greer ${ }^{3,9}$, C H Clark ${ }^{4,10,11}$

${ }^{1}$ Department of Radiation Oncology, Calvary Mater Newcastle, Australia. (Joerg.Lehmann@ calvarymater.org.au [Presenting author]), (Therese.Pedersen@ calvarymater.org.au). ${ }^{2}$ Institute of Medical Physics, University of Sydney, Australia. ${ }^{3}$ School of Mathematical and Physical Sciences, University of Newcastle, Australia. ${ }^{4}$ National Physical Laboratory, Teddington, UK. (mohammad.hussein@npl.co.uk). ${ }^{5}$ Peter MacCallum Cancer Centre, Melbourne, Victoria, Australia. (Shankar.Siva@ petermac.org). ${ }^{6}$ TROG Cancer Research, Newcastle, Australia. ${ }^{7}$ University of Newcastle, Newcastle, Australia. (Alisha.Moore@trog.com.au). ${ }^{8}$ Genesis Care, Ringwood, Australia.

(Brindha.Subramanian@genesiscare.com). ${ }^{9}$ Radiation Oncology Department, Calvary Mater Newcastle, Newcastle, NSW, Australia. (Peter.Greer@newcastle.edu.au). ${ }^{10}$ University College London Hospitals, London, UK. ${ }^{11}$ National Radiotherapy Trials Quality Assurance Group, UK. (catharine.clark@nhs.net)

Introduction Patient-specific quality assurance (PSQA) requires considerable resources while its ability to detect clinically significant errors has been debated [1]. This work investigates the sensitivity of clinically used PSQA procedures to detect purposely introduced, small but clinically relevant, treatment delivery errors.

Method Seventeen facilities from Australia, the UK and the US participated in the study. Participants submitted a treatment plan file (DICOM) for a specific spine SABR treatment and received back twelve versions of that plan with small treatment delivery errors built in. These errors varied from single MLC errors, MLC bank errors, to MU and collimator rotation errors, and were the same for all facilities. Participants performed their standard clinical PSQA on each plan relative to the dose distribution of the original plan. To evaluate the impact of the errors, participants were asked to calculate und submit the dose distribution of the edited plans. Centralized DVH analysis was performed assessing D90-PTV and D003cc-SpinePRV.

Results Participants with six different planning systems were included, fifteen with Varian, two with Elekta linacs. Sixteen facilities used VMAT and one used non-coplanar IMRT. The robustness of the plans, i.e. the impact of the same errors on D90-PTV and D003ccSpinePRV, varied greatly. Of the twelve versions of a participant's plan, between two and eight passed the local clinical PSQA. Six of the error plans (from four facilities) which had caused a $>10 \%$ increase in D003cc-SpinePRV still passed PSQA. If the threshold was reduced to $>5 \%$ this rose to 18 plans (seven facilities). Consequently, multiple facilities have followed up on the findings re-assessing their PSQA process.

Conclusion There is considerable variability between different TPS and PSQA system combinations. These factors may confound the comparison of PSQA between different facilities. Care should be taken and local assessment is needed when adopting criteria and tolerances from another facility.

Acknowledgement This work has been supported by a Hunter Cancer Research Alliance (HCRA) Radiation Oncology, Cancer Imaging \& Technology (ROCIT) Flagship Program grant.

\section{References}

1. Kry SF, Molineu, Kerns $\mathbf{J}$ et al. Institutional Patient-specific IMRT QA Does Not Predict Unacceptable Plan Delivery IJROBP 90(5) 2014

\section{O027 Plan complexity metrics and their effect on detector choice for vertebral SABR patient specific quality assurance}

\author{
K Berk ${ }^{1}$, A Yeo ${ }^{1}$, T Kron ${ }^{1}$, A Montaseri ${ }^{1}$, P Krishnan ${ }^{1}$, \\ N Hardcastle ${ }^{1}$, V Hernandez ${ }^{2}$, J Saez ${ }^{2}$
}

${ }^{1}$ Peter MacCallum Cancer Centre, Victorian Comprehensive Cancer Centre, AU. (kemal.berk@ petermac.org [Presenting author]), (Adam.yeo@petermac.org), (Tomas.kron@petermac.org), (Atousa.Montaseri@petermac.org), (prem.krishnan@petermac.org), (Nick.hardcastle@petermac.org). ${ }^{2}$ Hospital Universitari Sant Joan de Reus, IISPV, Tarragona, Spain

Introduction Vertebral stereotactic ablative body radiotherapy (SABR) treatments require added precision due to close proximity of the spinal cord as a dose limiting structure. A wide range of plan complexity is observed in clinical practice. This study investigates if there is a relationship between treatment plan modulation complexity and agreement between a range of detectors and planned dose.

Method A set of 13 plans deliverable on a Trubeam STX for a wide range of complexity were obtained from a multi-institutional planning challenge. We investigated edge metric (EM) and MU/Gy. All plans were measured simultaneously using an SNC ArcCheck with a PTW microDiamond detector inserted at the target position. Three plans across the range of complexity were measured with axial Gafchromic EBT3 film. The gamma passing rate $(3 \% / 1.25 \mathrm{~mm})$ for ArcCheck and film, and point dose difference for microDiamond were assessed as a function of EM and MU/Gy.
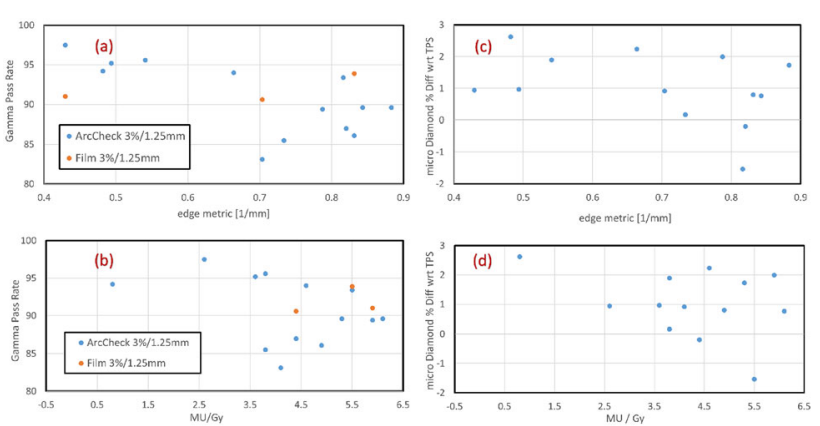

Figure 1 a Gamma pass rate vs EM for ArcCheck and Flim. b Gamma pass rate vs MU/Gy for ArcCheck and Flim. c mDiamond \%Diff vs EM. d mDiamond \% Diff vs MU/Gy

\section{Results}

There was no correlation between microDiamond and either MU/Gy (pearson $\mathrm{r}=-0.34, \mathrm{p}=0.2561$ ) or EM (pearson $\mathrm{r}=-0.39, \mathrm{p}=0.1892$ ). Figure 1 a) shows a moderate negative correlation between EM and the ArcCheck gamma results (pearson $\mathrm{r}=-0.68, \mathrm{p}=0.001$ ), but not with MU/Gy (pearson $\mathrm{r}=-0.38, \mathrm{p}=0.2056$ ) Gamma values obtained with film do not support the results obtained with the ArcCheck. As the edge metric increases, the field sizes measured by the ArcCheck 
get smaller and the limited resolution of the ArcCheck $(1 \mathrm{~cm} \times 1 \mathrm{~cm})$ fails to provide a useful value for plan assessment.

Conclusion Vertebral SABR plan dosimetric verification depends on the detector used and the modulation complexity. For film and point dose measurements, we observed no dependence on modulation complexity. The ArcCheck, however, exhibited a moderate negative correlation with edge metric, indicating limitations of the ArcCheck for complex small field dosimetry.

\section{References/Acknowledgements}

1. Maraghechi, Borna \& Davis, Jack \& Mitchell, Nicholas \& Shah, Meeral \& Fleck, Andre \& Darko, Johnson \& Osei, Ernest. (2017). The sensitivity of gamma index analysis to detect multileaf collimator (MLC) positioning errors using Varian TrueBeam EPID and ArcCHECK for patient-specific prostate volumetricmodulated arc therapy (VMAT) quality assurance. Journal of Radiotherapy in Practice. 1-12. https://doi.org/10.1017/ s1460396917000425.

\section{O028 Prediction of EPID-based VMAT patient-specific quality assurance results}

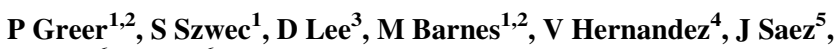

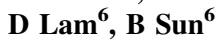

${ }^{1}$ School of Mathematical and Physical Sciences, University of Newcastle, NSW, Australia. ${ }^{2}$ Radiation Oncology Department, Calvary Mater Newcastle, Newcastle, NSW, Australia. (Peter.Greer@newcastle.edu.au (Presenting author]), (stuart.szwec@newcastle.edu.au), (michael.barnes@calvarymater.org.au). ${ }^{3}$ Allegheny Health Network, Pittsburgh, PA, USA. (danny.lee@ahn.org). ${ }^{4}$ Department of Medical Physics, Hospital Universitari Sant Joan de Reus, IISPV, Tarragona, Spain. (vhernandezmasgrau@gmail.com). ${ }^{5}$ Department of Radiation Oncology, Hospital Clínic de Barcelona, Spain.

(jordi.saez@gmail.com). ${ }^{6}$ Radiation Oncology, Washington University in St Louis, St Louis, MO, USA. (dao.lam@wustl.edu), (baozhou.sun@wustl.edu)

Introduction The aim of this work was to investigate an EPID-based patient-specific quality assurance (PSQA) procedure to determine the influence of planning parameters on the results.

Method The method uses cine images acquired in-air during VMAT to estimate $3 \mathrm{D}$ delivered dose in a virtual cylindrical phantom (VCP) to compare to the treatment planning system dose. Images were acquired using dedicated PCs with framegrabber cards and in-house software. Five linear accelerators (4 Clinacs, 1 Truebeam) and $\sim 900$ patient plans over a 6 year were analysed using batch calculations with multiple gamma criteria with a $10 \%$ of max dose threshold for inclusion. For each plan, 37 separate plan complexity metrics (PCMs) were calculated $[1,2]$. These PCMs, alongside categorical linac and planning data were used as inputs in a neural network to predict gamma-pass-rates (GPR) and mean gamma values. The importance of each PCM as well as TPS Version, linac energy, fluence mode and type were obtained from the network and the relative importance of the categorical linac and planning variables were found to be in good agreement with inferential statistical tests performed.

Results Datasets were separated into Clinac (6X) and Truebeam networks due to the different EPID/MLC, energy and fluence modes and plan types used. The models predict GPRs to within $\sim 2 \%$ at $3 \%$, $2 \mathrm{~mm}$ criteria and show high correlation with mean gamma values. The variable importance ranks were different for Clinac and Truebeam with MLC based PCMs dominating, however for Clinac model the aperture area was the most important variable, and for Truebeam
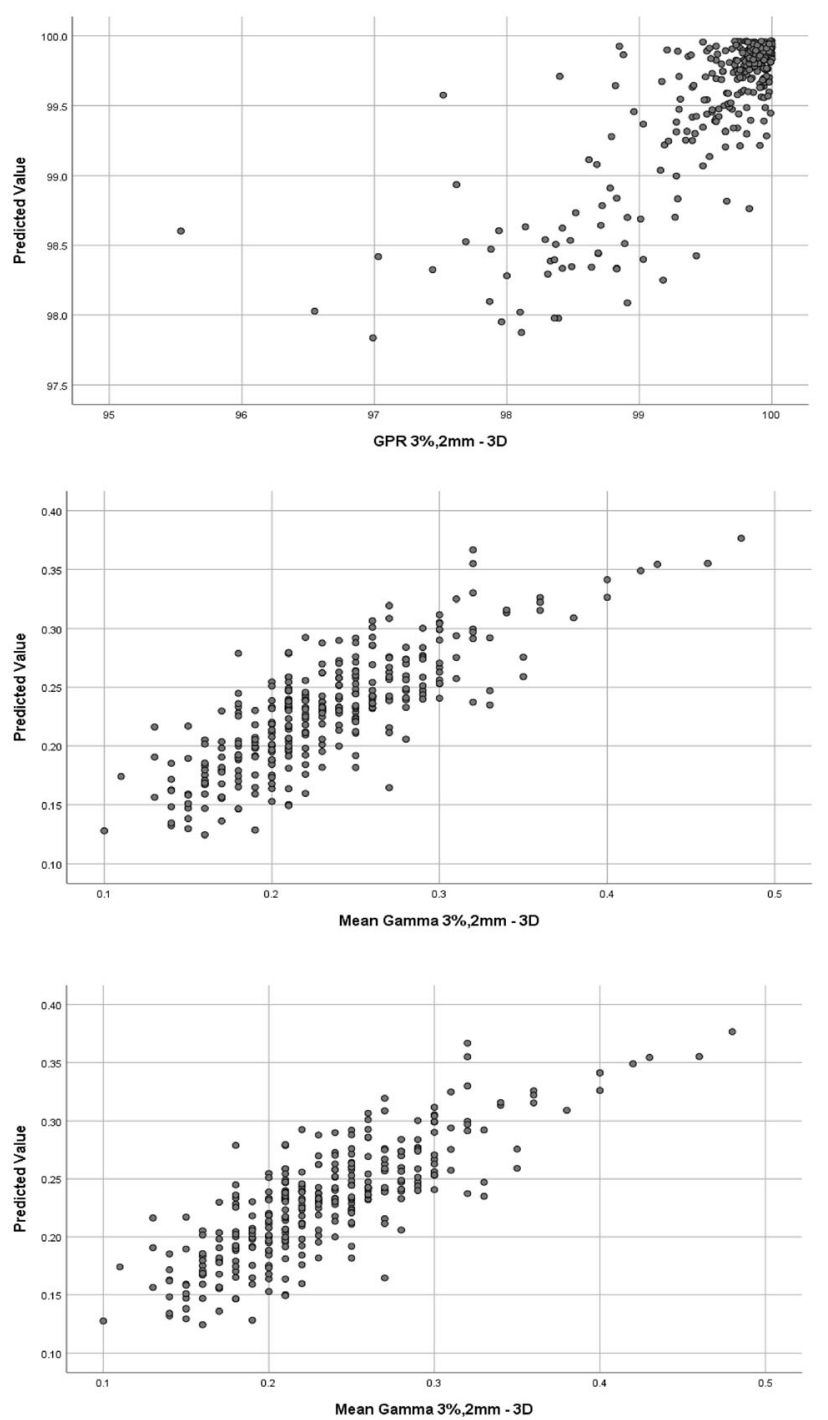

Figure 1 (upper) GPR predicted vs measured and (lower) mean gamma predicted vs measure for $3 \%, 2 \mathrm{~mm}$ gamma criteria

the aperture area weighted by MU. For Truebeam model beam energy was also an important factor.

Conclusion Aperture area was found to be the most important factor influence results of the PSQA procedure. Further investigations are required to determine if this is planning system, linac or PSQA method related (Fig. 1).

\section{References}

1. Lam D, Zhang X, Li H et al. (2019) Predicting gamma passing rates for portal dosimetry-based IMRT QA using machine learning, Med Phys 46(10):4666-4675

2. Hernandez V, Saez J, Pasler M et al. (2018) Comparison of complexity metrics for multi-institutional evaluations of treatment plans in radiotherapy, Phys Imag Rad Onc 5:37-43 
O029 Investigation of an improved method for EPIDbased IMRT and VMAT patient-specific quality assurance

\section{P Greer ${ }^{1,2}$, D Lee}

${ }^{1}$ School of Mathematical and Physical Sciences, University of Newcastle, NSW, Australia. ${ }^{2}$ Radiation Oncology Department, Calvary Mater Newcastle, Newcastle, NSW, Australia. (Peter.Greer@newcastle.edu.au [Presenting author]). ${ }^{3}$ Allegheny Health Network, Pittsburgh, PA, USA. (danny.lee@ahn.org)

Introduction The aim of this work was to develop an improved model for EPID-based pre-treatment patient-specific quality assurance (PSQA) and investigate the effect of model factors.

Method The VIPER (Virtual Phantom Epid Reconstruction) method uses images acquired in-air combined with kernels to estimate fluence and then dose in water to derive 3D combined field delivered dose distributions in virtual water-equivalent phantoms [1]. A new depthdependent dose calculation algorithm was developed that can derive dose in flat, cylindrical or spherical phantoms. Images were acquired using dedicated PCs with framegrabbers and in-house software for 5 linear accelerators (4 Varian Clinacs, 1 Truebeam) and 905 patient plans over a 6 year period. These were analysed using batch calculations with multiple 3D gamma criteria and $10 \%$ of max dose threshold for inclusion. The influence of method-specific parameters was investigated including EPID arm backscatter correction, EPID sag with gantry angle correction, angle subtended by each cine image, low-dose threshold and virtual phantom size.

Results The backscatter correction applied to Clinac images resulted in a $1.3 \%$ and $4.8 \%$ increase in mean GPR at $3 \%, 2 \mathrm{~mm}$ and $2 \%, 1$ $\mathrm{mm}$ criteria respectively. The EPID sag correction was $0.6 \%$ and $6.2 \%$. Doubling the number of frames for each image (3.2 to 6.4 degrees average angle) gave $0.2 \%$ and $1.5 \%$ decrease. GPRs decreased with increasing low dose threshold however sensitivity to PTV dose changes improved. Increasing VCP diameter from $20 \mathrm{~cm}$ to $30 \mathrm{~cm}$ improved some very large field plan results (Fig. 1).

Conclusion The new VIPER algorithm results in very high GPR for standard criteria allowing the use of more sensitive criteria. Analysis of large numbers of patient plans enables the effect of method specific parameters in the PSQA process to be understood and optimised.

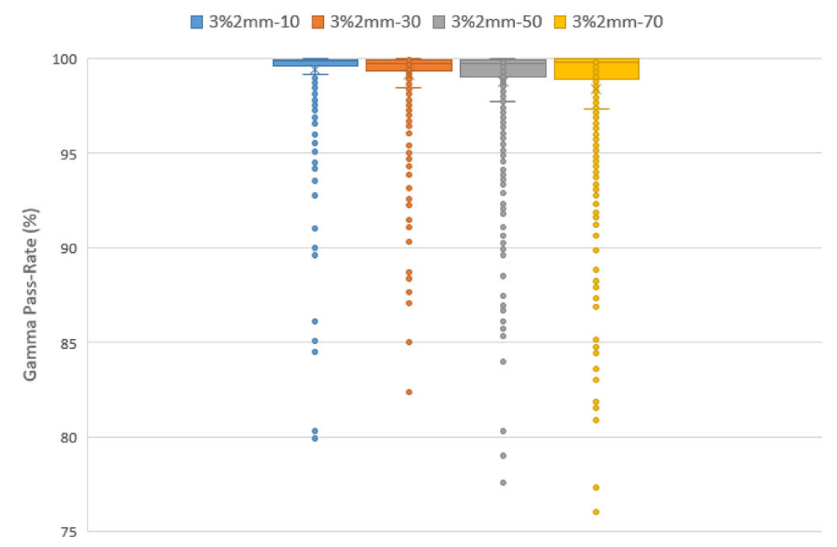

Figure 1 Effect of low dose threshold on GPR results for $3 \%, 2 \mathrm{~mm}$ criteria for 905 patient plans
Acknowledgements The authors are grateful to all staff at Calvary Mater Newcastle Hospital who collected these data.

References

1. King B, Morf D, Greer PB (2012) Development and testing of an improved dosimetry system using a backscatter shielded electronic portal imaging device. Med Phys 39(5):2839-2847

\section{O030 Validation of PerFRACTION pre-treatment QA $\left(\right.$ Fraction $0^{\mathrm{TM}}$ ) measurements against octavius measurements}

\section{S F Liu, L Webb, V Seshadri, M Wawrzak, P Hanlon, P Ramachandran}

Radiation Oncology, Princess Alexandra Hospital, Ipswich Road, Australia. (SauFan.Liu@health.qld.gov.au),

(Luke.Webb@health.qld.gov.au),

(Venkatakrishnan.Seshadri@health.qld.gov.au),

(Michal.Wawrzak@health.qld.gov.au),

(Peta.Hanlon@health.qld.gov.au),

(Prabhakar.Ramachandran@health.qld.gov.au)

Introduction Patient Specific Quality Assurance (PSQA) is mandatory for IMRT/VMAT to ensure reliable dose delivery for radiotherapy. PerFRACTION ${ }^{\mathrm{TM}}$ EPID measurement can perform pretreatment QA (Fraction $0^{\mathrm{TM}}$ ) without setting up the phantom. Fraction $0^{\mathrm{TM}} 2 \mathrm{D}$ analysis has been commissioned for IMRT/VMAT PSQA. This study compared the gamma analysis difference between PreFRACTION Fraction $0^{\mathrm{TM}}$ measurements and Octavius measurements. Method 100 previously treated clinical IMRT/VMAT plans spanning a range of treatment sites were selected for PerFRACTION measurements on different Elekta Agility beam-matched machines and validated against Octavius measurements. Dose comparison was performed with a 2D gamma analysis using the criterion available through PerFRACTION - 3\% dose difference, $2 \mathrm{~mm}$ distance to agreement, global dose normalisation, and $10 \%$ dose cut off threshold based on the AAPM TG-218 recommendation. Baseline PerFRACTION reference fields (static fields, IMRT fields, and VMAT arcs) were created for daily PA2/PA5/PA6 EPID panel constancy and calibration checks.

Results 100 IMRT/VMAT QA measurements using PerFRACTION were validated against Octavius 1500 array measurements. All 2D gamma analysis passed with both methods on two of the PerFRACTION licenced linacs PA2/PA5. The third PerFRACTION licenced linac PA6 failed 9 PSQA that was likely due to an EPID calibration issue/ panel noise. The delivery of plans to the EPID on three different linacs for analysis in PerFraction software has identified a difference in response across the EPID panels. If the PerFRACTION shows failed measurement, Octavius 1500 array 2D measurement with plastic water will be used to confirm the beams delivery.

Conclusion PerFRACTION 2D analysis of Fraction $0^{\mathrm{TM}}$ has been commissioned and is deemed safe on PA2/PA5 for clinical use. Our gamma criterion has been changed from $3 \% / 3 \mathrm{~mm}$ to $3 \% / 2 \mathrm{~mm}$ for IMRT/ VMAT PSQA using PerFRACTION 2D analysis or Octavius 1500 array 2D measurement with plastic water.

References/Acknowledgements

Miften M, Olch A, Mihailidis D, et al. Tolerance limits and methodologies for IMRT measurement-based verification QA: Recommendations of AAPM Task Group No. 218. Med Phys. 2018;45(4):e53-e83. https://doi.org/10.1002/mp.12810 


\section{O031 An evaluation of the IQM signal response and error detection sensitivity for patient specific pre- treatment QA}

\author{
T Alharthi ${ }^{1,2,3,4}$, A George ${ }^{3}$, S Arumugam ${ }^{3,5}$, L Holloway ${ }^{1,3,4,5,6}$, \\ D Thwaites ${ }^{1}$, $P$ Vial ${ }^{3,4,5}$
}

${ }^{1}$ Institute of Medical Physics, School of Physics, The University of Sydney, Sydney, New South Wales, Australia.

(david.thwaites@sydney.edu.au). ${ }^{2}$ School of Medicine, Taif

University, Taif, Saudi Arabia. ${ }^{3}$ Liverpool and Macarthur Cancer

Therapy Centers, Liverpool, NSW, Australia. ${ }^{4}$ Ingham Institute for Applied Medical Research, Sydney, NSW, Australia.

(talh5880@uni.sydneuy.edu.au [Presenting author]),

(armia.george@health.nsw.gov.au), ${ }^{5}$ South Western Sydney Clinical School, University of New South Wales, Sydney, NSW, Australia. (sankar.arumugam@health.nsw.gov.au),

(Lois.Holloway@health.nsw.gov.au),

(philip.vial@health.nsw.gov.au). ${ }^{6}$ Centre for Medical Radiation

Physics, University of Wollongong, Wollongong, NSW, Australia.

Introduction The rapid development of advanced radiotherapy techniques has increased the need for real-time patient monitoring systems $[1,2]$. An evaluation and test of the recently available Integral Quality Monitor (IQM) transmission detector is presented in this work, focussing on its ability to detect errors in photon beam delivery over a range of clinically relevant conditions.

Method The dependence of the measured signal on symmetric and asymmetric square field sizes from $1 \times 1 \mathrm{~cm}^{2}$ to $30 \times 30 \mathrm{~cm}^{2}$ was investigated. The IQM's ability to detect errors in MLC defined field size and field position was characterized. Additionally, its ability to detect delivery errors introduced to clinical VMAT plans for two different sites was assessed. This included measuring 103 nasopharynx VMAT plans and 78 lung SBRT VMAT plans with introduced variations in gantry angle, collimator angle and MLC field size and shift. Lastly, the IQM sensitivity was compared to that of the ArcCheck detector.

Results Differences between IQM calculated (expected) and measured signals remained within $\pm 2 \%$ for all symmetric field sizes used, while the asymmetric fields had significantly greater signal variation, particularly for the smallest asymmetric field sizes. The IQM showed higher sensitivity to introduced field size errors than to field shift errors for all the delivered fields and clinical VMAT plans investigated. The IQM was not able to detect collimator angle or MLC shift errors, or small gantry angle errors for the two sets of clinical plans considered. The IQM sensitivity was comparable to the ArcCheck for lung SBRT, but worse for the nasopharynx VMAT plans.

Conclusion The unique design of the IQM introduces dose response characteristics that differ from conventional dosimetry systems and care must be taken when using it for plan verification or online monitoring, especially for small fields that are off-axis in the detector gradient direction.

Acknowledgements The authors want to thank iRT for loan of the IQM transmission detector system and their support during installation and initial test of the system.

References

1. Fuangrod $\mathrm{T}$ et al (2016) Investigation of a real-time EPID-based patient dose monitoring safety system using site-specific control limits. Radiat Oncol11(1):106.

2. Thoelking J et al (2016) Patient-specific online dose verification based on transmission detector measurements. Radiother Oncol 119(2):351-356.
O032 Comparison of MLC modelling in two treatment planning systems

\author{
J Barber ${ }^{1,2}$, J R Sykes ${ }^{1,2}$, D I Thwaites ${ }^{1,2}$ \\ ${ }^{1}$ Sydney West Radiation Oncology Network, Westmead \\ and Blacktown, NSW, Australia. ${ }^{2}$ Institute of Medical Physics, \\ University of Sydney, NSW, Australia. \\ (jeffrey.barber@health.nsw.gov.au [Presenting author]), \\ (jonathan.sykes@health.nsw.gov.au), \\ (david.thwaites@sydney.edu.au)
}

Introduction Modern radiotherapy plans rely on MLC to define fields and create modulation. This work contrasts two popular TPS MLC models to determine i) the model uncertainties and ii) their impact on different classes of plans.

Method A base linac model was generated in Eclipse 13.6 and Pinnacle 9.6 for a 'golden data' Varian Clinac with Millennium MLC. A range of models were then created in both TPSs, varying leaf transmission (0,1 and 2\%) and modelling of the round leaf ends (Eclipse $\mathrm{DLG}=0,1$ and $2 \mathrm{~mm}$; Pinnacle round end offset table intercept shift by 0,1 and $2 \mathrm{~mm})$. Varied focal source models $(0,0.5$ and $1 \mathrm{~mm})$ were also investigated. Each beam model was used to calculate a series of fields, including small static and picket fence IMRT fields, and conformal and high-modulation VMAT arcs.

Results Varying focal source size gave small changes in profiles, with, Pinnacle being less sensitive than Eclipse. Larger differences were observed for leaf transmission, with varying impact across plan classes. Here, Pinnacle was more sensitive than Eclipse, especially for the highmodulation VMAT where a near-uniform dose difference was observed in- and out-of-field. Round end modelling had the largest impact on plans, with the picket fence profiles demonstrating different behaviours for abutting segments between systems (picket junction differences of $+40 \%$ and $+25 \%$ in Eclipse and Pinnacle respectively between models). High-modulation VMAT field dose changed in unpredictable ways, with local differences ranging from $-7 \%$ to $+8 \%$ and $-12 \%$ to $+7 \%$ in high and low dose regions respectively between models.

Conclusion Using this bank of fields and beam models, the significant differences in MLC modelling between systems and between plan classes is described. The modelling of MLC in the treatment planning system is of critical importance to generate accurate and deliverable plans.

\section{O033 What is wrong with Eclipse's Dosimetric Leaf Gap parameter?}

\section{F E Nelli, S Aftab}

Andre Love Cancer Centre, Geelong, Australia. (Flavio.Nelli@barwonhealth.org.au [Presenting author]), (Sadia.Aftab@barwonhealth.org.au)

Introduction Eclipse TPS uses the Dosimetric Leaf Gap (DLG) parameter for modeling the extra transmission through MLC's leaves' tips. Its experimental determination is simple and precise $[1,2]$. The TPS beam model, including DLG values, can be used to predict doses happening on experimental determination of DLG, this provides a consistency test. We found the DLG calculated after TPS predicted doses perfectly match the fed values (see Table 1). Nevertheless, several publications [3-7] point to the need of increasing DLG values to correctly predict doses in cases of highly modulated deliveries. A suboptimal tongue-and-grove modelling has been pointed as main cause of discrepancy $[3,7]$. This study further investigates the possible reasons for this contradiction. 


\begin{tabular}{|c|c|c|c|c|}
\hline \multirow{3}{*}{$\begin{array}{c}\text { Machine / } \\
\text { beam type } \\
\text { TB1 / 6X }\end{array}$} & \multicolumn{4}{|c|}{$\mathrm{DLG}[\mathrm{mm}]$} \\
\hline & \multirow{3}{*}{$\begin{array}{c}\text { Measured } \\
1.06 \\
1.06 \\
\end{array}$} & \multirow{3}{*}{$\begin{array}{c}\text { Fed into } \\
\text { Eclipse } \\
1.06\end{array}$} & \multicolumn{2}{|c|}{$\begin{array}{c}\text { Eclipse } \\
\text { predicted }\end{array}$} \\
\hline & & & AAA & 1.05 \\
\hline TB4 / 6X & & & Acuros & 1.06 \\
\hline TB1 / 10X & 1.20 & 120 & $A A A$ & 118 \\
\hline TB4 / 10X & 1.20 & $1 .<0$ & A & 1.10 \\
\hline TB1 / 10FFF & 1.11 & 111 & AAA & 1.09 \\
\hline TB4 / 10FFF & 1.11 & 1.11 & Acuros & 1.10 \\
\hline
\end{tabular}

Table 1 Measured and TPS predicted DLG for 3 photon beams delivered by 2 beam-matched TrueBeam linacs (TB1 and TB4)

Method DLG measurements were performed using a Farmer-type chamber placed on a $20 \mathrm{~cm}$ sided PMMA cube. VMAT point doses were measure with a $\mathrm{CCO} 4$ chamber inside the PMMA insert of SunNuclear's ARCCHECK devices. Doses were computed using Eclipse's AAA and Acuros algorithms (v13.6). Deliveries were carried on VARIAN's TrueBeam linacs, using 6/10 MV beams. Fluences were measured with machines' EPIs and ARCCHECK devices; analysis were performed using Eclipse's portal dosimetry algorithm (v13.6) and SunNuclear's PATIENT software (v8). We developed a theoretical framework to explain the discrepancies observed on highly modulated deliveries.

Results Experimental DLG values agree with predicted doses better than $0.5 \%$. Agreement between Eclipse predictions and measurements when using static MLC and low-modulation VMAT were found to be better than $1 \%$. However, highly-modulated deliveries showed output discrepancies as high as $\mathbf{5 \%}$, intriguingly, displaying almost identical relative dose distributions (see Figure 1). These discrepancies got reduced to less than $1.5 \%$ when DLG was increased by $0.5 \mathrm{~mm}$; with no significant on the shape of the dose distributions.

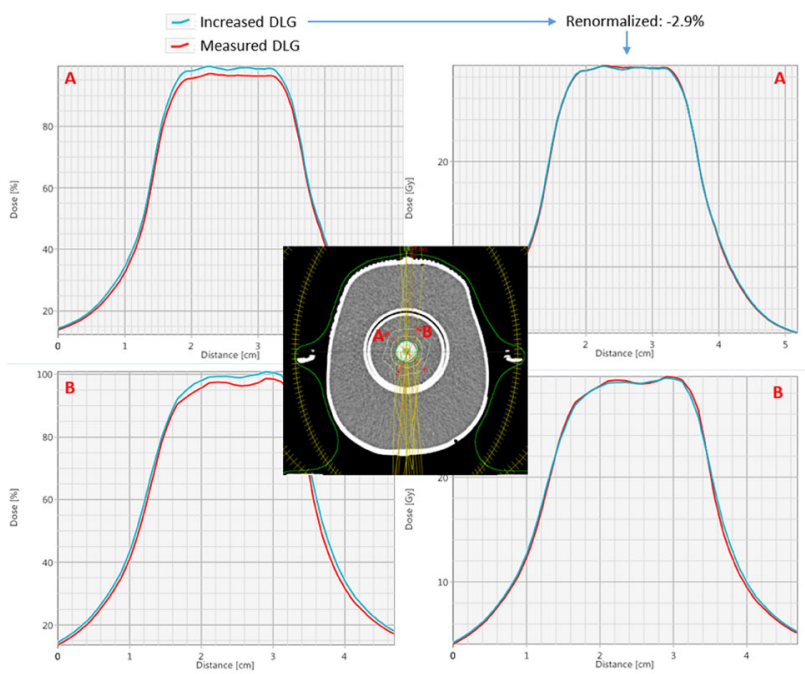

Figure 1 Eclipse predicted dose distributions, corrsponding to a brain SRS treatment type (Audit), Calculated using two different DLG values: Red distributions correspond to measured DLG, Blue distributions correspond to increased $(+0.5 \mathrm{~mm})$ DLG. Blue distributions on right side panels have been renormalized by $-2.9 \%$

Conclusion We think the need to increase DLG is rooted on two concurrent problems: The published deficiency on tongue-and-grove modelling and a deficient AAA/Acuros source model.

\section{References}

1. T. LoSasso, C.-S. Chui, and C. C. Ling: Physical and dosimetric aspects of a multileaf collimation system used in the dynamic mode for implementing intensity modulated radiotherapy. Medical Physics 25, 1919-1927 (1998).

2. M. R: Arnfield, J. V. Siebers, J. O. Kim, Q. Wu, P. J. Keal, and R. Mohan: A method for determining multileaf collimator transmission and scatter for dynamic intensity modulated radiotherapy. Medical Physics 27, 2231-2241 (2000)

3. Vieillevigne, L., Khamphan, C., Saez, J. and Hernandez, V. (2019), On the need for tuning the dosimetric leaf gap for stereotactic treatment plans in the Eclipse treatment planning system. J Appl Clin Med Phys, 20: 68-77. https://doi.org/ $10.1002 / \mathrm{acm} 2.12656$

4. Kielar, K.N., Mok, E., Hsu, A., Wang, L. and Luxton, G. (2012), Verification of dosimetric accuracy on the TrueBeam STx: Rounded leaf effect of the high definition MLC. Med. Phys., 39: 6360-6371. https://doi.org/10.1118/1.4752444

5. Middlebrook, N.D., Sutherland, B. and Kairn, T. (2017), Optimization of the dosimetric leaf gap for use in planning VMAT treatments of spine SABR cases. J Appl Clin Med Phys, 18: 133-139. https://doi.org/10.1002/acm2.12106

6. Jinkoo Kim, James S. Han, An Ting Hsia, Shidong Li, Zhigang Xu, Samuel Ryu, (2018), Relationship between dosimetric leaf gap and dose calculation errors for high definition multi-leaf collimators in radiotherapy, Physics and Imaging in Radiation Oncology, 5: 31-36, https://doi.org/10.1016/j.phro.2018.01.003.

7. Victor Hernandez et al (2017), Commissioning of the tongueand-groove modelling in treatment planning systems: from static fields to VMAT treatments, Phys. Med. Biol. 62 6688, doi.org: https://doi.org/10.1088/1361-6560/aa7b1a

\section{O034 Monte Carlo investigation on the effect of penumbra modelling on OAR dosimetry}

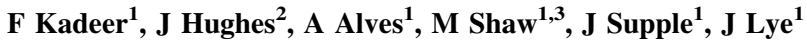 \\ ${ }^{1}$ Australian Clinical Dosimetry Service, ARPANSA, Melbourne, Australia. (fayz.kadeer@arpansa.gov.au) [Presenting author], (andrew.alves@arpansa.gov.au), (maddison.shaw@arpansa.gov.au), (jeremy.supple@arpansa.gov.au), (jessica.lye@arpansa.gov.au). ${ }^{2}$ Peter MacCallum Cancer Centre, Melbourne, Australia. (Jeremy.Hughes@petermac.org). ${ }^{3}$ RMIT University, Melbourne, Australia}

Introduction Accurate radiotherapy dosimetry relies on both dose modelling and delivery. Treatment Planning Systems (TPS) model patient dosimetry with various algorithms. Sub-optimal patient dosimetry modelling with un-optimised TPS parameters may result in underdosing target volumes, and/or overdosing organs at risk (OARs). The Australian Clinical Dosimetry Service (ACDS) identifies penumbra modelling as a potential source of discrepancy for OAR dosimetry accuracy between different TPS algorithms.

Method The ACDS audits Intensity-Modulated Radiation Therapy (IMRT) and Volumetric Modulated Arc Therapy (VMAT) across Level II (slab phantom) and Level III (anthropomorphic phantom) audits. The TG-119 "C-shape" ${ }^{\mathrm{TM}}$ is used, which is a target wrapping around a central OAR. Planned OAR dosimetry constraints ensure sufficient TPS stress-testing.

The EGSnrc Monte Carlo simulation user code models radiation transport (National Research Council Canada) ${ }^{2}$. Deliveries to a target similar in nature to the "C-shape" were simulated in water. The 


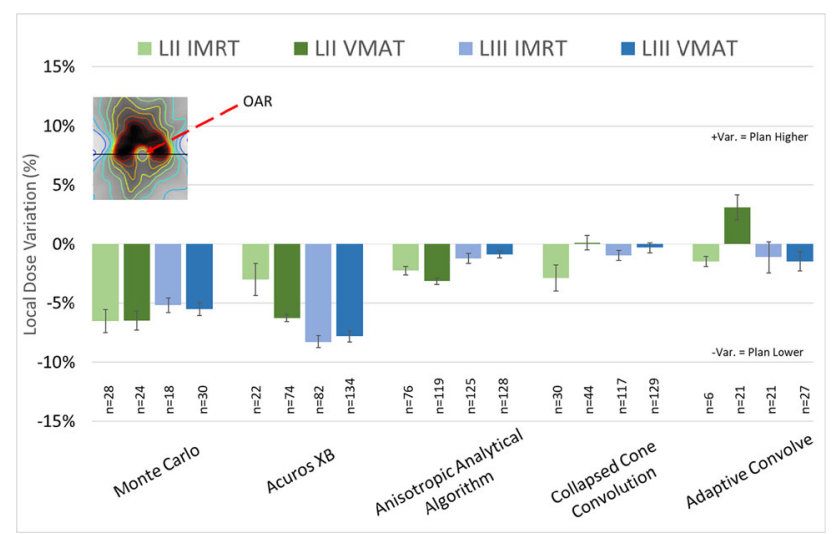

Figure 1 Local dose variations in the OAR measured by ACDS audits, with standard error bars

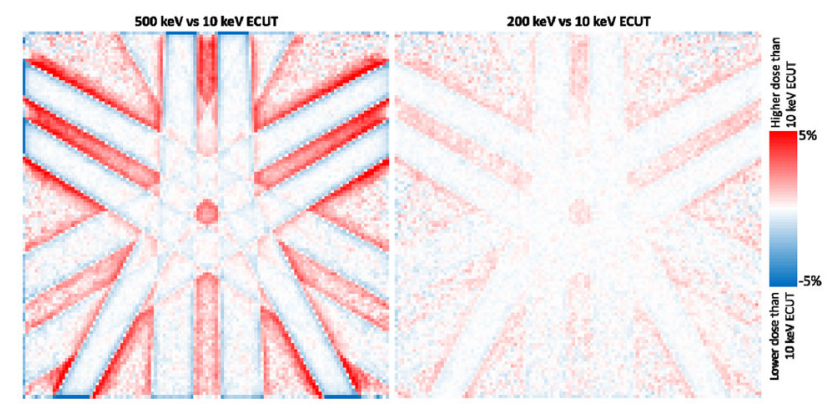

Figure 2 ECUT effect difference maps in the transverse plane. Left: $500 \mathrm{keV}$ vs $10 \mathrm{keV}$ ECUT. Right: $200 \mathrm{keV}$ vs $10 \mathrm{keV}$ ECUT

electron cut-off energy (ECUT) parameter was changed to investigate the effect on penumbra and OAR dose.

Results Separating OAR local dose variations across the ACDS national audit data by algorithm, in Figure 1, suggests variability in accuracy between algorithms at predicting OAR dosimetry.

Sub-optimal penumbra modelling is a potential source of OAR dosimetric discrepancy, and can be varied by increasing ECUT. ECUT effects to an OAR with a wrap-around target were modelled in DOSXYZnrc/BEAMnrc. The transverse plane comparisons are presented below in Figure 2.

Conclusion ACDS audit data suggests evidence for variability in the accuracy of different algorithms to predict OAR dosimetry, with the ECUT effect on penumbra modelling being identified as one potential source of this discrepancy.

Stereotactic and other small-field treatments will have larger penumbra-to-field ratios, and thus the penumbra modelling effects on OARs may be significantly more pronounced. Clinical implications may be minimal for large-field treatments, such as for prostates.

References/Acknowledgements

1. Ezzell, G.A., Burmeister, J.W., Dogan, N., LoSasso, T.J., Mechalakos, J.G., Mihailidis, D., Molineu, A., Palta, J.R., Ramsey, C.R., Salter, B.J., Shi, J., Xia, P., Yue, N.J. and Xiao, Y. (2009), IMRT commissioning: Multiple institution planning and dosimetry comparisons, a report from AAPM Task Group 119. Med. Phys., 36: 5359-5373

2. Kawrakow, E., Mainegra-Hing, D.W., Rogers, F.T., Walters, B.R., Kawrakow, I., Rogers, D., Mainegra, E., Tessier, F., Walters, B.R. (2020), The EGSnrc Code System: Monte Carlo
Simulation of Electron and Photon Transport. National Research Council Canada (NRCC).

\section{O035 Localized extra focal dose collimator angle dependence during VMAT: An out-of-field Monte Carlo study using PRIMO software}

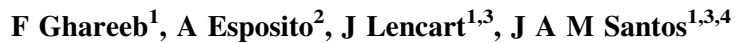 \\ ${ }^{1}$ Research Center, Portuguese Oncology Institute of Porto, Porto, \\ Portugal. (firass.ghareeb@ipoporto.min-saude.pt), \\ (joana.lencart@ipoporto.min-saude.pt), (joao.santos@ipoporto.min- \\ saude.pt). ${ }^{2}$ Radiation Oncology Princess Alexandra Raymond \\ Terrace, South Brisbane, Australia. \\ (alessandro.esposito@health.qld.gov.au [Presenting author]). \\ ${ }^{3}$ Medical Physics Department, Portuguese Oncology Institute \\ of Porto, Porto, Portugal. ${ }^{4}$ Instituto de Ciências Biomédicas Abel \\ Salazar, University of Porto, Porto, Portugal
}

Introduction Patients undergoing 3DCRT on TrueBeam LINAC equipped with HD_120_MLC are subjected to localized extra-focal dose (LEFD) in two opposed LEFD regions out of the treatment field. The dose in this region is up to $160 \%$ of mean transmitted dose through MLC [1]. VMAT implies more monitor units and irradiating more healthy tissues outside the treatment field. The aim is to investigate the dose distribution due to LEFD during VMAT and the collimator rotation angle optimization to relocate this extra dose.

Method Eight VMAT plans for a hypothetical abdominal tumor were contoured on an anthropomorphic paediatric phantom and calculated on Eclipse TPS. The plans were optimized to the same dosimetric objectives at PTV and Organ at Risk (OAR) with different collimator rotation angles. They were also simulated using PRIMO Monte Carlo software. The dose out of the treatment field was evaluated in eight

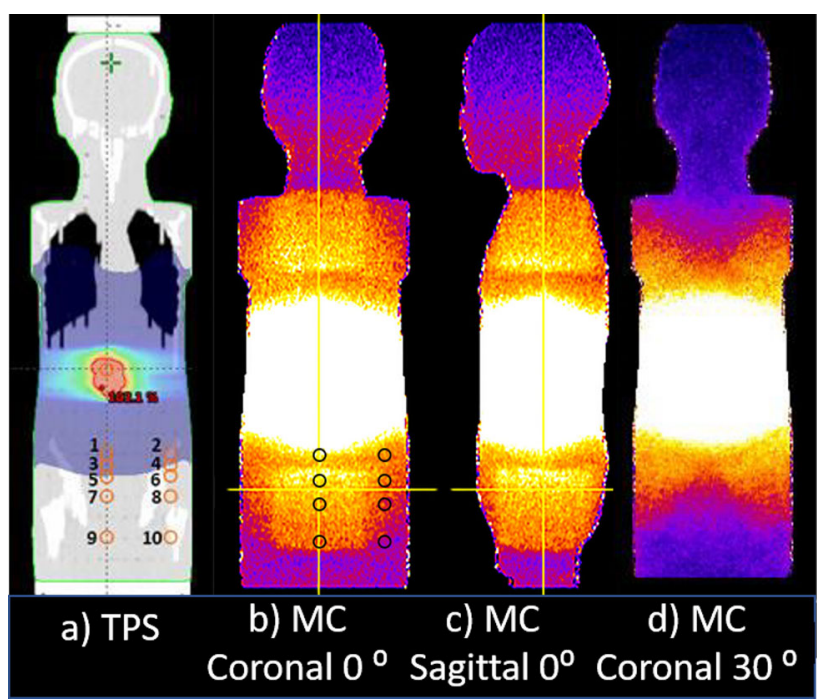

Figure 1 a) TPS calculated dose for collimation angle $0^{\circ}$ in coronal view. The sampling spheres are shown and numbered 1 to $10 . \mathrm{b}+\mathrm{c}$ ) The Monte Carlo results for the same configuration on a saturated colour scale in Coronal and Sagittal view respectively. The LEFD regions superimposed are visible. In b) the spheres are the same as in a) with the exception of spheres 3 and 4 that were removed for simplicity d) The Monte Carlo results in Coronal view when the collimator is rotated by $30^{\circ}$. The LEFD regions drifted apart 


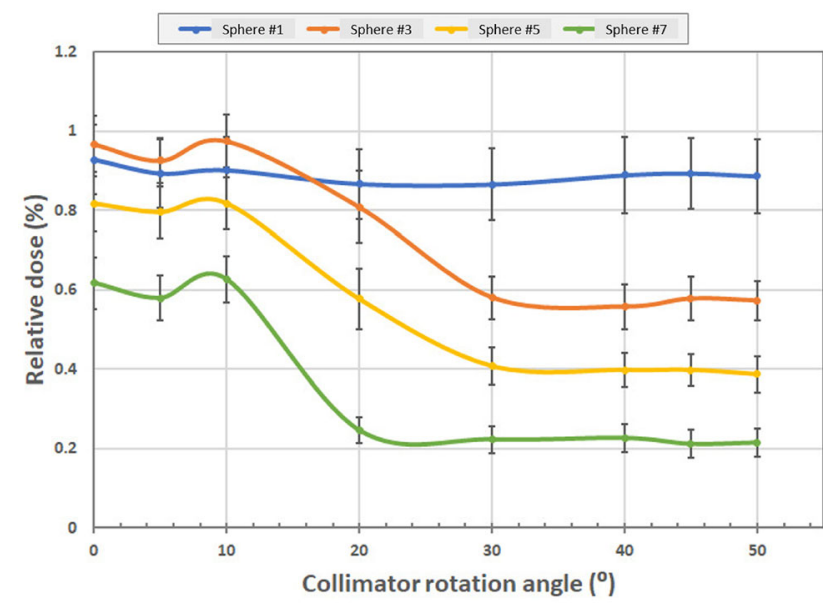

Figure 2 The relationship between the collimator rotation angles and the mean deposited dose in spheres \#1, \#3, \#5 and \#7 located along the gantry rotation axis, relative to the mean dose at the PTV. The error bars represent \pm 1 standard deviation of percent voxel values inside the spheres

spheres at different distances from the isocenter. For validation purpose, QA plans were created, measured by EPID and simulated in a virtual phantom of the EPID. Measured and simulated QA plans were compared using gamma index methodology. Point dose measurements were also used to verify Monte Carlo simulations accuracy out of the treatment field.

Results Measured and simulated QA dose distributions showed two LEFD regions per arc out of the treatment field which were not calculated by TPS. The simulated plans showed LEFD as cylindrical dose distributions, $10 \mathrm{~cm}$ height, with the diameter of $\mathrm{X}$-jaws aperture. At $0^{\circ}$ rotation the LEFD regions of the two-treatment arcs are superimposed. As rotation angle increases the two cylindrical distributions drift apart but their intersection remains localized along the central axis until they are completely separated.

Conclusion The LEFD can be avoided during VMAT without compromising the plan objectives by increasing the collimator rotation angle to relocate LEFD out of the body (Figs. 1, 2).

\section{References/Acknowledgements}

1. F. Ghareeb, J. Lencart, J. Oliveira, J.A.M. Santos, Characterization of extrafocal dose influence on the out-of-field dose distribution by Monte Carlo simulations and dose measurements, Health Phys., 117 (2019), pp. 489-503

\section{O036 Radiation dose perturbation at the tissue interface of PEEK and Titanium Bone implants: Monte Carlo simulation, film dosimetry and treatment planning}

\section{Georgio Andrew Katsifis ${ }^{1,2}$, David R. McKenzie ${ }^{1,2}$, Michael O'Connor $^{3}$, Robin $\mathrm{Hill}^{3}$, Natalka Suchowerska ${ }^{1,2}$}

${ }^{1}$ University of Sydney, School of Physics, Australia. (gkat2146@uni.sydney.edu.at [Presenting Author]). ${ }^{2}$ VectorLAB, Chris O'Brien Lifehouse, Australia. ${ }^{3}$ Department of Radiation Oncology, Chris O'Brien Lifehouse

Introduction Titanium implants cause significant dosimetric perturbations in radiotherapy. Excessive dose at the tissue-implant interface compromises osseointegration, whilst dose shielding may compromise treatment. An alternative material is the PAEK polymer family, but it requires detailed dosimetry characterisation at the interface. Here we use Monte Carlo (MC) methods to characterise the dosimetry at the tissue-implant interface of titanium and PEEK in comparison with bone.

Method The Monte Carlo simulation is designed to score the absorbed dose in $2 \mathrm{~mm}$ slices for the whole phantom and $0.2 \mathrm{~mm}$ slices near the implant to generate a depth-dose curve (Figure 1). Radiochromic film was used to measure the dose at the water-implant interface. The dose was also calculated with the Varian Eclipse TPSv15.6.

Results The Monte Carlo calculated dose perturbation is shown in Figure 2. At the incident interface, a $34 \%$ dose enhancement is observed for titanium, $12 \%$ for bone and $6 \%$ for PEEK. At the exit interface, an $18 \%$ dose decrease is observed for titanium, but a dose enhancement of $7 \%$ for PEEK and $3 \%$ for bone. There is a dose reduction of $15 \%$ for titanium and $3 \%$ for bone $5 \mathrm{~cm}$ beyond the exit interface, but enhancement of $0.3 \%$ for PEEK.

Conclusion Our results show that in a radiation beam, PEEK behaves more like bone than titanium does. By avoiding an over-response at the entrance interface $(\sim 30 \%)$, osteo-integration is not compromised, while the absence of a cold spot $(\sim 18 \%)$ at the exit interface avoids treatment failure. Film dosimetry confirms interface dose perturbation, while treatment planning underrepresents the potential impact to patients.

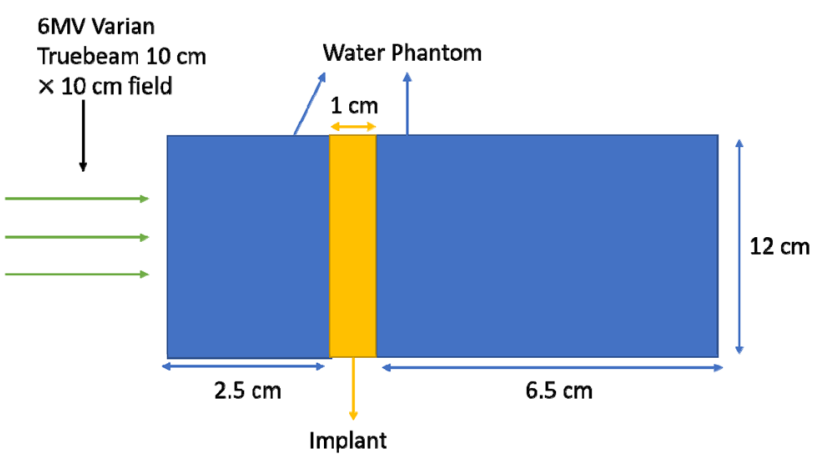

Figure 1 Study design. The Monte Carlo simulation was performed using Geant4 10.4.2 on Gate v8.1.p01

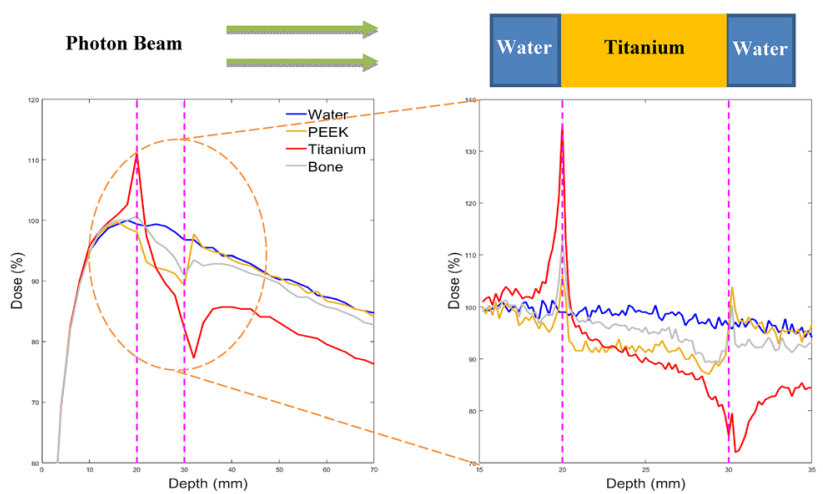

Figure 2 Monte Carlo simulated percentage depth dose curve illustrating the dose perturbation at the water-implant interface for four materials: water, PEEK, Titanium and bone. The left figure shows the dose calculated with a resolution of $2 \mathrm{~mm}$ and on the right at a higher resolution of $0.2 \mathrm{~mm}$ 
Funding Australian Government through Sarcoma Research Centre at Chris O’Brien Lifehouse.

\section{O037 Reducing sensitivity to asymmetric scattering conditions in source-tracking based treatment verification of HDR brachytherapy}

\author{
R D Franich ${ }^{1}$, J Wallace ${ }^{1}$, N Foroughimehr ${ }^{1}$, M Hanlon, V \\ Panettieri $^{2}$, J L Millar ${ }^{1,2}$, R L Smith ${ }^{2}$ \\ ${ }^{1}$ School of Science, RMIT University, Australia. \\ (rick.franich@rmit.edu.au [Presenting author]), \\ (jordan.wallace@rmit.edu.au), (foroughimehr.negin@gmail.com), \\ (maximilian.hanlon@rmit.edu.au), (Jeremy.Millar@monash.edu). \\ ${ }^{2}$ Alfred Health Radiation Oncology, The Alfred, Melbourne, \\ Australia. (v.panettieri@alfred.org.au), (Ryan.Smith@alfred.org.au)
}

Introduction Our treatment verification system [1] utilises a flat panel detector (FPD) mounted in the treatment couch to track source positions by capturing source radiation exiting the patient. Previously reported in vivo tracking capabilities have exhibited measurement uncertainties due to asymmetric scattering in patient geometries [2]. Here, we show that asymmetric scattering affects the radiation distribution at the FPD and hence the source position measurement. We demonstrate two methods to compensate for the asymmetric scattering conditions.

Method Source tracking in a phantom was conducted in symmetric and asymmetric scattering conditions, by varying proximity to air interfaces, compared to ground-truth derived from subtraction radiography [1]. Inaccuracies caused by asymmetry were mitigated using two techniques: physical by introducing an anti-scatter grid to improve the primary-scatter-ratio; and computational by adopting a data-driven optimisation of our algorithms that extract the source position from the FPD dose distribution.

Results Source position accuracy decreases from $<1 \mathrm{~mm}$ under symmetric conditions to an error of up to $9 \mathrm{~mm}$ in the most asymmetric case, thus confirming the influence of asymmetric scattering. Optimising tuneable parameters of the position determining functions reduced their sensitivity to asymmetry, with differences improving from $<9 \mathrm{~mm}$ to $<2.5 \mathrm{~mm}$. Employing the anti-scatter grid to improve the primary-scatter ratio, discrepancies improved from $<9 \mathrm{~mm}$ to $<4$ mm (see Fig. 1).

Conclusion The effect of asymmetric scattering on measured dwell positions was quantified, with errors up to $9 \mathrm{~mm}$ shown in extreme

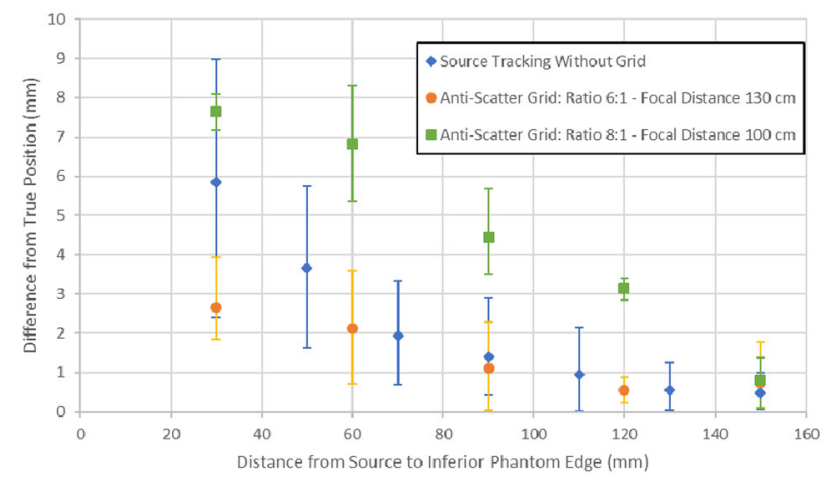

Figure 1 Difference between measured and true positions relative to the inferior phantom-air interface. Two grid results are shown against no-grid data emphasizing that grid choice is important. Error bars represent Min-Max range over 6 measurements examples. Two new approaches of reducing measurement errors due to asymmetric scatter have been demonstrated. Both physical and computational strategies were shown to improve accuracy in sourcetracking based treatment verification.

References

1. Smith, R. L., Haworth, A., Panettieri, V., Millar, J. L., \& Franich, R. D. (2016). A method for verification of treatment delivery in HDR prostate brachytherapy using a flat panel detector for both imaging and source tracking. Medical physics, 43(5), 2435-2442. http://dx.doi.org/10.1118/1.4946820

2. Smith, R. L., Hanlon, Panettieri, V., Millar, J., M., Matheson, B., Haworth, A., \& Franich, R. (2018). An integrated system for clinical treatment verification of HDR prostate brachytherapy combining source tracking with pretreatment imaging. Brachytherapy, 17, 111-121. http://dx.doi.org/10.1016/ j.brachy.2017.08.004

\section{O038 The MArkerless Lung Target Tracking CHallenge (MATCH): an AAPM grand challenge}

Marco Mueller', Per Poulsen ${ }^{2}$, Wilko Verbakel $^{3}$, Ross Berbeco ${ }^{4}$, Dianne Ferguson ${ }^{4}$, Lei Wang ${ }^{5}$, Lei Ren ${ }^{6}$, Shinichiro Mori ${ }^{7}$, John Roeske $^{8}$, Perry Zhang ${ }^{9}$, Paul Keall ${ }^{1}$

${ }^{1}$ ACRF Image X Institute, The University of Sydney, Australia. (marco.mueller@sydney.com.au [Presenting author]), (Paul.keall@sydney.edu.au). ${ }^{2}$ Aarhus University, Denmark. (per.poulsen@rm.dk). ${ }^{3}$ VU University Medical Centre, The Netherlands. (w.verbakel@amsterdamumc.nl). ${ }^{4}$ Harvard University, USA. (ross_berbeco@dfci.harvard.edu). Harvard University, USA. (Dianne_Ferguson@dfci.harvard.edu).Stanford University, USA. (leiwang@stanford.edu). Duke University, USA. (lei.ren@duke.edu). ${ }^{7}$ National Institute of Radiological Science, Japan. (mori.shinichiro@qst.go.jp). ${ }^{8}$ Loyola University Medical Center, USA. (JROESKE@lumc.edu). ${ }^{9}$ Memorial Sloan Kettering Cancer Center, USA. (zhangp@mskcc.org)

Introduction Avoiding the risks and costs involved with the surgical insertion of radio-opaque fiducials, several commercial and academic markerless lung target tracking approaches have been developed to further improve patient safety during lung cancer stereotactic ablative body radiotherapy (SABR). However, these approaches had yet to be benchmarked using a common measurement methodology. This knowledge gap motivated the Markerless Lung Target Tracking Challenge (MATCH) [1].

Method The MATCH is an American-Association-of-Physicists-inMedicine-(AAPM)-sponsored Grand-Challenge in 2019/2020. The

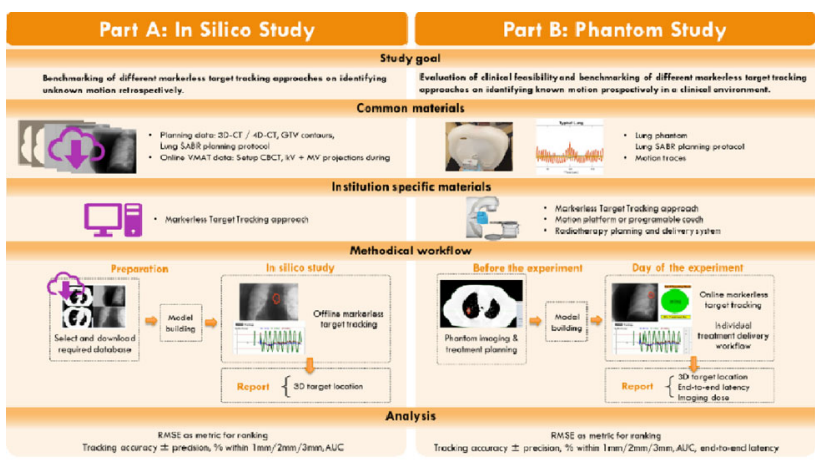

Figure 1 Overview of the AAPM MATCH Grand Challenge 
participants aim to accurately and precisely localise lung targets with time in (Part-A) a retrospective in-silico study and (Part-B) prospective phantom experiments. Common to both parts are a 3Dprinted lung phantom including three lung targets [2], and a lung SABR planning protocol [3]. The phantom is moved rigidly with patient-measured lung target motion-traces, which also act as groundtruth. In Part-A a volumetric-modulated-arc-therapy-treatment is delivered to the phantom programmed with four unknown motiontraces. A dataset consisting of treatment-planning data and intratreatment $\mathrm{kV}$ - and MV-images is provided to the participants. In Part$\mathrm{B}$ the participants use their own approach and workflow to localise the target during the dose delivery for five motion-traces. All participant submissions will be analysed and ranked based on the percentage of the tracking error values within $2 \mathrm{~mm}$ of the ground-truth. The challenge is open to any participant, and participants could complete either one or both parts (Fig. 1).

Results More than 20 institutions registered for MATCH. At the time of the abstract submission, eight results from five institutions in three countries have been received. The results of the challenge will be made available after the deadline 31st of August 2020 and presented at the EPSM conference 2020.

Conclusion A common methodology for measuring the accuracy of markerless lung target tracking algorithms has been developed and used to benchmark academic and commercial approaches retrospectively and prospectively.

Acknowledgements We thank the AAPM, Scandidos and Varian for their support.

\section{References}

1. https://www.aapm.org/GrandChallenge/MATCH/

2. Hazelaar C, van Eijnatten M, Dahele M, et al. Using 3D printing techniques to create an anthropomorphic thorax phantom for medical imaging purposes. Med Phys. 2018;45(1):92-100.

3. Videtic, Gregory MM, et al. "A randomized phase 2 study comparing 2 stereotactic body radiation therapy schedules for medically inoperable patients with stage I peripheral non-small cell lung cancer: NRG Oncology RTOG 0915 (NCCTG N0927)." International Journal of Radiation Oncology* Biology* Physics 93.4 (2015): 757-764.

\section{O039 Automatic detection and tracking of marker seeds implanted in Prostate cancer patients using a Deep Learning Algorithm}

\author{
Keya Amarsee ${ }^{1}$, Dr. Prabhakar Ramachandran², Dr. Andrew \\ Fielding ${ }^{3}$, Dr. Daryl Ning ${ }^{4}$ \\ ${ }^{1}$ Master of Applied Science (Medical Physics), Queensland \\ University of Technology. (keya.amarsee@connect.qut.edu.au \\ [Presenting author]). ${ }^{2}$ Director of Therapeutic Physics, Princess \\ Alexandra Hospital. (Prabhakar.Ramachandran@health.qld.gov.au). \\ ${ }^{3}$ Senior Lecturer \& Course coordinator, Master of Applied Science \\ and Graduate Diploma, Queensland University of Technology. \\ (a.fielding@qut.edu.au). ${ }^{4}$ Principal Applications Engineer, The \\ MathWorks. (ningd@mathworks.com)
}

Introduction Fiducial marker seeds are often used as a surrogate to identify and track the positioning of prostate volume in the treatment of Prostate cancer. This research is undertaken to develop and validate a real time deep learning object detector using a YOLO v2 Convolution Neural Network (CNN) that can detect and track fiducial marker seeds in prostate cancer patients. This detector does not require any prior knowledge of the seed positions. A software program has been developed that can visualize each image obtained directly from the $\mathrm{kV}$ XVI panel, draw a bounding box around the seeds and plot the centroids of the seeds in real-time.

Methods The detector was trained using a variety of datasets of fiducial marker seeds, that were initially labelled and used as ground truth. The validation and testing were performed on unseen XVI images. Software program was developed to display the projection images and predict the seeds using YOLO v2 and determine the centroids on each image while scanning the directory containing image files.

Results The fiducial marker seeds were successfully detected in $98 \%$ of images from all gantry angles, the variation in the position of the seed centroid was within $\pm 1 \mathrm{~mm}$. The percentage difference between the ground truth and the detected seeds was within the $3 \%$ tolerance. Conclusion The deep learning model to detect fiducial marker seeds in $\mathrm{kV}$ XVI panel images with no prior knowledge of their positions was successfully demonstrated. This is an ongoing project at the Princess Alexandra hospital and work is underway to extend it to other sites for tracking moving structures with minimal effort. The final aim is to incorporate it into clinical practice for use in radiotherapy treatment.

\section{O040 Surface image guided radiation therapy implementation}

\author{
J Lehmann ${ }^{1,2,3}$, T Pedersen ${ }^{1}$, J Wolf ${ }^{1}$, A Wilfert ${ }^{1}$
}

Department of Radiation Oncology, Calvary Mater Newcastle, Australia. (Joerg.Lehmann@calvarymater.org.au) [Presenting author]), (Therese.Pedersen@calvarymater.org.au), (Joshua.Wolf@calvarymater.org.au), (Alex.Wilfert@calvarymater.org.au). ${ }^{2}$ Institute of Medical Physics, University of Sydney, Australia. ${ }^{3}$ School of Mathematical and Physical Sciences, University of Newcastle, Australia

Introduction The use of optical methods to assess the patient surface is the basis of a range of commercial solutions for patient setup and monitoring in Radiation Therapy simulation and treatment. Following a market analysis by a multidisciplinary team our department has invested in a C-Rad Sentinel system and four C-Rad Catalyst+HD systems.

Method The commissioning is based on AAPM TG147 [1] with input from recent work [2]. This included the assessment of the integration of peripheral equipment, measurements of spatial reproducibility, evaluation of static and dynamic localization accuracy and the development of ongoing quality assurance procedures. Commissioning was tailored to the intended use of the systems, which includes DIBH and 4DCT for the Sentinel on a CT scanner and DIBH and SRS for the Catalyst+HD on linacs, in addition to patient setup and monitoring for a broader patient population. A Hexamotion phantom with a custom platform [3] was used to assess static and dynamic motion responses. For the field of view (FOV) evaluation a novel mapping method has been developed.

Results At the time of submission the Sentinel and one Catalyst+HD system had been installed, the commissioning measurements for the Sentinel were complete and commissioning of the Catalyst+HD had begun.

Sentinel warm up from a cold state showed isocentre drifts of $1.4 \mathrm{~mm}$ in the up-down direction and $2.3 \mathrm{~mm}$ in the sup-inf direction with stabilization at approximately 100 minutes. Warmup from the recommended standby was within $0.12 \mathrm{~mm}$.

The measured height of a stationary object on a moving CT couch fluctuated by about $1 \mathrm{~mm}$. Reconstruction of 4DCT images was flawless (Figs. 1, 2). 


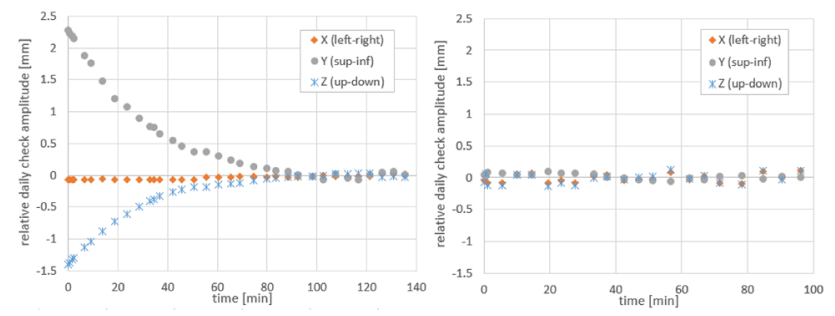

Figure 1 Drift of the Sentinel isocentre from a cold start (left) and from the recommended standby mode (right)

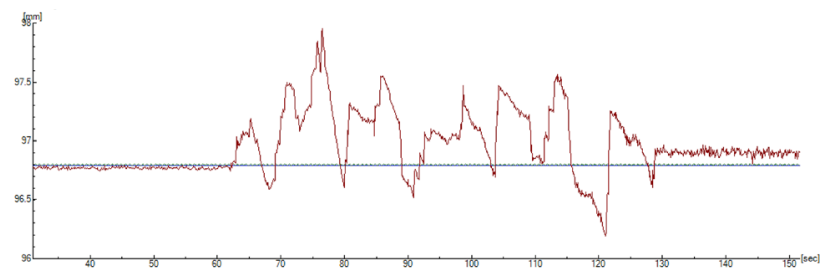

Figure 2 Relative height of a stationary object on the CT couch before $(<60$ s), during and after $(>130$ s) a custom, extended length CT scan

Conclusion Sentinel is ready for clinical use. As recommended, it should remain in standby overnight, with warmup needed in case of power outage.

Acknowledgements

Calvary Mater Newcastle is a reference site for C-Rad.

References

1. Twyla Willoughby, Joerg Lehmann, Jose A. Bencomo, et al "Quality assurance for nonradiographic radiotherapy localization and positioning systems: Report of Task Group 147" Med. Phys. 39 (4), April 2012

2. Jeremy D. P. Hoisak, Adam B. Paxton, Benjamin Waghorn, Todd Pawlicki (editors) "Surface Guided Radiation Therapy " CRC Press ISBN 978-1-1385-9349-7 2020

3. Guneet Kaur, Joerg Lehmann, Peter Greer, John Simpson "Assessment of the accuracy of truebeam intrafraction motion review (IMR) system for prostate treatment guidance", Australas Phys Eng Sci Med, https://doi.org/10.1007/s13246-019-00760-7, 2019

\section{O041 Comparison of three Realsense ${ }^{\mathrm{TM}}$ RGB-D cameras for measuring respiratory like motion of a surface}

\section{A L Fielding, J Kim}

Science \& Engineering Faculty, Queensland University of Technology (QUT), Brisbane, Australia, (a.fielding@qut.edu.au [Presenting Author])

Introduction Motion of the tumour during a radiotherapy treatment can compromise the clinical outcome for the cancer patient. To address this challenge, the suitability and performance of three different models of the Intel RealSense ${ }^{\mathrm{TM}}$ depth camera technology have been investigated as potential tools for measuring the respiratory-like motion of a surface. (a)

(b)
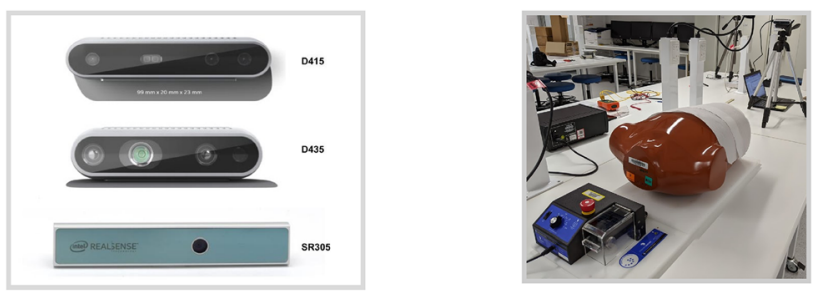

Figure 1 (a) The three Realsense $\mathrm{T}^{\mathrm{TM}}$ cameras used in the study. https://www.intelrealsense.com/ (b) the measurement set up for the thorax phantom (a)

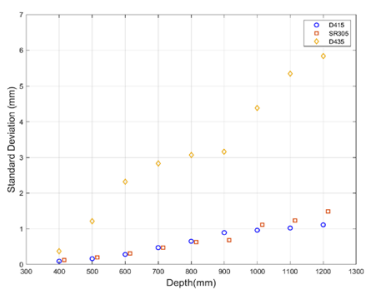

(c)

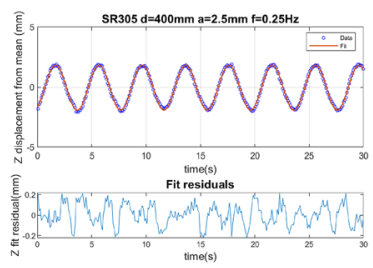

(b)

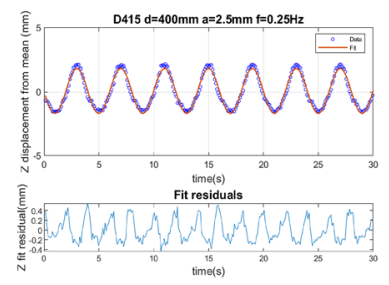

(d)

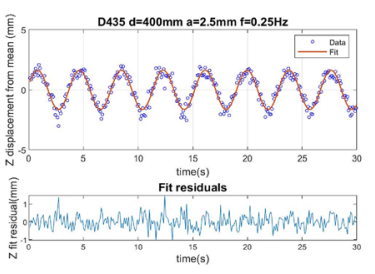

Figure 2 Comparison of the performance of the three cameras' for measuring distance to a surface. (a) Standard deviation of the measured depth vs depth over a 300 -frame acquisition for a stationary surface. (b) - (c) Measured displacements of the surface moving with sinusoidal motion of amplitude $2.5 \mathrm{~mm}$ and frequency $0.25 \mathrm{~Hz}$

Method Three different models of Intel Realsense ${ }^{\mathrm{TM}}$ depth camera (the D415, D435, and SR305) were used in this study (shown in Fig. 1a). Acquisitions were performed with in-house software developed using the MATLAB wrapper functionality of the Realsense ${ }^{\mathrm{TM}}$ SDK 2.0 at a frame rate of $30 \mathrm{fps}$ [1]. The precision of the camera depth data has been characterised as a function of the measurement distance up to 1.2 metres from a stationary and moving flat surface. Additional measurements, with the three cameras were performed for a moving thorax phantom to simulate a more clinical scenario, shown in Fig. 1b.

Results Figure 2(a) shows the standard deviation over 300 frames of the depth measurement as a function of measurement distance (depth) for a stationary surface. Figure $2 b-d$ shows examples, for the three cameras, of the measured displacements of a surface moving with a sinusoidal motion amplitude of $2.5 \mathrm{~mm}$ and frequency of $0.25 \mathrm{~Hz}$. Similar results for the three cameras were observed when measuring the motion of the thorax phantom surface.

Conclusion The D415 and SR305 cameras were able to measure the distance between the camera and surface with a similar precision that deteriorated as the distance increased. The D435 camera showed significantly worse precision with far higher levels of noise evident in the measured depth values. The D415 and SR305 cameras could be 
suitable for measuring patient surface motion during a radiotherapy treatment.

\section{References}

1. Fielding AL, Pandey AK, Jonmohamadi Y, Via R, Weber DC, Lomax AJ, et al. Preliminary study of the Intel RealSense ${ }^{\mathrm{TM}}$ D415 camera for monitoring respiratory like motion of an irregular surface. IEEE Sens J. 2020;1-1.

\section{O042 Intrafraction motion in Lung SBRT quantified through repeat $\mathrm{CBCT}$ imaging}

\author{
J Barber ${ }^{1,2}$, A Espinoza ${ }^{1}$, K West ${ }^{1}$, J R Sykes ${ }^{1,2}$ \\ ${ }^{1}$ Sydney West Radiation Oncology Network, Westmead \\ and Blacktown, NSW, Australia. ${ }^{2}$ Institute of Medical Physics, \\ University of Sydney, NSW, Australia. \\ (jeffrey.barber@health.nsw.gov.au [Presenting author]), \\ (anthony.espinoza@health.nsw.gov.au), \\ (katrina.west@health.nsw.gov.au), \\ (jonathan.sykes@health.nsw.gov.au)
}

Introduction To ensure accurate delivery for lung SBRT in our department, a mid-treatment CBCT is performed between VMAT arcs and image guidance performed, however FFF treatments significantly reduce delivery time. Analysis of intrafraction motion from CBCT performed at initial verification, mid-treatment and posttreatment was analysed to determine the necessity of mid-treatment verification, and if a time dependence for intrafraction motion exists. Method Image registration and couch positions were extracted from Aria Offline Review for a sequential 52 lung SBRT patients treated over 219 fractions. The registrations and applied corrections were analysed for magnitude and direction of drift across fractions, as well as correlated with timing of the image acquisition.

Results The mid-imaging confirmed patient position within tolerances of $2 \mathrm{~mm}$ in $88.5 \%, 3 \mathrm{~mm}$ in $94.5 \%$ and $4 \mathrm{~mm}$ in $97.7 \%$ of fractions. Of the 4 fractions with mid matches $>4 \mathrm{~mm}$, there was a clear reason for each shift on retrospective review. Median midimaging time adds 3.5 minutes. A linear fit suggested an average intrafraction movement time dependence of $0.2 \mathrm{~mm}$ per minute, but large variation was observed.

Conclusion Applying a $3 \mathrm{~mm}$ tolerance for intrafraction motion, midtreatment CBCT indicated 5.5\% fractions out of tolerance. The time required for performing and analysing the $\mathrm{CBCT}$ would be equivalent to delivering the second half of FFF treatment. As such, mid-treatment CBCT were removed from routine practice and reserved for patients with high likelihood of intrafraction motion.

\section{O043 Paediatric OBI planar imaging: where are we now?}

\section{J Miller, L Sim}

Radiation Oncology Princess Alexandra Raymond Terrace, Princess Alexandra Hospital, South Brisbane, Australia. (JulieAnne.Miller@health.qld.gov.au [Presenting author]), (Lucy.Sim@health.qld.gov.au)

Introduction Radiation Oncology Princess Alexandra Hospital Raymond Terrace (ROPART) is the primary provider of paediatric radiation therapy services in Queensland. The paediatric $\mathrm{kV}$ planar imaging protocols developed by British Columbia Cancer Agency, described by Rogerson [1], undergo constant adaption at ROPART.
The reasons for modifications include broadening the sites, optimising dose and improving the image quality.

Although there has been substantial development in radiology [2, 3] to optimise of exposures, the development of imaging protocols for paediatrics radiation therapy are not widely published. A review of ROPART exposure settings were compared with recently published low-dose protocols by Ryan et al. [4].

Method Low-dose protocols for paediatric IGRT was determined through a phantom study. Three anatomical sites, head and neck, thorax, and extremity were investigated. For each site the $\mathrm{kV}$ and $\mathrm{mAs}$ were adjusted until the image quality was adequate to perform image matching, as judged subjectively by a group of experienced radiation therapists. In addition, the relative dose comparison by site between ROPART and Ryan et al [4] protocols were assessed using RaySafe Unfors X2 with RF sensor at isocentre with a $20 \times 20 \mathrm{~cm}^{2}$ field.

Table 1 Dose of an orthogonal pair comparison of ROPART Adolescent setting and Ryan et al [4], with full fan Bowtie filter

\begin{tabular}{|c|c|c|c|c|}
\hline Site & $\begin{array}{l}\text { ROPART } \\
\text { (mGy) }\end{array}$ & $\begin{array}{l}\text { Ryan et al. [4] } \\
(\mathrm{mGy})\end{array}$ & $\begin{array}{l}\text { Dose } \\
(\%)\end{array}$ & Reduction \\
\hline Thorax & 0.48 & 0.76 & 37 & \\
\hline Abdomen & 0.12 & 0.61 & 80 & \\
\hline Pelvis & 0.11 & 0.47 & 77 & \\
\hline $\begin{array}{c}\text { Head and } \\
\text { Neck }\end{array}$ & 0.09 & 0.56 & 84 & \\
\hline
\end{tabular}

Table 2 Paediatric settings, for Varian Clinac iX linear accelerators equipped with $\mathrm{kV}$ On Board Imager (version 1.6) with full fan bowtie filter and small focal spot

\begin{tabular}{|c|c|c|c|c|c|}
\hline Site & Projection & Patient Size & $\mathrm{kV}$ & $\mathrm{mA}$ & $\mathrm{ms}$ \\
\hline \multirow[t]{3}{*}{ Thorax } & \multirow[t]{3}{*}{ Lateral } & Infant & 85 & 40 & 12.5 \\
\hline & & Child & 85 & 40 & 20 \\
\hline & & Adolescent & 85 & 100 & 100 \\
\hline \multirow[t]{3}{*}{ Thorax } & \multirow[t]{3}{*}{ Anterior } & Infant & 80 & 40 & 12.5 \\
\hline & & Child & 80 & 40 & 20 \\
\hline & & Adolescent & 80 & 40 & 32 \\
\hline \multirow[t]{3}{*}{ Abdomen } & \multirow[t]{3}{*}{ Lateral/ Anterior } & Infant & 85 & 40 & 12.5 \\
\hline & & Child & 85 & 40 & 20 \\
\hline & & Adolescent & 85 & 40 & 32 \\
\hline \multirow[t]{3}{*}{ Pelvis } & \multirow[t]{3}{*}{ Lateral } & Infant & 85 & 40 & 12.5 \\
\hline & & Child & 85 & 40 & 20 \\
\hline & & Adolescent & 85 & 40 & 32 \\
\hline \multirow[t]{3}{*}{ Pelvis } & \multirow[t]{3}{*}{ Anterior } & Infant & 80 & 40 & 12.5 \\
\hline & & Child & 80 & 40 & 20 \\
\hline & & Adolescent & 80 & 40 & 32 \\
\hline \multirow[t]{2}{*}{ Extremity } & \multirow[t]{2}{*}{ Lateral/ Anterior } & Child & 65 & 32 & 25 \\
\hline & & Adolescent & 65 & 50 & 25 \\
\hline \multirow[t]{3}{*}{ Head and Neck } & Lateral/ Anterior & $<10$ & 80 & 40 & 12.5 \\
\hline & Posterior & $10+$ & 90 & 50 & 20 \\
\hline & Lateral & $10+$ & 80 & 40 & 20 \\
\hline
\end{tabular}


Results The ROPARTs imaging protocols were all found to be less than the published exposure settings. Examples for Adolescent patients (aged 10 years and over) are shown in Table 1.

Conclusion There is a wide variation of treatment verification imaging exposure settings in published literature in radiation oncology. ROPARTs imaging protocols have been tailored to the department's needs and consist of a comprehensive list of sites and patient size. Implementation of the low-dose exposure table presented in this study should be considered by paediatric radiotherapy departments wishing to image gently.

\section{References}

1. Rogerson C (2013) ALARA and Pediatric Imaging in Radiation Therapy. 71st Annual General Conference of the Canadian Association of Medical Radiation Technologists

2. Damilakis J. (2016) Establishing and monitoring DRLS. Phys Med 32: 179-80.

3. Evans S. (2014) European diagnostic reference levels in paediatric imaging. Phys Med. 30: e14-5

4. Ryan J, Willis D (2020) Paediatric image-guided radiation therapy: determining and evaluating appropriate kilovoltage planar exposure factors for the Varian on-board imager. J Med Radiat Sci 67 (1): 16-24

\section{O044 Non-invasive cardiac ablation for refractory ventricular tachycardia: a case report}

\author{
N Hardcastle ${ }^{1}$, G Lee ${ }^{2}$, L Hall ${ }^{1}$, B Geary ${ }^{1}$, R Anderson ${ }^{2}$, G \\ Hanna $^{1}$, T Kron ${ }^{1}$, S Siva ${ }^{1}$ \\ ${ }^{1}$ Peter MacCallum Cancer Centre, Melbourne, VIC Australia. ${ }^{2}$ Royal \\ Melbourne Hospital, Melbourne, VIC Australia
}

Purpose Stereotactic ablative body radiotherapy (SABR) has recently emerged as a promising approach for treatment of refractory ventricular tachycardia [1]. We report Australia's first application of SABR for refractory ventricular tachycardia.

Methods and Materials A 75 year old man with severe, dilated cardiomyopathy with recurrent VT who had underwent three catheter ablation procedures was referred for SABR. Targeting was performed using a combination of invasive mapping from catheter ablation procedures, 12 lead ECGs, and using novel 3D non-invasive 252 electrocardiographic imaging (CardioInsight, Medtronic) process. A 4DCT radiotherapy simulation CT was performed with IV contrast. Target definition was performed by cardiologists and radiation oncologists in collaboration with international experts at University of Washington in St. Louis. A $3 \mathrm{~mm}$ planning target volume (PTV) expansion was applied to the target volume. A single fraction of 25 Gy was planned to cover $95 \%$ of the PTV, with sharp reduction of dose outside the PTV.

Results The target volume (right ventricle wall) was $17 \mathrm{~cm}^{3}$, with the treated PTV $47 \mathrm{~cm}^{3}$. The treatment was delivered with the presence of cardiologists, radiation oncologists with monitoring of the patient by cardiac electrophysiologists and anaesthetics team. Image guidance was performed using cone beam CT, using cardiac artery calcifications to assist with image registration. The treatment delivery time was 25 mins, which included two CBCTs prior to treatment and one mid-treatment, and 7 mins beam on time. There were no acute post-treatment toxicities observed.

Conclusion Single fraction SABR was successfully delivered to the right ventricle wall for treatment of refractory ventricular tachycardia. A strong inter-disciplinary and international collaboration was fundamental to the successful completion of the procedure. Further work will focus on spatial combination of treatment planning CT geometry with imaging and electrical mapping used for targeting.

\section{References}

1. Robinson CG, Samson PP, Moore KMS, Hugo GD, Knutson N, Mutic S, et al. Phase I/II Trial of Electrophysiology-Guided Noninvasive Cardiac Radioablation for Ventricular Tachycardia. Circulation. 2019 Jan 15;139(3):313-21.

\section{O045 Adopting a ${ }^{99 m}$ Tc-DTPA glomerular filtration rate estimation method and implementing a spreadsheet with real-time quality control feedback}

\section{S Gautam}

Department of Medical Physics, John Hunter Hospital, New Lambton Heights, NSW. (sijan.gautam@health.nsw.gov.au [Presenting Author])

Introduction GFR estimation using radiopharmaceuticals is prone to sample collection, counting and calculation errors, which are irreversible post-calculation, but easily rectifiable if identified in realtime. Results vary between radiopharmaceuticals, calculation methods and correction formulas. A new spreadsheet with real-time feedback based on quality control checks and a report with relevant age-specific normal ranges has been developed and implemented.

Method QC checks were based on departmental protocol for activities, timing and time sequence, and expected counts from standard activity. Tolerances were based on equipment precision with allowances for human error. Normal GFR ranges applicable to this calculation method were obtained by recalibrating published reference ranges and the compatibility with our patient cohort was assessed. 957 past reports were analysed and common errors were identified. The reported normal range was found to be significantly different from widely accepted publications. A presentation to staff was followed by active assistance in the initial months of implementation.

Results The most common errors such as typos, time-sequence errors and missing digits in counts have been completely eliminated. Technologists immediately rectify flagged data-entry fields before proceeding further. Any breach of sample counting tolerances have not yet been encountered. Taking all the tolerances into account, $<5.8 \%$ error in reproducibility has been estimated. Confidence in counting performance and quantification of reproducibility allow a significant dose reduction of $50 \%$ in adults and up to $87 \%$ in children. Conclusion The quoted normal range in our report, adapted to the method used, ensures reporting uniformity and increases referrer reliability to make informed clinical decision. Further data accumulation will allow stringent tolerances and evaluation of postcalculation QC metrics like volume of distribution and clearance halftime present in the spreadsheet. One challenge with transitioning to a radically new protocol in a large multi-site institution has been to get all staff to adapt to the changes simultaneously.

Acknowledgements I would like to acknowledge Thomas Greig for his help with data consolidation and Nicholas Hille for his support to implement this change in the department. 


\section{O046 An end-to-end deep learning based algorithm for delineating organs on low dose whole body CT scans for nuclear medicine dosimetry}

\section{D Pain, G J O'Keefe, G Rouvalis, S Gong}

${ }^{1}$ Department of Molecular Imaging and Therapy, Austin Health, Australia. (cameron.pain@austin.org.au [Presenting author]). (graeme.okeefe@austin.org.au), (george.rouvalis@austin.org.au), (sylvia.gong@austin.org.au)

Introduction Internal dosimetry is essential for determining thresholds for decreased organ function from therapies and quantifying radiation dose detriment to the public from diagnostic imaging. The process of calculating doses from functional imaging is time consuming, requiring accurate image segmentation and data analysis. The following work presents the development and implementation of an automated internal dosimetry application utilising convolutional neural network $(\mathrm{CNN})$ based image segmentation of low dose CT scans.

Method Two hundred unique whole body low dose CT images were manually segmented to create a training dataset containing binary masks of the lungs, liver, spleen, kidneys, brain, heart and vertebrae as ground truths. CNNs were constructed in Tensorflow1.14.0, trained with an augmented instance of the training dataset and utilised in a segmentation algorithm. The segmentation algorithm was incorporated into a dicom server framework running on an external network location which receives CT data as a PACS location and returns the segmentation in the form of a radiotherapy planning structure. An additional twenty whole body CT scans were segmented manually to evaluate the segmentation algorithm.

Results All segmentations for the twenty evaluation scans produced volumes within $90 \%$ of the manually segmented volume. The mean processing time of the twenty evaluation segmentations was (mean SD) seconds. Figure 1 slices of a whole-body CT scan segmented with the automated algorithm. Utilising a radiotherapy planning structure output allows the end user to dynamically edit the segmentation allowing for manual optimisation if required.

Conclusion An automated CNN based segmentation algorithm has been developed in a framework which is easily implemented clinically. The algorithm presented demonstrates a significantly more efficient alternative for dosimetric analysis. Further evaluation and packaging into a user-friendly application will be done in the immediate future.

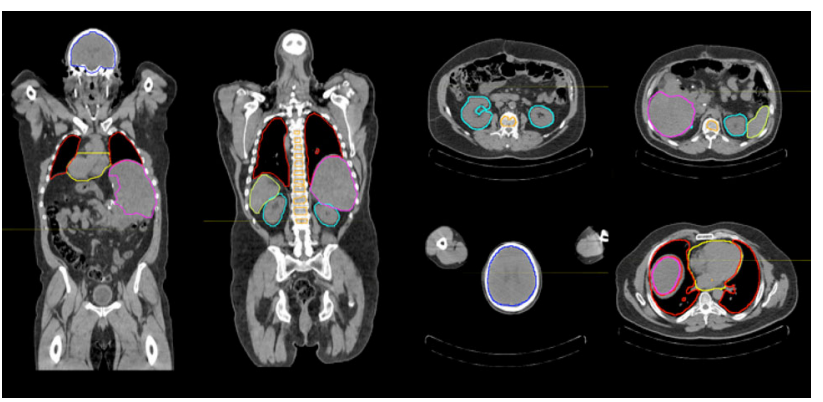

Figure 1 Example of an automatically segmented whole body CT
O047 Automatic segmentation of metastatic prostate lesions in PET/CT images using convolutional neural networks

J M Kendrick ${ }^{1}$, J Ong ${ }^{2}$, G M Hassan ${ }^{3}$, R Francis ${ }^{4}$, M McCarthy ${ }^{2}$, P Rowshanfarzad ${ }^{1}$, M A Ebert ${ }^{1,5}$

${ }^{1}$ Department of Physics, University of Western Australia, Australia. (21308325@student.uwa.edu.au), (Pejman.rowshanfarzad@uwa.edu.au), (Martin.Ebert@health.wa.gov.au). ${ }^{2}$ Department of Nuclear Medicine, Fiona Stanley Hospital, Perth, Australia. (jeremy.ong@health.wa.gov.au), (Michael.Mccarthy@health.wa.gov.au). ${ }^{3}$ Department of Computer Science \& Software Engineering, University of Western Australia. (ghulam.hassan@uwa.edu.au). ${ }^{4}$ Department of Nuclear Medicine, Sir Charles Gairdner Hospital, Perth, Australia. (Ros.Francis@health.wa.gov.au). ${ }^{5}$ Department of Radiation Oncology, Sir Charles Gairdner Hospital (SCGH), Perth, Australia

Introduction The application of radiomics-based predictive models of advanced prostate cancer using ${ }^{68} \mathrm{Ga}$-PSMA PET/CT images requires segmentation of metastatic prostate lesions. Manual segmentation is a time consuming and laborious process when there are tens or hundreds of lesions. The automation of this process could reduce user time required for this process, facilitate efficient treatment planning for patients undergoing radionuclide therapy as well as minimise inter-operator variability. This study aims to develop an automated segmentation framework using deep learning techniques for metastatic prostate lesions in whole body ${ }^{68} \mathrm{Ga}$-PSMA PET/CT images.

Method A local dataset of 256 patients with metastatic prostate cancer, who have had whole body ${ }^{68} \mathrm{Ga}$-PSMA PET/CT assessments, was analysed. A clinical expert manually delineated PSMA-avid lesions in each scan, which constituted the ground truth segmentation data that was subsequently used to train a convolutional neural network $(\mathrm{CNN})$. The metric of model evaluation was the Dice similarity coefficient (DSC), measuring the overlap between the model predictions and the ground truth manual delineations.

Results Preliminary results suggest the feasibility of whole-body PET/CT automated delineation using deep learning techniques. Utilising the MultiResUNet CNN model architecture [1], the best average DSC achieved thus far is $60.5 \%$, which is comparable to literature results [2]. The model will continue to be refined with a combination of pre-processing steps and optimised CNN architecture (Fig. 1).

Conclusion This novel research demonstrates the feasibility of automatically segmenting metastatic prostate lesions in whole body PET/CT images using deep learning techniques. This could facilitate efficient lesion detection and quantification, allowing medical imaging specialists to focus on interpretation and comparison tasks and optimising workflow, and can alleviate some of the laborious manual work of lesion detection.

\section{References}

1. Ibtehaz N, Rahman MS (2019) MultiResUNet: Rethinking the U-Net architecture for multimodal biomedical image
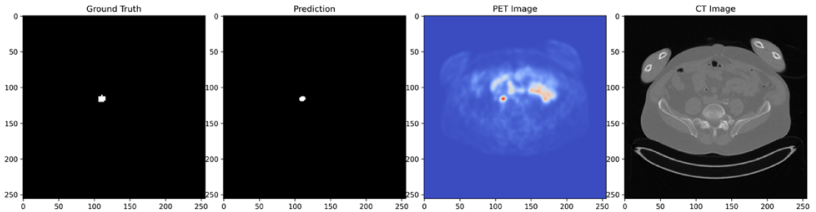

Figure 1 Demonstration of model prediction, with the corresponding ground truth label and PET/CT image 
segmentation. Neural Networks 121:74-87. https://doi.org/ 10.1016/j.neunet.2019.08.025

2. Zhao Y, Gafita A, Vollnberg B et al (2019) Deep neural network for automatic characterisation of lesions on ${ }^{68}$ Ga-PSMA-11 PET/ CT. European Journal of Nuclear Medicine and Molecular Imaging 47(3):603-13. https://doi.org/10.1007/s00259-01904606-y

\section{O048 Use of FET PET uptake dynamics for the spatial characterisation of glioblastoma}

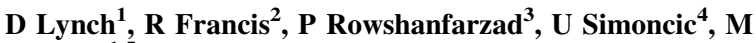 \\ A Ebert ${ }^{1,5}$ \\ ${ }^{1}$ School of Physics, Mathematics and Computing, University \\ of Western Australia, Australia. (21954738@student.uwa.edu.au \\ [Presenting author]). ${ }^{2}$ Department of Nuclear Medicine, Sir Charles \\ Gairdner Hospital, Perth, Australia. (roslyn.francis@uwa.edu.au). \\ ${ }^{3}$ Department of Physics, University of Western Australia, Australia. \\ (pejman.rowshanfarzad@uwa.edu.au). ${ }^{4}$ Faculty of Mathematics \\ and Physics, University of Ljubljana, Slovenia. \\ (urban.simoncic@ijs.si). ${ }^{5}$ Department of Radiation Oncology, Sir \\ Charles Gardiner Hospital, Perth, Australia. \\ (martin.ebert@health.wa.gov.au)
}

Introduction This study is being undertaken to utilise spatial dynamic characterisation of uptake of ${ }^{18}$ Fluoro-O-(2) fluoroethyl-Ltyrosine $\left({ }^{18} \mathrm{~F}\right.$-FET $)$ to generate biomarkers predictive [1] of glioblastoma (GBM) treatment outcome.

Method A Patlak graphical analysis technique was developed and tested on $24{ }^{18}$ F-FET positron emission tomography (PET) patient image data sets. The dynamic images were acquired from approximately five minutes post tracer injection, with images taken every minute for between 20-30 minutes in total. Allowing for the uptake flux (Ki) of the radiotracer into the brain tissue and time-to-peak (TTP) of tissue time activity curves to be calculated on a voxel-wise basis, forming 3D parametric maps which can further characterise cancerous regions of the brain. ${ }^{18}$ F-FET PET scans were performed on GBM patients with both post-resection/pre-radiochemotherapy and post-radiochemotherapy scans. The superior sagittal sinus vein of each brain scan was segmented using the earliest PET frames of high plasma-blood activity using 3D Slicer in order to generate an input function for each patient. Tissue time activity curves were extracted on a voxel-by-voxel basis in MATLAB, allowing a graphical analysis to be implemented and uptake flux and TTP maps of the brain to be calculated. The intra-patient stability of these maps was tested in 8 patients who had a third series of pre-radiochemotherapy scans taken a week apart by measuring mean square error statistics between the maps.

Results Maps of parameter uncertainty were generated by extracting confidence intervals and mapping their spatial variations. Voxel-level time-activity relationships were found to be strongly impacted by large amounts of image noise, greatly restricting the ability to derive both dynamics parameters as well as time-activity curve features (time-to-peak and categorised patterns).

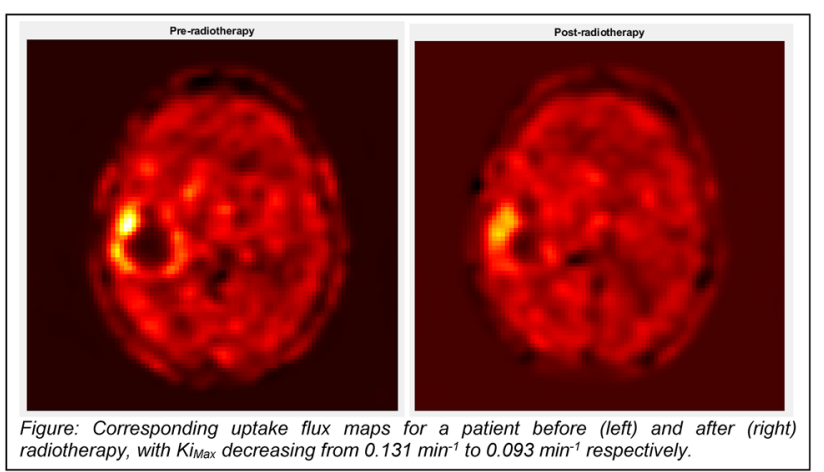

Conclusion The presented approach for calculating parametric maps of dynamic ${ }^{18}$ F-FET PET scans allowed for enhanced spatial characterisation of glioblastoma by highlighting areas of increased uptake flux and early TTP, with dynamic image noise being a major source of uncertainty.

\section{References}

1. Chan DL et al (2018). J Clin Neur, Vol. 58, Pg. 130-135.

\section{O049 Clinical benefits of managing motion in kidney SABR}

\section{Gaudreault ${ }^{1,2}$, T Kron ${ }^{1,2}$, S Siva ${ }^{2,3}$, N Hardcastle ${ }^{1}$}

${ }^{1}$ Department of physical sciences, Peter MacCallum Cancer Centre, Melbourne, Victoria 3051, Australia. ${ }^{2}$ Sir Peter MacCallum Department of Oncology, the University of Melbourne, Victoria 3010, Australia. (mathieu.gaudreault@ petermac.org [Presenting author]), (tomas.kron@petermac.org), (shankar.siva@petermac.org), (nick.hardcastle@ petermac.org). ${ }^{3}$ Division of Radiation Oncology,

Peter MacCallum Cancer Centre, Melbourne, Victoria 3051, Australia

Introduction Stereotactic ablative body radiotherapy (SABR) is a novel treatment for patients with inoperable renal cell cancer. We have previously demonstrated that delivered dose to kidney during SABR is associated with renal function decline [1]. Typically these patients have impaired kidney function, and occasionally a solitary kidney, therefore reductions of ipsilateral kidney dose is desirable. This study determines the reduction of ipsilateral kidney dose from elimination of respiratory motion in kidney SABR.

Method For twenty-nine kidney SABR patients, the gross tumour volume (GTV) was segmented on each phase of a phase-binned 4DCT. Tumour motion was obtained from the GTV centroid position on each phase. Motion managed (MM) plans were optimized and calculated to the GTV on the exhale phase. Non-motion managed (NMM) plans were optimised and calculated on the average of the 4DCT to an internal target volume (ITV). The NMM plan was copied ten times to the exhale phase, with isocentre corresponding to the tumour motion in that phase. The dose was calculated in each phase and averaged. Change in ipsilateral kidney volume receiving $50 \%$ of the prescription dose (V50\%) was assessed as a function of tumour motion amplitude (TMA). Pearson correlation coefficient was used in the statistical analysis.

Results The mean \pm st.dev TMA was $6.8 \pm 3.4,3.2 \pm 1.7,1.4 \pm 1.1 \mathrm{~mm}$ in sup-inf, ant-post and left-right directions respectively. A linear relationship between GTV to ITV volume difference and TMA was observed $\left(\mathrm{R}^{2}=-0.89 ; \mathrm{P}\right.$-value $\left.<0.001\right)$ as shown in Figure 1. A larger volume of non-tumour ipsilateral kidney was spared in MM plan compared with NMM plan (V50\% reduction of $11.0 \% / 4.6 \% / 0.7 \%$ 
$\mathrm{max} / \mathrm{median} / \mathrm{min})$. V50\% decrease is correlated with TMA as shown in Figure $2\left(\mathrm{R}^{2}=-0.67\right.$; P-value $\left.<0.001\right)$.

Conclusion Removing respiratory motion in kidney SABR reduces dose to the ipsilateral kidney, with the benefit increasing with increasing target motion.

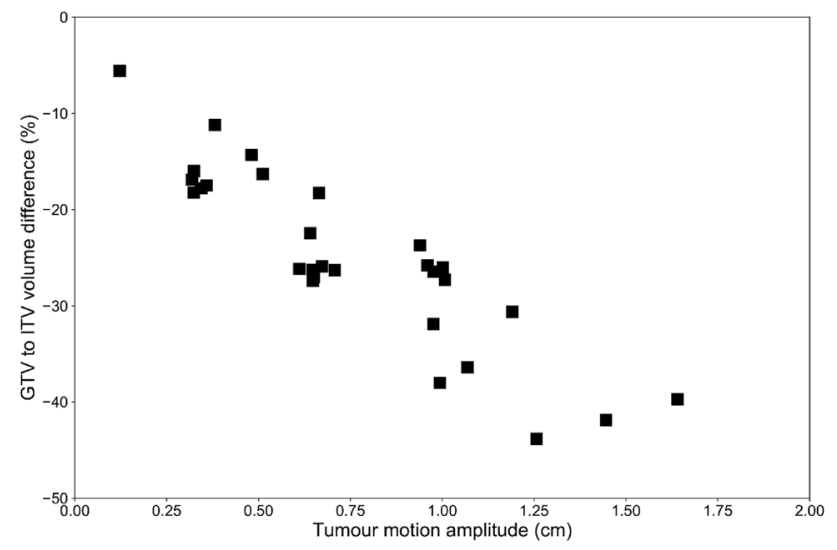

Figure $1 \mathrm{GTV}$ to ITV volume relative difference $(\%)$ as a function of tumour motion amplitude $(\mathrm{cm})$

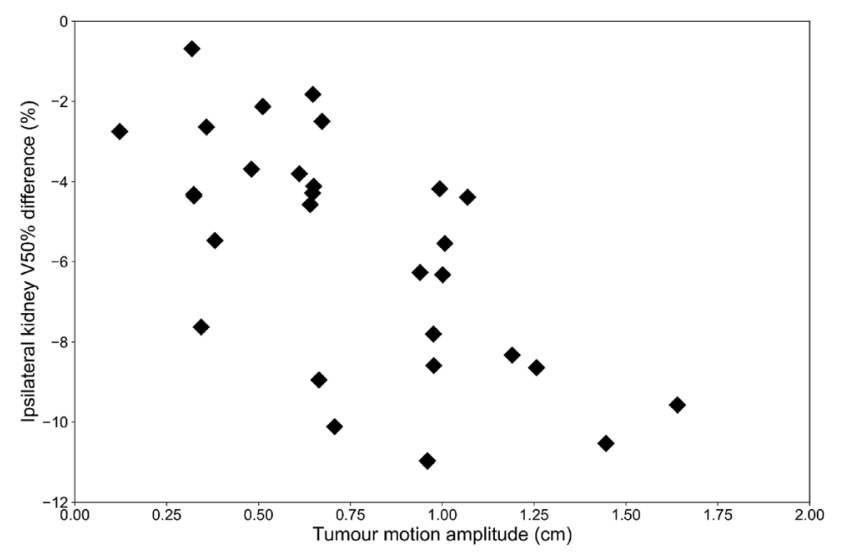

Figure 2 Ipsilateral kidney V50\% difference (\%) between MM plan and NMM plan as a function of tumour motion amplitude $(\mathrm{cm})$

\section{References}

1. Siva S, Jackson P, Kron T, Bressel M, Lau E, Hofman M, Shaw M, Chander S, Pham D, Lawrentschuk N, Wong LM, Goad J, Foroudi F (2016) Impact of stereotactic radiotherapy on kidney function in primary renal cell carcinoma: Establishing a doseresponse relationship. Radiotherapy and Oncology 118:540-546. http://dx.doi.org/10.1016/j.radonc.2016.01.027

\section{O050 Application of ICRU Report No. 91 recommendations for the Stereotactic Body Radiotherapy for Stage I Non-small-cell lung carcinoma (NSCLC) at Central West Cancer Care Centre (CWCCC)}

A Mishra, D Banjade, K Thuraisingam, S J Tan, B Newham, H Sheehan, A Renshaw

Department of Radiation Oncology, Central West Cancer Care Centre, Orange Hospital, Orange NSW 2800, Australia. (Ajeet.Mishra@health.nsw.gov.au [Presenting author])

Introduction It is anticipated that Stereotactic Body Radiotherapy (SBRT) is better tolerated and might lead to better overall survival compared to surgery for operable clinical stage I NSCLC. Although the workflow of lung SBRT appears to be similar to that for conventional radiotherapy, there are crucial distinctions in its delivery that require special attention. The aim of this paper is to address these challenges by applying the recommendations of ICRU Report No. 91 for implementation of SBRT technique for stage I NSCLC at CWCCC.

Method Recommendations of ICRU Report No. 91 were implemented for the dose reporting and Quality assurance of SBRT treatment plans. Pre-treatment Quality assurance tests include independent MU verification, point dose measurement, gamma analysis of dose distribution using EBT3 films and evaluation of dose distribution for PTV and OAR. Before treating every fraction, Winston-Lutz test and IGRT Quality Assurance tests were performed as per AAPM TG 142. A core group of multidisciplinary team members including RO, ROMP and RT is present at treatment to monitor the delivery of every fraction of SABR treatment.

Results We developed a clinical framework to address different components of SBRT process which include: patient assessment, simulation and immobilization, motion management, target and normal organ delineation, treatment planning, quality assurance, participation in external audit, treatment verification imaging and delivery, and patient follow-up. The 4D CT checklist and pre-treatment checklist for SBRT lung are shown in figure 1 and 2, respectively.

Conclusion SBRT is a resource intensive treatment technique and is specific to individual treatment centre. The effective and satisfactory transition from conventional radiotherapy practice to hypo-fractionated SBRT requires additional attention. Implementation of this cutting edge technology at CWCCC has made hypo-fractionated

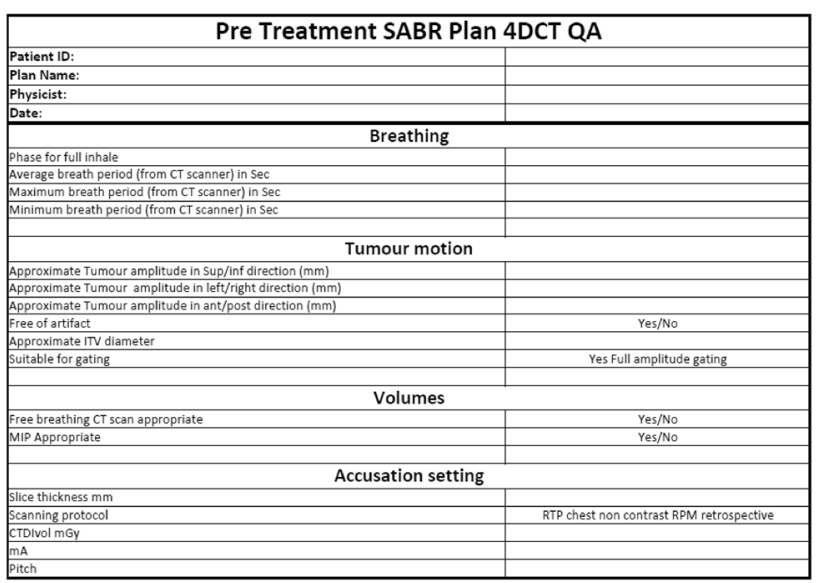

Figure 1 4DCT QA checklist 


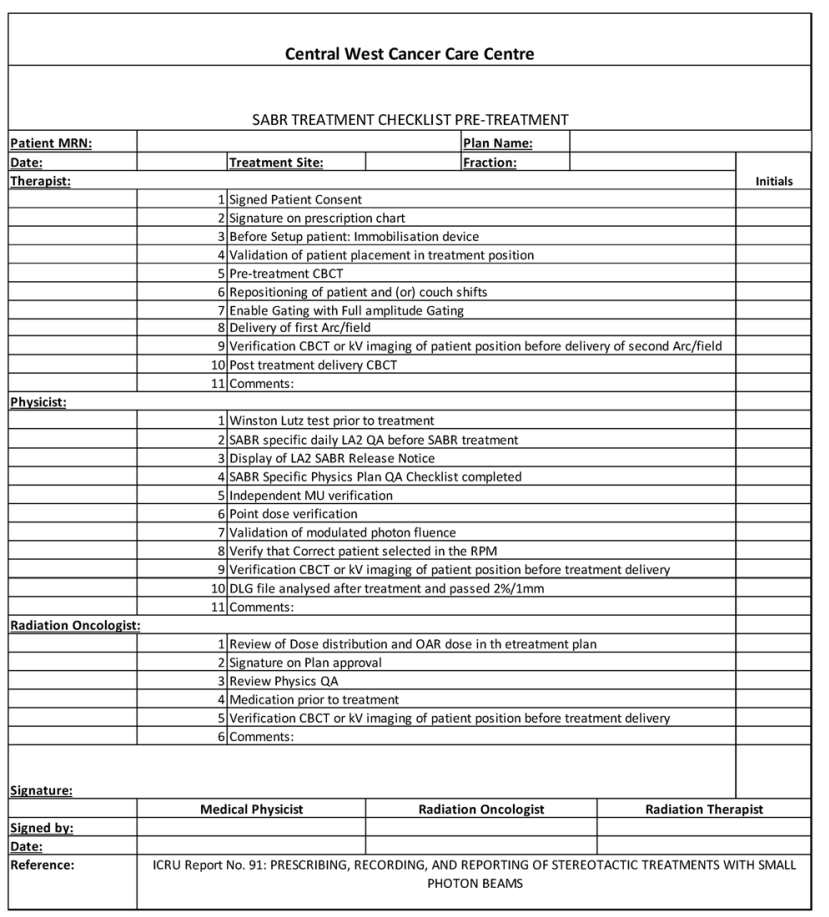

Figure 2 Pre-treatment SBRT lung checklist

Radiotherapy available to patients of regional and remote areas of Western NSW at their doorstep bringing equity in patient care at par with other metro cancer centres. This will enhance the SBRT utilization rate in this region thereby improving the patient survival rate of stage I NSCLC (Figs. 1, 2).

\section{O051 Initial experience with Brainlab Elements - Cranial SRS: moving from iPlan for single-lesion treatments}

\section{E C Cosgriff, A M Kejda, T Moodie}

Crown Princess Mary Cancer Centre, Westmead, Australia. (eireann.cosgriff@health.nsw.gov.au [Presenting author]), (alannah.kejda@health.nsw.gov.au), (trevor.moodie@health.nsw.gov.au)

Introduction Radiosurgery treatments at the Crown Princess Mary Cancer Centre include a static-field non-coplanar isocentric technique (iPlan) for patients with 1 to 4 brain lesions or a dynamic conformal arc non-coplanar single-isocentre technique for multiple metastases $(>4)$. The service has recently expanded with the addition of the Elements $^{\mathrm{TM}}$ Cranial SRS application from Brainlab, which is designed for the planning of single-lesion treatments with noncoplanar volumetric modulated arc therapy (VMAT) optimisation, allowing both dose and dose rate/gantry speed modulation. A retrospective planning study was undertaken to evaluate the change in plan quality when adopting this new technique.

Method A selection of static-field single-lesion plans were replanned in Cranial SRS (v. 1.5) and compared using the following metrics [1]: monitor units, homogeneity index, maximum dose to prescription dose (MDPD) ratio, prescription isodose volume divided by the treatment volume (PITV), the Paddick conformity index, the RTOG coverage [2] (minimum dose to treatment volume divided by the prescription dose), the gradient index, and the volume of normal brain receiving $12 \mathrm{~Gy}\left(\mathrm{~V}_{12 \mathrm{~Gy}}\right)$. Doses to the brainstem and optic structures were also considered.

Results PTV volumes were in the range of 0.22 to $1.23 \mathrm{cc}$ planned for $21 \mathrm{~Gy}$ in a single fraction prescribed to the $80 \%$ isodose. The average increase in monitor units for the VMAT plans was 6\%. There were increases in the average homogeneity index, Paddick conformity index (change in average of 0.76 to 0.81 ), and RTOG coverage, and a decrease in the PITV value. There were no substantial changes in the MDPD and minimal changes to the gradient index. Preliminary results indicate a decrease in $\mathrm{V}_{12}$ Gy of around $40 \%$ (average volume change of $2.8 \mathrm{cc}$ to $1.6 \mathrm{cc}$ ).

Conclusion Non-coplanar VMAT-based treatment of single-lesions offers increased conformity and decreased normal brain doses in comparison to a non-coplanar static-field technique.

\section{References}

1. Salkeld AL, Wang W, Nahar N, Hau EKC, Sykes J, Moodie T, Thwaites DI (2018). EP-2175: Improvement in radiosurgical plan dosimetry with implementation of a quality assurance program. Radiother Oncol 127:S1201-S1202. https://doi.org/10.1016/ S0167-8140(18)32484-8.

2. Shaw E, Kline R, Gillin M, Souhami L, Hirschfeld A, Dinapoli R, Martin L (1993) Radiation therapy oncology group: Radiosurgery quality assurance guidelines. Int $\mathrm{J}$ Radiat Oncol Biol Phys 27:1231-1239. https://doi.org/10.1016/0360-3016(93)90548-a

\section{O052 An attempt to quantify the effect on output factor of a sub-millimetre variation in the size of a small radiation field}

\section{G Biasi ${ }^{1,2}$, L Muñoz ${ }^{1,3}$, J Bucci ${ }^{4}$, M Jackson ${ }^{1,5}$, M Lerch $^{1}$, M Petasecca $^{1}$, P Metcalfe ${ }^{1}$, A B Rosenfeld ${ }^{1}$ \\ ${ }^{1}$ Centre for Medical Radiation Physics, University of Wollongong, NSW, Australia. ${ }^{2}$ Peter MacCallum Cancer Centre, Melbourne, VIC, Australia. (gbiasi@uow.edu.au).3GenesisCare Flinders Private Hospital, Bedford Park, SA, Australia. (luis.munoz@genesiscare.com). ${ }^{4}$ St. George Cancer Care Centre, St George Hospital, Kogarah, NSW, Australia. ${ }^{5}$ University of New South Wales, Kensington, NSW, Australia}

Introduction In megavoltage photon beams, dosimetry of radiation fields $\leq 30 \mathrm{~mm}$ presents several challenges [1]. Besides the uncertainty on positioning the dosimeter [2], and on correction factors required to relate its reading to dose [1], uncertainty in the effective

Table $1 \mathrm{OPF}$ as a function of effective (measured) and equivalent field size

\begin{tabular}{lllllllll}
\hline $\begin{array}{l}\text { Field } \\
{[\mathrm{mm}} \\
\times \mathrm{mm}]\end{array}$ & $\begin{array}{l}\mathrm{EF} \\
{[\mathrm{mm}]}\end{array}$ & $\begin{array}{l}\text { OPF Field } \\
{[\%]}\end{array}$ & $\begin{array}{l}\text { EF } \\
{[\mathrm{mm}} \\
\times \mathrm{mm}]\end{array}$ & $\begin{array}{l}\text { OPF } \\
{[\mathrm{mm}]}\end{array}$ & $\begin{array}{l}\text { Field } \\
{[\mathrm{mm}} \\
\times \mathrm{mm}]\end{array}$ & $\begin{array}{l}\text { EF } \\
{[\mathrm{mm}]}\end{array}$ & $\begin{array}{l}\text { OPF } \\
{[\%]}\end{array}$ \\
\hline $5.9 \times 4.9$ & 5.4 & 41.4 & - & - & - & $20.0 \times 19.9$ & 19.9 & 81.9 \\
$6.2 \times 5.1$ & 5.6 & 43.6 & $10.4 \times 10.2$ & 10.3 & 69.1 & $20.2 \times 19.9$ & 20.0 & 82.3 \\
$6.3 \times 4.9$ & 5.6 & 43.3 & $10.6 \times 10.1$ & 10.3 & 69.2 & - & - & - \\
$6.4 \times 5.1$ & 5.7 & 45.9 & - & - & - & - & - & - \\
\hline
\end{tabular}


size of the field can have a big effect on measurements of output factor (OPF). Here, we attempted to quantify how OPF changes as a function of sub-millimetre variations in the effective size of a small field.

Method We eliminated the influence of other compounding uncertainties (on positioning and on correction factor) by using a 2D solidstate array, the Duo, which has a $0.2-\mathrm{mm}$ resolution over $5.2 \times 5.2$ $\mathrm{cm}^{2}$, and does not require corrections [3,4]. We placed the Duo at 90-cm source-to-surface distance, 10-cm depth in Solid Water, and aligned it with the central axis of an Elekta Versa HD using a 5-mm square field [5]. We then delivered 6 MV FFF beams, collimated with an Agility multi-leaf collimator in the cross-plane and with the Y-jaw in the in-plane, applying sub-millimetre shifts to the collimators and measuring OPF each time. The equivalent field was defined as $\mathrm{EF}=\sqrt{ }(\mathrm{A} \times \mathrm{B})$, where $\mathrm{A}$ and $\mathrm{B}$ were the cross-plane and in-plane FWHM.

Results OPF are in Table 1, normalized to the $10 \times 10 \mathrm{~cm}^{2}$ reference field. In a 5.7-mm EF, a cross-plane change of $0.2 \mathrm{~mm}$ resulted in a $5.0 \%$ variation in OPF, whereas in a $20.0-\mathrm{mm}$ EF the same change resulted in a $0.5 \%$ variation. In fields equal to the same $\mathrm{EF}$, collimated with interchanged configuration of MLC/Y-jaw, OPF varied by $0.7 \%$ in the $5.6-\mathrm{mm} \mathrm{EF}$, and by $0.1 \%$ in the $10.3-\mathrm{mm} \mathrm{EF}$.

Conclusion OPF in small stereotactic beams should be reported as a function of effective field size [5]. Here, we proposed that the effective field size should be reported with sub-millimetre accuracy, in both cross-plane and in-plane directions.

Acknowledgements We would like to thank GenesisCare for access to their linacs.

\section{References}

1. IAEA. Technical Reports Series No. 483 - Dosimetry of Small Static Fields Used in External Beam Radiotherapy An International Code of Practice for Reference and Relative Dose Determination. 2017.

2. Lechner W, Georg D, Palmans H. An analytical formalism for the assessment of dose uncertainties due to positioning uncertainties. Med Phys 2020;47:1357-63. https://doi.org/10.1002/mp.13991.

3. Shukaili K Al, Petasecca M, Newall M, Espinoza A, Perevertaylo VL, Corde S, et al. A 2D silicon detector array for quality assurance in small field dosimetry: DUO. Med Phys 2017;44:628-36. https://doi.org/10.1002/mp.12060.

4. Shukaili K Al, Corde S, Petasecca M, Pereveratylo V, Lerch M, Jackson M, et al. "Characterization of ELEKTA SRS cone collimator using high spatial resolution monolithic silicon detector array.” J Appl Clin Med Phys 2018;19:114-24. https:// doi.org/10.1002/acm2.12345.

5. ICRU. Report 91. J Int Comm Radiat Units Meas 2017;14:1-160. https://doi.org/10.1093/jicru/ndx017.

\section{Deep learning image reconstruction - commissioning and validation}

\section{Williams, P Barnes}

Department of Radiology, Austin Health, VIC. (chris.williams@austin.org.au [Presenting author]), (Peter.barnes@austin.org.au)

Introduction Deep learning methods have proven their value in radiology, from segmentation to equipment maintenance. Recently, the prospect of utilising deep learning to aid image reconstruction has been commercially realised. Manufacturer claims include features such as improved noise texture and better performance at low doses. However, deep learning image reconstruction (DLR) is fundamentally different from other reconstruction technology and introduces the potential for novel artefacts [1], so appropriate care should be taken in assessing and commissioning it.

Method The properties of the DLR algorithm in comparison to iterative reconstruction (IR) and filtered back projection (FBP) were assessed using a CatPhan 600. Noise images were evaluated by subtracting images reconstructed using each algorithm from an averaged image acquired by performing repeated FBP, which is taken as the ground truth. These images are used to evaluate the noise power spectrum (NPS), as well as illustrate differences between the algorithms, demonstrating any artefacts introduced by DLR. The modulation transfer function was also measured to assess spatial resolution.

Results The DLR algorithm demonstrated improved noise frequency and similar spatial resolution. The relationship between noise and dose was similar for IR and FBP, however the response of DLR at very low doses appeared less consistent than for the established algorithms. Inspection of the subtracted images showed that the DLR differed from the FBP standard by more than IR on average (mean HU difference of 0.07 for DLR vs 0.01 for IR). Furthermore, the outline of structures of the CatPhan were visible in the subtracted images (Figure 1), indicating that there are circumstances in which spatial fidelity is subtly affected.

Conclusion The value of the DLR algorithm was demonstrated, and echoes results found by other investigators [2]. However, the importance of comprehensive quality assurance when using novel algorithms was also clear.

\section{References and Acknowledgements}

1. Antun V, Renna F, Poon C, Adcock B, Hansen AC. On instabilities of deep learning in image reconstruction and the potential costs of AI. Proc Natl Acad Sci. Published online May 11, 2020:201907377. https://doi.org/10.1073/pnas.1907377117

2. Benz DC, Benetos G, Rampidis G, et al. Validation of deeplearning image reconstruction for coronary computed tomography angiography: Impact on noise, image quality and diagnostic

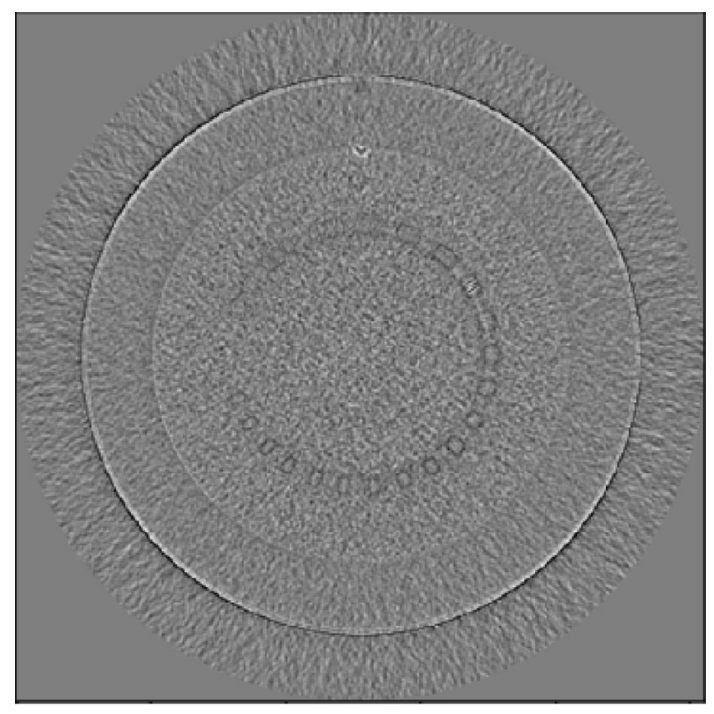

Figure 1 Appearance of structure outlines in a DLR - FBP subtraction 
accuracy. J Cardiovasc Comput Tomogr. 2020;(January):1-8. https://doi.org/10.1016/j.jcct.2020.01.002

The software used in this investigation was provided as a free trial by GE Healthcare.

\section{O054 Optimization of imaging dose in patients undergoing image guided radiotherapy using CBCT images}

\section{Khaled Alqahtani ${ }^{1}$, Christopher Noble ${ }^{2}$, Prabhakar Ramachandran $^{2}$}

${ }^{1}$ King Faisal Specialist Hospital \& Research Centre, Riyadh, Saudia Arabia. (kalqahtani32@kfshrc.edu.sa). ${ }^{2}$ Radiation Oncology, Princess Alexandra Hospital, Ipswich Rd, Brisbane, Australia.

(Christopher.Noble@health.qld.gov.au).

(Prabhakar.Ramachandran@health.qld.gov.au [Presenting author])

Introduction Image Guided Radiotherapy (IGRT) provides the ability to accurately target the radiation to the tumour volume and to minimise the dose to the surrounding normal tissue. Cone Beam Computed Tomography (CBCT) is the most common modality used in IGRT and although the imaging dose is small compared to the therapeutic dose it is delivered to a large volume of normal tissue and should be minimized.

Method The current work investigates the possibility of reducing the imaging dose from CBCT by modulating the mAs during an acquisition to match the patient anatomy. The current hardware in clinical CBCT systems does not allow the modulation of the mAs during a scan and therefore a framework for simulating this via software processing of the projection data was established. CBCT projection datasets were acquired on an Elekta Synergy XVI system at different mAs values. A Python script was then used to combine different proportions of the high and low mAs projections into a new dataset that could then be reconstructed on the XVI system.

Results Figure 1 shows a typical slice in the 3D volumetric image of a Brainlab Pelvis phantom after image reconstruction in XVI. The image on the left shows the reconstruction with $100 \%$ low mAs projections. The image on the right shows the reconstruction with $100 \%$ high $\mathrm{mAs}$ projections. The central image was reconstructed with $50 \%$ each of the high and low mAs projections. Quantitative analysis of CBCT reconstructions of a Catphan phantom showed a linear trend in the low contrast visibility with the proportion of high $\mathrm{mAs}$ projections used in the image reconstruction.

Conclusion The current framework has been shown to be an effective tool for exploring the possibility of reducing the imaging dose from CBCT. It has been demonstrated that it is possible to effectively balance the reduced low contrast visibility with reduced imaging dose by modulating the mAs during the acquisition scan. It has also been demonstrated that new metrics are needed to quantify the artefacts that may be introduced with modulated mAs schemes.
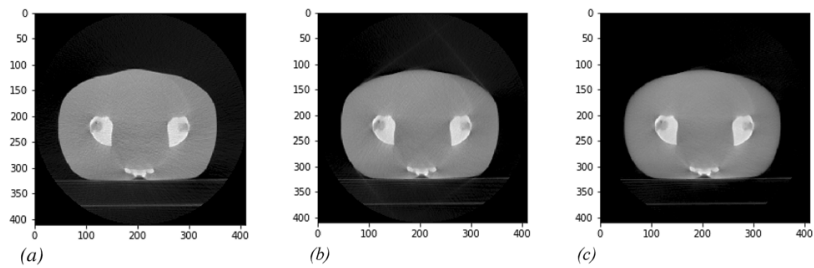

Figure 1 Pelvis phantom images reconstructed in XVI for; (a) low $\mathrm{mAs}$, (b) combined $50 \% \mathrm{mAs}$ and (c) high $\mathrm{mAs}$
O055 Efficient projection based method for metal artefact reduction in cone beam CT

\section{Hatim Almutairi ${ }^{1}$, Christopher Noble ${ }^{2}$, Prabhakar Ramachandran ${ }^{2}$}

${ }^{1}$ Medical Physics Department, Prince Mohammed bin Abdulaziz Hospital, Riyadh, Saudi Arabia. (almutairihm@pmah.med.sa). ${ }^{2}$ Radiation Oncology, Princess Alexandra Hospital, Ipswich Rd, Brisbane, Australia. (Christopher.Noble@health.qld.gov.au [Presenting author]), (prabhakar.Ramachandran@health.qld.gov.au)

Introduction Cone Beam Computed Tomography (CBCT) is the most common modality used in Image Guided Radiotherapy (IGRT). Metal Artefacts in CBCTs can dramatically reduce the accuracy of image registration in IGRT which can in turn reduce the ability to accurately target the tumour volume and minimise the dose to the surrounding normal tissue. It is therefore vital to develop methods for the accurate and efficient reduction of metal artefacts in CBCT.

Method The currently proposed method extends the projection-based method of Zhang et al [1]. The key improvements in the current methodology is the automatic segmentation of the metal shadows, the use of a highly efficient algorithm for the in-painting of the metal shadow and the addition of a space-carving technique to contour the metal object. The method was tested using a water phantom with high density metal inserts. The CBCTs were acquired on an Elekta Synergy XVI system. Images reconstructed with and without metal artefact reduction were compared with the ground truth images that were acquired with no metal objects.

Results Figure 1 shows a typical slice in the 3D volumetric image of the water phantom after image reconstruction in XVI. The image on the left shows the original reconstruction with severe metal artefacts. The image on the right shows the corresponding slice in the reconstructed image after metal artefacts reduction. The contours of the metal object derived from space carving have been added. The central image was acquired with no metal objects present and with the metal object contours added was used as the ground truth data for quantitative analysis.

Conclusion The current methodology has been shown to be highly effective in reducing metal artefacts in CBCT. It can be easily incorporated into current clinical workflows with no additional operator interventions or significant increase in time. The addition of the space carving technique allows the use of the metal object contours for image registration.

\section{References}

1. Y. Zhang, L. Zhang, X. R. Zhu, A. K. Lee, M. Chambers, and L. Dong, "Reducing metal artifacts in cone-beam CT images by preprocessing projection data," Int. J. Radiat. Oncol. Biol. Phys., vol. 67, no. 3, pp. 924-932, Mar. 2007, https://doi.org/10.1016/ j.ijrobp.2006.09.045.

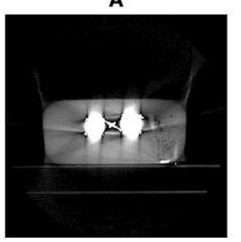

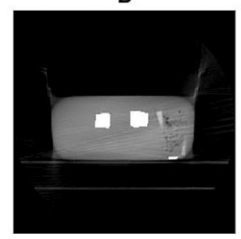

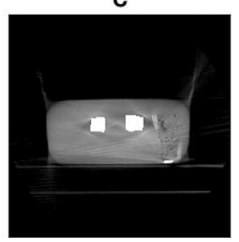

Figure 1 Slice in the reconstructed CT image of the phantom: A) uncorrected image showing significant metal artefacts B) ground truth, image without the coins present combined with metal contours, C) corrected image with in-painting of the metal shadow 


\section{O056 Evaluating an EGS_CBCT based CBCT scatter removal pipeline}

\author{
S Hegarty ${ }^{1}$, J Korte ${ }^{2,3}$, N Hardcastle ${ }^{2}$, R Franich ${ }^{1}$ \\ ${ }^{1}$ RMIT University, Melbourne Australia. \\ (s3654771@ @student.rmit.edu.au), (rick.franich@ rmit.edu.au). ${ }^{2}$ Peter \\ MacCallum Cancer Centre, Melbourne, Australia. ${ }^{3}$ Department \\ of Biomedical Engineering, School of Engineering, University \\ of Melbourne, Melbourne Australia. (james.korte@petermac.org), \\ (nick.hardcastle@petermac.org)
}

Introduction Cone Beam Computed Tomography (CBCT) scans are prone to negative effects from scattered radiation, resulting in incorrect Hounsfield Units (HU) [1]. Monte Carlo (MC) based scatter correction has been shown to resolve such issues [1, 2]. In such methods, scatter contribution in each projection is determined using $\mathrm{MC}$ simulation of projections, allowing acquired $\mathrm{CBCT}$ projections to be corrected. In this study we evaluated the impact of various methods of performing scatter correction with MC generated scatter contribution.

Method A pipeline for MC scatter generation and open-source CBCT reconstruction was created using EGSnrc and RTK. A Fan-beam CT (Philips Brilliance) and CBCT (Varian TrueBeam) of an anthropomorphic phantom were acquired. Primary and scatter simulated projections were produced from the fan-beam CT. The scatter was scaled via a ratio of the acquired projection to the $\mathrm{MC}$ equivalent (primary + scatter) and removed. Methods of this included scaling the scatter with a per-pixel ratio, the mean ratio of pixels including the phantom in the projection, and the mean ratio of all pixels.

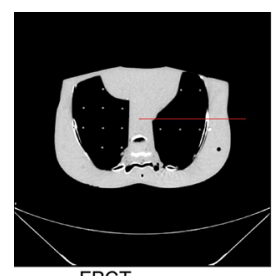

- $\mathrm{FBCT}$

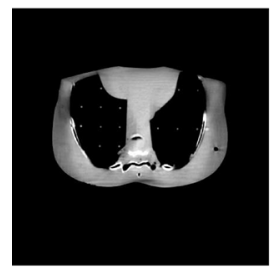

b) Per Pixel Scale

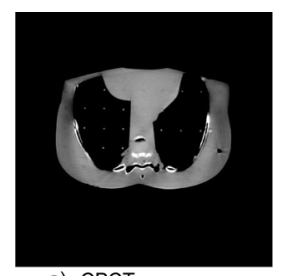

a) $\mathrm{CBCT}$

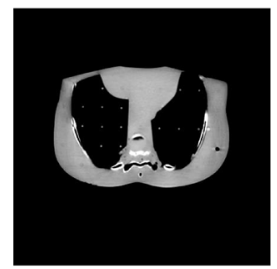

c) Partial Image Scale

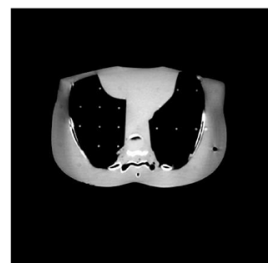

d) Full Image Scale
Figure 1 The ideal FBCT image (a), original CBCT image (b) and each of the corrected images (c-e). The same windowing has been applied to each image

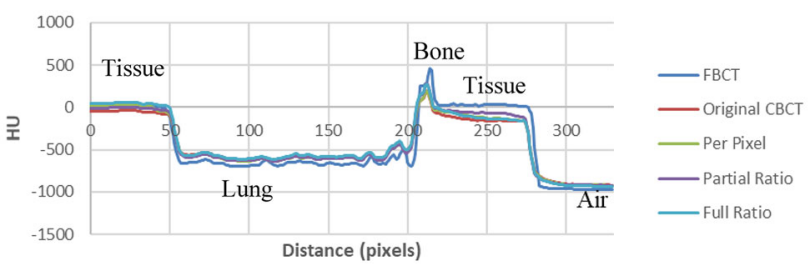

Figure 2 Line profile through each of the corrected images, the original CBCT image and the ideal FBCT image. Location of the line profile is marked on 1 a)
Results Figure 1 contains images of the fan-beam CT, original CBCT and scatter-corrected CBCT from each method. Partial image correction resulted in improved $\mathrm{HU}$ consistency at the phantom periphery. This can be seen in the line profiles of Figure 2. Optimal scatter correction has been achieved via partial ratio scaling, due to improved image uniformity. The HU difference from the centre to the periphery was reduced from $106 \mathrm{HU}$ (original CBCT) to $57 \mathrm{HU}$ with the partial ratio correction.

Conclusion A MC based scatter correction pipeline has been implemented. Scaling the scatter with a ratio calculated from part of the projection increased image uniformity and $\mathrm{HU}$.

\section{References}

1. Thing, R.S., et al., Hounsfield unit recovery in clinical cone beam $C T$ images of the thorax acquired for image guided radiation therapy. Physics in Medicine \& Biology, 2016. 61(15): p. 5781.

2. Mainegra-Hing, E., Fast monte carlo calculation of scatter corrections for cbct images. Journal of Physics: Conference Series, 2008. 102(1): p. 012017.

\section{O057 Cone-beam CT scatter correction:} a convolutional neural network approach for correcting head \& neck scans

\author{
B Rusanovi, M Sabet ${ }^{2}$, M A Ebert ${ }^{2}$, G Mukwada ${ }^{2}$ \\ ${ }^{1}$ Department of Physics, University of Western Australia, Australia. \\ (21310238@student.uwa.edu.au). ${ }^{2}$ Department of Radiation \\ Oncology, Sir Charles Gairdner Hospital, Australia. \\ (mahsheed.sabet@health.wa.gov.au), \\ (martin.ebert@health.wa.gov.au), \\ (godfrey.mukwada@health.wa.gov.au)
}

Introduction Cone-beam CTs (CBCT) are routinely used for patient setup prior to therapeutic beam delivery. Extending CBCT use toward dose accumulation and adaptive radiotherapy necessitates more accurate CBCT HU reproduction. As a proof-of-concept, this study aims to demonstrate how deep learning based on phantom data can be used for CBCT scatter correction in patient images.

Method Four anthropomorphic phantoms were scanned on a CBCT and CT. The subsequent cone-beam projections (CBp) were collected along with the reconstructed CT scan. Scatter correction is performed by estimating the cone-beam scatter-only signal via the method used by Nui [1]. For this, CBp are reconstructed using the FDK [2] reconstruction implementation in RTK, yielding CBCT_uncor. The $\mathrm{CT}$ is registered to $\mathrm{CBCT}$ _uncor, then forward projected using the CBCT geometry to yield virtual CT projections (vCT). Since vCT is assumed to be scatter-free, scatter signals are extracted by subtracting vCT from $\mathrm{CBp}$, yielding pSCA. For training a convolutional neural network $(\mathrm{CNN}), \mathrm{CBp}$ is used as the input and pSCA as the target. A modified version of ScatterNet [3] is utilized to train in 2D on a total of 2003 image pairs. Hence the network can estimate scatter signals from input $\mathrm{CBp}$. For testing, patient head and neck $\mathrm{CBp}$ data is passed through the network. Corrected CBCTs (CBCT_cor) are acquired by subtracting pSCA from $\mathrm{CBp}$, then reconstructed using the scattercorrected CBp.

Results The referenced scatter correction method reduced the mean absolute error (MAE) from $201 \mathrm{HU}$ to $74 \mathrm{HU}$ for phantom data. CNN derived scatter-corrected projections are yet to be generated for analysis.

Conclusion The referenced scatter-correction method has significantly improved the HU accuracy of phantom data. Given the nature of machine learning, CBCT_cor for patient images can, at best, approximate an MAE of $74 \mathrm{HU}$. 


\section{References/Acknowledgements}

1. Nui T (2010) Shading correction for on-board cone-beam CT in radiation therapy using planning MDCT images. Medical Physics 37(10):5395

2. Rit S (2014) The reconstruction toolkit (RTK), an open source cone-beam CT reconstruction toolkit based on the insight toolkit (ITK). J Phys Conf Ser 489:012079

3. Hansen D (2018) ScatterNet: A convolutional neutal network for cone-beam CT intensity correction. Medical Physics 45(11):4916

\section{Determination of cone beam computed tomography quality assurance tolerances using process control charts}

\section{J Harwood, K Legge}

Medical Physics and Radiation Engineering, Canberra Health Services, Australia. (jeffharwoodromp@gmail.com [Presenting Author])

Introduction Image-guided radiation therapy (IGRT) is an essential component of modern radiotherapy. Cone-beam computed tomography $(\mathrm{CBCT})$ is used to verify patient position and monitor changes in patient anatomy. Protocols provide guidance on the required quality assurance to ensure CBCT systems are capable of providing clinically useful images [1-3]. It has been reported that protocol-based quality assurance (QA) tolerances are not reliable at predicting clinical image quality issues [4].

CBCT technologies and applications undergo rapid development, meaning that historical QA tolerances may no longer be applicable. Additionally, many protocols instruct the user to compare to baseline data, but do not provide guidance on what variation from baseline is acceptable. Control charts can be used to monitor quality and identify out-of-control results. This work presents a novel approach to determining CBCT QA tolerances that address the above issues using process control charts.

Method We obtained 50 CBCT scans of a CATPHAN 604 phantom over a period of 14 days on a Varian Truebeam using standard Head

Table 1 Control limits for Head Protocol.

\begin{tabular}{|c|c|c|c|c|c|c|}
\hline Parameter & $\begin{array}{l}\text { HU } \\
\text { Lin- } \\
\text { earity } \\
\text { (HU) } \\
\text { \{acrylic }\end{array}$ & $\begin{array}{l}\text { Geo- } \\
\text { metric } \\
\text { node } \\
\text { spacing } \\
(\mathrm{mm})\end{array}$ & $\begin{array}{l}\text { Slice } \\
\text { thick- } \\
\text { ness } \\
\text { (nom- } \\
\text { inal } 2 \\
\text { mm) }\end{array}$ & $\begin{array}{l}\text { Low } \\
\text { contrast } \\
\text { reso- } \\
\text { lution } \\
\text { (size } \\
\text { in } \mathrm{mm} \text { ) }\end{array}$ & $\begin{array}{l}\text { High } \\
\text { contrast } \\
\text { reso- } \\
\text { lution } \\
\text { (lp/cm } \\
\text { or } \\
\text { MTF } \\
50 \%)\end{array}$ & $\begin{array}{l}\text { Unifor- } \\
\text { mity } \\
\text { (HU } \\
\text { or } \\
\text { unifor- } \\
\text { mity } \\
\text { index) }\end{array}$ \\
\hline $\begin{array}{l}\text { Tolerance } \\
\qquad(\mathrm{CPQR})\end{array}$ & Reproducible & Reproducible & N/A & Reproducible & $\leq 5$ & Reproducible \\
\hline $\begin{array}{l}\text { Tolerance } \\
\text { (AAPM) }\end{array}$ & Baseline & 2 & N/A & Baseline & Baseline & Baseline \\
\hline $\begin{array}{l}\text { Tolerance } \\
\quad \text { (Varian } \\
\text { IPA) }\end{array}$ & 50 & 0.5 & N/A & 15 & $\begin{array}{l}\leq 6 \\
\quad \text { (head } \\
\text { scan) }\end{array}$ & 30 \\
\hline $\begin{array}{l}\text { Upper } \\
\text { control } \\
\text { limit }\end{array}$ & $157(8)$ & 50.0 & 1.94 & N/A & 0.45 & 2.73 \\
\hline $\begin{array}{c}\text { Centre line } \\
\text { (mean } \\
\text { value) }\end{array}$ & $149(0)$ & 49.97 & 1.87 & N/A & 0.44 & 0.47 \\
\hline $\begin{array}{l}\text { Lower control } \\
\text { limit }\end{array}$ & $140(9)$ & 49.93 & 1.80 & N/A & 0.43 & -1.78 \\
\hline
\end{tabular}

and Thorax protocols. The data sets were analysed using pylinac [5]. We produced control charts using QATrack+ [6].

Results A comparison of control limits and protocol and manufacturer tolerances for the Head protocol in Table 1.

Conclusion Use of process control charts to determine QA limits for CBCT scans results in more specific limits when compared with published protocols, ensuring that scan quality and clinical utility can be more effectively monitored with routine QA.

\section{References}

1. Fontenot JD, Alkhatib H, Garrett JA, et al (2014) AAPM Medical Physics Practice Guideline 2.a: Commissioning and quality assurance of X-ray-based image-guided radiotherapy systems. J Appl Clin Med Phys 15:3-13. https://doi.org/10.1120/ jacmp.v15i1.4528

2. Bissonnette J-P (2018) COMP report: CPQR technical quality control guidelines for accelerator-integrated cone-beam systems for verification imaging. J Appl Clin Med Phys 19:9-12. https:// doi.org/10.1002/acm2.12302

3. Varian TrueBeam Installation Product Acceptance IPA-HT-2XICP-H November 2018.

4. Manger RP, Pawlicki T, Hoisak J, Kim G (2019) Technical Note: Assessing the performance of monthly CBCT image quality QA. Med Phys 46:2575-2579. https://doi.org/10.1002/mp.13535

5. Zaila A, Adili M, Bamajboor S (2016) Pylinac: A toolkit for performing TG-142 QA related tasks on linear accelerator. Phys Medica 32:292-293. https://doi.org/10.1016/j.ejmp.2016.07.122

6. Studinski R, Taylor R, Angers C, et al (2014) SU-E-T-103: Development and Implementation of Web Based Quality Control Software. Med Phys 41:246-246. https://doi.org/10.1118/ 1.4888433

\section{O059 International consensus data for high energy photon $k_{\mathbf{Q}}$ based on current measured and modelled data}

M D Hanlon ${ }^{1}$, P Andreo ${ }^{2}$, C P Oliver ${ }^{1}$, P D Harty ${ }^{1}$, V T Takau ${ }^{1}$, D J Butler ${ }^{1}$

${ }^{1}$ Primary Standards Dosimetry Laboratory, ARPANSA, Australia. (Maximilian.Hanlon@arpansa.gov.au [Presenting author]), (Chris.Oliver@arpansa.gov.au), (Peter.Harty@arpansa.gov.au), (Viliami.Takau@arpansa.gov.au), (Duncan.Butler@arpansa.gov.au). ${ }^{2}$ Karolinska University Hospital and Karolinska Institutet, Stockholm, Sweden. (pedro.andreo@ki.se)

Introduction The IAEA is reviewing TRS-398 [1], the absorbed dose-to-water dosimetry protocol used in Australia for external beam radiotherapy. As part of the update to high energy photon beams, the IAEA invited several groups including ARPANSA to contribute measured and Monte Carlo (MC) modelled data on the beam quality correction factor $\left(\mathrm{k}_{\mathrm{Q}}\right)$. This data forms a consensus dataset, which will likely form the basis of updated published $\mathrm{k}_{\mathrm{Q}}$ data in the update of TRS-398. As an interim measure, the consensus data has been provided as a separate publication. [2]

Method $\mathrm{k}_{\mathrm{Q}}$ values were determined from both $\mathrm{MC}$ simulations and direct calibration measurements. Data was generated for 23 cylindrical chamber types, consisting of 725 MC calculated and 179 measured data points, of which ARPANSA submitted 24 measured $\mathrm{k}_{\mathrm{Q}}$ data values across 8 chambers and $90 \mathrm{MC} \mathrm{k}_{\mathrm{Q}}$ values across 9 chambers. A fit to the equation:

(1) $k_{Q}\left(T P R_{20,10}\right)=\frac{1+\exp \left(\frac{a-0.57}{b}\right)}{1+\exp \left(\frac{a-T P R_{20,10}}{b}\right)}$ 


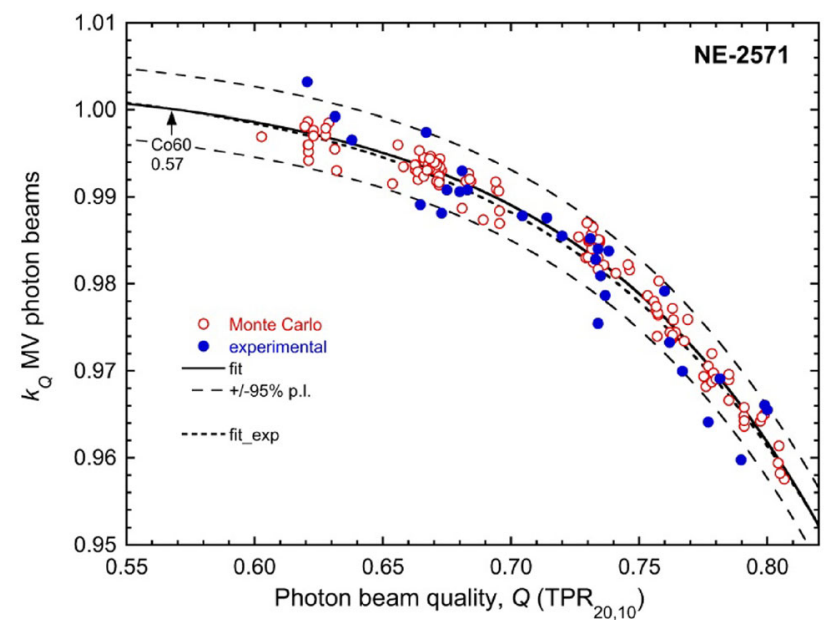

Figure 1 Values of $\mathrm{k}_{\mathrm{Q}}$ for the NE-2571 ionisation chamber, combining $126 \mathrm{MC}$ and 28 measured values. The solid fit line represents a fit to all data with $95 \%$ C.I. (long dashed lines), and the small dashed line represents a fit to all experimental data. Figure taken from Andreo et al. [2]

using the combined data set of MC and measured values was performed for each chamber, created a set of parameters, $a$ and $b$, that allows for the calculation of $\mathrm{k}_{\mathrm{Q}}$ at other beam qualities.

Results The consensus data of $\mathrm{k}_{\mathrm{Q}}$, combining measured and $\mathrm{MC}$ values, for the NE-2571 Farmer-type ionisation chamber is shown in Figure 1.

Consensus $\mathrm{k}_{\mathrm{Q}}$ data was generated for each of the 23 chambers. The consensus $\mathrm{k}_{\mathrm{Q}}$ data in both a tabular form as in TRS-398, and as a set of chamber specific parameters $a$ and $b$, as in equation 1 . The combined standard-uncertainty in the new $\mathrm{k}_{\mathrm{Q}}$ values is $0.62 \%$.

Conclusion A new consensus dataset for the beam quality correction factor $\mathrm{k}_{\mathrm{Q}}$ in high energy photons has been created. It consists of values from many international research groups and standards laboratories. The consensus data will likely form the basis of published $\mathrm{k}_{\mathrm{Q}}$ values in the updated protocol TRS-398.

\section{Acknowledgements}

The authors would like to acknowledge the many authors that contributed to the publication, and in turn the many more that contributed towards the published data. The authors of the paper not included as authors on the abstract are: D T Burns, R P Kapsch, M McEwen, S Vatnitsky, C E Andersen, F Ballester, J Borbinha, F Delaunay, P Francescon, Mirzakhanian, B Muir, J Ojala, M Pimpinella, M Pinto, L A de Prez, J Seuntjens, L Sommier, P Teles, J Tikkanen, J Vijande and K Zink

\section{References}

1. Andreo, P. et al (2000). Absorbed dose determination in external beam radiotherapy: an international code of practice for dosimetry based on standards of absorbed dose to water. IAEA TRS-398, 242.

2. Andreo, P. et al (2020). Determination of consensus kQ values for megavoltage photon beams for the update of IAEA TRS398. Physics in Medicine \& Biology, 65(9), 095011.
O060 Impact of the adoption of ICRU 90 on x-ray air kerma calibrations at ARPANSA

\section{D Hanlon ${ }^{1}$, C P Oliver ${ }^{1}$, T E Bailey ${ }^{1}$, J E Lye ${ }^{2}$, D J Butler ${ }^{1}$}

${ }^{1}$ Primary Standards Dosimetry Laboratory, ARPANSA, Australia. (Maximilian.Hanlon@arpansa.gov.au [Presenting author]), (Chris.Oliver@arpansa.gov.au), (Tracy.Bailey@arpansa.gov.au), (Jessica.Lye@arpansa.gov.au), (Duncan.Butler@arpansa.gov.au). ${ }^{2}$ Australian Clinical Dosimetry Service, ARPANSA, Australia

Introduction ARPANSA maintains two free air chamber (FAC) primary standards for low and medium energy x-rays. The low energy FAC (LEFAC) and medium energy FAC (MEFAC) are used over an energy range of $10-100 \mathrm{kVp}$ and $40-320 \mathrm{kVp}$ respectively. All x-ray air kerma calibrations offered by ARPANSA are traceable to these FACs.

Due to the adoption of the ICRU 90 recommendations by the CCRI(I) in 2018, the correction factors of each chamber changes, which in turn affects calibration factors of secondary standards calibrated at ARPANSA. Here, we present this change in calibration factors due to the adoption of ICRU 90.

Method Both the LEFAC and MEFAC were modelled in egs_fac [1] and validated against prior Monte Carlo models using monoenergetic source inputs and the same cross sections. [2]

Once the Monte Carlo models were validated, the simulations were rerun with renormalised photoelectric effect cross sections, as per ICRU 90. The correction factors were calculated by convolving the monoenergetic data with the spectra of the beams used in calibration. The new correction factor, $\mathrm{k}_{\mathrm{ii}} \mathrm{k}_{\mathrm{w}}$, was also incorporated.

Results Both the new LEFAC and MEFAC models showed good agreement to previous models, with differences either within uncertainty or attributable to previous assumptions made for efficiency.

The adoption of ICRU 90 data had a small effect on the product of correction factors for both the LEFAC and MEFAC. The LEFAC correction factor had a decrease between 0.2 to $0.4 \%$, depending on beam energy. The MEFAC correction factor showed a change of $0.4 \%$ to $+0.1 \%$.

Conclusion The correction factors for the FAC primary standards at ARPANSA have been recalculated, incorporating adoptions due to ICRU 90. While the small changes that are seen affect calibrations performed at ARPANSA, the differences are within the overall uncertainty budget of the calibration service.

\section{References/Acknowledgements}

1. Mainegra-Hing, E., Reynaert, N., \& Kawrakow, I. (2008). Novel approach for the Monte Carlo calculation of free-air chamber correction factors. Medical physics, 35(8), 3650-3660.

2. Lye, J. E., Butler, D. J., \& Webb, D. V. (2009). Monte Carlo correction factors for the ARPANSA kilovoltage free-air chambers and the effect of moving the limiting aperture. Metrologia, 47(1), 11.

\section{O061 Considerations in replacing ARPANSA's primary standard for absorbed-dose-to-water - graphite or water calorimetry?}

P D Harty ${ }^{1}$, D J Butler ${ }^{\mathbf{1}}$, M D Hanlon ${ }^{1}$, C P Oliver ${ }^{\mathbf{1}}$, I M Williams
${ }^{\mathbf{2}}$
${ }^{1}$ Primary Standards Dosimetry Laboratory, ARPANSA, Australia.
(Peter.Harty@arpansa.gov.au [Presenting author]), (Duncan.But-
ler@arpansa.gov.au), (Maximilian.Hanlon@ arpansa.gov.au), (Chris.
Oliver@arpansa.gov.au). ${ }^{2}$ Chief Medical Radiation Scientist,
ARPANSA, Australia. (Ivan.Williams@arpansa.gov.au)
Introduction ARPANSA maintains a graphite calorimeter primary
standard for absorbed-dose-to-water for ${ }^{60} \mathrm{Co}$ photons and linac 
megavoltage photon beams. With the prospect of proton radiotherapy in Australia, it is time to consider replacing our calorimeter with a version capable of measuring in both photon and proton beams. Some overseas primary standard laboratories have already upgraded, with both graphite and water options used. The relative merits are worth considering.

Method We have explored graphite and water calorimetry through the scientific literature [1], and experimented at the Australian Synchrotron, where graphite, graphene and water calorimeters have been tested in high-intensity beams.

Results Graphite has the advantage of a low specific heat capacity of $0.7 \mathrm{~Gy} /{ }^{\circ} \mathrm{C}$ compared to $4.2 \mathrm{~Gy} /{ }^{\circ} \mathrm{C}$ for water, with much greater temperature rise for graphite for the same photon fluence, allowing sensitive measurements at lower-dose-rate ${ }^{60} \mathrm{Co}$ beams. However, graphite requires conversion from absorbed-dose-to-graphite to absorbed-dose-to-water by Monte Carlo calculation or other method, introducing extra uncertainty. Conversely, water has the advantage of realising absorbed-dose-to-water directly. A water calorimeter is more massive, and therefore less portable. Both require correction factors, and both require embedded thermistors for heating/temperature measurement. These thermistors sit in the beam and are subject to output changes at the onset and after irradiation. This effect is known in calorimeter studies in $\mathrm{kV}$ beams (see [1], Figure 6) and is attributed to radiation induced current in the thermistors and leads. Data on this effect will be shown from our calorimeter studies at the Synchrotron. Conclusion Internationally both graphite and water calorimeters are used as primary standards, providing robustness to the international average determination of absorbed-dose-to-water. If ARPANSA chooses water calorimetry for a new primary standard while retaining graphite for comparison, robustness will be added to ARPANSA's capability to accurately measure absorbed-dose-to-water for present and future radiotherapy modalities in Australia.

\section{References}

1. Renaud, J. et al (2020). Absorbed dose calorimetry. Phys. Med. Biol. 65 05TR02.

\section{O062 Magnetic field correction factors via a cross- calibration using a conventional linac}

\author{
J Begg ${ }^{1,2,3}$, Urszula Jelen ${ }^{4}$, Gary Liney ${ }^{1,2,3,4}$, Lois \\ Holloway ${ }^{1,2,3,4,5,6}$
}

${ }^{1}$ Department of Medical Physics, Liverpool and Macarthur Cancer Therapy Centre, Sydney, Australia. ${ }^{2}$ Ingham Institute for Applied Medical Research, Sydney, Australia. ${ }^{3}$ South Western Sydney Clinical School, School of Medicine, University of New South Wales, Sydney, Australia. (jarrad.begg@health.nsw.gov.au [Presenting author]). ${ }^{4}$ St Vincent's Clinic Sydney, GenesisCare, Sydney, Australia. (urszula.jelen@ health.nsw.gov.au). ${ }^{4}$ Centre for Medical Radiation Physics, University of Wollongong, Wollongong,

Australia. (gary.liney@health.nsw.gov.au). ${ }^{5}$ Radiation Physics Lab, University of Sydney, Sydney, Australia. ${ }^{6}$ Institute of Medical Physics, School of Physics, University of Sydney, Sydney, Australia. (lois.holloway@health.nsw.gov.au)

Introduction Magnetic field correction factors, $\mathrm{k}_{\mathrm{B}}$, are required for dosimetry in magnetic fields. Current methods for determining $k_{B}$ include: transfer from water calorimeters or chemical dosimetry, measurements during magnet ramp up/down or monte-carlo simulations. This work aims to validate a methodology for measuring $\mathrm{k}_{\mathrm{B}}$ via measurements using a conventional linac.

Methods $\mathrm{k}_{\mathrm{B}}$ is a ratio of the absorbed dose to water calibration factor in the magnetic field to $0 \mathrm{~T}$ at the same beam quality $\left(\mathrm{N}_{\mathrm{DwQ}}^{\mathrm{B}} / \mathrm{N}_{\mathrm{DwQ}}^{0 \mathrm{~T}}\right)$. $\mathrm{k}_{\mathrm{B}}$ contains $\mathrm{i}$. dose to water ratio in the magnetic field to $0 \mathrm{~T}\left(\mathrm{D}_{\mathrm{w}}^{\mathrm{B}} / \mathrm{D}_{\mathrm{w}}^{0 \mathrm{~T}}\right)$ and ii. measured charge ratio of a dosimeter at $0 \mathrm{~T}$ to the magnetic field $\left(\mathrm{M}^{0 \mathrm{~T}} / \mathrm{M}^{\mathrm{B}}\right)$. This work proposes a cross-calibration between a conventional linac and a MR-Linac (MRL) to determine $\mathrm{k}_{\mathrm{B}}$. Charge ratios between a microdiamond and PTW30013 Farmer were measured at 0 and $1 \mathrm{~T}$ positions on the Australian MRL and on a conventional linac with the effective point of measurement at $10 \mathrm{~cm}$ depth in solid water. These measurements enabled: 1 . A calculation between a conventional linac and MRL at OT to determine if the change in beam quality will impact the result $\left(\left[\mathrm{M}_{\mathrm{FC}} / \mathrm{M}_{\mathrm{uD}}\right]^{\text {OT-- }}\right.$ $\left.{ }_{\mathrm{C}}^{\mathrm{Conv}}\left[\mathrm{M}_{\mathrm{uD}} / \mathrm{M}_{\mathrm{FC}}\right]^{\mathrm{OT}-\mathrm{MRL}}\right) ; 2$. A calculation between 0 and $1 \mathrm{~T}$ on the MRL to determine the expected $\mathrm{k}_{\mathrm{B}}\left(\left[\mathrm{M}_{\mathrm{FC}} / \mathrm{M}_{\mathrm{uD}}\right]^{\text {OT-MRL }} \times\left[\mathrm{M}_{\mathrm{uD}} /\right.\right.$ $\left.\left.\mathrm{M}_{\mathrm{FC}}\right]^{1 \mathrm{~T}}\right)$; 3. A calculation between 0T on a conventional linac and 1T on the MRL, to determine the $\mathrm{k}_{\mathrm{B}}$ via the proposed methodology $\left(\left[\mathrm{M}_{\mathrm{FC}} / \mathrm{M}_{\mathrm{uD}}\right]^{\text {0T-Conv }} \times\left[\mathrm{M}_{\mathrm{uD}} / \mathrm{M}_{\mathrm{FC}}\right]^{1 \mathrm{~T}}\right)$.

Results and Conclusion Results between the conventional linac and OT position was $0.999 \pm 0.004$ indicating no change due to beam quality. $\mathrm{k}_{\mathrm{B}}$ calculated via measurements at 0 and $1 \mathrm{~T}$ on the MRL and via $0 \mathrm{~T}$ on a conventional linac and $1 \mathrm{~T}$ on the MRL was $0.991 \pm 0.007$. These results are consistent with monte carlo simulations for the Farmer [1]. This work validated the methodology for measuring $k_{B}$ via measurements on a conventional linac. Future work will investigate applicability to perpendicular MRLs.

References

1. Spindeldreier et al, PMB 62(16):6708, 2017

\section{Post irradiation leakage in a 2D ionization chamber array}

\section{Christopher Noble, Prabhakar Ramachandran}

Radiation Oncology, Princess Alexandra Hospital, Ipswich Rd, Brisbane, Australia. (Christopher.Noble@ health.qld.gov.au [Presenting author]), (Prabhakar.Ramachandran@health.qld.gov.au)

Introduction The Octavius SRS1000 ion chamber array was acquired in 2016 and has been routinely used with the Octavius 4D phantom for patient specific QA measurements. Over time an increasing number of ion chambers showed signs of leakage currents that could be seen as spikes during the acquisition of the standard $12 \times 12 \mathrm{~cm}$ warmup field. When the detector is functioning normally this field is flat and hence any spikes can be discerned at this point whereas it is more difficult to distinguish this during measurements of patient plans which can be highly modulated. Method A retrospective analysis of PSQA results from 2016 until early 2020 was performed. 3156 X $12 \times 12 \mathrm{~cm}$ warmup fields were located for this period. The cumulative dose for each ion chamber is stored as a function of time at $0.2 \mathrm{~s}$ intervals during the acquisition. A Python script was written to read in these files and analyse the response of the ion chambers. The ion chamber readings during the beam on time and immediately after were investigated.
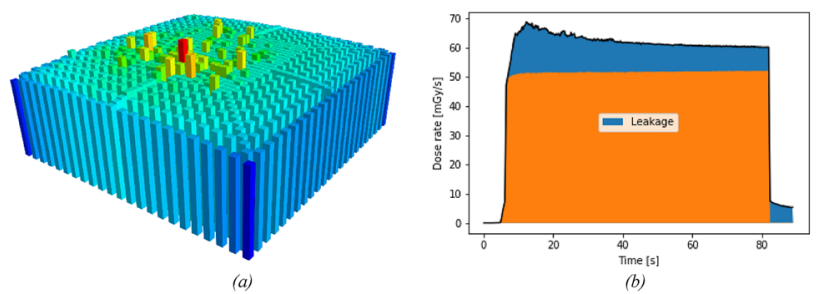

Figure 1 SRS1000 Octavius Ion chamber readings for a $12 \times 12$ warmup field. a) Large spikes in what should be a flat field b) Instantaneous dose rate reading for a leaking ion chamber in the SRS1000 detector array 
Results Figure 1a shows the cumulative ion chamber readings for the SRS1000 array recorded during a $12 \times 12 \mathrm{~cm}$ warmup field. The $\mathrm{x}$ and $\mathrm{y}$ coordinates correspond to the position of the ion chambers in the array and the height of the bars corresponds to the absorbed dose. Several spikes can be seen in the densely packed central region of the array. Figure $1 \mathrm{~b}$ shows the instantaneous dose rate measured for one of the ion chambers that showed leakage both during the beam and post irradiation.

Conclusion The deterioration in performance of the PTW Octavius SRA1000 detector array has been shown to be related to an increase in post-irradiation leakage. The current warmup fields combined with some data processing can effectively monitor the level of post-irradiation leakage in the detector array and can be used in a preventative maintenance program to ensure the validity of patient specific quality assurance measurements and the safe delivery of radiotherapy to patients.

\section{O064 MOSFET calibration factors for clinical use in radiotherapy}

\section{Peng ${ }^{1}$, D Hossain ${ }^{1}$, P Narayanan ${ }^{1}$, N Hardcastle ${ }^{1}$, T Kron ${ }^{1}$, J Mathew ${ }^{1}$}

${ }^{1}$ Department of Physical Sciences, Peter MacCallum Cancer Centre, Australia. (Valery.Peng@petermac.org [Presenting author]), (Deloar.Hossain@petermac.org),

(Pradush.Narayanan@ @etermac.org),

(Nick.Hardcastle@petermac.org), (Tomas.Kron@petermac.org),

(Joby.Mathew@petermac.org)

Introduction Metal Oxide Semiconductor Field Transistors (MOSFETs) for in-vivo dosimetry are commonly used for verifying skin dose and dose to implanted devices. Under sufficient build-up, commercially available MOSFETs recommend a single calibration factor $(\mathrm{CF})$ for megavoltage beams. The suitability of $\mathrm{CFs}$ for a range of photon and electron energies for surface and out-of-field measurements to achieve a $5 \%$ accuracy is investigated.

Method The mobileMOSFET dosimetry system (Best Medical Canada) with dosimeters TN-502RD-H were calibrated at depth, bubble side down using various energies $(6 \mathrm{MV}, 10 \mathrm{MV}, 18 \mathrm{MV}, 6 \mathrm{E}$, 9E, 12E, 15E, 18E) from a Varian TrueBeam linear accelerator. MOSFET surface dose and out-of-field measurements (dmax) were compared to measurements from a Markus and Farmer chamber respectively using each $\mathrm{CF}$. The high sensitivity bias setting was used for out-of-field measurements, various distances outside a $10 \mathrm{~cm}$ field and the corresponding $6 \mathrm{MV}$ and $10 \mathrm{MV}$ calibrations.

Results An average photon $\mathrm{CF}$ of $1.10 \pm 0.002 \mathrm{mV} / \mathrm{cGy}$, average electron $\mathrm{CF}$ of $1.06 \pm 0.003 \mathrm{mV} / \mathrm{cGy}$ and average high sensitivity bias photon $\mathrm{CF}$ of $4.23 \pm 0.02 \mathrm{mV} / \mathrm{cGy}$ were determined, considering the standard error for uncertainty. All photon and electron measurements at dmax were within $5 \%$ of the expected dose by using the $6 \mathrm{MV}$ determined $\mathrm{CF}$. Using the electron $\mathrm{CF}$, electron surface measurements $5 \%$ of the expected dose. For photons however, dose differences of over $20 \%$ were observed regardless of CF used. For out-of-field measurements, an under response up to $10 \%$ was observed using the corresponding $\mathrm{CF}$ determined with high bias setting.

Conclusion A single $\mathrm{CF}$ can be used for measurements at dmax, while an electron-determined CF should be used for electron surface measurements. Build-up should be used for photon surface dose since their steep surface gradient does combined with an inherent MOSFET build-up does not allow for reasonable accuracy. Out-of-field measurements can be performed in high sensitivity bias mode, with a single CF but an under response is expected.

\section{O065 Commissioning of a 1.5 T MR-Linac for fully} adaptive radiation therapy

\author{
J Aylward ${ }^{1}$, K Condon ${ }^{1}$, C Pagulayan ${ }^{1}$, U Jelen ${ }^{1}$, J Arts ${ }^{2}$, \\ M G Jameson ${ }^{1,3}$
}

${ }^{1}$ GenesisCare, St Vincent's Clinic Radiation Oncology, Darlinghurst, NSW, Australia. (jack.aylward@genesiscare.com [Presenting author]), (kieran.condon@genesiscare.com),

(claire.pagulayan@genesiscare.com), (urszula.jelen@genesiscare.com). ${ }^{2}$ Elekta, North Sydney, NSW, Australia. (jason.arts@elekta.com). GenesisCare, Sydney, NSW, Australia. ${ }^{3}$ Faculty of Medicine, University of New South Wales, Australia. (michael.jameson@genesiscare.com)

Introduction Magnetic resonance image (MRI) guided radiotherapy has been gaining interest in recent years due to superior soft tissue contrast and the ability to highlight physiology [1]. There are now two types of commercial MR-Linac; the $0.35 \mathrm{~T}$ ViewRay ${ }^{\circledR}$ MRIdian and the $1.5 \mathrm{~T} \mathrm{Elekta}{ }^{\circledR}$ Unity system. Commissioning of these systems presents physicists with several challenges including, presence of magnetic field, lack of setup lasers and the requirement for nonferrous equipment and testing of MR image quality. This aim of this work is to present the procedures and results for commissioning a 1.5 T MR-Linac.

Method Following device acceptance testing, beam data collection was performed using a vendor supplied PTW ${ }^{\circledR}$ Beamscan MR water tank. Cryostat characterisation was completed with a bespoke jig, farmer chamber and a build-up cap. Other tests included MLC transmission, MV isocentre, MR-to-MV, MR image quality, beam model validation and end-to-end. Australian Clinical Dosimetry Service level $1 \mathrm{~b}$ and 3 audits were conducted.

Results All safety and mechanical tests were within vendor specification and guideline recommendations. Output variation due to cryostat attenuation was less than $2 \%$ (Figure 1). Beam model validation met AAPM TG157 [2] recommendations. All point dose measurements for end-to-end tests were within $2 \%$ and composite dose distributions over $95 \%$ passing with $3 \% / 3 \mathrm{~mm}$ global gamma criteria.

Conclusion The 1.5 T MR-Linac passed all validation and test procedures and was deemed safe for clinical use in fully adaptive radiation therapy.

\section{References}

1. Lagendijk JJ, Raaymakers BW, Raaijmakers AJ, Overweg J, Brown KJ, Kerkhof EM, van der Put RW, Hårdemark B, van Vulpen M, van der Heide UAJR, Oncology (2008) MRI/linac integration. 86 (1):25-29

2. Ma CMC, Chetty IJ, Deng J, Faddegon B, Jiang SB, Li J, Seuntjens J, Siebers JV, Traneus EJMp (2020) Beam modeling and beam model commissioning for Monte Carlo dose calculation-based radiation therapy treatment planning: Report of AAPM Task Group 157. 47 (1):e1-e18

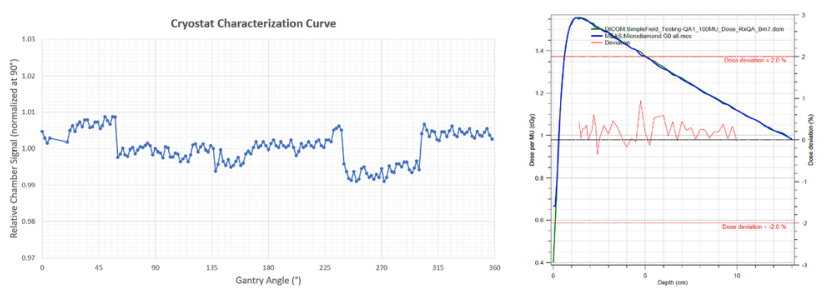

Figure 1 (left) Cryostat attenuation characterisation curve and (right) comparison of calculated and measured absolute percentage depth dose for the largest $22 \times 22 \mathrm{~cm}^{2}$ 


\section{O066 Pilot study of off-axis treatment on the inline Australian MRI-Linac system}

\section{E Patterson ${ }^{1}$, B Oborn ${ }^{1,2}$, N Roberts ${ }^{1,3}$, B Dong ${ }^{3}$, G Liney ${ }^{3}$, P Metcalfe $\mathbf{~}^{1,3}$}

${ }^{1}$ Centre of Medical Radiation Physics, University of Wollongong, Australia. (ep958@uowmail.edu.au [Presenting author]), (boborn@uow.edu.au),(nr593@uowmail.edu.au), (bin.dong@health.nsw.gov.au), (gary.liney@health.nsw.gov.au), (metcalfe@uow.edu.au). ${ }^{2}$ Illawarra Cancer Care Centre, Wollongong Hospital, NSW, Australia. ${ }^{3}$ Ingham Institute for Applied Medical Research, Sydney NSW, Australia.

Introduction MRI-Linac components produce a Lorentz force on secondary electron paths that present unique challenges for radiotherapy dosimetry [1]. Particularly for an inline MRI-Linac configuration, secondary electrons produce an enhanced, forward peaked dose along the central axis, particularly at the entry surface region. Earlier studies performed on the Australian MRI-Linac conclude that $2 \mathrm{~cm}$ of bolus placed upstream to the treatment surface is suitable to reduce electron contamination [2]. The purpose of this work was to investigate an alternative method, off axis irradiation, whereby the primary $\mathrm{x}$-ray beam is shifted laterally away from the isocentre and separates contaminant electrons from the treatment area; potentially providing superior dose uniformity [3].

Method Measurements were performed on the Australian MRI-Linac which consists of a 1T MRI (Agilent, UK) unit coupled with a $6 \mathrm{MV}$ flattening filter free (FFF) Linatron-MP (Varex, USA). Beam profiles

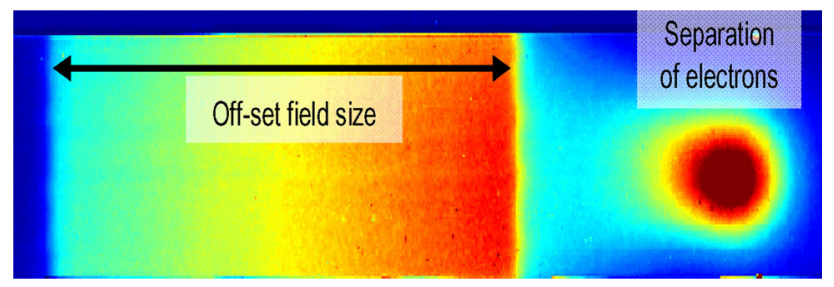

Figure $12 \mathrm{D}$ dose distribution obtained with EBT3 Film or field size of $10.1 \times 9.7 \mathrm{~cm}^{2}$ shifted $10 \mathrm{~cm}$ laterally from isocentre

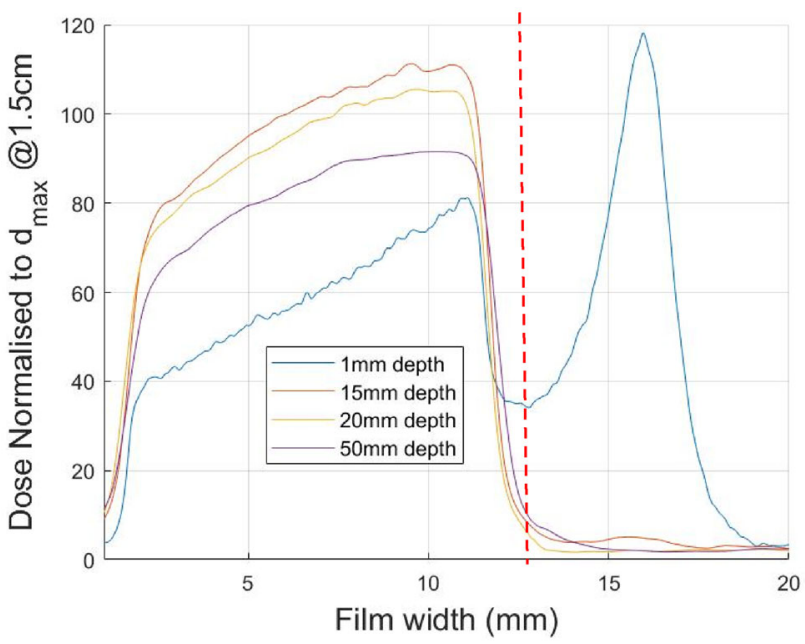

Figure 2 Dose profiles obtained with EBT3 Film for field size of $10.1 \times 9.7 \mathrm{~cm}^{2}$ shifted $10 \mathrm{~cm}$ laterally from isocentre at depths $1 \mathrm{~mm}$; $15 \mathrm{~mm} ; 20 \mathrm{~mm}$ and $50 \mathrm{~mm}$ using Gafchromic EBT3 film were irradiated perpendicular to the beam and positioned in a $30 \times 30 \mathrm{~cm}^{2}$ solid water phantom at $\mathrm{SSD}=1.8 \mathrm{~m}$. Point dose measurements were also taken with the microDiamond 60019 (PTW, Germany) and MOSkin ${ }^{\mathrm{TM}}$ detector. Three field sizes of size $2.1 \times 1.9 \mathrm{~cm}^{2}, 6.1 \times 5.8 \mathrm{~cm}^{2}$ and $10.1 \times 9.7$ $\mathrm{cm}^{2}$ were investigated and shifted approximately $10 \mathrm{~cm}$ laterally from isocentre.

Results The strong magnetic field of the MRI unit causes secondary electrons to focus at the isocentre, as shown in Figure 1. Contamination electrons result in a near surface dose of $118 \%$, relative to $d_{\max }$ as shown in Figure 2 which is readily absorbed by at least $20 \mathrm{~mm}$ of solid water. MOSkin ${ }^{\mathrm{TM}}$ measurements at $1 \mathrm{~mm}$ depth recorded $120 \%$ relative to $\mathrm{d}_{\max }$.

Conclusion By shifting the primary beam laterally from isocentre, electron contamination can be removed from the treatment beam. The clinical implication of off axis irradiation is that standard radiotherapy beams can be achieved in a high field MRI-Linac without risking excessive patient skin dose.

Acknowledgements This research has been conducted with the support of the Australian Government Research Training Program.

\section{References}

1. Oborn BM, Metcalfe PE, Butson MJ, Rosenfeld AB, Keall PJ. Electron contamination modeling and skin dose in $6 \mathrm{MV}$ longitudinal field MRIgRT: Impact of the MRI and MRI fringe field. Med Phys. (2012) 39:874-90.

2. Jelen, U., Dong, B., Begg, J., Roberts, N., Whelan, B., Keall, P. and Liney, G., 2020. Dosimetric Optimization and Commissioning of a High Field Inline MRI-Linac. Frontiers in Oncology, 10.

3. Liney GP, Dong B, Begg J et al. Technical Note: Experimental results from a prototype high-field inline MRI-linac 278 Med Phys. 2016;43:5188-5194.

\section{O067 Audit development for online adaptive radiotherapy for MRI and CBCT based systems}

R L Brown ${ }^{1}$, J Lye ${ }^{1}$, M Shaw ${ }^{1}$, R Crane ${ }^{2}$, A Briggs ${ }^{3}$, S Cooper ${ }^{2}$, B Shirley $^{2}$, A Turk ${ }^{3}$, J Lehmann ${ }^{4}$, Kate Skehan ${ }^{4}$, J Begg ${ }^{5,6,7}$, Robba Rai ${ }^{5,6,7}$, J Booth $^{3,8}$, J Baines ${ }^{2}$

${ }^{1}$ Australian Clinical Dosimetry Service, Australian Radiation Protection and Nuclear Safety Agency, Melbourne, VIC, Australia. (Rhonda.Brown@arpansa.gov.au [Presenting author]),

(Jessica.Lye@arpansa.gov.au), (Maddison.Shaw@arpansa.gov.au).

${ }^{2}$ Townsville Cancer Centre, Townsville Hospital and Health Service, Townsville, QLD, Australia. (Robert.Crane@health.qld.gov.au), (Scott.Cooper@health.qld.gov.au),

(Bronwyn.Shirley@health.qld.gov.au),

(John.Baines@health.qld.gov.au). ${ }^{3}$ Northern Sydney Cancer Centre, Royal North Shore Hospital, St Leonards, NSW, Australia.

(Adam.Briggs@health.nsw.gov.au),

(Alexandra.Turk@health.nsw.gov.au). ${ }^{4}$ Department of Radiation Oncology, Calvary Mater Newcastle, Waratah, NSW, Australia. (Joerg.Lehmann@ calvarymater.org.au),

(Kate.Skehan@calvarymater.org.au). ${ }^{5}$ Ingham Institute for Applied Medical Research, Liverpool, NSW, Australia. ${ }^{6}$ Liverpool and Macarthur Cancer Therapy Centre, Sydney, NSW, Australia. ${ }^{7}$ South Western Sydney Clinical School, University of New South Wales, Liverpool, NSW, Australia.

(Jarrad.Begg@health.nsw.gov.au), (Robba.Rai@sswahs.nsw.gov.au). ${ }^{8}$ School of Physics, University of Sydney, Camperdown, NSW, Australia. (Jeremy.Booth@ health.nsw.gov.au) 
Introduction The Australian Clinical Dosimetry Service (ACDS) has expanded its audit program to include end-to-end testing for online adaptive radiotherapy (ART) systems. Since 2019 two commercial ART systems, Elekta Unity (MR-guided RT) and Varian Ethos ${ }^{\text {TM }}$ (CBCT), became available for clinical treatment in Australia. Developing new audit cases which integrate into the existing ACDS audit structure and are consistent across both systems' imaging modalities and adaptive pathways introduces a number of challenges for phantom design.

Method New audit cases and phantom materials were implemented in the first ACDS audits performed on the Townsville Cancer Centre's Elekta Unity MRI-Linac and Royal North Shore Hospital Radiation Oncology's Varian Ethos ${ }^{\mathrm{TM}}$. Building on our existing end-to-end (Level III) audits, the 3DCRT, IMRT and SABR cases were modified and new cases were added to test the adaptive workflows. Designed for CT planning and $\mathrm{kV}$ imaging, the ACDS thorax audit phantom is opaque in MRI scans. The central solid water IMRT insert for the ACDS thorax phantom was replaced with a water-filled replica for MR image matching and dose measurement. Two new adaptive cases were introduced using a liquid soft plastic to create tissue-equivalent CT/MR-visible tumours for online imaging and also using virtual targets provided in the RT Structure set.

Results A tissue-equivalent liquid soft plastic (HU 116 \pm 14 ) provided suitable contrast against CIRS Plastic Water ${ }^{\mathrm{TM}}$ in both Unity (T1 $\mathrm{MR})$ and Ethos ${ }^{\mathrm{TM}}$ (CBCT) images. Both Unity and Ethos ${ }^{\mathrm{TM}}$ could incorporate the use of virtual targets into their adaptive workflows. Preliminary results indicate that, on both systems, the dose delivered to a concave target from a standard IMRT plan agrees with that delivered using an adaptive approach to within $1 \%$.

Conclusion The first Australian audits for online adaptive radiotherapy systems were successfully completed at the Townsville Cancer Centre and Royal North Shore Hospital Radiation Oncology, using a common phantom for MR and CT based imaging.

\section{O068 Evaluation of synthetic CTs for adaptive radiotherapy decision making}

\section{Allen ${ }^{1}$, N Hardcastle ${ }^{2}$, A Yeo ${ }^{2}$, R Franich ${ }^{1}$}

${ }^{1}$ School of Science, RMIT University, Australia. (s3655793@student.rmit.edu.au [Presenting author]. ${ }^{2}$ Department of Physical Sciences, Peter MacCallum Cancer Centre, Australia. (Nick.Hardcastle@petermac.org), (Adam.Yeo@petermac.org), (rick.franich@rmit.edu.au)

Introduction Patient anatomy varies during treatment due to physiological processes and treatment response. Cone-beam CTs (CBCTs) are acquired for patient setup but can be used to evaluate anatomical changes and plan adaptation needs. CBCTs suffer from variation in Hounsfield Units (HU) and reduced field of view, limiting dose calculation accuracy. We evaluate deformable image registration (DIR) to map HUs from the planning $\mathrm{CT}$ (pCT) to facilitate dose calculation on the CBCT for adaptive decision making.

Method For 10 head and neck cancer patients with radical intent, a replan-CT was taken within an average of 6 days of a CBCT that triggered re-planning. DIR was performed between the original pCT and the CBCT to obtain the pCT HUs with the CBCT anatomy (synthetic CT, sCT). A b-splines multi-resolution algorithm in Velocity AI software was used. The treatment plan was recalculated on the sCT retrospectively. The dose as calculated on the sCT and the replan-CT was compared using local dose-volume metrics for targets and organs-at-risk.

Results Comparisons of the sCT with other patient images are shown in Figure 1. Difference between dose metrics for the sCT and replan-
$\mathrm{CT}$ are shown in Figure 2. The mean \pm SE difference between the sCT and replan-CT metrics was $2.6 \% \pm 0.9$. The comparison showed violation of protocol metrics in 70 cases (out of total 160) for both sCTs and replan-CTs.

Conclusion We have shown that DIR has the potential to be used in the clinic for adaptive radiotherapy as a means of determining the need for re-planning to account for anatomical change (Figs. 1,2).
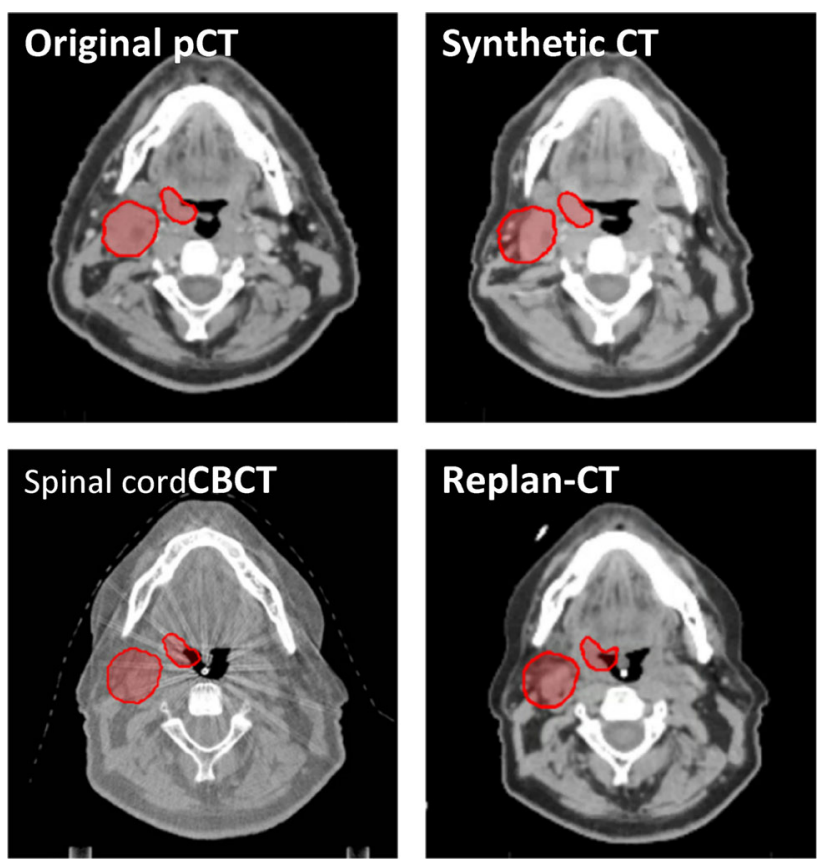

Figure 1 Axial images of a representative patient, showing the GTV (red)

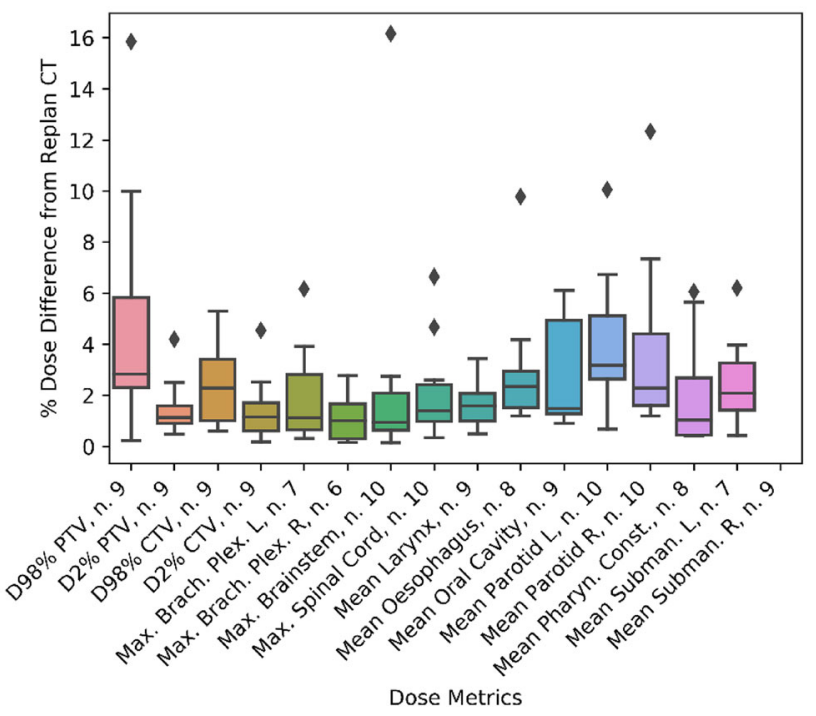

Figure 2 Calculated \%difference between metrics calculated for the replan-CTs and the sCTs. N refers to the number of contours available for analysis 


\section{Assessing the variability of MRI derived quantitative parameters used in radiotherapy treatment planning}

\section{E Carr ${ }^{1,2,3}$, P Metcalfe ${ }^{1,2}$, R Rai ${ }^{2,3,4}$, L C Holloway ${ }^{1,2,3,4,5}$}

${ }^{1}$ Centre for Medical Radiation Physics, University of Wollongong, Wollongong, Australia. ${ }^{2}$ Ingham Institute for Applied Medical Research, Liverpool, Australia. ${ }^{3}$ Department of Medical Physics, Liverpool and Macarthur Cancer Therapy Centre Liverpool, Australia. (mec640@uowmail.edu.au [Presenting author]), (metcalfe@uow.edu.au). ${ }^{4}$ South Western Sydney Clinical School, University of New South Wales, Liverpool, New South Wales, Australia. (robba.rai@ health.nsw.gov.au). ${ }^{5}$ Institute of Medical Physics, University of Sydney, Camperdown, Australia. (lois.holloway@health.nsw.gov.au)

Introduction Quantitative MRI (qMRI) has high potential in radiotherapy treatment planning (RTP), with abilities to enhance treatment response monitoring [1]. However, challenges in determining its accuracy and repeatability is limiting its clinical utility. Using standardized phantoms for such assessments has been identified as one of the keys to overcome these challenges [2]. The NIST system phantom can be used to measure qMRI relaxation times; including T1 and T2 (in ranges found within human body) [3]. This study utilised the system phantom to assess variations in MR-derived quantitative values over time.

Methods The phantom consisted of a T1 and T2 layer; each embedded with 14 spheres containing solutions with known NMR derived reference values. It was imaged on a 3T Siemens Magnetom Skyra MR-scanner. Variable Flip Angle (T1-VFA) and Inversion Recovery (T1-IR) sequences followed by nonlinear least squares fitting were used for T1 map generation. A spin-echo (multi-echo) (T2SE-ME) sequence and mono-exponential fitting were used for T2 mapping. Region of interest (ROI) statistics were then extracted from each sphere, and the coefficient of variation $(\mathrm{CoV})$ was calculated from five monthly measurements.

Results T1-IR generated less deviations from the NMR references compared to T1-VFA: Respectively, av. \%difference was $5 \%$ vs. $53 \%$. These latter measurements are known to be sensitive to field inhomogeneity's [1-4]. Further, the CoV was lowest for T1-IR $(<1 \%)$ compared to T1-VFA and T2-SE-ME methods (see Figure 1)

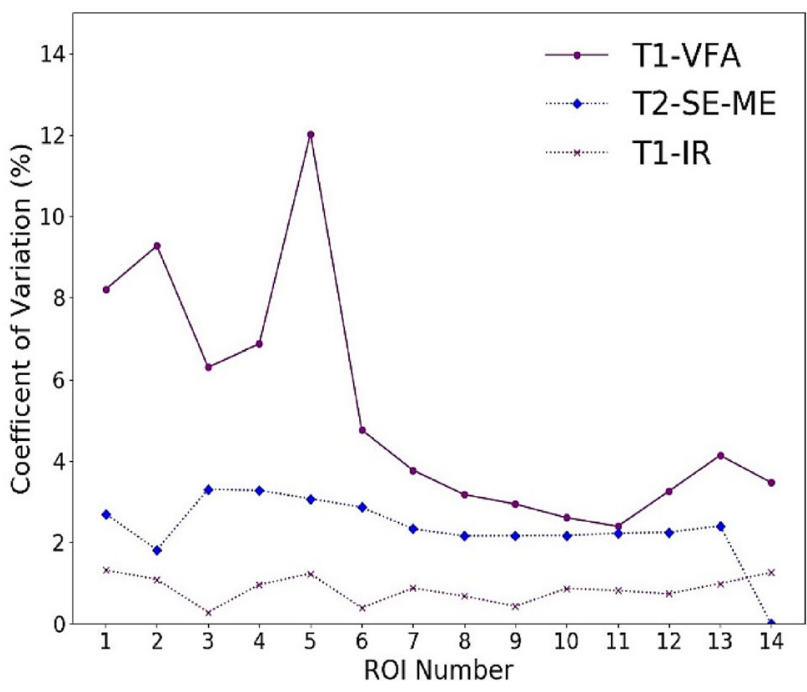

Figure 1 Repeatability of qMRI values obtained for each ROI
Conclusion Significant deviations between the measured and NMR derived $\mathrm{T} 1$ and $\mathrm{T} 2$ values were observed. Repeatability measurements were also found to fluctuate depending on the qMRI sequence utilized; T1-IR achieved the smallest variations between monthly measurements. Further longitudinal measurements are to be acquired for determination of the accuracy and repeatability of the scanner; required for effective clinical implementation of qMRI.

\section{References}

1. Bane O, Hectors SJ, Wagner M et al (2018) Accuracy, repeatability, and interplatform reproducibility of T1 quantification methods used for DCE-MRI: Results from a multicenter phantom study. Magn. Reson. Med., 79: 2564-2575. https:// doi.org/10.1002/mrm.26903

2. Shukla-Dave A, Obuchowski NA, Chenevert TL et al (2019) Quantitative Imaging Biomarkers Alliance (QIBA) recommendation for improved precision of DWI and DCE-MRI derived biomarkers in multicenter oncology trials. J. Magn. Reson. Imaging 49: e101-e121. https://doi.org/10.1002/jmri.26518

3. Russek S (2015) ISMRM/NIST MRI System Phantom. Collaborate NIST. http://collaborate.nist.gov/mriphantoms/bin/view/ MriPhantoms/MRISystemPhantoom. Accessed 20 May 2020

4. Proc.Keenan KE, Gimbutas Z, Dienstfrey A, Stupic KF (2019) Assessing effects of scanner upgrades for clinical studies. J Magn Reson Imaging, 50: 1948-1954. https://doi.org/10.1002/ jmri.26785

\section{O070 Out of field dose due to electrons generated in- field on an Elekta Unity MR linac}

\section{J Baines, M Powers}

Townsville University Hospital, QLD 4814, Australia. (john.baines@heatlth.qld.gov.au [Presenting author]), (marcus.powers@health.qld.gov.au)

Introduction During patient treatments on the Elekta Unity MR linac contaminant electrons within the primary beam and electrons produced within the patient, that enter air around the patient, spiral along the magnetic field direction $[1,2]$. In this work we present early results of investigations to evaluate these two sources of out-of-field dose. Method Following the methodology of Hackett et al [1] EBT3 film was used to determine dose due to spiralling contaminant electrons (SCE). Out-of-field dose in a B $=0 \mathrm{~T}$ environment (background dose) was compared to SCE dose in order to determine a 'background' corrected SCE dose estimate.

A phantom was used to investigate out-of-field dose arising from electrons ejected from, and produced within, the phantom by an external beam. The phantom is aligned so that electrons ejected at the beam entrance and exit spiral towards a strip of EBT3 film. Dose profiles were determined from the film to quantify the electron streaming effect (ESE) [2] at the beam entrance and exit. The attenuation of electron streams was also determined.

Finally, ESE due to beams traversing the anterior MR imaging coil was investigated.

Results Out-of-field dose with $\mathrm{B}=0 \mathrm{~T}$ is comparable to that associated with SCE, B $=1.5 \mathrm{~T}$. A background corrected SCE suggests a reduced magnitude for the SCE effect. Electron streaming from the phantom surface at the beam entrance is less than that at the exit. Electron streams are effectively absorbed by $1 \mathrm{~cm}$ bolus. The anterior coil generates electron streams depending on the field size and coil inclination.

Conclusion Doses due to the ESE are greater than SCE. Electron streams are effectively absorbed by $1 \mathrm{~cm}$ bolus. ESE from the anterior 
imaging coil can contribute to out-of-field dose. ESE and SCE should be accounted for with appropriate patient shielding..

Acknowledgements The authors acknowledge the verification of $\mathrm{B}=$ $0 \mathrm{~T}$ out-of-field dose performed by Jessica Lye, Reza Alignaghi Zadeh, Rob Behan and Sandie Fisher on the Unity MR linac at the Olivia Newton-John Cancer Wellness and Research Centre, Melbourne, Australia.

\section{References}

1. Hackett et al (2018) Spiralling contaminant electrons increase doses to surfaces outside the photon beam of an MRI-linac with a perpendicular magnetic field. Phys.Med.Biol. 095001

2. Park et al (2017) Air-stream interactions during magnetic resonance IMRT doi.org/https://doi.org/10.1007/s00066-017$1212-\mathrm{z}$

\section{O071 Experience of commissioning the HexaPOD evo RT System robotic couch top}

\section{A J Venning, M M Chandroth, B L Waller \\ Medical Physics Specialist, Mid North Coast Cancer Institute, Port Macquarie, Australia (anthony.venning@health.nsw.gov.au [Presenting author]), (Mahesh.chandroth@health.nsw.gov.au), (Brett.waller@health.nsw.gov.au)}

Introduction This abstract details the experiences learned from commissioning a HexaPOD evo RT System on an Elekta Versa HD and highlights an issue that the commissioning physicist needs to be aware of. The HexaPOD system, with 6 degrees-of-freedom is highly beneficial to treatment delivery accuracy.

Method Numerous phantoms were used to assess pitch, yaw and roll of the HexaPOD system. These included the MIMI and thorax phantoms, Rando's head and a dosimetric assessment using the ArcCHECK (1). The HexaPOD and XVI systems were separated and tested individually. For HexaPOD, known translational and rotational shifts were passed off from the XVI Registration platform to iGuide and measured using steel rulers for translations, a digital inclinometer for pitch and roll, and trigonometry for yaw rotations. For XVI, bidirectional arcs were used to image an offset phantom, and to assess that the positional error shifts were the same for each arc direction. Results In combination with the Elekta XVI kV imaging system, it was found that the HexaPOD could not achieve specification i.e. $0.2^{\circ}$ roll and sub $1 \mathrm{~mm}$ translations. However, when tested separately, by passing off known values from the XVI Registration platform, the HexaPOD performed to well within the specified tolerances. It was found that by performing bi-directional arcs, the XVI system recorded a difference in the $(\mathrm{Y})$ co-ordinate (roll) of up to $0.8^{\circ}$. This was discovered to be a function of the XVI flex maps and a small shift in the $\mathrm{kV}$ panel as it rotated.

Conclusion When commissioning a HexaPOD, it should be separated from the imaging system. In this study, it was found that the XVI coordinates provide the highest level of uncertainty in the system and need to be addressed. Before commissioning the XVI-HexaPOD combination the XVI needs to be fully characterised for rotational errors.

\section{References}

1. Chaudhari Ahmad S, Spencer K, Brahmavar S, Hyder J, Saleeby J, Bornstein L, (2012) Dosimetric Evaluation of HexaPOD as a Tool for Patient Setup Verification Accuracy Med. Phys SU-D213CD-04.

\section{O072 Medical physics commissioning of new immobilization devices across five campuses at Peter MacCallum Cancer Centre}

\author{
D Basaula ${ }^{1}$, E Ungureanu ${ }^{1}$, K Offer $^{2}, V_{\text {Peng }^{3}}, \mathrm{~J} \mathrm{Mathew}^{3}, \mathrm{~T}$ \\ Kron ${ }^{1}$ \\ ${ }^{1}$ Peter MacCallum Cancer Centre, Melbourne, Australia. \\ (deepak.basaula@petermac.org [Presenting author]), \\ (elena.ungureanu@ petermac.org). ${ }^{2}$ Peter MacCallum Cancer Centre, \\ Box Hill, Australia. (keith.offer@ petermac.org). ${ }^{3}$ Peter MacCallum \\ Cancer Centre, Bendigo, Australia. (valery.peng@petermac.org), \\ (joby.mathew@petermac.org), (tomas.kron@petermac.org)
}

Introduction Immobilisation devices are used to increase patient positioning reproducibility during radiation therapy. However such devices may result in an increased skin dose, reduced tumour dose and altered dose distribution. Our institute recently commissioned CDR Systems immobilisation devices which include several sets of the FreedomX total body immobilisation system, Sabella Flex breast immobilisation system and KOILIA MIKROS belly boards. The purpose of this study is to report the physics measurement performed for the commissioning of the new immobilisation devices prior to their clinical use.

Method Measurements using Varian Truebeam linear accelerator were performed to assess the transmission and surface dose enhancement of these immobilisation devices over a range of photon beam energies (6 MV, 6FFF, $10 \mathrm{MV}, 10 \mathrm{FFF}$ and $18 \mathrm{MV}$ ) and square field sizes. Both cylindrical (transmission) and plane parallel (surface dose) ionisation chambers were used in solid water and Perspex phantoms. An end-to-end validation testing was also performed, starting from CT simulation to treatment delivery, following the departmental clinical workflow.

Results Overall, 6 MV FFF beam energy showed the lowest transmission values for all three immobilisation systems. For the $10 \mathrm{~cm} \times$ $10 \mathrm{~cm}$ field size, transmission values of $0.96,0.97$ and 0.98 were measured for the belly board, the breast board and FreedomX total body system respectively.

Similarly, relative increase of surface dose ranges from $26 \%$ to $60 \%$ from these devices for $10 \mathrm{~cm} \times 10 \mathrm{~cm}$ field size. The largest increase resulted from the belly board system for the $6 \mathrm{MV}$ photon beam. The end to end test results were in excellent agreement with treatment planning system calculated dose (relative difference $<1 \%$ ) for VMAT type deliveries.

Conclusion The dosimetric properties of the newly purchased CDR systems immobilisation devices were characterised. It was found that these devices have been accurately modelled by the treatment planning system. Thus, these devices are deemed suitable for clinical use.

\section{O073 Characterisation of the SNC IC Profiler quad wedges for photon and electron energies}

\author{
A J Venning ${ }^{1}$, A Kovendy ${ }^{2}$ \\ ${ }^{1}$ Medical Physics Specialist, Mid North Coast Cancer Institute, Port \\ Macquarie, Australia. (anthony.venning@ health.nsw.gov.au \\ [Presenting author]), (andrew.kovendy@health.nsw.gov.au). \\ ${ }^{2}$ Director Radiation Oncology Medical Physics, Mid North Coast \\ Cancer Institute, Port Macquarie, Australia
}

Introduction This abstract details the characterisation of the SNC IC Profiler ion chamber array copper quad wedge for photons and the aluminium quad wedge for electrons for energy response [1, 2]. 
Method Measurements of the energy sensitivity of the IC Profiler with the copper quad wedge were compared against a PTW thimble $0.6 \mathrm{cc}$ chamber for the quantity $\mathrm{D} 10 \%$ in plastic water. The photon energies assessed were $6 \mathrm{MV}, 6 \mathrm{MV}$ FFF, $10 \mathrm{MV}$ FFF and $15 \mathrm{MV}$, delivered using a $30 \times 30 \mathrm{~cm}^{2}$ field size. Measurements of the energy sensitivity of the IC Profiler with the aluminium quad wedge were compared against a PTW Roos chamber for the quantity R50 in plastic water. The electron energies assessed were $6 \mathrm{MeV}, 9 \mathrm{MeV}$, $12 \mathrm{MeV}, 15 \mathrm{MeV}$ and $18 \mathrm{MeV}$, using a $25 \times 25 \mathrm{~cm}^{2}$ applicator. Measurements over multiple days on two Elekta linacs, tracked the sensitivity and magnitude of change, by exploiting the daily subtle variations in beam energy.

Results Figure 1 shows the IC Profiler with the copper quad wedge tracking against the PTW $0.6 \mathrm{cc}$ chamber for $6 \mathrm{MV}$ D10\%. Figure 2 shows the IC Profiler can track subtle photon energy changes with a comparable magnitude as a standard field chamber.

Conclusion The SNC IC Profiler with aluminium and copper quad wedges tracked subtle energy changes that are comparable with standard cross-calibrated field chambers. The tolerances applied to standard clinical ion chambers can also be applied to the IC Profiler with quad wedges. The IC profiler provides a significant QA time saving and can be used with the same confidence as standard field chambers.

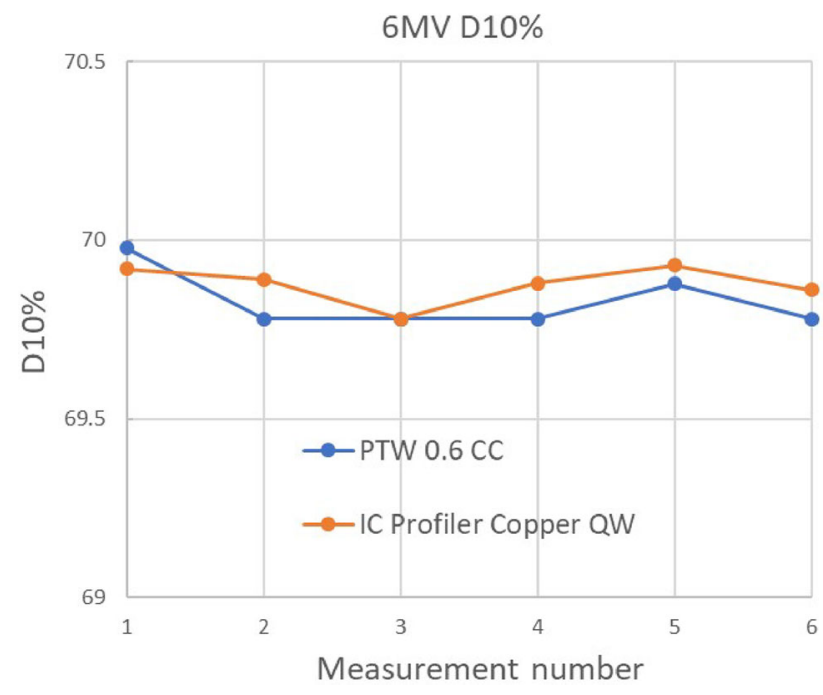

Figure 1 IC Profiler and copper wedge tracking

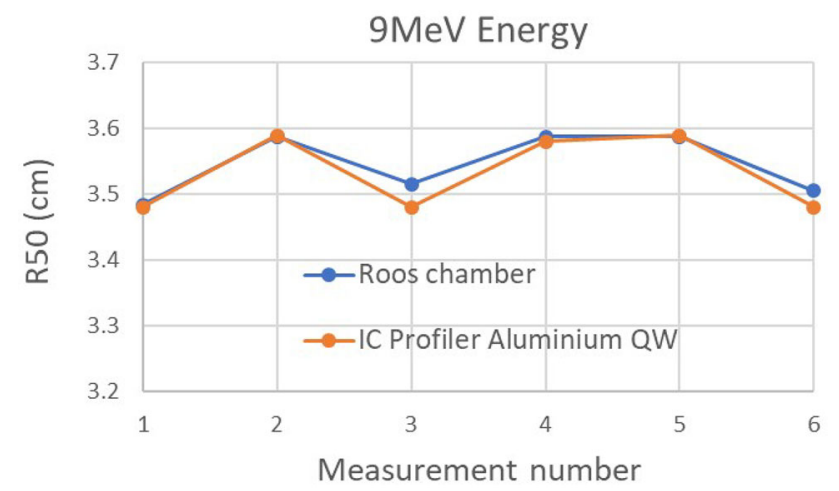

Figure 2 Response of chambers for R50

\section{References}

1. Gao S, Chetvertkov M, Simon W, Sadeghi A, Balter P (2019) Monitoring linear accelerators electron beam energy constancy with a 2D ionization chamber array and double-wedge phantom J Appl Clin Med Phys 2019; 1-8

2. Skinner L, Yang Y, Hsu A, Xing L, Yu A, Niedermayr T (2019) Factor 10 Expedience of Monthly Linac Quality Assurance via an Ion Chamber Array and Automation Scripts Technology in Cancer Research \& Treatment Volume 18: 1-10

\section{O074 A feasibility study for sub millimetre spatial resolution real time dosimetry of stereotactic radiotherapy beams}

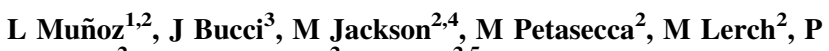 \\ Metcalfe $^{2}$, A B Rosenfeld ${ }^{2}$, G Biasi ${ }^{2,5}$ \\ ${ }^{1}$ Genesiscare Flinders Private Hospital, Bedford Park, SA, Australia. \\ ${ }^{2}$ Centre for Medical Radiation Physics, University of Wollongong, \\ NSW, Australia. (luis.munoz@genesiscare.com [Presenting author]). \\ ${ }^{3}$ St. George Cancer Care Centre, St George Hospital, Kogarah, NSW, \\ Australia. ${ }^{4}$ University of New South Wales, Kensington, NSW, \\ Australia. ${ }^{5}$ Peter MacCallum Cancer Centre, Melbourne, VIC, \\ Australia
}

Introduction Stereotactic radiosurgery (SRS) delivers small, focused beams, and its quality assurance requires measurements with high spatial and temporal resolutions [1]. The Octa is an innovative 2D dosimeter which provides temporally-resolved measurements, in real time and with a spatial resolution of 0.3 and $0.43 \mathrm{~mm}[2,3]$. This study was a first attempt at validating its use for quality assurance of SRS.

Method We considered a clinical VMAT plan to a cerebellar peduncle metastasis of size $0.422 \mathrm{cc}$, delivered as a collapsed-beam at gantry and collimator zero. We constrained the delivery because the Octa was yet to be characterised away from this configuration. Centring was completed using a square field of $5 \mathrm{~mm}$, with the Octa sandwiched between water-equivalent material at a depth of $6 \mathrm{~cm}, 94$ $\mathrm{cm}$ SSD. Measurements were benchmarked against calculations with the treatment planning system (TPS, Monaco 5.11), compared in terms of relative dose on a point by point basis, within the $80 \%$, and within the $80-20 \%$ dose level. Calculations were done on a dose grid of $1.0 \mathrm{~mm}, 1 \%$ uncertainty, but resampled on a 0.3 and $0.43 \mathrm{~mm}$ grid corresponding to the pitch of orthogonal and diagonal detector elements respectively.

Results Within the $80 \%$ level, there was a maximum percentage difference of $-2.1 \%$ in the cross-plane profile and a maximum of $2.6 \%$ in the diagonals. Within the $80-20 \%$ level, there was a maximum percentage difference of $-3.5 \%$ in the cross-plane. Cross-plane profiles with the Octa had a systematically lower intensity through the penumbra when compared to TPS (Figure 1, Figure 2). Likely attributed to multileaf collimator (MLC) characterisation i.e. leaf offset and leaf end transmission.

Conclusion This feasibility study showed, for the first time, that it is possible to ensure the quality of SRS, in real-time and with a spatial resolution of 0.3 and $0.43 \mathrm{~mm}$.

Acknowledgements We would like to GenesisCare for their continued support and access to linacs.

References

1. Wilke, L., et al., ICRU report 91 on prescribing, recording, and reporting of stereotactic treatments with small photon beams. Strahlentherapie und Onkologie, 2019. 195(3): p. 193-198. 


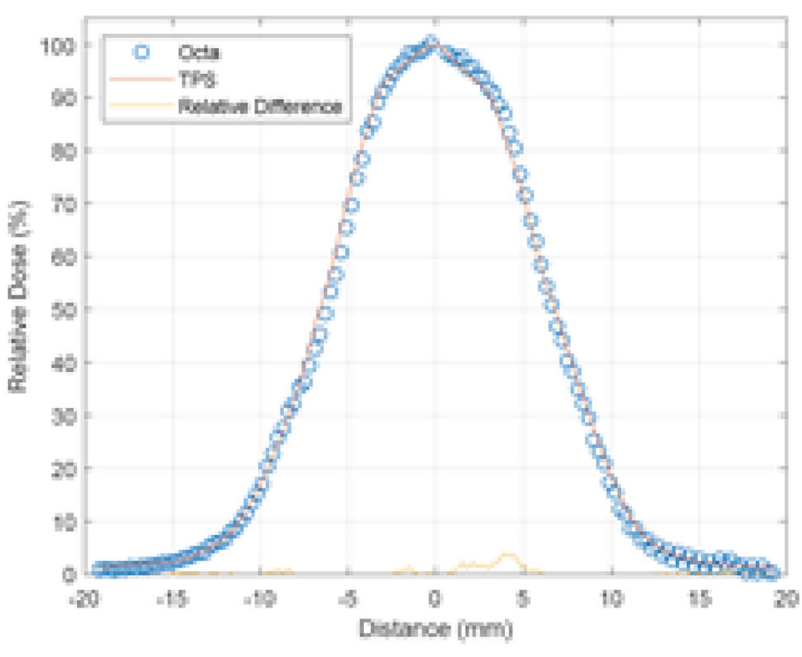

Figure 1 Cross-line profile - Octa vs TPS and relative difference

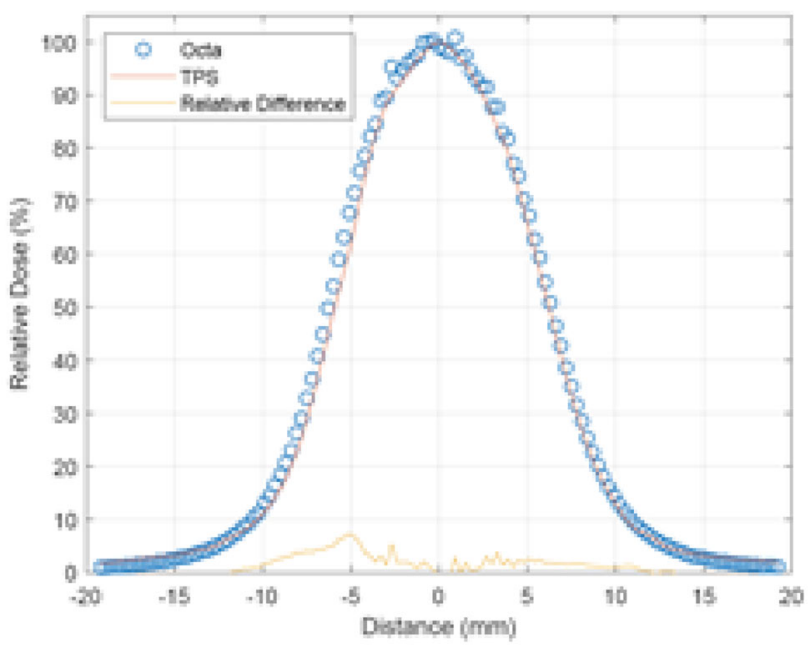

Figure 2 In-line profile - Octa vs TPS and relative difference

2. Biasi, G., et al., A novel high-resolution 2D silicon array detector for small field dosimetry with FFF photon beams. Physica Medica, 2018. 45: p. 117-126.

3. Biasi, G., et al., CyberKnife ${ }^{\circledR}$ fixed cone and Iris ${ }^{\mathrm{TM}}$ defined small radiation fields: Assessment with a high-resolution solid-state detector array. Journal of applied clinical medical physics, 2018. 19(5): p. 547-557.

\section{O075 Assessment of lung doses in patients undergoing Total Body Irradiation (TBI)}

\section{P Lonski ${ }^{1}$, M MacManus ${ }^{2}$, B A Campbell ${ }^{2}$, G Wheeler ${ }^{2}$, T Kron ${ }^{1}$}

${ }^{1}$ Department of Physical Sciences, Peter MacCallum Cancer Centre. (Peta.Lonski@petermac.org [Presenting author]),

(Tomas.Kron@petermac.org). ${ }^{2}$ Department of Radiation Oncology, Peter MacCallum Cancer Centre.

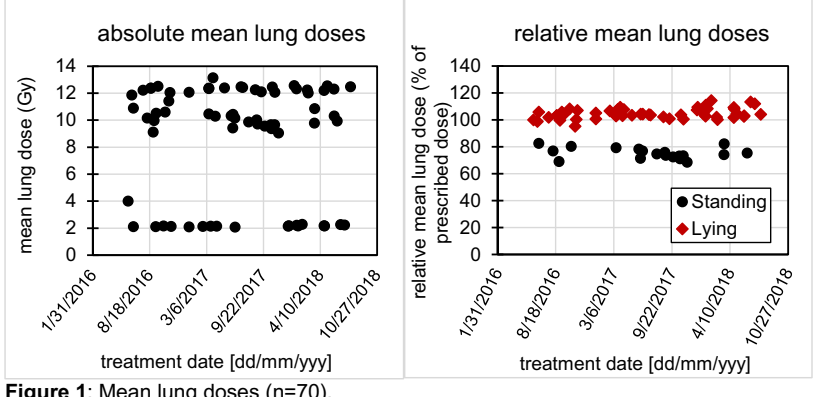

Figure 1: Mean lung doses $(n=70)$

Figure 1 Mean lung doses $(\mathrm{n}=70)$

(Michael.MacManus@petermac.org), (Belinda.Campbell@petermac.org), (Greg.Wheeler@petermac.org)

Introduction In patients undergoing Total Body Irradiation (TBI), risk factors for interstitial pneumonitis (IP) include lung dose and dose rate. However, as TBI patients are frequently treated without the aid of computer-calculated 3D radiation dose distributions, reported data on lung dose metrics in these patients is scarce. This work aims to retrospectively calculate lung dose distributions for patients who have received TBI at Peter MacCallum Cancer Centre prior to allogeneic haematopoietic cell transplantation.

Method 70 patients received TBI from 1/6/2016-31/7/2018; mean age was 38 (range 4 to 70) years. All patients were scanned using a Philips Brilliance wide bore CT scanner using $120 \mathrm{kVp}$ (paediatrics) or $140 \mathrm{kVp}$ (adults) at $2 \mathrm{~mm}$ slice thickness. 50 patients received TBI supine (prescribed 2-12 Gy in 2 Gy fractions, with chest bolus, no lung blocks) and 20 were standing (13.2 Gy in 11 fractions, with lung shields). Lungs were contoured and doses re-calculated using Eclipse v15.6 and AAA v15.606, which is commissioned and validated for TBI for 6, 10 and $18 \mathrm{MV}$ photons.

Results Mean lung doses are shown. in Figure 1. The use of lung shielding blocks for standing TBI reduced the mean lung dose to $75 \%$ of prescription dose (range, 68\%-82\%). Mean lung doses for supine TBI were an average $104 \%$ of prescribed dose (range, 95-114\%).

Conclusion Lung shielding blocks reduce mean lung doses. Supine TBI techniques may produce dose hot spots in the lungs which should be considered during treatment planning. Further work is required to associate lung dose metrics with IP rates.

References/Acknowledgements

The authors gratefully acknowledge the Peter MacCallum Cancer Centre Foundation who provided financial support for this study

\section{O076 Evaluation of treatment dose variations and dosimeter accuracy during extra corporeal bone irradiations}

\section{W Y Ip ${ }^{1}, \mathbf{R}$ Hill $^{1,2}$}

${ }^{1}$ The University of Sydney, Institute of Medical Physics, School of Physics, NSW 2006, Australia. (ipwingyan01@yahoo.com.hk [Presenting Author]). ${ }^{2}$ Department of Radiation Oncology, Chris O'Brien Lifehouse, Camperdown NSW 2050, Australia

Introduction Primary malignant bone tumours (MBT) are relatively rare when compared with other cancers but are common in children and adolescents. Extracorporeal bond irradiation (ECBI) involved delivery of high doses of radiation of up to $50 \mathrm{~Gy}$ to the bone which is taken out and re-implanted during surgery. A dependable in-vivo dosimeter would be appropriate to check the accuracy of the delivered 
dose to the bone and allow customisation of the irradiation plan. In this work, we evaluate a number of detectors for their suitability for dosimetry of ECBI.

Method The Gafchromic EBT3 film, EBT-XD film and optically stimulated luminescent dosimeters (OSLDs) were selected for investigation of the dose response up to 50 Gy. The PTW 30013 Farmer type ionisation chamber is used as a reference detector with $0.3 \%$ uncertainty. All measurements were performed on a Varian Clinac linear accelerator with $6 \mathrm{MV}$ X-ray beams. For both of the film dosimeters, post irradiation changes in optical density was evaluated. For the OSLDs, electron deep trap effects were considered as well as different annealing processes. A full ECBI experimental setup within a solid water phantom was investigated using AP/PA x-ray fields.

Results The film's result with 30 minutes fading time was also used for having a comparable standard as OSLD in time. This report had demonstrated the calibration and dose response curve in high dose for all three in-vivo detectors. OSLDs showed sub linearity curve and electron deep trap effect in high dose region. The field size will affect the measured dose in OSLD. The EBT-XD film showed better dose response than the EBT3 film for high dose radiation and able to use 30 minutes after irradiation with $2 \%$ difference.

Conclusion This report confirmed that the EBT-XD film is feasible and could be the best in-vivo detectors for ECBI.

References

1. Hong A. et al. (2001), "Extracorporeal irradiation for malignant bone tumors," Int J Radiat Oncol Biol Phys, vol. 50, no. 2, pp. $441-447$.

\section{O077 Interlace deposition: a method for 3D printing bone-like $\mathrm{HU}$ values for phantom imaging studies}

\section{R. Tino ${ }^{1,2}$, A. U. Yeo $^{3}$, M. Brandt ${ }^{1,2}$, M. Leary ${ }^{1,2}$, T. Kron ${ }^{2,3}$}

${ }^{1}$ RMIT Centre for Additive Manufacture, School of Aerospace, Mechanical and Manufacturing Engineering, RMIT University. ${ }^{2}$ ARC Training Centre in Additive Biomanufacturing, www. additivebiomanufacturing.org, Brisbane, QLD. (s3487718@student.rmit.edu.au [Presenting Author]), (milan.brandt@rmit.edu.au), (martin.leary@rmit.edu.au). ${ }^{3}$ Physical Sciences, Peter McCallum Cancer Centre, Melbourne, VIC Australia 3000. (Adam.Yeo@petermac.org), (Tomas.Kron@petermac.org)

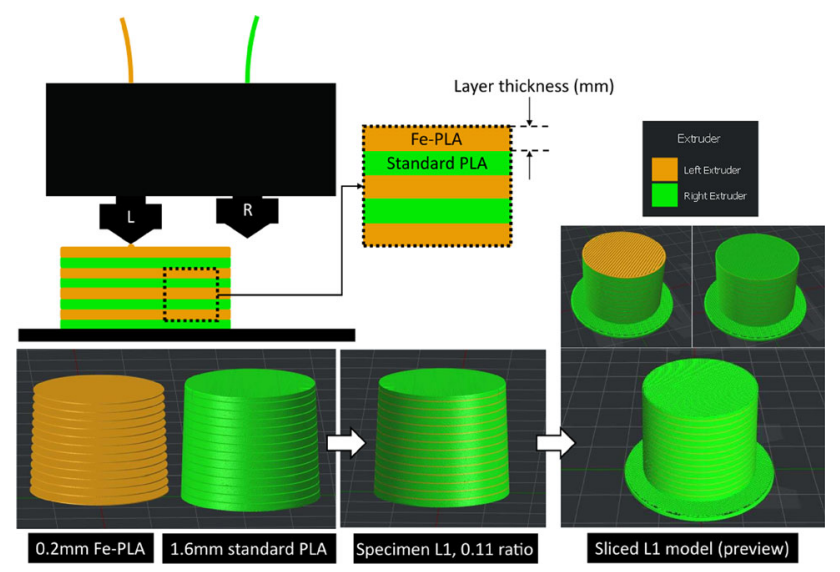

Figure 1 Illustration of interlace deposition method of standard PLA and Fe-PLA with a volume ratio of 0.11 (a layer thickness of $0.2 \mathrm{~mm}$ of Fe-PLA and a layer thickness of $1.6 \mathrm{~mm}$ of standard PLA)

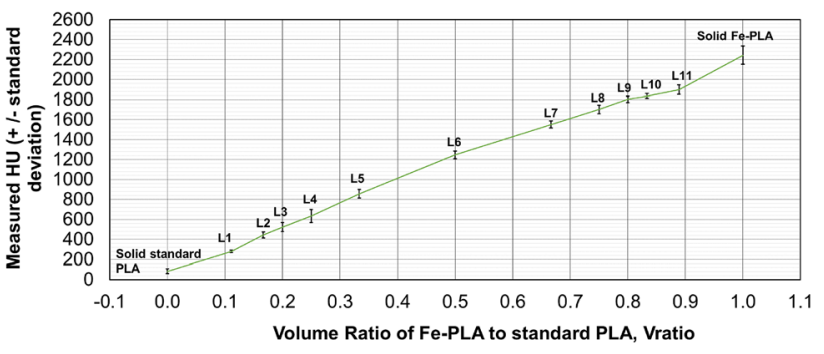

Figure $2 \mathrm{kV}$ CT results from printed specimens at $140 \mathrm{kVp}, 2 / 2$, showing material $\mathrm{V}_{\text {ratio }}$ with their associated mean $\mathrm{HU}$ value $( \pm \mathrm{SD})$

Introduction A challenge in the material extrusion printing of bonelike densities based on clinical Computed Tomography (CT) is the limited control of observed Hounsfield (HU) numbers over $0 \mathrm{HU}$ (water) when using commercially available filaments. This work developed a novel method to additively manufacture a range of densities above $1.0 \mathrm{~g} / \mathrm{cc}$ using the interlaced deposition of two commercially available filaments - standard Polylactic Acid (PLA) and iron reinforced PLA (Fe-PLA).

Method Thin PLA and Fe-PLA layers were interlaced for a total of 11 cylindrical test (L1-L11) specimens with different volume ratios, $\mathrm{V}_{\text {ratio }}$ of Fe-PLA to standard PLA ranging from 0.1 to $0.9(0=$ solid standard PLA, 1 = solid Fe-PLA), as a function of layer thickness (mm) (see Fig. 1). Standard protocol of $140 \mathrm{kVp} \mathrm{CT}$ at $2 \mathrm{~mm}$ slice thickness and increments was used for CT scanning, and mean HU and standard deviations were measured from the CT-scans.

Results The interlace deposition of cylindrical test specimens were successfully printed with minimal printing artefacts (oozing and stringing). Solid test specimens for standard PLA ( $\mathrm{V}_{\text {ratio }}$ of 0$)$ and FePLA ( $\mathrm{V}_{\text {ratio }}$ of 1$)$ were found to have mean HU values of $84 \pm 23$ and $2244 \pm 90$, respectively. Furthermore, the L1 to L11 test specimens with $\mathrm{V}_{\text {ratio }}$ ranging from 0.1 to 0.9 (Fe-PLA to standard PLA) were observed with mean $\mathrm{HU}$ values ranging from $281 \pm 12$ to $1900 \pm 46$ at $140 \mathrm{kVp}$ (2 mm slice thickness) (see Fig. 2).

Conclusion This work demonstrates a novel and effective method to additively manufacture the full range of bone-equivalent $\mathrm{HU}$ values for CT imaging studies, which have great potential for multiple applications in diagnostic imaging as well as radiation therapy.

Acknowledgements This research was conducted by the Australian Research Council Industrial Transformation Training Centre in Additive Biomanufacturing (IC160100026), www.additivebiomanufacturing.org. We further acknowledge the Peter Mac Biomedical Engineering team for their printing facility, enabling this work. Special acknowledgements to the Peter Mac Gross foundation.

\section{O078 Radiomics feature stability on 4D CT- Evaluation with 3D printed textures in motion phantom}

\author{
Hassan Albarakati ${ }^{1,2}$, Price Jackson ${ }^{1,2}$, Moshi Geso ${ }^{2}$, Rance \\ Tino $^{1,2}$, Tomas Kron ${ }^{1,2}$
}

${ }^{1}$ Department of Physical Sciences, Peter MacCallaum Cancer Centre, Melbourne, Australia. ${ }^{2}$ School of Health \& Biomedical Sciences, RMIT University, Victoria, Australia

Introduction Radiomics have shown promise for predicting outcomes in patients receiving radiotherapy for primary lung cancer. Often, however, patients are planned using motion-averaged or maximum-intensity projection reconstructions from 4D CT images which help to characterise extent of tumour motion but may degrade the appearance of highly-predictive radiomics features. In this study, 


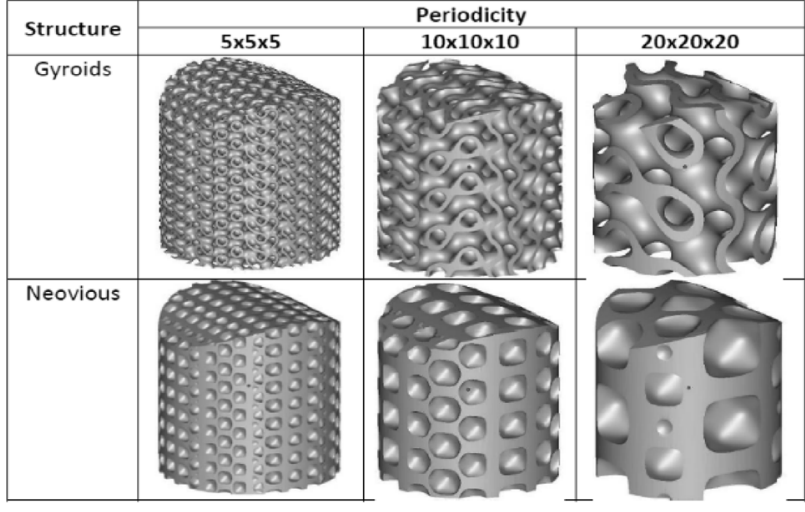

Figure 1 Views of 3D printed inserts with varying textural properties. Inserts were imaged on static (3D) and 4D CT with ranges of motion from 1 to 30 millimetres

we evaluate the stability of radiomics signal with 3D-printed inserts designed to mimic a variety of anatomically relevant tissue textures. Method 3D-printed lung inserts were created using repeating geometric structures (with modified fill repeat rate and fill factors from thermoplastic (PLA) as shown in Figure 1. CT images were acquired as static (3D) and in $4 \mathrm{D}$ with motion ranges from 1 to 30 millimetres. Static views were repeated to determine baseline feature stability by test-retest. The threshold for stability for each of the 110 features was then compared to the change in radiomics signature on MIP and average reconstructions at each scanned motion range $(1,2,5,10,20$ $\& 30 \mathrm{~mm}$ ). This was used to infer the reliable range of motion at which point any individual feature becomes unstable.

Results Of the original 110 features, 86 maintained a stable value compared to natural test-retest variability for motion ranges less than $10 \mathrm{~mm}$. Some inserts exhibited consistently better stability than others with motion. The majority of features changed from baseline sequentially with increasing motion, however nine were shown to be variable with motion indicating poor stability for $4 \mathrm{D}$ assessment. The majority of features were more reliable on maximum intensity

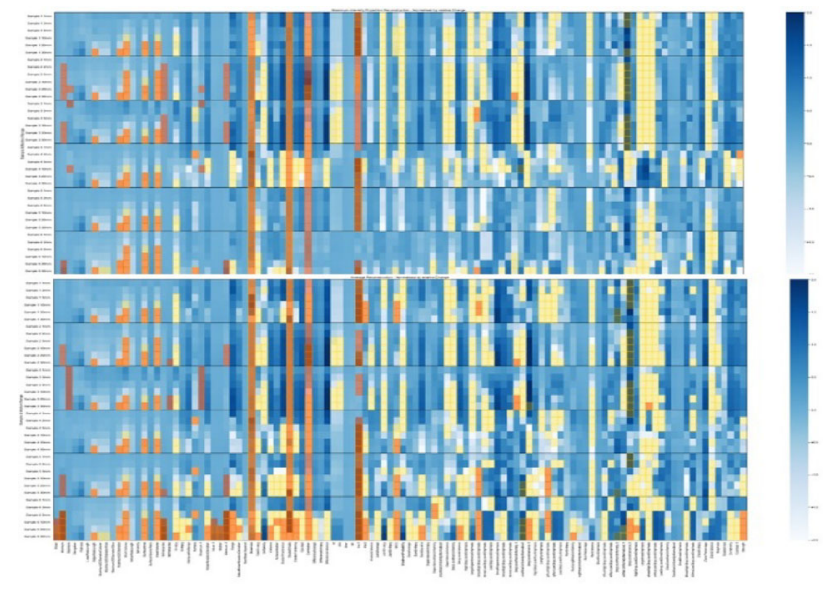

Figure 2 Percentage change from baseline for 110 evaluated radiomics features for each of 6 samples (clusters of rows) and 6 motion ranges (rows in each cluster). Changes in MIP (top) and Average (bottom) reconstructions are illustrated separately. Cells highlighted yellow change from the static image by more than the measured testretest stability. Those highlighted red change by more than the variability between all samples on $3 \mathrm{D}$ imaging projection reconstruction compared to the average reconstruction from all motion phases. An indication of degradation with motion for each insert and range is shown in Figure 2.

Conclusion This study has shown that the variability in radiomics values calculated on CT images from different motion ranges from 1 to 30 millimetres can be comparable in motion to the variability found in lung patients CT images.

\section{References}

1. Mackin, Dennis, Xenia Fave, Lifei Zhang, David Fried, Jinzhong Yang, Brian Taylor, Edgardo Rodriguez-Rivera, Cristina Dodge, A. Kyle Jones, and Laurence Court. "Measuring CT scanner variability of radiomics features." Investigative radiology 50, no. 11 (2015): 757.

2. Larue, R. T., van Timmeren, J. E., de Jong, E. E., Feliciani, G., Leijenaar, R. T., Schreurs, W. M., ... \& van Elmpt, W. (2017). Influence of gray level discretization on radiomic feature stability for different CT scanners, tube currents and slice thicknesses: a comprehensive phantom study. Acta oncologica, 56(11), 1544-1553.

3. Tanaka, S., Kadoya, N., Kajikawa, T., Matsuda, S., Dobashi, S., Takeda, K., \& Jingu, K. (2019). Investigation of thoracic fourdimensional CT-based dimension reduction technique for extracting the robust radiomic features. Physica Medica, 58, 141-148.

\section{O079 A 3D printed phantom to assess geometric distortion of MRI scanners used in a radiotherapy setting}

\section{P Ramachandran', G McGill ${ }^{2}$, M Chapman ${ }^{3}$, B Perrett ${ }^{1}$, V Seshadri $^{1}$, C Langton ${ }^{3}$}

${ }^{1}$ Radiation Oncology, Cancer Services, Princess Alexandra Hospital, Australia. (Prabhakar.Ramachandran@health.qld.gov.au [Presenting Author]), (Ben.Perrett@health.qld.gov.au),

(George.McGill@health.qld.gov.au),

(VenkataKrishnan.Seshadri@ health.qld.gov.au). ${ }^{2}$ Molecular Imaging, Biomedical Technology Services, Princess Alexandra Hospital, Australia. 33Done Australia Pty Ltd. (mike.c@3d1.com.au), (Christian.langton@3d1.com.au)

Introduction Magnetic Resonance (MR) Imaging has become a standard imaging modality for target volume delineation in radiation oncology. System-induced distortion affects geometric accuracy, and thus can influence tumor defintion and the dose delivered to the target volume. In this study, we describe a novel phantom designed to assess geometric distortion of MR images in a radiotherapy setting.

Method The phantom contains a regular grid, and the external contour of the phantom is designed so it can simulate both head \& neck and body patient geometry based on the phantom orientation. The phantom was 3D printed using an advanced selective laser sintering (SLS) 3D printing technology. A HP Jet Fusion 3D - 4200 machine was used to print the device. The phantom featured an overall length of $308.5 \mathrm{~mm}$, a width of $246 \mathrm{~mm}$ and a height of $264 \mathrm{~mm}$ including the feet and filler caps. The design also incorporates recesses inscribed on the surface of the phantom, suitable for alignment with the external laser systems available on MR simulators. The phantom can also be offset to simulate changes in geometry for different localisations of the target volume in the patient. The accuracy of the 3D print was assessed using a Toshiba Acquilion CT scanner as it has negligible geometric distortion. The phantom was scanned using 1.5T and 3T MR (Siemens) scanners for both $\mathrm{T} 1$ and $\mathrm{T} 2$ sequences to assess MR geometric distortion. 
Results The CT measurement of the grid dimensions confirmed the geometric accuracy of the 3D print. The MR phantom images for both MR scanners for T1 and T2 sequences were within tolerance in both axial and longitudinal directions and showed good agreement with the grid dimensions.

Conclusion We have designed and implemented a novel cost-effective $3 \mathrm{D}$ printed phantom suitable for assessing MR distortion in a radiotherapy setting.

\section{O080 Development of deformable phantom and its applications in quality assurance}

\section{O. Gulal ${ }^{1}$, A. U. Yeo}

${ }^{1}$ Physical Sciences, Peter McCallum Cancer Centre, Melbourne, VIC Australia 3000. (okan.gulal@ petermac.com [Presenting author]), (adam.yeo@petermac.com)

Introduction Deformable Image Registration (DIR) has become a key tool for adaptive radiotherapy to account for inter- and intrafraction organ deformation $[1,2]$. However, there is a lack of ground truth to validate DIR accuracy, particularly for low contrast regions $[3,4]$. This work aims to develop a deformable phantom which can be integrated into an anthropomorphic motion platform as a multi-purpose QA tool, so that the deformable system can be used to quantitatively and objectively assess DIR performance.

Method Platinum cured silicone gel was mixed and added into a 3Dprinted tumour mould. Two-part expanding polyurethane solution (1 Part-A, 0.45 Part-B by weight) was mixed and poured into a cylindrical mould where it expanded. Inside the mould was the siliconetumour suspended in the middle. A CIRS motion phantom was modified so that the deformable lung-rod phantom with tumour and fiducial markers (FM) could be inserted into it. Lung-rod was compressed and scanned with 4DCT (Philips Brilliance wide-bore). The FM in different breathing phases were identified and mathematically erased from the images. The intact and edited images were deformably registered. Total registration error (TRE) and Dice Similarity Coefficient (DSC) were measured.

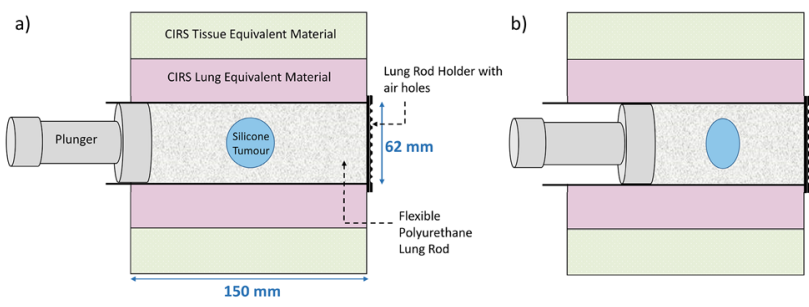

Figure 1 Lung rod phantom inside the CIRS phantom. (a) Nondeformed. (b) Deformed

Results The polyurethane-lung and silicone-tumour had a density around $0.1 \mathrm{~g} / \mathrm{cm}^{3}$ and $1 \mathrm{~g} / \mathrm{cm}^{3}$ and $\mathrm{HU}$ value around $-920 \mathrm{HU}$ and 115 HU. The lung rod phantom and silicone tumour showed elastic properties (Young's Modulus and Poisson's Ratio) in the same order of magnitude of literature [5-7]. The DSC for the tumour was above the recommend 0.8 value [2], virtual FM co-ordinates were found to have a mean TRE between $1.8 \mathrm{~mm}-5 \mathrm{~mm}$.

Conclusion This work developed a novel method to model deformable inserts using a foam and silicone phantoms in any shape, size and elasticity. Additionally, the use of fiducials and its digital erase [4] in the deformable insert could objectively measure how the algorithm performs in low-contrast areas.
Acknowledgements This research was conducted by Peter MacCallum Cancer Foundation Grants (ID1855). The authors are pleased to acknowledge helpful discussions, support, and encouragement from the Peter MacCallum Cancer Centre biomedical engineering team; Krishnan Prem, AR Anuprasan and Ssu-Yuan Nolan.

\section{References}

1. Brock, K.K., et al., Use of image registration and fusion algorithms and techniques in radiotherapy: Report of the AAPM Radiation Therapy Committee Task Group No. 132. Medical physics, 2017. 44(7): p. e43-e76.

2. Yan, D., D. Jaffray, and J. Wong, A model to accumulate fractionated dose in a deforming organ. International Journal of Radiation Oncology* Biology* Physics, 1999. 44(3): p. 665-675.

3. Yeo, U., et al., Is it sensible to "deform" dose? 3D experimental validation of dose-warping. Medical physics, 2012. 39(8): p. 5065-5072.

4. Yeo, U., et al., A novel methodology for $3 D$ deformable dosimetry. Medical physics, 2012. 39(4): p. 2203-2213.

5. Cross, S.E., et al., Nanomechanical analysis of cells from cancer patients. Nature nanotechnology, 2007. 2(12): p. 780-783.

6. Brock, K., et al., Accuracy of finite element model-based multiorgan deformable image registration. Medical physics, 2005. 32(6Part1): p. 1647-1659.

7. Fung, Y.-c., Biomechanics: mechanical properties of living tissues. 2013: Springer Science \& Business Media.

\section{O081 A comparison of CT- and photogrammetry- generated 3D prints of a HDR brachytherapy surface applicator}

\author{
C A Bridger ${ }^{1,2}$, M J J Douglass ${ }^{1,2}$, A M Caraça Santos ${ }^{1,2}$, P \\ Reich $^{1,2}$
}

${ }^{1}$ School of Physical Sciences, University of Adelaide, Australia. ${ }^{2}$ Department of Medical Physics, Royal Adelaide Hospital, Australia. (corey.bridger@adelaide.edu.au [Presenting author]),

(Michael.Douglass@adelaide.edu.au),

(Alexandre.Santos@adelaide.edu.au),(Paul.Reich@sa.gov.au)

Introduction The introduction of 3D printing in Radiation Oncology has allowed for rapid and precise design of patient-specific surface applicators using patient CT imaging data. The use of photogrammetry for generating 3D printable surface applicators has several advantages over CT imaging including reducing the amount of ionizing radiation the patient is exposed to. In this study, we investigate whether an acceptable dosimetric plan can be obtained for a surface applicator designed using photogrammetry techniques and compare the plan quality to a conventional CT derived 3D printed applicator. Method The nose region of a humanoid RANDO phantom was the selected treatment site due to its complex topology. Photographic images were captured using a Nikon D5600 DSLR camera and reconstructed using Agisoft Metashape software. CT imaging data was obtained using an Aquillion LB scanner with $1 \mathrm{~mm}$ slice thickness helical scan and $120 \mathrm{kV}_{\mathrm{p}}$ tube voltage. Virtual surface applicators with 10 catheter tunnels were designed in the software Blender and 3D printed with ABS plastic at $90 \%$ infill. Treatment plans were generated and evaluated using dosimetric parameters while their fit assessed by air gap volume measurements.

Results Both surface applicators were printed with minimal defects and visually fitted well onto the RANDO phantom. Measured air gap volume between the photogrammetry applicator and phantom surface was $44 \%$ larger than the CT applicator, indicating poorer conformity. The generated treatment plans were almost identical with a difference 
in PTV $\mathrm{V}_{100}$ of $-0.83 \%$ and skin $\mathrm{D}_{0.01 \mathrm{cc}}$ of $1.38 \%$ being the largest discrepancies for the PTV and OAR, respectively.

Conclusion A 3D printed surface applicator was successfully constructed for the RANDO phantom nose region using photogrammetry techniques. Although it produced significantly poorer conformity, its dosimetric plan was almost identical to that of the CT applicator, showing its potential for future surface applicator construction.

\section{A study of kilovoltage $x$-ray beam backscatter factors for different solid phantom materials}

\section{Robin Hill ${ }^{1,2}$}

${ }^{1}$ Department of Radiation Oncology, Chris O’Brien Lifehouse, Camperdown NSW 2050, Australia. ${ }^{2}$ The University of Sydney, Institute of Medical Physics, School of Physics, NSW 2006, Australia. (robin.hill@lh.org.au [Presenting author])

Introduction Backscatter factors (BSFs) are important dosimetric quantities for kilovoltage $(\mathrm{kV}) \mathrm{x}$-ray beams [1]. The BSF is defined as the ratio of kerma at a point located on the central axis and surface of the phantom material as compared to kerma in the material to the same point material free in air [1-2]. In this work, BSFs were calculated for a number of solid phantoms using Monte Carlo techniques for $\mathrm{x}$-ray beams with energies ranging from 30 to $150 \mathrm{kVp}$.

Method The DOSRZnrc user code of the EGSnrc Monte Carlo System V2018 (NRC, Canada) was used for the kerma calculations. A number of commonly used solid phantoms were used in the calculations. Primary $\mathrm{x}$-ray beam spectra were calculated using the SpekCalc code. BSFs were calculated for each phantom with field sizes from $2-20 \mathrm{~cm}$ diameter. The total number of incident photons for each calculation was such that type-A uncertainties were less than $0.5 \%$.

Results The BSFs for RMI457 Solid Water, Virtual Water and Plastic Water DT were generally within $1.0 \%$ compared to those calculated in water. In comparison, the BSF for Plastic Water was up to 5\% less than those calculated in water with a maximum difference at $100 \mathrm{kVp}$. For both PMMA and polystyrene, the BSF were up to $9 \%$ greater than those calculated in water particularly for the lower energy x-ray beams. However BSFs for polystyrene were less than those in water at the higher energies. Conclusion Some phantoms have BSFs in very good agreement to those in water being RMI457 Solid Water, Plastic Water DT and Virtual Water. In comparison, differences of up $9 \%$ were found with polystyrene as compared to water. Therefore careful selection is recommended in using solid phantoms for the dosimetry of kilovoltage x-ray beams.

\section{References}

1. Hill, R., et al. (2014), Advances in kilovoltage x-ray beam dosimetry. Phys Med Biol, 59(6): p. R183.

2. Andreo, P. (2019). Data for the dosimetry of low-and mediumenergy kV x rays. Phys Med Biol, 64(20), 205019.

3. Poludniowski, G., et al. (2009), SpekCalc: a program to calculate photon spectra from tungsten anode x-ray tubes. Phys Med Biol, 54(19): p. N43.

\section{A method of assessing and correcting for temporal changes in optical density using radiochromic film: Can we get instant results?}

\section{F Dunn}

GenesisCare, Centre for Radiation Oncology, St Vincent's Hospital, Fitzroy, VIC. (leon.dunn@genesiscare.com [Presenting author])
Introduction The aim of this work was to create an automated workflow to allow radiochromic film (EBT3 / XD) to be used as an almost instantaneous dosimeter.

Method Ten GafChromic EBT3 calibration films were exposed in 2 Gy increments up to $20 \mathrm{~Gy}$. Calibrates were then scanned at fiveminute intervals post exposure over 24 hours using an AutoHotKey script resulting in 282 tiff images. Following the 24-hour scanning period, a MATLAB script was used to automatically read in the tiff images and create a series of 282 calibration curves distinct in time which is termed the "temporal calibration model" (TCM). The model is saved as a series of polynomial fit coefficients to net optical density as a function of dose, timestamped in five-minute increments. Five patient-specific film measurements were then carried out and scanned using the same five-minute scan intervals from five-minutes post exposure to 24 hours post exposure. The TCM was then automatically

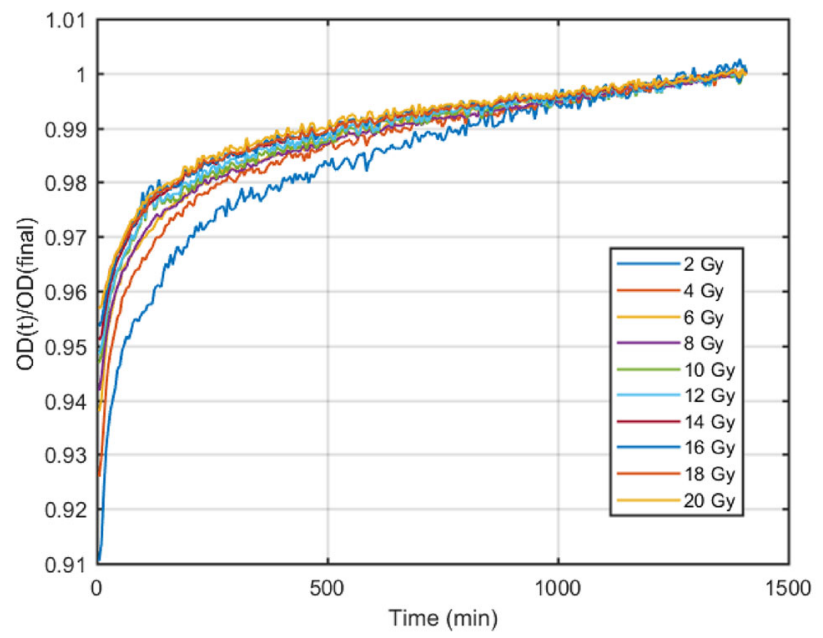

Figure 1 Net optical density changes per dose level as a function of time normalised to the net optical density of the calibrate at 24 hours post exposure (resolution $=5$ minutes)

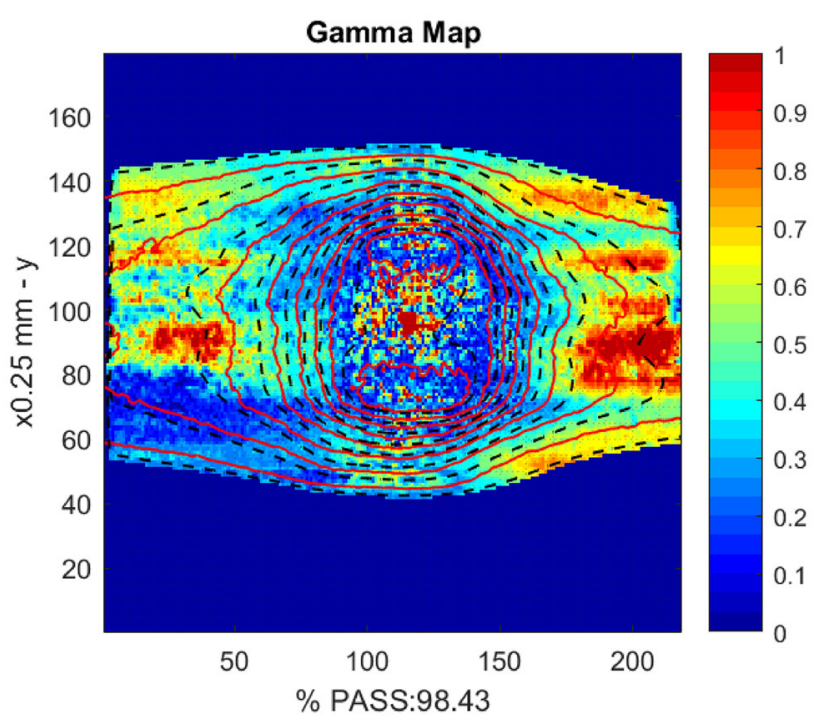

Figure 2 Gamma map comparison ( $2 \% / 2 \mathrm{~mm}$ criteria) for a patientspecific QA measurement using EBT3 film (10 Gy max) scanned at $\sim 5$ minutes post exposure and corrected using the TCM 
applied using in-house software eFilmQA to convert the patientspecific QA films to dose based on applying the relevant calibration curve from the TCM corresponding to the arbitrary time post exposure that the film was scanned. Each dose plane at post-exposure scan intervals of 5, 10, 15, 20, 30 minutes and 24 hours was then compared to the treatment planning system dose prediction using gamma analysis.

Results The TCM accurately converted the film scan optical density to dose irrespective of the time of scanning post-exposure. Gamma pass rates normalised to the pass-rate after 24 hours post-exposure were found to be within $3 \%$ (3\%/3 mm criteria) for all patientspecific QA measurements.

Conclusion We have developed a methodology that allows for radiochromic film to be accurately used as a dosimeter at any arbitrary scan time post-exposure, where previously a waiting period of $15-$ $24 \mathrm{hrs}$ before readout was needed to ensure the post exposure development had stabilised. The creation of a TCM can therefore enable results from radiochromic film measurements to be obtaine$\mathrm{d}$ as quickly as needed (Figs. 1, 2).

\section{O084 Cranial SRS phantom analytical methods used in dosimetry audits for complex treatments of multiple brain metastases.}

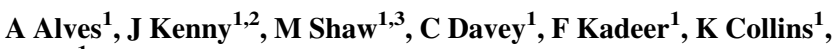 \\ J Lye ${ }^{1}$ \\ ${ }^{1}$ Australian Clinical Dosimetry Service, ARPANSA, Melbourne, \\ Australia. (andrew.alves@arpansa.gov.au), \\ (maddison.shaw@arpansa.gov.au), (cate.davey@arpansa.gov.au), \\ (fayz.kadeer@arpansa.gov.au), (katherine.collins@arpansa.gov.au), \\ (jessica.lye@arpansa.gov.au). ${ }^{2}$ Health STEM Solutions, Melbourne, \\ Australia. (john.kenny@arpansa.gov.au [Presenting author]). ${ }^{3}$ RMIT \\ University, Melbourne, Australia
}

Introduction We report the analytical methods used in assessing data from the ACDS end-to-end stereotactic radiosurgery (SRS) audit. The audit includes: MR imaging; small distal and dispersed targets; single or multiple isocentres; compatibility with various treatment platforms. Assessments must enable dosimetry credentialing in the TROG Local HER-O [1] and OUTRUN [2] trials, chiefly using film to report on the treatment's spatial accuracy, and diamond detector and film for dosimetry.

Method Facilities apply their preferred technique and clinical protocols in three audit cases and two audit cases have stationary beams. GTV/PTV and OAR dose constraints are defined. Targets are either delineated from facility MR images, of provided via DICOM RT structure set. An IMT MAX-HD ${ }^{\mathrm{TM}}$ (IMT, New York) cranial
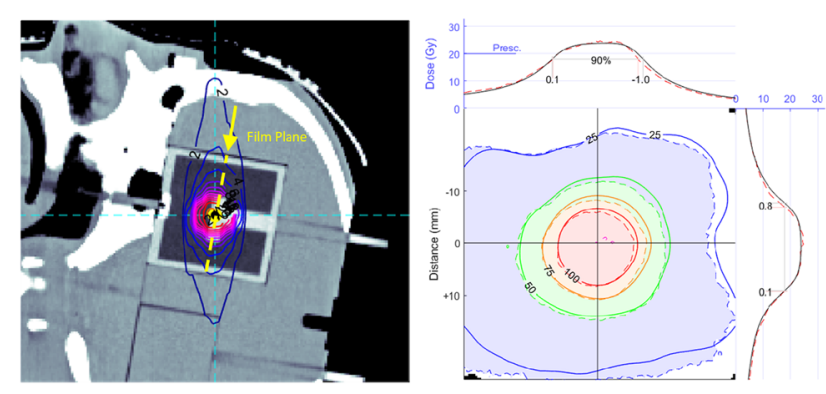

Figure 1 A film plane is tilted with respect to the axes of the DICOM data. Film vs planned dose comparison after extraction of the dose data from the film plane phantom with tissue equivalent materials and realistic bony anatomy suitable for IGRT is used. The phantom has CT/MR fiducial markers and cavities for dosimetry measurements. Dosimetry is performed using PTW microdiamond detectors (PTW Freiburg, Germany) and Gafchromic EBT3 and XD film (Ashland Inc., Bridgewater NJ, USA). Clinical immobilization must be used to hold the phantom. The resulting film planes in the phantom are often not congruent with $3 \mathrm{D}$ axes of the DICOM data.

Results Measurements have been conducted at six sites to-date. Point dose variations by microdiamond measurements range over approx. $\pm 10 \%$. Phantom film planes are tilted by up to 10 degrees with respect to the DICOM x-y-z planes. MatLab code has been developed to extract 2D dose maps at any tilt angle to allow assessment of the treatment at the film locations (Fig. 58).

Conclusion Tilted dose plane extraction has enabled distance-toagreement and 2D gamma analysis metrics which are now being reported.

\section{References/Acknowledgements}

1. Australian Cancer Trials Registry: Sydney (NSW): NHMRC Clinical Trials Centre, University of Sydney (Australia); 2005 - . Identifier ACTRN12616001265460. TROG 16.02 Local HER-O A study of local therapy for the treatment of brain metastases from Human Epidermal Growth Factor Receptor Type 2 (HER2) positive breast cancer 2016 Sep 8. http://anzctr.org.au/ACTRN1261600 1265460.aspx

2. ClinicalTrials.gov [Internet]: Bethesda (MD): National Library of Medicine (US) 2016 Jul 14 - . Identifier NCT03497767, TROG 17.02 Randomised phase II trial of Osimertinib with or without stereotactic radiosurgery for EGFR mutated NSCLC with brain metastases. 2016 Sep 8. https://clinicaltrials.gov/ct2/show/ NCT03497767

The ACDS would like to acknowledge:

TROG Cancer Research for use of the TROG planning competition OAR structure set in the audit.

The facilities who have performed the audit.

\section{O085 Over-response of EBT3 film in lung phantoms irradiated small fields}

\author{
PH Charles ${ }^{1,2}$, T Kairn ${ }^{2,3}$, S B Crowe $\mathrm{C}^{1,2,3}$
}

${ }^{1}$ Herston Biofabrication Institute, Brisbane, Australia. ${ }^{2}$ Science and Engineering Faculty, Queensland University of Technology, Australia. (Paul.Charles@health.qld.gov.au [Presenting author]), (Tanya.Kairn@health.qld.gov.au), (Scott.Crowe@health.qld.gov.au). ${ }^{3}$ Radiation Oncology, Royal Brisbane and Women's Hospital, Australia.

Introduction Detectors used for small field dosimetry can exhibit field size dependent sensitivity [1]. It has been previously shown that EBT3 film requires no correction factor for small field dosimetry measurements in water due to its similar density [2, 3]. However to date, no studies have confirmed the suitability of EBT3 film for the conditions of extreme electronic disequilibrium presented by small fields measured within lung equivalent phantoms. This study used Monte Carlo simulations to evaluate EBT3 film in lung phantoms. Method All simulations were performed in DOSXYZnrc [4]. The phantom simulated was $20 \mathrm{~cm}$ in each dimension. In the beam direction it was layered $5 \mathrm{~cm}$ of water, $10 \mathrm{~cm}$ of lung (density of 0.26 $\mathrm{g} / \mathrm{cm}^{3}$ ) and $5 \mathrm{~cm}$ water - with each layer extending to the phantom edge. All simulations were performed with a $6 \mathrm{MV}$ photon beam from a Varian iX linear accelerator for square fields sizes of side lengths from $10 \mathrm{~mm}$ to $30 \mathrm{~mm}$. 
Table 1 Central axis correction factors

\begin{tabular}{llll}
\hline Field size $(\mathrm{mm})$ & \multicolumn{2}{l}{ EBT3 film correction factor } \\
\cline { 2 - 4 } & $\begin{array}{l}\text { Central } \\
\text { axis }\end{array}$ & $\begin{array}{l}\text { Upstream } \\
\text { interface }\end{array}$ & $\begin{array}{l}\text { Downstream } \\
\text { interface }\end{array}$ \\
\hline 10 & 0.953 & 0.982 & 0.966 \\
15 & 0.979 & & \\
20 & 0.996 & & \\
30 & 1.000 & & \\
\hline
\end{tabular}

EBT3 film was simulated at various planes in the phantom (film perpendicular to beam direction), including at the centre of the lung and at the upstream and downstream interfaces between the lung medium and the water. For each film simulated, an identical simulation was performed where the film was replaced with lung. The correction factor required for film was calculated by dividing the simulation results of the lung phantom without film by the results for the film at each position.

Results EBT3 was found to over-respond by 2 to $5 \%$ when the fields were very small [5]. Table 1 displays the central-axis correction factors required to account for this over-response. The uncertainty in each result is $\sim 0.5 \%$.

Conclusion EBT3 film is an acceptable dosimeter to use without correction factors for $6 \mathrm{MV}$ field sizes $20 \times 20 \mathrm{~mm}^{2}$ and larger. For smaller fields sizes, care should be taken to correct the over-response of EBT3 film in lung phantoms.

References/Acknowledgements

1. Dosimetry of Small Static Fields Used in External Beam Radiotherapy (2017). TRS483. INTERNATIONAL ATOMIC ENERGY AGENCY, Vienna

2. Charles P (2019) Small field dosimetry using film in plastic phantoms - a Monte Carlo study Australasian Physical \& Engineering Sciences in Medicine 42 (1):285-401. https://doi.org/ 10.1007/s13246-019-00724-x

3. Morales JE, Butson M, Hill R, Crowe SB, Trapp JV (2020) Monte Carlo calculated output correction factors for Gafchromic EBT3 film for relative dosimetry in small stereotactic radiosurgery fields. Phys Eng Sci Med 43 (2):609-616. https://doi.org/ 10.1007/s13246-020-00860-9

4. Kawrakow I (2015) The EGSnrc code system. PIRS701 https:// nrc-cnrc.github.io/EGSnrc/doc/pirs701-egsnrc.pdf

5. Charles PH, Cranmer-Sargison G, Thwaites DI, Crowe SB, Kairn T, Knight RT, Kenny J, Langton CM, Trapp JV (2014) A practical and theoretical definition of very small field size for radiotherapy output factor measurements. Med Phys 41 (4):041707. https://doi.org/10.1118/1.4868461

\section{O086 Investigation of target dose conformity using normoxic polymer gel dosimetry techniques: a clinical example of 12th thoracic vertebrae SBRT treatment with VMAT}

\section{A J Venning, M M Chandroth, C Morgan, M Roberts}

Medical Physics Specialist, Mid North Coast Cancer Institute, Port Macquarie, Australia. (anthony.venning@health.nsw.gov.au [Presenting author]). (Mahesh.chandroth@health.nsw.gov.au),

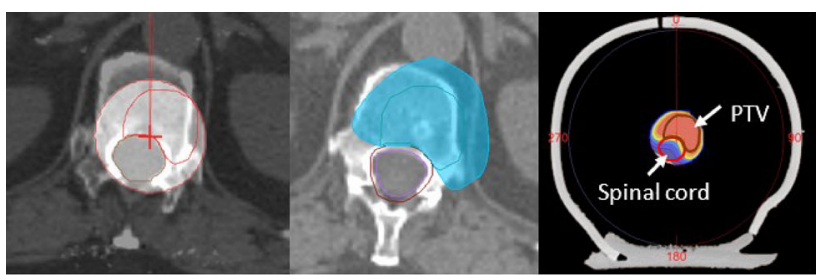

Figure 1 Thoracic 12 fused with gel vial

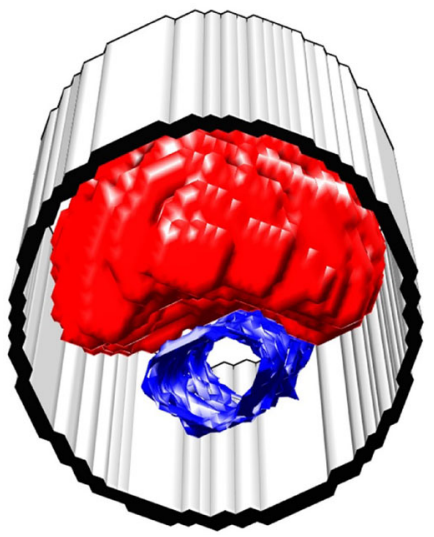

Figure 25 Gy isodose sphere (red), spinal cord (blue) inside gel filled vial

(Cian.morgan@healthcareimaging.com.au), (Murray.roberts@healthcareimaging.com.au)

Introduction Using 3D normoxic polymer gel dosimetry techniques with MRI readout (1), this study presents a 3D dosimetric analysis of a 12th thoracic vertebrae (T12) VMAT SBRT treatment.

Method Gel dosimeters were manufactured using materials and methods described elsewhere [1], poured into a $47 \mathrm{~mm} \times 150 \mathrm{~mm}$ vial, simulating the spine and immersed within a lung ED tissue equivalent, water-soaked sponge wrapped in Superflab, simulating chest wall. CT images of the gel-phantom were exported to the TPS. A patient's bi-arc VMAT plan was copied onto the CT data set and recalculated retaining MLC beam shapes, creating a curved dose distribution around the T12 spinal canal (Fig. 1). The phantom with dosimeter was irradiated according to the plan and imaged using a $3 \mathrm{~T}$ MRI with $T_{2}$ maps generated [2]. A 3D dose matrix was created from the individual dose maps and the $5 \mathrm{~Gy}$ isodose line of interest was plotted in 3D in MATLAB ${ }^{\mathrm{TM}}$. The patient's CT dataset was imported into MATLAB ${ }^{\mathrm{TM}}$ and the spinal cord contour surrounded by the T12 vertebra was extracted as per Fig. 1. From the fused images, the position of the vial that encompassed both the PTV and spinal cord contours was established. The key assessment criteria for this study was to observe if the $5 \mathrm{~Gy}$ isodose line was shaped around the spinal cord and the delivery did not violate the isodose constraint.

Results Figure 2 shows the 5 Gy (red) isodose sphere of interest wrapped around the spinal cord (blue). At no point does the 5 Gy isodose line violate the spinal cord isodose constraint.

Conclusion This study found that the full delivery chain, from end-toend can safely deliver a high dose fractionation regime to complex and tight margins, resulting in improved patient outcomes.

References 
1. Venning AJ, Hill B, Brindha S, Healy BJ, Baldock C (2005) Investigation of the PAGAT polymer gel dosimeter using magnetic resonance imaging Phys. Med. Biol. 50 3875-88

2. De Deene Y and Baldock C (2002) Optimization of multiple spin-echo sequences for 3D polymer gel dosimetry Phys. Med. Biol. 47 3117-41

\section{O087 Further investigation of lung tumour peripheral doses using normoxic polymer gel dosimetry techniques with a range of MV photon energies}

\section{A J Venning, M M Chandroth, C Morgan, M Roberts}

Mid North Coast Cancer Institute, Port Macquarie, Australia. (anthony.venning@health.nsw.gov.au [Presenting author]), (Mahesh.chandroth@health.nsw.gov.au),

(Cian.morgan@healthcareimaging.com.au),

(Murray.roberts@healthcareimaging.com.au)

Introduction This study improves upon a previous investigation of lung tumour peripheral doses using a novel in-house manufactured lung-tissue simulator $[1,2]$.

Method To simulate lung tumour, foam material was cut and shaped into the size of a human lung and wrapped with $1 \mathrm{~cm}$ thick Superflab bolus simulating chest wall. A $47 \mathrm{~mm}$ diameter hole was drilled into the centre of the sponge where a gelatine filled PET vial was placed. The foam was saturated in water to be equivalent to that of average lung tissue electron density ( $\sim$ RED 0.250 ). The phantom was CT scanned and images sent to the Monaco ${ }^{\circledR}$ TPS for planning.

Conformal arc plans were generated for $6 \mathrm{MV}, 6 \mathrm{MV}$ FFF and $10 \mathrm{MV}$ FFF, with field size $4.8 \mathrm{~cm}^{2}$ and $450 \mathrm{MU}$. The central axis transverse dose planes were exported for comparison with measurements.

Gel dosimeters were manufactured using materials and methods described elsewhere [3], poured into vials and immersed within the sponge. The sponge with dosimeters were irradiated according to the treatment plan and subsequently imaged using a 3T MRI with $T_{2}$ maps generated [4]. Profile comparisons between the TPS and dosimeters were performed using Matlab ${ }^{\mathrm{TM}}$.

Results Measurements show that Monaco ${ }^{\circledR}$ over estimates dose in the build-up peripheral region of tumour equivalent tissue in lung for the

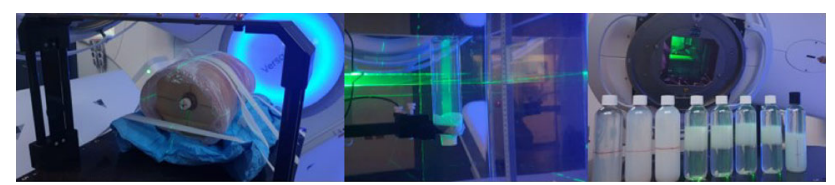

Figure 1 Irradiation of gelatine filled vials
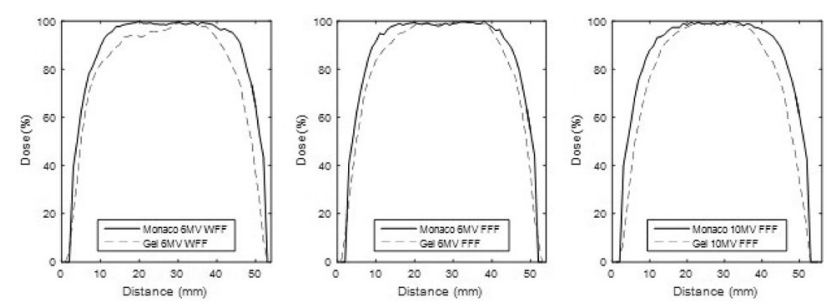

Figure 2 Profiles showing an overestimation of Monaco ${ }^{\circledR}$ photon energies investigated, as per Figure 2. These results are similar to results from an earlier version of this study [1] (Fig. 61).

\section{References}

1. Venning A J, Chandroth M, Chick B J, Waller B and Morgan C 2019 Investigation of lung tumour peripheral doses using normoxic polymer gel and film dosimetry techniques $J$. Phys. Conf. Ser. 1305 (2019) 012003

2. Chandroth M, Venning A J, Chick B J, Waller B L, Optimum photon beam procurement in modern radiotherapy departments 2019 ESTRO meets Asia S79 PO-177

3. Venning AJ, Hill B, Brindha S, Healy BJ, Baldock C (2005) Investigation of the PAGAT polymer gel dosimeter using magnetic resonance imaging Phys. Med. Biol. 50 3875-88

4. De Deene Y and Baldock C (2002) Optimization of multiple spin-echo sequences for 3D polymer gel dosimetry Phys. Med. Biol. 47 3117-41

\section{O088 Commissioning of a Magnettech MS5000 electron paramagnetic resonance spectrometer for radiation dosimetry}

\section{Christopher Noble, Prabhakar Ramachandran}

Radiation Oncology, Princess Alexandra Hospital, Ipswich Rd, Brisbane, Australia. (Christopher.Noble@ health.qld.gov.au [Presenting author]), (Prabhakar.Ramachandran@health.qld.gov.au)

Introduction In the 1960s it was observed that irradiated alanine had a long lived radical that could be easily measured with EPR spectroscopy and the alanine/electron paramagnetic resonance (EPR) system was proposed as a tool for radiation dosimetry [1]. Currently, NIST in the US [2] the NPL in the UK [3], and the Helmholtz Institute in Germany [4] have implemented an alanine/EPR dosimetry service. The Princess Alexandra Hospital recently purchased a Magnettech MS5000 EPR spectrometer as a research tool with the aim of developing protocols for EPR based in-vivo dosimetry for use in radiotherapy.

Method A protocol for commissioning EPR spectrometers for radiation dosimetry was developed. Standard tests were performed that included, temperature and energy dependence, dose linearity, and reproducibility. Measurements were made using a calibration set of dosimeters that were irradiated with $2 \mathrm{MeV}$ electrons by the manufacturer and with dosimeter pellets irradiated at the energies used clinically in the department. In addition, tests were made to control for possible sources of magnetic interference from linear accelerators in adjacent bunkers.
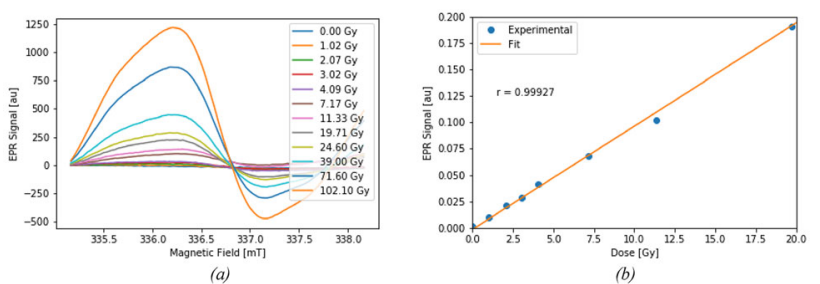

Figure 1 Calibration curve for alanine dosimeters a) The peak-topeak amplitude of the EPR spectrum increases with the absorbed dose b) The EPR signal is linear with dose 
Results Figure 1 show the EPR spectrum for the calibration dosimeter set with $2 \mathrm{MeV}$ irradiation from 0 to $102 \mathrm{~Gy}$. The EPR signal strength was linear with dose over the measured range.

Conclusion The dose linearity and energy dependence are both suitable for dosimetry in a radiotherapy setting. As expected, a strong dependence of the EPR signal on measurement temperature was observed which can be controlled for by allowing the spectrometer to reach equilibrium before measurement. The largest source of error was in sample positioning which needs to be addressed in order to achieve the accuracy necessary for in-vivo dosimetry.

\section{References}

1. W. W. Bradshaw, D. G. Cadena, G. W. Crawford, and H. A. W. Spetzler, "The Use of Alanine as a Solid Dosimeter The Use of Alanine as a Solid Dosimeter'," Radiat. Res., vol. 17, no. 17, pp. 11-21, 1962, Accessed: Jul. 04, 2018. [Online]. Available: http://www.jstor.org/stable/3571206.

2. D. F. Regulla and U. Deffner, "Dosimetry by ESR spectroscopy of alanine," Int. J. Appl. Radiat. Isot., 1982, https://doi.org/ 10.1016/0020-708x(82)90238-1.

3. P. H. G. Sharpe and J. P. Sephton, "Alanine dosimetry at NPL the development of a mailed reference dosimetry service at radiotherapy dose levels," in IAEA-SM-356/R6 Proc., 1998, pp. 183-189, Accessed: Jul. 04, 2018. [Online]. Available: http:// www.iaea.org/inis/collection/NCLCollectionStore/_Public/30/ 019/30019778.pdf.

4. M. F. Desrosiers and J. M. Puhl, "Absorbed-dose/dose-rate dependence studies for the alanine-EPR dosimetry system," Radiat. Phys. Chem., vol. 78, no. 7-8, pp. 461-463, 2009, https:// doi.org/10.1016/j.radphyschem.2009.03.025.

\section{Plasma therapy for cancer: a new approach for delivery of active agents without radiation beams}

\author{
J Harley $^{1}$, N Suchowerska ${ }^{2}$, D R McKenzie ${ }^{1,2}$ \\ ${ }^{1}$ The University of Sydney, School of Physics, Australia \\ and VectorLAB, Chris O'Brien Lifehouse, Sydney, Australia. \\ (jhar9271@uni.sydney.edu.au [Presenting author]). ${ }^{2}$ VectorLAB, \\ Chris O'Brien Lifehouse, Sydney, Australia and The University \\ of Sydney, School of Physics, Australia. \\ (Natalka.Suchowerska@lh.org.au), (david.mckenzie@sydney.edu.au)
}

Introduction Reactive oxygen and nitrogen species (RONS) are active agents in radiation therapy, responsible for a large fraction of the cancer cell death. However in conventional radiotherapy, external beams deliver an entrance and exit dose to normal tissue, while the use of radioactive isotopes in brachytherapy has inherent handling risks. Cold atmospheric plasma has been shown to generate RONS when directly applied to cells in culture and when applied to the culture medium itself, potentially enabling the delivery of active agents without the constraint of radiation damage to normal tissue. The aim of this presentation is to summarise the potential of cold plasma to treat certain cancers and to outline the proposed instrumentation for in vitro treatment to verify the efficacy, before progressing to clinical trials.

Method The cell death for cell lines cultured in vitro and exposed to radiation and to direct plasma was compared (Figure 1, adapted from [1]). The concentration of RONS caused by radiation therapy and by plasma exposure was determined by simulation with input from the literature. The distribution of RONS created by the plasma exposure was calculated from Brownian diffusion. The information concerning equivalent doses was used to formulate a new design of a plasma system to be used in in vitro studies and to support potential use in clinical trials.

Results Our new plasma treatment system design is intended for use in a hospital based laboratory. It has two applications: the first is the exposure of cell lines for preclinical in vitro studies and the second is the exposure of biologically compatible solutions to enable pilot clinical trials.

Conclusion Cold plasma treatment offers new opportunities as a potential replacement or supplement for existing cancer therapies.

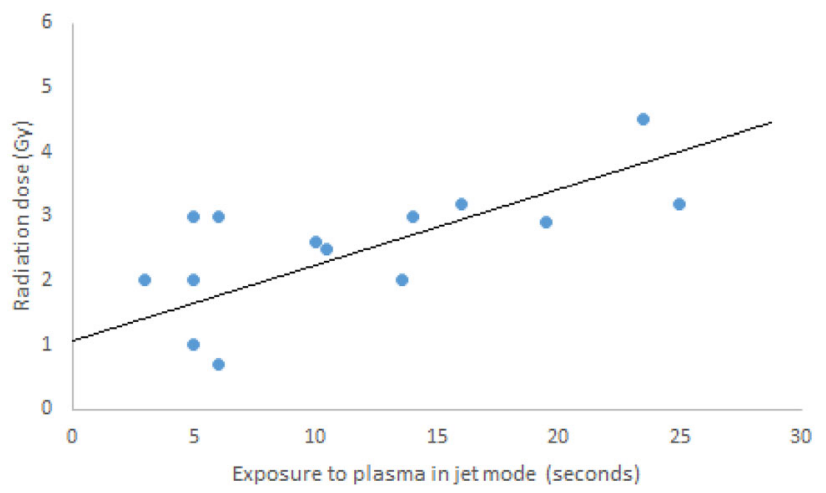

Figure 1 For a range of breast cancer cell lines the dose required to reduce the growth rate by $50 \%$ for exposure to radiation (Gy) and plasma in jet mode (seconds). Each point represents a single type of breast cancer (cell line). Adapted from [1]

Acknowledgements Australian Government Funding for the Chris O'Brien Lifehouse Sarcoma Research Centre. Sydney Breast Cancer Foundation.

\section{References}

1. Lafontaine, J., Boisvert, J.-S., Glory, A., Coulombe, S., and Wong, P. (2020). Synergy between non-thermal plasma with radiation therapy and olaparib in a panel of breast cancer cell lines. Cancers, 12(2):348. https://doi.org/10.3390/cancers 12020348

\section{P001 Shell properties of a new acoustofluidic delivery agent}

\author{
H A AL-Sadiq ${ }^{1}$, K R Tupally ${ }^{2}$, H S Parekh ${ }^{2}$, M Veidt ${ }^{1}$ \\ ${ }^{1}$ School of Mechanical and Mining Engineering, University \\ of Queensland. (h.alsadiq@uq.edu.au). ${ }^{2}$ School of Pharmacy, \\ University of Queensland. (k.tupally@uq.edu.au), \\ (h.parekh@uq.edu.au), (m.veidt@uq.edu.au)
}

Introduction Acoustofluidic manipulation of drug vehicles such as microbubbles and echogenic liposomes ELIP, has promising theranostic applications. A new acoustofluidic delivery agent that has an extended echogenic and number concentration stability $[1,2]$, has shown promising acoustofluidic enhanced intravitreal drug delivery potential in an ex vivo bovine eye [3]. The shell stiffness $S_{p}$ and shell friction $S_{f}$ coefficients of the agent are investigated as a prerequisite to studying ELIP translation due to sonication.

Method Frequency dependent attenuation measurement of an acoustic beam through a suspension of liposomes at room temperature and at $37{ }^{\circ} \mathrm{C}$ were performed under constant $11 \mathrm{kPa}$ pressure excitation to avoid influences of frequency dependent transducer performance and non-linear bubble deformations. The acquired data 


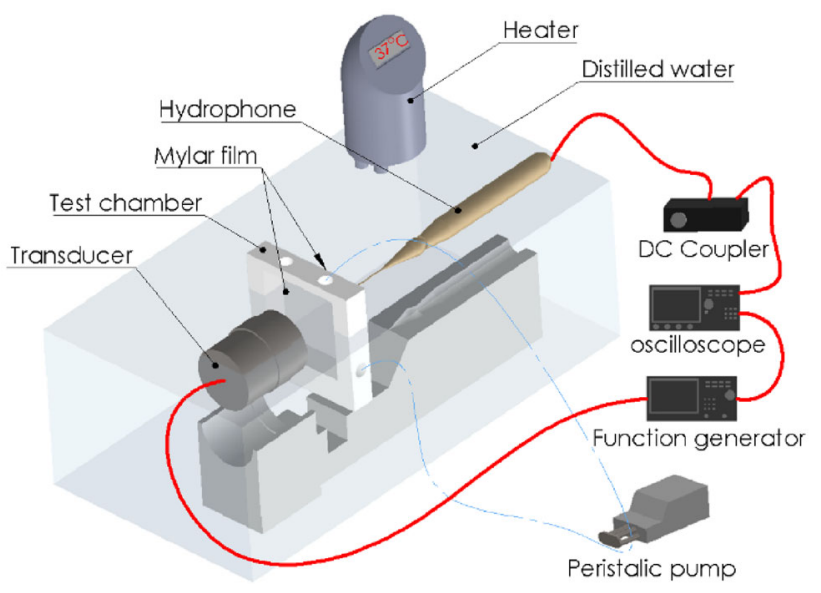

Figure 1 Schematic of the experimental setup

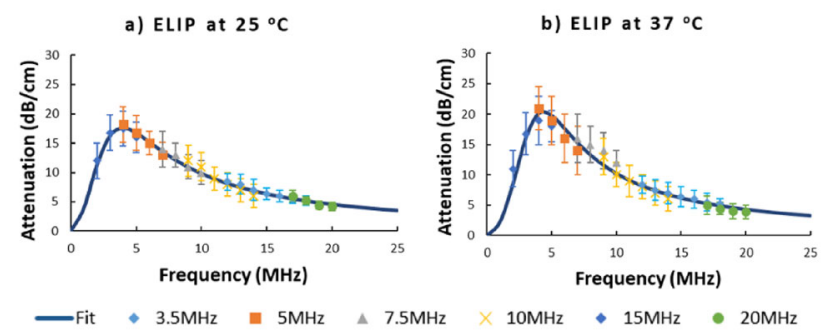

Figure 2 Attenuation experiment results and best fit for the ELIP at (a) room temperature and (b) physiological temperature

were analysed using an established theoretical model described in details in [4], to calculate the shell parameters.

The agents were formulated as described in [5], and their size distribution and concentration were measured using Tuneable Resistive Pulse Sensing as in [2]. The bubbles solution was then diluted by 1:20 and placed in a test chamber between an ultrasonic transducer and hydrophone, Figure 1. The transducer was excited using an arbitrary function generator at a frequency range of $2-20 \mathrm{MHz}$ and the pressure after passing the bubbles suspension was acquired. A peristaltic pump circulated the bubbles in the test chamber between tests to compensate for buoyancy effects.

Results The frequency dependent attenuation experimental results and the best fit are shown in Figure 2. The new bilayer acoustofluidic delivery agent shell parameters were calculated as $\left({ }^{\circ} \mathrm{C}\right)$ and $\left({ }^{\circ} \mathrm{C}\right)$.

Conclusion The new echogenic liposome shell parameters calculated in this study show very low stiffness and friction coefficients despite their extended stability, which makes them unique agents for acoustofluidic delivery applications.

\section{References}

1. Thakur SS, Ward MS, Popat A, Flemming NB, Parat M-O, Barnett NL, Parekh HS (2017) Stably engineered nanobubbles and ultrasound-An effective platform for enhanced macromolecular delivery to representative cells of the retina. PloS one 12 (5): $\mathrm{e} 0178305$

2. Alsadiq H, Tupally K, Kokil G, Vogel R, Parekh H, Veidt M (2019) Concentration stability of monolayer and bilayer lipid microbubbles-a precursor for new drug delivery applications.

3. Thakur SS, Chen Y-S, Houston ZH, Fletcher N, Barnett NL, Thurecht KJ, Rupenthal ID, Parekh HS (2019) Ultrasoundresponsive nanobubbles for enhanced intravitreal drug migration:
An ex vivo evaluation. European Journal of Pharmaceutics and Biopharmaceutics 136:102-107

4. Goertz DE, de Jong N, van der Steen AF (2007) Attenuation and size distribution measurements of Definity ${ }^{\mathrm{TM}}$ and manipulated Definity ${ }^{\mathrm{TM}}$ populations. Ultrasound in medicine \& biology 33 (9):1376-1388

5. Parekh H, Thakur S (2018) Method for Preparing a Lipid Bubble. Australia Patent WO/2018/053601.

\section{P002 Transformation of general ward to negative pressure ward in public hospital in the combat against COVID-19}

\section{Ir Y.W. $\mathrm{Ng}^{1}$, Ir Dr P.L. Yuen ${ }^{2}$, Ir W.T. Yip ${ }^{1}$}

${ }^{1}$ Electrical and Mechanical Services Department, The Government of the HKSAR. (aliceng@emsd.gov.hk [Presenting author]), (wtyip@emsd.gov.hk). 2The Hospital Authority, Hong Kong. (yuenpl@ha.org.hk)

Introduction During the outbreak of COVID-19 in 2020, there is a high demand of isolation rooms in hospitals for patients. Considering that it is technically not feasible for conducting major modification of ventilation system in hospitals within a short period of time, a fasttrack and cost-effective transformation approach was developed to convert general ward to negative pressure ward in public hospitals in Hong Kong to cope with the upsurge in suspected cases of COVID19.

Method A Mobile Modular High-Efficiency Particulate Air Filter Unit (MMHU) was developed to transform a general ward to a negative pressure ward quickly and with low-cost. The MMHU is a tailor-made mobile air purification unit with High-Efficiency Particulate Air (HEPA) filter and resembles a large air cleaner with exhaust fan and HEPA filter to extract indoor air from ward area through opening in window to outdoor, thereby creating a negative pressure inside the ward.

Results MMHUs were deployed to public hospitals and field tests including pressure difference measurement and smoke test were conducted. The results proved that a general ward transformed with MMHUs met the reference parameters advised by the Guidelines for Environmental Infection Control in Health-care facilities, and the use of MMHU created an inward flow from the corridor to the ward, thus preventing cross-infection inside the ward. The use of MMHU could minimize the major modification of ventilation system with traditional means of conversion and shorten the conversion time. It also helps to ease public concern on the air exhausted from wards.

Conclusion The use of MMHU is able to transform a general ward to a negative pressure ward within a short period of time and such transformation is considered effective in increasing the isolation facilities capacity in hospitals during the outbreak of infectious diseases.

\section{P003 Higher Degree Research degree pathways for the practising Biomedical engineer - a kaleidoscope of case studies}

\author{
R L G Kirsner ${ }^{1}$, P.A. Junor ${ }^{2}$ \\ ${ }^{1}$ (rkirsner@bigpond.com). ${ }^{2}$ La Trobe University. \\ (paul.junor@gmail.com)
}


The COVID pandemic has highlighted the fact that clinical engineering has evolved from being management of instrumentation maintenance to playing a significant role in the medical team and the health system.

Training beyond basic undergraduate studies is necessary to perform this role competently and effectively: among practising Clinical Engineers there has been an increasing awareness of the need to upskill to manage changes in technology.

Our experience supervising working biomedical engineers completing part-time higher degrees in research confirms our view that optimal biomedical engineering education programs need to be a full five years in duration.

Typical projects involve part-time higher degree by research students working on clinical projects hosted by hospitals/ research establishments, in which some of the candidates are also performing their normal work.

Informal associations with clinicians and requests for collaboration in projects are an important part of this process.

Currently in Australia there are more than ten biomedical engineering university degree courses [1].

To remain on par with the large number of graduates being produced from these courses, experienced Clinical Engineers practising in the workforce are seeking to upgrade their qualifications to postgraduate level.

This paper discusses a number of higher degree projects that illustrate these points.

Reference

1. https://www.engineersaustralia.org.au/About-Us/Accreditation

\section{P004 Engaging with a rapidly changing risk environment: how the Australian Clinical Dosimetry Service responded to CoVID-19}

\section{M Williams, J E Lye}

Australian Radiation Protection and Nuclear Safety Agency, Australia. (Ivan.williams@arpansa.gov.au [Presenting author]), (Jessica.Lye@arpansa.gov.au)

Introduction On the 17th March, with an unknown potential of a SARS-like virus beginning to impact Australia and a logarithmic increase in new cases nationally, the ACDS team was instructed to work from home until further notice. Five days later on Sunday 22, all audits were grounded until further notice. This included a Level Ib audit which was scheduled the following day on a newly installed linac. The ACDS, in collaboration with subscribers and regulators, has established new protocols enabling safe auditing to recommence. Method Operationally, facilities scheduled for on-site Level II and III audits were notified two weeks prior that they would be re-scheduled and that the conditions of audit would be modified to minimise the likelihood of infection. The Level I mail-out audit for Qtr 1 was being returned for read-out during this time. Returned audit kits were disinfected prior to being picked up and taken to the responsible staff member's home, where the readout equipment had been relocated. Strategically, the jurisdictional regulators and the Department of Health Division responsible for managing the Radiation Oncology Health Program Grants (ROHPG) were contacted and the CoVID-19 impact was discussed.

Regulators recognised the necessity of auditing to ensure patient safety and all agreed that they would support recommencing audits in a controlled ramp-up. DoH recognised that CoVID-19 would affect the audit schedule, and would monitor developments.
Results An entire new set of procedures for auditing staff was developed and for the first time, some audits were performed with remote support.

The first audit post grounding was on the 12 April in a jurisdiction with closed borders.

Audit teams of two ACDS staff were assembled to work together and no team performs more than one audit trip a week.

Conclusion The new normal is a constant change. The ACDS must respond quickly and appropriately to fulfil its important role in Australian radiation safety.

\section{P006 Modelling radiobiological response of paediatric cranial tumours following intensity modulated proton and photon radiation therapies - radiosensitivity and gender dependence}

\section{Mikaela Dell'Oro ${ }^{1,2}$, Michala Short ${ }^{1}$, Puthenparampil Wilson ${ }^{2,3}$, Eva Bezak ${ }^{1,4}$}

${ }^{1}$ Cancer Research Institute, University of South Australia, Adelaide, SA 5001, Australia. ${ }^{2}$ Department of Radiation Oncology, Royal Adelaide Hospital, Adelaide, SA 5000, Australia.

(mikaela.delloro@mymail.unisa.edu.au [Presenting author]), (michala.short@unisa.edu.au),

(puthenparampil.wilson@unisa.edu.au), (eva.bezak@unisa.edu.au).

${ }^{3}$ UniSA STEM, University of South Australia, Adelaide, SA 5001, Australia. ${ }^{4}$ Department of Physics, University of Adelaide, Adelaide, SA 5005, Australia

Introduction Medulloblastoma is the most diagnosed paediatric malignancy of the central nervous system. Despite the high number of paediatric patients treated with intensity-modulated photon (IMRT) and proton (IMPT) radiotherapy, risk factors for increased normal tissue complication probability (NTCP) are still not fully understood. Disparities in intrinsic radiosensitivity $(\alpha / \beta$ ratio) for paediatric patients exist, influencing NTCP outcomes. Additionally, literature suggests that TD50 can be $\sim 20 \%$ lower in female patients. Following this data, our aim was to model the impact of gender and intrinsic radiosensitivity on paediatric brain tumour IMRT and IMPT outcomes.

Method 216 comparative IMRT and IMPT plans were created for six paediatric patients, using Varian Eclipse software. Relative-seriality and Lyman-Kutcher-Burman models were used to calculate NTCP values for cranial irradiation of several anatomical structures.

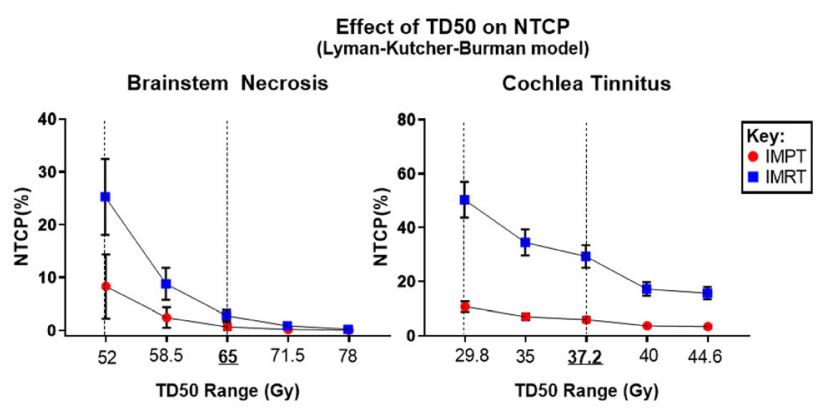

Graph 1 Effect of TD50 on NTCP of brainstem necrosis and cochlea tinnitus for medulloblastoma patients. TD50 for females is approximately $20 \%$ lower than males, this is represented dashed line compare to the second line (underlined TD50) which illustrates the mean Therefore, females will potentially have a higher NTCP for the given dose distribution 


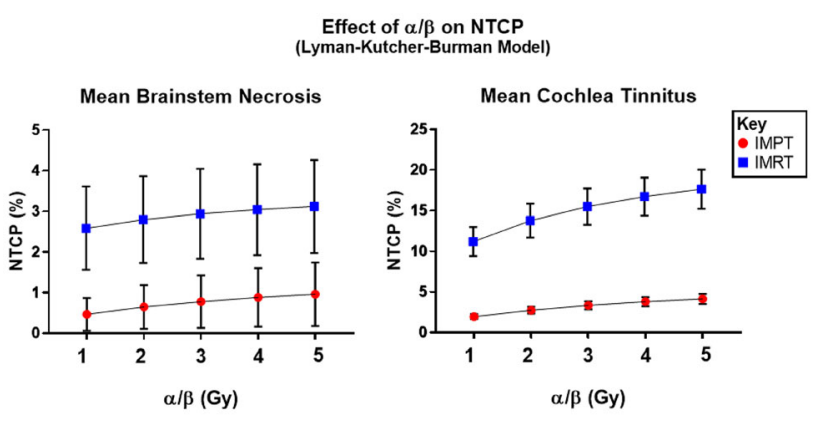

Graph 2 Effect of $\alpha / \beta$ on NTCP of brainstem necrosis and cochlea tinnitus for medulloblastoma patients

Evaluated complication endpoints included brainstem necrosis, blindness, and deafness. Sensitivity analyses were performed to gauge the impact of $\alpha / \beta$ values and TD50 on radiation response.

Results IMPT plans demonstrated smaller side-effect risks compared to IMRT across all NTCP models. For medulloblastoma, tinnitus and brainstem necrosis NTCP depended on modality and TD50 of irradiated normal tissue, therefore NTCP could be potentially underestimated in female paediatric patients (Graph 1). Similarly, IMPT demonstrated advantages for NTCP across all tested $\alpha / \beta$ values compared to IMRT, with lower $\alpha / \beta$ values estimating a reduced risk of adverse side effects (Graph 2). If the true $\alpha / \beta$ of these paediatric tissues are higher than predicted $(\alpha / \beta \sim 3)$, planning could severely increase risk of cochlea tinnitus for IMRT. In general, NTCP for IMPT was not as influenced when changing parameters in the model compared to IMRT.

Conclusion This study aimed to estimate the impact of gender on NTCP for paediatric cranial critical structures. Modified NTCP calculations can be used for ranking of treatment plans to better stratify patients who would benefit most from IMPT.

Acknowledgements St. Jude Children's Research Hospital.

\section{References}

De Courcy L, Bezak E, Marcu LG. Gender-dependent radiotherapy (2020) The next step in personalised medicine? Crit Rev Oncol Hematol 147:102881. https://doi.org/10.1016/j.critrevonc.2020. 102881

Snyder M, Burmeister J, Joiner M, et al. Potential Gender Differences in a Normal Tissue Complication Probability Model for Heart Toxicity During Radiation Therapy for Esophageal Cancer (2012) Int. J. Radiat. Oncol. Biol. Phys 84(3): S757-S758. https://doi.org/ 10.1016/j.ijrobp.2012.07.2026

\section{P007 In vitro $\mathrm{pH}$ measurement by proton magnetic resonance spectroscopy and glucose chemical exchange saturation transfer}

\author{
Neda Gholizadeh ${ }^{1}$, Peter B. Greer ${ }^{1,2}$, Saadallah Ramadan ${ }^{1,3}$, \\ Jonathan Goodwin ${ }^{1,2}$ \\ ${ }^{1}$ Faculty of Science, The University of Newcastle, Australia. \\ (neda.gholizadeh@uon.edu.au [Presenting author]), \\ (saadallah.ramadan@newcastle.edu.au). ${ }^{2}$ Calvary Mater Newcastle, \\ Radiation Oncology, Australia. (peter.greer@newcastle.edu.au), \\ (Jonathan.Goodwin@calvarymater.org.au). ${ }^{3}$ Hunter Medical \\ Research Institute (HMRI) Imaging Centre, Australia
}

Introduction The microenvironment of many solid tumours can be acidic, reaching an average extracellular $\mathrm{pH}$ value of approximately 6-7.0 [1]. Tumor $\mathrm{pH}$ is physiologically important since it influences a

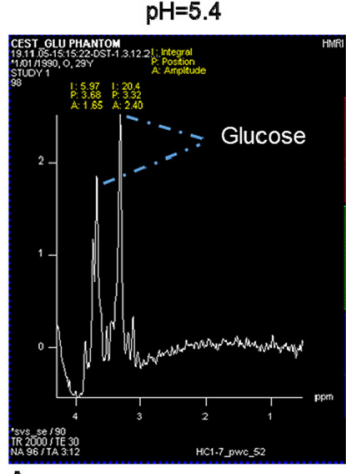

A

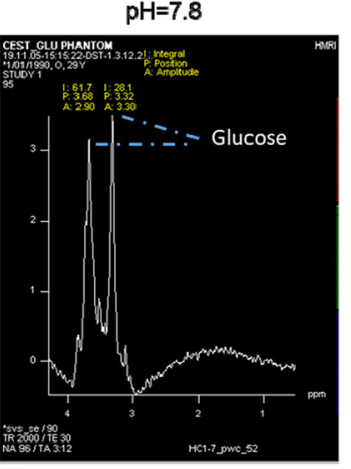

B
Figure 1 Examples of protons magnetic resonance spectroscopy $(1 \mathrm{H}$ MRS) for two different phantoms with A) $\mathrm{pH}$ of 5.4 and $\mathrm{B}$ ) $\mathrm{pH}$ of 7.8, respectively

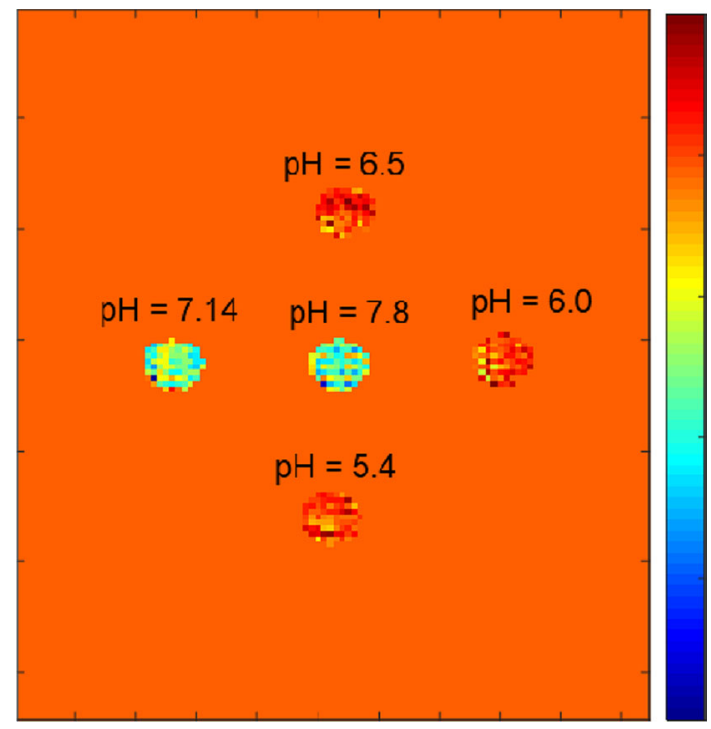

Figure 2 glucoCEST contrast obtained from five phantoms containing $20 \mathrm{mM}$ of glucose at different $\mathrm{pH}$ values 5.4, 6.0, 6.5, 7.1 and 7.8

number of processes relevant to metastasis and treatment management [2]. Proton magnetic resonance spectroscopy $\left({ }^{1} \mathrm{H}\right.$ MRS) and chemical exchange saturation transfer (CEST) are two sensitive techniques that provide metabolic information. The aim of this study was to evaluate the feasibility of $\mathrm{pH}$ measurement in vitro using ${ }^{1} \mathrm{H}$ MRS and glucoCEST.

Method ${ }^{1} \mathrm{H}$ MRS and glucoCEST were performed on a 3T MR scanner. Five phantoms containing $20 \mathrm{mM}$ of glucose were prepared at different $\mathrm{pH}$ values 5.4, 6.0, 6.5, 7.1 and 7.8. Model solutions were buffered using phosphate-buffered saline (PBS). Sodium-azide was used to suppress the growth of gram-negative organisms. All model solutions were pipetted from highly concentrated stock solutions to ensure a precise adjustment of the concentrations, and $\mathrm{pH}$ values were adjusted to the final values using hydrochloric-acid $(\mathrm{HCl})$ at $92 \%$ of the final volume, to take into account the added amounts of HCl. Final $\mathrm{pH}$ values were checked by means of a calibrated $\mathrm{pH}$ electrode.

Results There was no significant difference in resonances of glucose signals derived from ${ }^{1} \mathrm{H}$ MRSI for different $\mathrm{pH}(p>0.05)$ (Figure 1). For CEST imaging, the field inhomogeneity correction was performed by interpolating and shifting the $\mathrm{Z}$-spectra based on the obtained $\mathrm{B}_{0}$ 
field map. The optimal glucoCEST contrast obtained with RF power of $3 \mu \mathrm{T}$ in the current study (Figure 2). Glucose peaks in CEST $\mathrm{Z}$-spectra of the phantoms were observed between $1.1 \mathrm{ppm}$ and 2.9 ppm. The CEST contrast showed a significant negative correlation with $\mathrm{pH}(\mathrm{r}=-0.87$ and $\mathrm{p}<0.01)$.

Conclusion These results demonstrated the potential role of glucoCEST for microenvironment $\mathrm{pH}$ mapping in vivo. Acquisition parameters optimization is necessary to obtain the maximum glucoCEST imaging contrast.

\section{References}

1. Zhang, Xiaomeng, Yuxiang Lin, and Robert J. Gillies (2010). Tumor $\mathrm{pH}$ and its measurement. Journal of Nuclear Medicine, vol. 51, no. 8, pp. 1167-1170

2. Tannock, Ian F., and Daniela Rotin (1989). Acid pH in tumors and its potential for therapeutic exploitation. Cancer research, vol. 49 , no.16, pp. 4373-4384

\section{P008 Ring Applicator Dwell Position Uncertainty: reproducible and accurate source position determination for cervix brachytherapy.}

\section{Marsh}

The Townsville Cancer Center, Townsville University Hospital, QLD. (Linda.Marsh@health.qld.gov.au [Presenting author])

Introduction The ring applicator is used for high dose rate afterloaded brachytherapy of the uterine cervix. The Iridium high dose rate source is welded to a cable that is unwound by precise amounts by a step motor to control the placement of the source in the applicator. Being circular with a small radius and narrow lumen, the ring modifies source displacement. To predict the location of source for accurate dose calculation a factory source path is used. The aim of this study was to characterise the reproducibility of the placement of the source for a ring applicator and determine the accuracy of the factory source path.

Method The ring applicator was fixed to EBT3 film in a rigid assembly and radiographed using the superficial unit to deliver exposure adequate for visualizing the internal structure. The rigid assembly was transferred to the brachytherapy suite and autoradiographed with the Iridium source at nominal dwell positions spanning the circumference of the applicator. Image analysis was conducted using Excel spreadsheets and commercial film analysis software. Several repetitions of the process were performed for assessment of reproducibility of the source location.

Results The 17 dwell positions studied were located with a mean uncertainty of $1 \mathrm{~mm}$ and were on average $0.6 \mathrm{~mm}$ distant from the positions specified by the factory source path. Ring sections were identified, the connector where the ring joins the handle, a bunching section where the ring modifies the movement of the source causing a bunching of dwells and the clinical section. Uncertainty of the source position was up to $3.1 \mathrm{~mm}$ in the connector section and $1.1 \mathrm{~mm}$ in the bunching section.

Conclusion Dwell positions in the clinical ring segments were verified to be accurate and reproducible, whereas dwell positions in the bunching and connector sections were less reproducible.

\section{References/Acknowledgements}

1. Awunor B, Dixon B, and Walker C, Direct Reconstruction and associated uncertainties of 192Ir source dwell positions in ring applicators using gafchromic film in the treatment of HDR brachytherapy cervix patients, Phys. Med. Biol., pp. 3207-3225, 2013.
2. Awunor B, Berger D, and Kirisits C, A multicenter study to quantify systematic variations and associated uncertainties in source positioning with commonly used HDR afterloaders and ring applicators for the treatment of cervical carcinomas, Medical Physics, vol. 42, no. 8, pp. 4472-4483, 2015.

3. Fonseca G, Van den Bosck M, Voncken R, Podesta M, Verhaegen F, A novel system for commissioning brachytherapy applicators: example of a ring applicator, Phys. Med. Biol., vol. 62, pp. 8360-8375, 2017.

4. Humer I, Kirisits C, Berger D, Trnkova P, Potter R, Nesvacil N, Improved source path localisation in ring applicators and the clinical impact for gynecological brachytherapy, Journal of Contemporary Brachytherapy, vol. 7, no. 2, pp. 239-243, 2015.

5. BIPM, Evaluation of measurement data - Guide to the expression of uncertainty in measurement, JCGM, 2008.

6. Dempsey C, Smith R, Nyathi T et al, ACPSEM brachytherapy working group recommendations for quality assurance in brachytherapy., vol. 36, no. 4, 2013.

\section{P009 Depth dose measurement through a high-density block in a water tank demonstrating agreement with Dose to Water as calculated by a voxel based TPS.}

\section{N Bennie}

North Coast Cancer Institute, Lismore, NSW, Australia. (Nick.Bennie@ncahs.health.nsw.gov.au [Presenting author])

Introduction It has been noted that that when using the "Dose to water" option of a voxel based TPS to calculate a depth dose graph through a HD block there is a rapid rise in dose at the boundary of normal density water to a high-density block of bone like material. This rapid rise has been considered not realistic. This paper presents

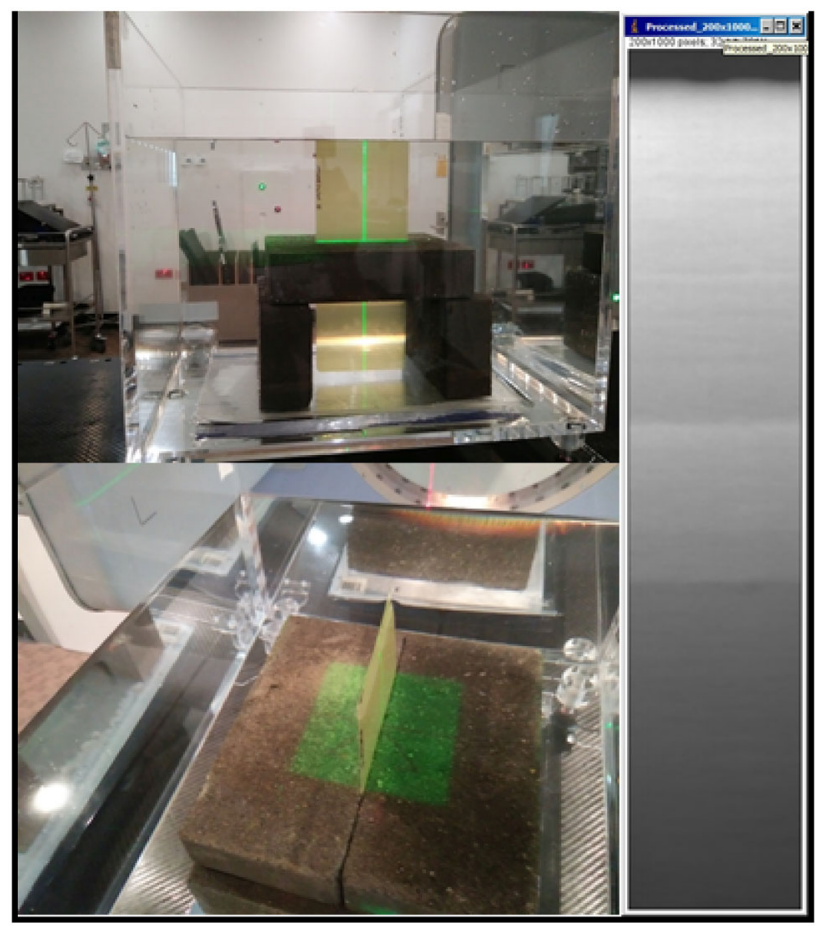

Figure 1 Experimental setup 


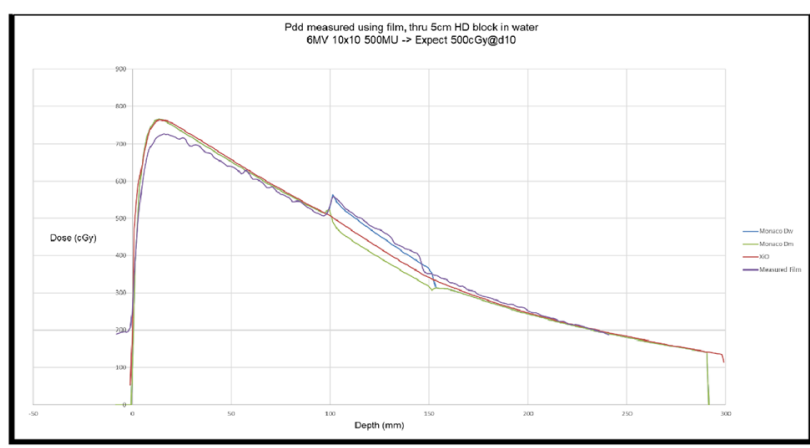

Figure 2 Depth dose measured using film, thru $5 \mathrm{~cm}$ HD block in water

an experimental setup and measurement data which are in agreement with "Dose to Water" as calculated by the Elekta Monaco TPS. That is, the rapid change in dose at material boundaries is, in fact, supported by experimental evidence.

Method Dense bone like material was approximated by cement blocks. Water like soft tissue was approximated by liquid water. GafChromic EBT3 film was cut into a strip. This extended from the water surface to a depth of $25 \mathrm{~cm}$. At depth $10 \mathrm{~cm}$ a HD block was placed either side. A beam entering from surface traversed $10 \mathrm{~cm}$ water, $5 \mathrm{~cm}$ HD block, $10 \mathrm{~cm}$ water (Figs. 70, 71).

Results Note the change seen at boundaries is rapid but continuous, changing on approximately same scale as range of HE Electrons set in motion from MV radiation. The measurement is in good agreement with the "Dose to water" calculation.

This can be contrasted with the "Dose to Medium" calculation which exhibits a step change at the material boundary.

Conclusion The standard interpretation of radiation interacting with a dosimeter is supported. That is, the material of the dosimeter responds to the radiation fluence. The radiation fluence is greater in the HD bone like material than in the water, hence greater response. The active medium of GafChromic film is designed to be equivalent to water, therefore the response of the film being in agreement with the "Dose to water" calculation of the TPS is as expected.

\section{References}

1. Khan, F. M. (1994). The Physics of Radiation Therapy. $2^{\text {nd }}$ Edition. Lippincott Williams \& Wilkins. (ISBN 0-683-04502-4)

2. ICRU Report 83. State of the art on dose prescription, reporting and recording in intensity-modulated radiation therapy. ICRU 2011 (Bethesda, MD)

3. ICRU Report 85. Fundamental quantities and units for ionizing radiation. ICRU 2011 (Bethesda, MD)

4. Elekta (Elekta AB, Stockholm, Sweden.) documents including: Monaco Dose Calculation Technical Reference and letter titled "SUMMARY OF DOSE TO WATER CALCULATIONS IN MONACO (V3.1 and later)" dist. Feb 2013.

5. Ma, C. M., \& Li, J. (2011). Dose specification for radiation therapy: dose to water or dose to medium?. Physics in Medicine \& Biology, 56(10), 3073.

6. Bennie, N., \& Metcalfe, P. (2016). Practical IMRT QA dosimetry using Gafchromic film: a quick start guide. Australasian physical $\&$ engineering sciences in medicine, 39(2), 533-545.
P010 It is not possible to create a homogeneous "dose to medium" distribution in a heterogeneous patient. Is this a problem?

\section{N Bennie}

North Coast Cancer Institute, Lismore, NSW, Australia. (Nick.Bennie@ncahs.health.nsw.gov.au [Presenting author]

Introduction For MV radiation, it is simple to demonstrate that for a "Dose to medium" calculation of a TPS using a small voxel size, less than range of high energy electrons, that if the "medium" of two adjacent voxels are different, then the value of dose derived for these two voxels will be different by the MSPR of the two materials that constitute the "medium" of the respective voxels. This in itself is a trivial conclusion, however applying this principle to real patient plans where there are one or more regions of significantly different material within a PTV, then it can be concluded that for certain patients, it will not be possible to delivery a homogeneous "Dose to Medium" distribution.

For different TPS and different optimisation criteria, different consequences of this effect can be observed. However, for all EBRT, optimization is the adjustment of the primary beam through the collimator (MLC). All TPS optimisations using "Dose to Medium" calculation will exhibit unstable behavior when they encounter heterogeneous material distributions, in particular for clinical patients, bone to soft tissue, within the PTV (Fig. 72).

For the Elekta Monaco TPS, this may result in the optimization not being able to achieve an acceptable result (fail), or exhibited as an overall increase in total radiation exposure to the patient. That is, an increase in radiation to uninvolved tissue when "Dose to Medium" is used compared to an equivalent plan optimised using "Dose to water".

Method Patient plans were developed on the Elekta Monaco TPS using both "Dose to Medium" and "Dose to Water" options.

\section{Results}

Conclusion It is concluded, that it is a problem to use "Dose to Medium" calculation for optimization. It can be unstable and overall, always results in a greater total radiation exposure to the patient.

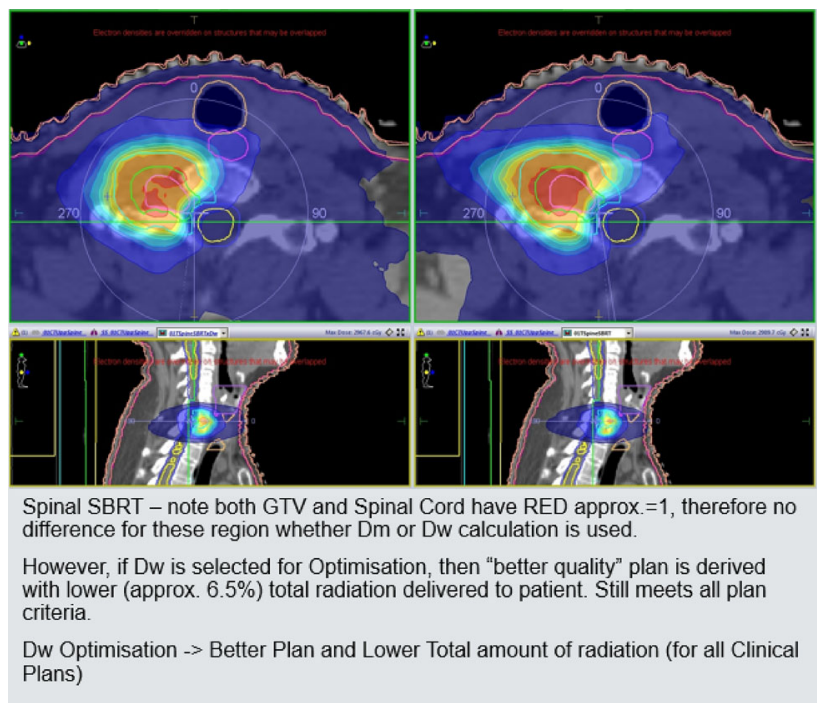

Figure 1 Results of equivalent "Dose to Water" vs "Dose to Medium" optimisations 


\section{References}

1. Khan, F. M. (1994). The Physics of Radiation Therapy. $2^{\text {nd }}$ Edition. Lippincott Williams \& Wilkins. (ISBN 0-683-04502-4)

2. ICRU Report 83. State of the art on dose prescription, reporting and recording in intensity-modulated radiation therapy. ICRU 2011 (Bethesda, MD)

3. ICRU Report 85. Fundamental quantities and units for ionizing radiation. ICRU 2011 (Bethesda, MD)

4. Elekta (Elekta AB, Stockholm, Sweden.) documents including: Monaco Dose Calculation Technical Reference and letter titled "SUMMARY OF DOSE TO WATER CALCULATIONS IN MONACO (V3.1 and later)" dist. Feb 2013.

5. Ma, C. M., \& Li, J. (2011). Dose specification for radiation therapy: dose to water or dose to medium?. Physics in Medicine \& Biology, 56(10), 3073.

\section{P012 Evaluation of Ion recombination factors for various cylindrical chambers using multiple methods}

\section{J Daniel, J Zifodya, Y Yousif, J Collins, W-L Hsieh}

North West Cancer Centre, Tamworth, Australia. (John.Daniel@health.nsw.gov.au [Presenting author]), (Jackson.Zifodya@health.nsw.gov.au),

(Yousif.Yousif@health.nsw.gov.au), (John.Collins1@health.nsw.gov.au), (Wen-

Long.Hsieh@health.nsw.gov.au)

Introduction Incomplete charge collection due to the ion recombination, $\mathrm{P}_{\text {ion }}$, in ionisation chamber requires a correction factor for accurate dose measurements. Generally, three main factors affecting the $\mathrm{P}_{\mathrm{ion}}$ in a pulsed Linac's beam which are: applied polarising chamber voltage, plate separation and the dose per pulse (DPP). The purpose of this study was to investigate the $\mathrm{P}_{\text {ion }}$ correction factors for three different ionisation chamber models under conventional flattened beams as well as in FFF beams using three methods.

Methods $\mathrm{P}_{\text {ion }}$ factors were measured using Jaffe' plots, two-voltage method and a semi-empirical method derived from the Boag theory ${ }^{1,2}$ which utilises the dose per pulse from the linac for three PTW type chambers: Farmer TW30013, Semi-flex TW31010 and Pinpoint TW31016. Measurements were performed using a 10x10 $\mathrm{cm}^{2}$ field size at $100 \mathrm{SSD}$ and at $10 \mathrm{~cm}$ depth on the CAX for $6 \mathrm{MV}, 18 \mathrm{MV}$, and 6 FFF beams produced in a Varian Clinac IX. The measured $\mathrm{P}_{\text {ion }}$ factors were compared and validated against the published guidelines $^{2}$.

Results $\mathrm{P}_{\text {ion }}$ factors measured for the three ionisation chambers were found to be generally agreed with the published data ${ }^{1,2}$. The twovoltage and a semi-empirical methods indicated similar results. However, the Jaffe' plot showed variations of maximum value of 0.13 $\%$ from the other two-methods. It was also evident that $\mathrm{P}_{\text {ion }}$ factors were slightly varied for flattened beam and FFF beam among three chambers and this is most likely due to the effect of dose per pulse for different beams and detector size variations.

Conclusion It is necessary for all clinical departments to characterize their ionisation chambers before being utilized for clinical dosimetry. The study demonstrated that the two-voltage method can be considered as a suitable method for determining $\mathrm{P}_{\text {ion }}$ factors for FFF beams. References

1. Burns, D., \& McEwen, M. (1998). Ion recombination corrections for the NACP parallel-plate chamber in a pulsed electron beam. Physics in Medicine \& Biology, 43(8), 2033.
2. McEwen, M., DeWerd, L., Ibbott, G., Followill, D., Rogers, D. W., Seltzer, S., \& Seuntjens, J. (2014). Addendum to the AAPM's TG-51 protocol for clinical reference dosimetry of high-energy photon beams. Medical Physics, 41(4).

\section{P013 Feasibility of electron paramagnetic resonance with alanine dosimetry for use in clinical applications}

\author{
M B Hirning ${ }^{1}$, C Noble ${ }^{2}$, P Ramachandran ${ }^{2}$ \\ ${ }^{1}$ Radiation Oncology - Princess Alexandra Hospital Raymond \\ Terrace (ROPART), South Brisbane, Queensland, Australia. \\ (michael.hirning@health.qld.gov.au [Presenting author]). ${ }^{2}$ Princess \\ Alexandra Hospital, Brisbane, Australia. \\ (chris.noble@health.qld.gov.au), \\ (prabhakar.ramachandran@health.qld.gov.au)
}

Introduction In some materials the radiation dose may be determined by measuring the relative concentration of radiation generated free radicals using a technique known as electron paramagnetic resonance (EPR) spectroscopy. For radiation oncology, EPR alanine-dosimetry has clinical applications for small field dosimetry, in-vivo and in-vitro dosimetry. This study investigated the feasibility of implementing alanine dosimetry at the Princess Alexandria Hospital (PAH) using EPR spectroscopy. Currently the PAH has the only EPR spectrometer in Australia being assessed for clinical applications.

Method Standard Magnettech L- $\alpha$-alanine calibration pellets irradiated with $2 \mathrm{MeV}$ electrons from 1.022 Gy to $102.1 \mathrm{~Gy}$ were measured with a MiniScope MS 5000 EPR Spectrometer by Magnettech. EPR signal intensity was calibrated against known doses and the calibration curve was used to estimate the uncertainty of the EPR signal for dosimetry. A series of $\mathrm{D}-\alpha$-alanine dosimeters were irradiated in intervals of 7 Gy up to 84 Gy using $6 \mathrm{MV}$ photons. Four separate EPR signals were acquired for the capsules/tablets using two orientations (up/down) and two rotations $\left(0 / 180^{\circ}\right)$. Alanine EPR spectra were analysed using the double integral, peak-to-peak height and gradient of the main absorption peak to compare each technique for accuracy as an estimator of dose.

Results The initial dose uncertainty for the Standard L-Alanine pellets and D-Alanine capsules was found to be $3.9 \mathrm{~Gy}$ and $4.5 \mathrm{~Gy}$, respectively using the double integral analysis. Using progressive signal accumulation for doses less than $10 \mathrm{~Gy}$ these uncertainties for the standard pellets were reduced to 3.6 Gy using the double integral, 2.6 Gy using peak-to-peak and $2.5 \mathrm{~Gy}$ using the signal gradient method.

Conclusion The use of alanine dosimetry could be commissioned for clinical use provided the dose uncertainty is able to be minimised through either the use of a reference sample to correct for fluctuations in the spectrometer g-value. Alternatively, multiple reference dosimeters could be measured to correct the calibration curve for the g-value at the time of measurement. The dose should be measured using peak-to-peak values using progressively higher signal accumulation time for lower expected doses. The positioning of the dosimeter in the spectrometer sample holder produces a large uncertainty which can be minimised by fixing the dosimeter position within the spectrometer.

\section{References/Acknowledgements}

The author would like the thank Kaz Hosokawa, John Barry and Dr Sarah Walden from the Queensland university of Technology for their assistance in supplying some of the materials for this project. 


\section{P014 Combined electron paramagnetic resonance (EPR) and optically stimulated luminescence (OSL) investigation of the defect states in $\mathrm{BeO}$ dosimeter chips}

\section{Christopher Noble, Prabhakar Ramachandran}

Radiation Oncology, Princess Alexandra Hospital, Ipswich Rd, Brisbane, Australia. (Christopher.Noble@health.qld.gov.au [Presenting author]), (Prabhakar.Ramachandran@health.qld.gov.au)

Introduction The Princess Alexandra Hospital recently purchased a Lexsygsmart OSLD/TLD reader to provide in-vivo dosimetry within the department. The Lexsygsmart reader was supplied with $\mathrm{BeO}$ dosimeter chips and although they have good tissue equivalence and excellent energy independence they are not as well characterized as the more common $\mathrm{Al}_{2} \mathrm{O}_{3}$ dosimeter chips. The current bleach cycle appears to result in a progressive reduction in the sensitivity of the dosimeters. A thorough understanding of the defect states involved in the OSL signal is vital for developing bleaching/annealing protocols that would provide sufficient accuracy for routine in-vivo dosimetry. Method A BeO chip was first irradiated with 50 Gy using a $6 \mathrm{MV}$ $10 \times 10$ photon beam. The EPR spectrum was measured using a Magnettech MS5000 spectrometer. The peak-to-peak amplitude of the $\mathrm{Li}$ +-hole EPR center was monitored together with the OSL signal while bleaching with blue light in the OSL reader. Initially the chip was bleached with $30 \mathrm{~mW} / \mathrm{cm}^{2}$ blue light for 30s. At the end of the bleaching periods the OSL signal was measured and the chip was then placed in the EPR spectrometer for measurement. This was repeated 5 times. The chip was then bleached with the standard protocol with $100 \mathrm{~mW} / \mathrm{cm}^{2}$ blue light for 300s. Finally, the chip was heated to 260 ${ }^{\circ} \mathrm{C}$ for $300 \mathrm{~s}$

Results The EPR signal decreases in intensity progressively as the chip is bleached (Figure 1a). The final spectrum after the full bleach cycle shows that the EPR centre has not been fully bleached at this point. This can be seen more clearly when the peak-to-peak intensity is plotted together with the OSL signal as a function of the number of bleach cycles (Figure1b). Both the EPR and the OSL signals decay but at different rates. Annealing at $260{ }^{\circ} \mathrm{C}$ for 300 s appears to have effectively annealed out the EPR defect.

Conclusion These results show that the $\mathrm{BeO}$ dosimeters are not returned to their initial pre-irradiation state by the current bleach cycle. Approximately $30 \%$ of the EPR active defects produced by the irradiation remain after bleaching and this is related to the reduced sensitivity of the dosimeters after an irradiation/bleach cycle.

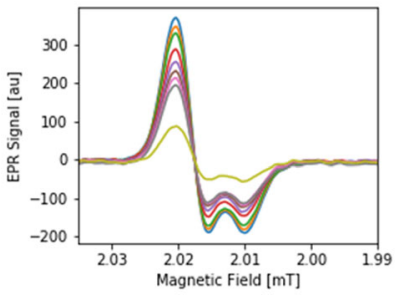

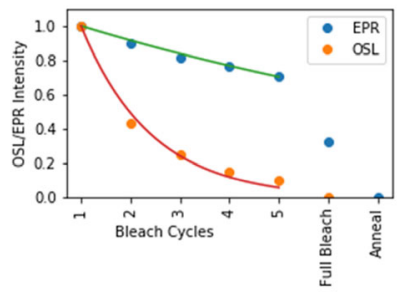

(b)
Figure 1 Combined EPR/OSL bleaching experiment a) The peak-topeak amplitude of the EPR spectrum decreases during optical bleaching but is not completely removed $b$ ) The OSL signal decreases much more rapidly than the EPR signal and is completely removed by the full bleach cycle
P015 Implementation of advanced radiotherapy technology to improve clinical outcomes in regional set up

D Banjade ${ }^{1}$, K Thuraisingam ${ }^{1}$, A Mishra ${ }^{1}$, S J (Eugene) Tan ${ }^{1}$, B Newham $^{1}$, A Renshaw ${ }^{1}$, Stephen How ${ }^{1}$, R Hammond ${ }^{1}$, G Stevens', G Warr ${ }^{2}$

${ }^{1}$ Department of Radiation Oncology, Central West Cancer Care Centre, Orange Hospital, Orange NSW 2800.

(Dilli.banjade@health.nsw.gov.au [Presenting author]). ${ }^{2}$ Department of Radiation Oncology, Mid North Coast LHD, Coffs Harbour NSW 2450

Introduction In Australia, radiotherapy (alone or with chemotherapy) is used for treating $48.3 \%$ of notifiable cancer patients and availability of advanced radiotherapy would have better clinical outcome. Cancer patients in remote areas experience poorer outcomes than their metropolitan counterparts with distance being an appreciable barrier to treatment access mainly to advanced techniques.

Problem At Central West Cancer Care Centre (CWCCC) the utilization rate of Intensity-Modulated Radiotherapy (IMRT) and Volumetric Modulated Arc Therapy (VMAT) was significantly lower compared to other NSW public health services. Stereotactic Ablative Body Radiotherapy (SABR) treatment was not available at CWCCC. Design Increase the IMRT/VMAT utilization rate and make SABR treatment available through quality improvement projects with multidisciplinary collaboration. A driver diagram (Figure 1) was developed to identify the challenges, drivers, change ideas and the aim of the project using recommended quality improvement tools from the clinical leadership program of clinical excellent commission of New South Wales.

Key measures for improvement Increase IMRT/VMAT utilisation rate; SABR available to CWCCC patients.

Strategy for change A multi-discipline team consists of Radiation Oncologists, Medical Physics Specialists and Radiation Therapists was formed and a project plan was developed to implement each treatment technique.

What this study adds?

1. State of the art treatment can be implemented in rural settings with a proactive and collaborative team

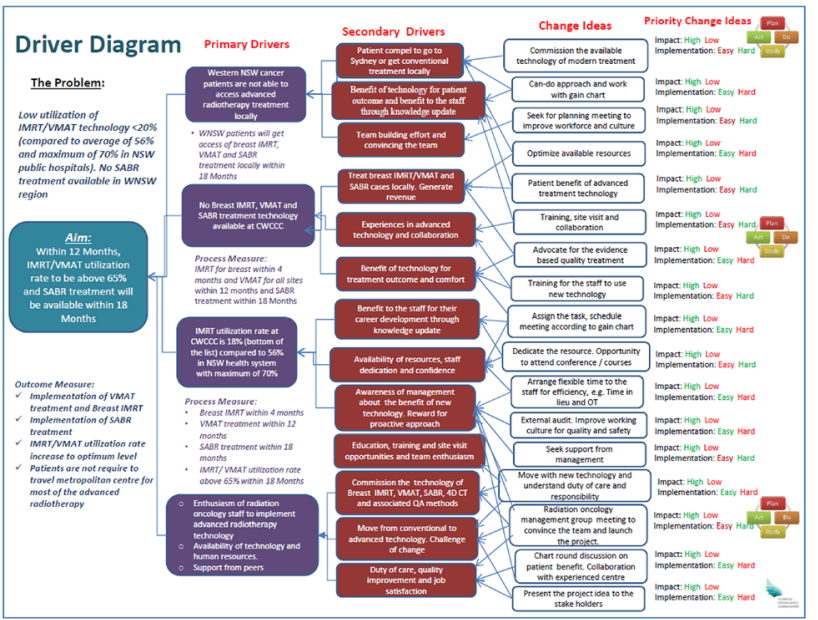

Figure 1 Technology implementation project Driver Diagram with detail including change ideas 


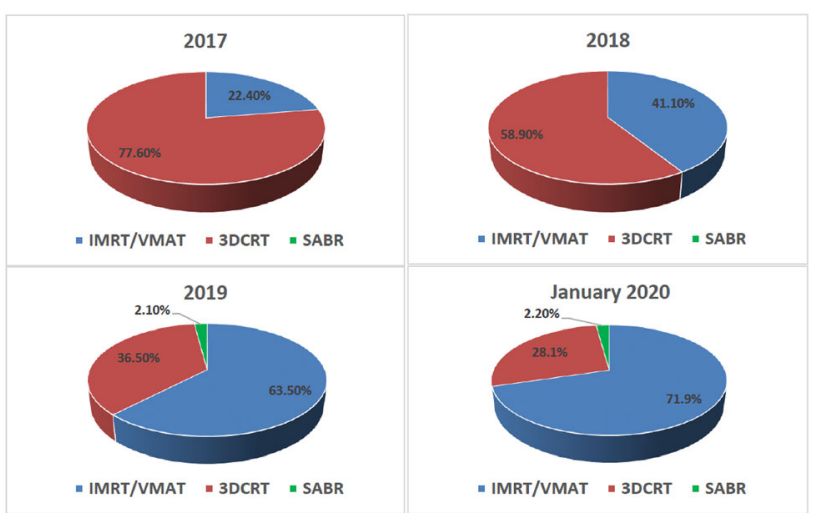

Figure 2 Breakdown of patient treatment modality at CWCCC

- Additional staff and/or extensive training are not always required to implement new treatments

- End-to-end testing to ensure safety and treatment quality is a critical part of practitioner confidence in new treatments.

Results After implementing Breast IMRT, VMAT and SABR treatment, the average IMRT/VMAT utilization rate at increases from $22 \%$ to $63 \%$ as shown in Figure 2. SABR treatment is also available at CWCCC from 2019. The radiation oncology team is working to further maximise the utilization rate in coming years with the promising data of IMRT/VMAT utilization rate of above $70 \%$ as shown in Figure in January 2020.

Conclusion Strong commitment from a trained team and a collaborative approach is important for the implementation of advanced technology in regional centres.

\section{Acknowledgements}

1. Professor Tomas Kron from the Peter McCallum Cancer centre and Professor Annette Haworth from the University of Sydney for their support to implement SABR program.

2. Organizer and Teachers from Executive Clinical Leadership Program- Cohort 23 of Clinical Excellence Commission of NSW government.

Note: The results are accepted for publication in Australian Journal of Rural Health (AJRH) as a quality improvement paper

\section{References}

1. Barton M, Jacob S and Shafiq J et al (2013). Review of optimal radiotherapy utilization rates. Ingham Institute for applied medical research. https://inghaminstitute.org.au/wp-content/uploads/2017/ 05/RTU-Review-Final-v3-02042013.compressed.pdf

2. Waran, E (2015). Radiotherapy: The Tyranny of distance. Aust. J. Rural Health: Volume (25): 64-65

3. RANZCR (2018).Techniques and Technologies in Radiation Oncology 2018, Version 1.0

4. Currow D, Thomson W and Bailey K (2018). Radiotherapy Treatment Services to NSW Residents: 2017 Annual Report; Cancer Institute NSW

5. CEC-CLP: http://www.cec.health.nsw.gov.au/quality-improvement/ improvement-academy/quality-improvement-tools

\section{P016 Characteristics of a single CT-ED Curve for all Varian Halcyon linear accelerators}

\author{
T Jarema, B Archibald-Heeren, M Byrne, Y Hu, S Towns, T \\ Aland
}

Icon Cancer Care Centres, Australia. (Talia.Jarema@icon.team [Presenting author]), (Ben.Archibald-Heeren@icon.team), (Mikel.Byrne@icon.team), (Yunfei.Hu@icon.team), (Sam.Towns@icon.team), (Trent.Aland@icon.team)

Introduction The Varian Halcyon iterative cone beam CT (iCBCT) has previously been shown to be a suitable substitute for a diagnostic CT scanner for the treatment planning of Pelvis patients [1]. As Halcyon linacs have preset iCBCT modes that cannot be changed by the user, all $\mathrm{kV}$ settings are identical. Our institution has 4 Halcyon linacs, all of which have been commissioned for treatment planning scans. The aim of this study was to compare the data from each linac to determine the feasibility of using a single CT-ED curve for all Halcyons, reducing commissioning times, standardising QA processes, and reducing the risk of incidents involving incorrect CT-ED curves being assigned.

Method The CT-ED data of the Pelvis mode from each Halcyon linac was averaged to create a single CT-ED curve. The results from each linac were then compared to the average to determine how large the uncertainty would be in both CT-ED curves. The CT-ED curve for other predefined iCBCT modes was also collected and the differences compared to the curve determined.

Results The comparison of CT-ED curves for the preset Pelvis iCBCT modality across 4 Halcyons is shown in Figure 1. The max-

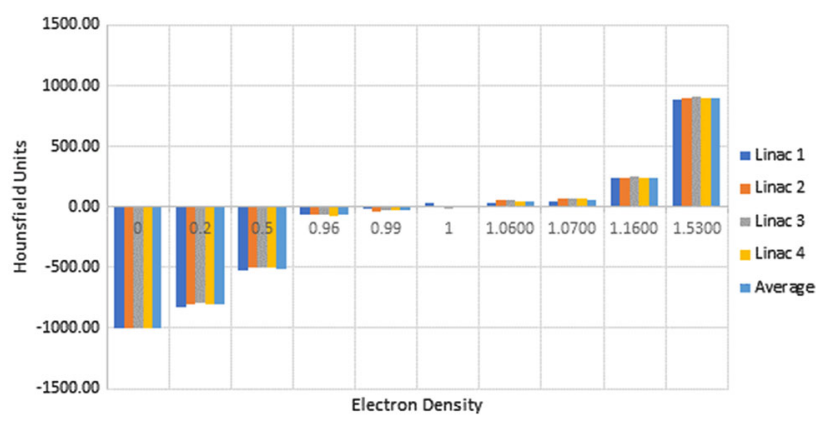

Figure 1 CT-ED curve comparison

imum difference between any datapoint on the CT-ED curve of any one linac was no more than $20 \mathrm{HU}$ from the average, with the largest differences observed in lower density materials.

Conclusion This study has shown that there is potential for a "standard" CT-ED curve to be used for all Halcyon linacs. However, extending this beyond the Pelvis preset iCBCT mode may introduce some uncertainty.

\section{References/Acknowledgements}

1. Jarema et al. Using the iterative $k V C B C T$ reconstruction on the Varian Halcyon linear accelerator for radiation therapy planning for pelvis patients Physica Medica 68 (2019) 112-116 


\section{P017 Auto segmentation of lung OARS using deep learning}

\author{
Akash Mehta, MAppSci ${ }^{1}$, Dr. Margot Lehman ${ }^{2}$, Senior Lecturer \\ Andrew Fielding ${ }^{3}$, A/Prof Prabhakar Ramachandran ${ }^{4}$ \\ ${ }^{1}$ Medical Physicist Registrar, Princess Alexandra Hospital, Brisbane, \\ Australia. (akash.mehta@health.qld.gov.au [Presenting author]). \\ ${ }^{2}$ Director of Radiation Oncology, Princess Alexandra Hospital, \\ Brisbane, Australia. (Margot.Lehman@ health.qld.gov.au). ${ }^{3}$ Master \\ of Medical Physics Course Coordinator, QUT, Brisbane, Australia. \\ (a.fielding@qut.edu.au). ${ }^{4}$ Director of Therapeutic Physics, Princess \\ Alexandra Hospital, Brisbane Australia. \\ (Prabhakar.Ramachandran@health.qld.gov.au)
}

Introduction The advancement in imaging modalities has led to the accumulation of a large amount of data which requires significant time for contouring and processing the data for clinical use. It can also lead to interobserver variations and human errors in delineating target and organs at risk (OARs) volumes. In this study, an effort has been made to develop an automatic segmentation tool and tested on lung OARs.

Method A deep learning-based model known as U-net architecture was used to develop and train a model to auto contour the OARs including the spine, heart, and both lungs. The model was trained for each OAR with 62 datasets for lungs using 100 epochs, the spine had 98 datasets and 23 patients for the heart with 200 and 100 epochs respectively. Upon training, the models were tested on datasets and their results were evaluated based on the dice similarity coefficient (DSC) and Hausdorff distance (HD).

Results The average DSC of left and right lung was found to 0.96 and 0.95 respectively whereas the spine produced 0.86 . With the lowest number of patients for heart, the model was able to yield the highest DSC of 0.97. In the case of evaluation using HD, the average distance between predicted and ground truth for the left lung was $3.0 \pm 0.8$ $\mathrm{mm}$ and was marginally higher for the right lung of $3.6 \pm 0.70 \mathrm{~mm}$. The lowest HD was observed in the spine, which was around $1 \mathrm{~mm}$, whereas the heart model yielded an average distance between both contours of $3.52 \pm 0.68 \mathrm{~mm}$.

Conclusion The model demonstrates the potential to be used in a clinical environment given that thousands of patient data were used in training. However, this study is still under investigation and more factors will be considered to make it more user friendly, reliable, and accurate for clinical purposes.

References/Acknowledgements

1. Forstner, D., \& Yap, M. (2015). Advances in radiation therapy. Medical Journal of Australia, 203(10), pp 394-395.

2. Razzak, M., Naz, S., \& Zaib, A. (2017). Deep Learning for Medical Image Processing: Overview, Challenges and the Future. Lecture Notes In Computational Vision And Biomechanics, pp 323-350.

3. Hesamian, M.H., Jia, W., He, X. et al. Deep Learning Techniques for Medical Image Segmentation: Achievements and Challenges. J Digit Imaging 32, pp 582-596 (2019).

4. Ronneberger O., Fischer P., Brox T. (2015) U-Net: Convolutional Networks for Biomedical Image Segmentation. In: Navab N., Hornegger J., Wells W., Frangi A. (eds) Medical Image Computing and Computer-Assisted Intervention - MICCAI 2015. MICCAI 2015. Lecture Notes in Computer Science, vol 9351. Springer, Cham
P018 Can we reduce imaging time and still generate acceptable Substitute CT for Prostate MRI Only Treatment Planning?

Tony Young ${ }_{4,7}^{1,2}$ Jason Dowling ${ }^{2,3,4}$, Robba Rai ${ }^{1,5}$, Gary Liney ${ }^{1,5,6}$, Peter Greer ${ }^{4,7}$, David Thwaites ${ }^{2}$, Lois Holloway ${ }^{1,2,5,6}$

${ }^{1}$ Liverpool and Macarthur Cancer Therapy Centres and Ingham Institute, Sydney, Australia. ${ }^{2}$ Institute of Medical Physics, School of Physics, University of Sydney, Sydney, Australia.

(Tony.Young@health.nsw.gov.au [Presenting author]). ${ }^{2}$ CSIRO Health and Biosecurity, The Australian e-Health \& Research Centre, Australia. ${ }^{3}$ South Western Sydney Clinical School, University of New South Wales, Australia. ${ }^{4}$ School of Mathematical and Physical Sciences, University of Newcastle, Australia.

(Jason.Dowling@csiro.au). ${ }^{5}$ University of New South Wales, Sydney, New South Wales, Australia. ${ }^{6}$ Centre for Medical Radiation Physics, University of Wollongong, Wollongong, New South Wales,

Australia. ${ }^{7}$ Calvary Mater Newcastle Hospital, Newcastle, New South Wales, Australia. (david.thwaites@sydney.edu.au),

(lois.holloway@health.nsw.gov.au)

Introduction The introduction of MRI linear accelerators (MR-linacs) requires improved approaches to MRI-only radiotherapy. MRI provides excellent soft tissue visualisation for target and organ definition, but no electron density information for dose calculation, obtained instead from registering CT images[1]. MRI-only radiotherapy would remove registration errors and reduce patient discomfort, workload and cost [2,3]. Electron density requirements may be addressed in different ways, from manually applying bulk density corrections $[2,4]$, to more computationally intensive methods, such as atlas based techniques [5] which automatically segment and apply bulk density corrections to produce substitute CT datasets (sCT). Reducing MRI imaging time would reduce potential artefacts from intrafraction motion and patient discomfort. This study investigated the effects of MRI imaging time reduction on $\mathrm{SCT}$ generation for prostate MRI-only treatment planning.

Method 10 volunteers were scanned on a Siemens Skyra 3T MRI. Sequences included the 3D T2-weighted (T2-w) SPACE used for SCT conversion as previously validated against CT [5], along with variations to this sequence in repetition time (TR), turbo factor, and combination of these to reduce the imaging time. Additionally, a T1 DIXON scan was taken to test the robustness of the sCT technique. All scans were converted to sCT and evaluated for anatomical changes and dosimetric differences for a standard VMAT plan compared to the previously validated SPACE sequence.

Results Compared to the previously validated T2-w SPACE sequence [5], scan times were reduced by up to $80 \%$. The external volume and bony anatomy were compared, with most meeting a DICE coefficient of 0.9 or better, with the largest variations occurring at the edges of the external body volume. Dosimetrically, most plans agreed within $2 \%$ at isocentre.

Conclusion This study demonstrates that MRI sequence imaging times can be reduced significantly with clinically acceptable sCT accuracy for dose calculation.

\section{References/Acknowledgements}

1. Nyholm T, Jonsson J. Counterpoint: Opportunities and Challenges of a Magnetic Resonance Imaging-Only Radiotherapy Work Flow. Seminars in Radiation Oncology. 2014;24:175-80. 
2. Karlsson M, Karlsson MG, Nyholm T, Amies C, Zackrisson B. Dedicated Magnetic Resonance Imaging in the Radiotherapy Clinic. International Journal of Radiation Oncology*Biology*Physics. 2009;74:644-51.

3. Roberson PL, McLaughlin PW, Narayana V, Troyer S, Hixson $\mathrm{GV}$, Kessler ML. Use and uncertainties of mutual information for computed tomography/magnetic resonance (CT/MR) registration post permanent implant of the prostate. Medical Physics. 2005;32:473-82.

4. Lambert J, Greer PB, Menk F, Patterson J, Parker J, Dahl K, et al. MRI-guided prostate radiation therapy planning: Investigation of dosimetric accuracy of MRI-based dose planning. Radiotherapy and Oncology. 2011;98:330-4.

5. Dowling JA, Sun J, Pichler P, Rivest-Hénault D, Ghose S, Richardson $\mathrm{H}$, et al. Automatic substitute computed tomography generation and contouring for magnetic resonance imaging (MRI)-Alone external beam radiation therapy from standard MRI sequences. International Journal of Radiation Oncology* Biology* Physics. 2015;93:1144-53.

\section{P019 Implementation of quality assurance procedure for deep inspiration breath hold (DIBH) treatments}

\section{A. Esposito, A. Gibbs}

Radiation Oncology Princess Alexandra Raymond Terrace (ROPART), South Brisbane, Australia. (alessandro.esposito@health.qld.gov.au [Presenting author]), (adrian.gibbs@health.qld.gov.au)

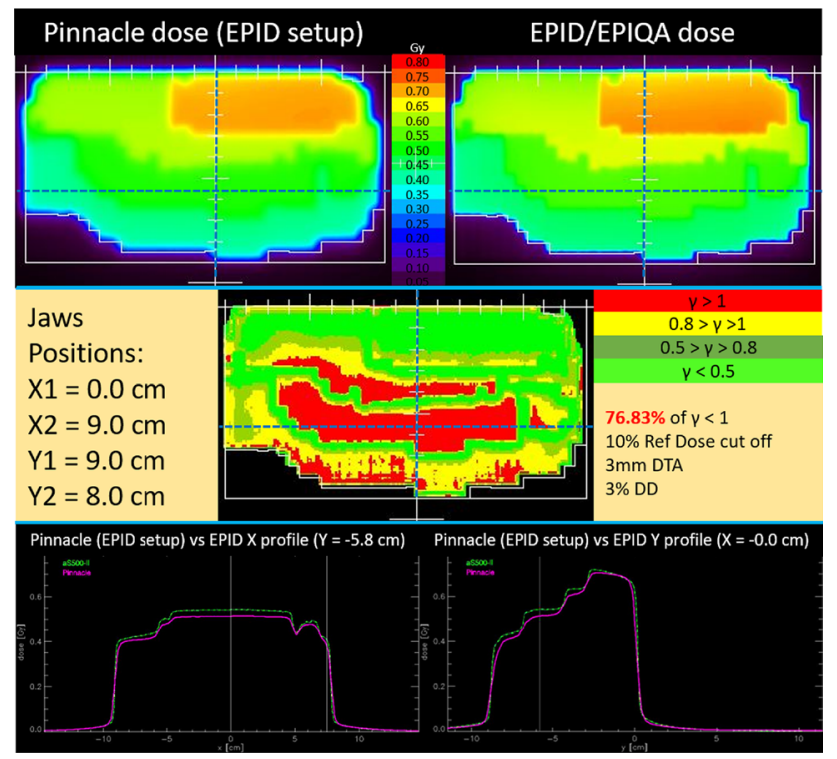

Figure 1 Top left, Pinnacle calculated dose (in EPID setup). Top right EPID measured dose. Gamma comparison in middle centre. Tick in axes represents $1 \mathrm{~cm}$. Bottom left, $\mathrm{X}$ profile comparison at $\mathrm{Y}=$ $-5.8 \mathrm{~cm}$, where gamma failed and bottom right $\mathrm{Y}$ profile comparison at $\mathrm{X}=0.0 \mathrm{~cm}$ (dashed blue lines in dose and gamma images)

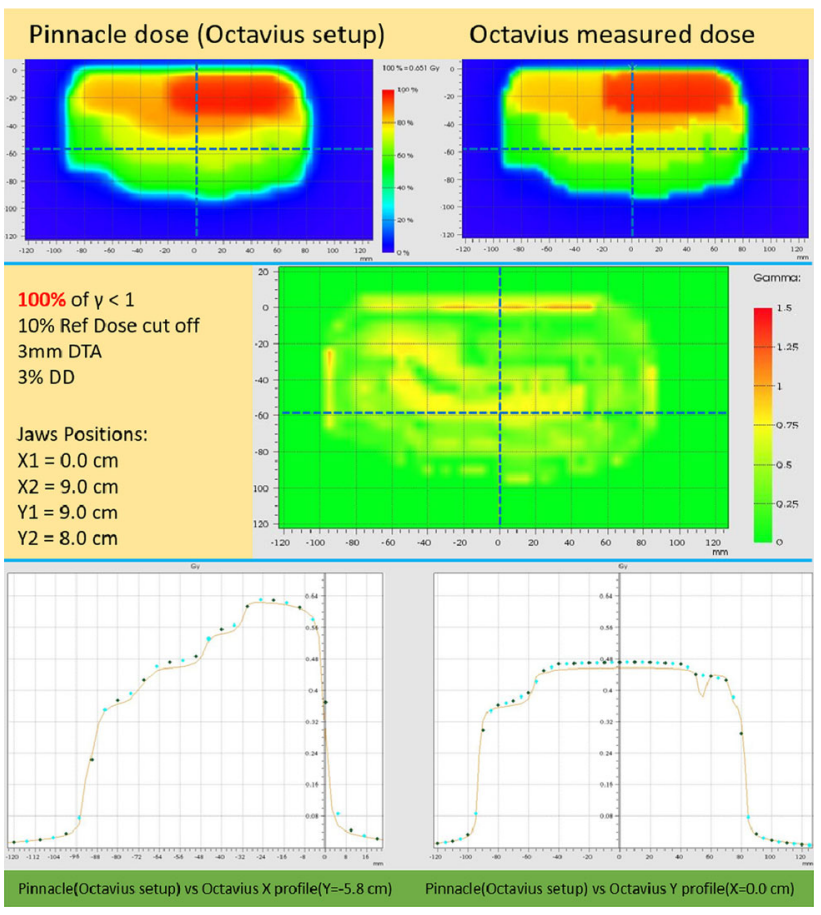

Figure 2 Top left, Pinnacle calculated dose (in Octavius setup). Top right Octavius measured dose. Gamma comparison in middle. Bottom left, $\mathrm{X}$ profile comparison at $\mathrm{Y}=-5.8 \mathrm{~cm}$ and bottom right $\mathrm{Y}$ profile comparison at $\mathrm{X}=0.0 \mathrm{~cm}$ (dashed blue lines in dose and gamma images). Profiles are at the same location as Fig. 1

Introduction Beams arrangement for DIBH technique involves two opposed tangential open and modulated fields. Lung dose is minimised by using one zero-jaw on collimator axis. The current DIBH physics QA procedure at ROPART involves point dose verification using measured data. A fluence based QA was sought. Two options were considered:

1. Varian Electronic Portal Image Dosimetry (EPID) and

2. Ion chamber array (Octavius PTW)

Both Octavius and Pinnacle TPS had been commissioned for zero-jaw asymmetric fields using water phantom scans and field output measurements. Water phantom scans in DIBH asymmetric conditions were not available for EPID commissioning purposes at the specific depth $(1.5 \mathrm{~cm})$ and SSD $(98.5 \mathrm{~cm})$.

Method EPID in conjunction with EPIQA software based on the GLAaS algorithm ${ }^{(1)}$ and the Octavius chamber array together with PTW Verisoft software allows for Gamma analysis QA.

TPS fluence maps were compared with both the EPID/EPIQA and Octavius/Verisoft using the Gamma Function (3\%/v), with a pass/fail rate acceptance of $90 \%$.

While the EPID measurement depth is fixed at $1.5 \mathrm{~cm}, 3.7 \mathrm{~cm}$ measurement depth was chosen for the Octavius to maximize the 
signal for both $6 \mathrm{X}$ and $10 \mathrm{X}\left(\mathrm{d}_{\max } 1.5\right.$ and $\left.2.2 \mathrm{~cm}\right)$ and avoid the buildup region.

Results One example of EPID vs Pinnacle for a modulated field is shown in Figure 1 . Gamma $3 \% / 3 \mathrm{~mm}$ resulted in $76.83 \%$ of points $<$ 1. The profile comparison shows differences in absolute dose. The open field at same angle resulted in $79.44 \%$ gamma points $<1$.

Using Octavius, $100.0 \%$ of gamma points were $<1$ (Figure 2) with same criteria for all the open and modulated beams.

Conclusion The EPID/EPIQA is not accurate to provide pass rates greater than $90 \%$, whereas the results for the Octavius system demonstrate it is a reliable tool for DIBH QA. The inaccuracies of the EPID system will be discussed.

\section{References/Acknowledgements}

1. Nicolini, G., Fogliata, A., Vanetti, E., Clivio, A. and Cozzi, L. (2006), GLAaS: An absolute dose calibration algorithm for an amorphous silicon portal imager. Applications to IMRT verifications. Med. Phys., 33: 2839-2851. https://doi.org/10.1118/ 1.2218314

\section{P020 Long term stability of AlignRT-MV congruence calibration}

\section{N Heffernan, L Yuen, M Holmes, M West}

GenesisCare, Queensland, Australia.

(David.Heffernan@genesiscare.com (Presenting author),

(Lockie.Yuen@genesiscare.com), (Mark.Holmes@genesiscare.com), (Mark.West@genesiscare.com)

Introduction AlignRT is a surface-guided radiation therapy (SGRT) technology that can monitor inter- and intra-fraction patient motion. Congruence of the SGRT and MV isocentres ("isocentre calibration") is vital to prevent artificial walkout caused by rotation about different isocentres [1]. This is particularly true when using non-coplanar beams. Despite this, there is little research discussing the stability of isocentre calibration or suggestions for QA tolerances.

Since installing AlignRT at GenesisCare Southport we have performed pre-treatment QA of the AlignRT system for each singlefraction stereotactic procedure. These measurements provide

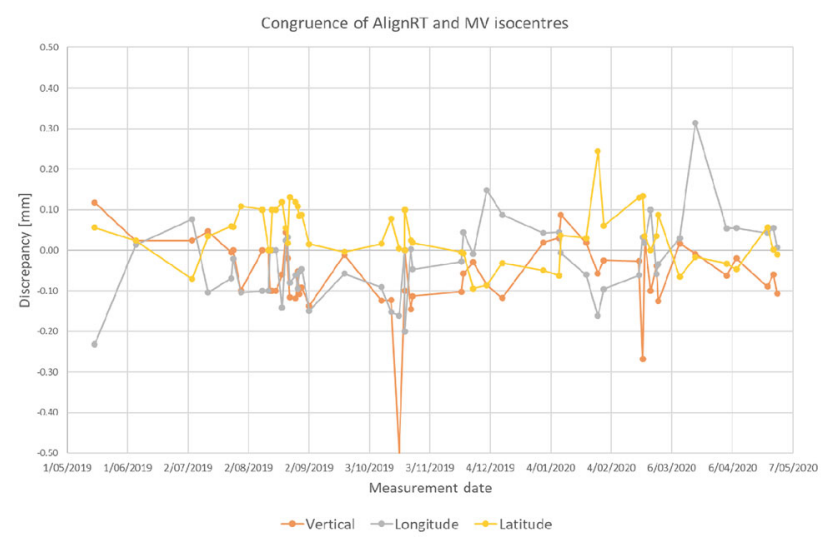

Figure 1 Congruence of AlignRT and MV isocentres over time information on the long-term stability and accuracy of the isocentre calibration.

Method We performed pre-treatment QA using the AlignRT calibration cube in accordance with manufacturer recommendations [2]. AlignRT saves the isocentre calibration results as a report in PDF and XML formats. For this work we manually inspected the XML reports and copied the relevant information to a spreadsheet for analysis.

Results We performed analysis on 53 measurements taken over 12 months from May 2019 to April 2020. Average discrepancies between the AlignRT and MV isocentres ranged from $-0.06 \mathrm{~mm}$ to $+0.04 \mathrm{~mm}$ in the three translational directions. The maximum standard deviation was $0.09 \mathrm{~mm}$. Rotational discrepancies had a maximum standard deviation of 0.06 degrees.

We plot the congruence of AlignRT-MV isocentres over time in Figure 1. No long term trends are visible.

Conclusion We performed an analysis of pre-treatment SGRT QA results. The results show that the AlignRT-MV congruence calibration is stable and accurate. We suggest reducing the frequency of isocentre calibration QA to monthly. From the results of the analysis we suggest an action level of $0.2 \mathrm{~mm}$ or 0.2 degrees for the isocentre calibration.

References

1. Li G (2020) Commissioning and Routine Quality Assurance of the Vision RT AlignRT ${ }^{\circledR}$ System. In: Hoisak J (ed) Surface Guided Radiation Therapy, 1st edn. CRC Press, pp 157-181.

2. VisionRT (2019) Vision RT Calibration Phantom User Guide, issue 3.2 .

\section{P021 Review of ion chamber array for photon beam quality index and electron energy measurements}

\section{Seshadri, S Ibrahim, P Ramachandran}

Radiation Oncology, Princess Alexandra Hospital, Ipswich Road, Australia. (Venkatakrishnan.Seshadri@health.qld.gov.au), (salma.ibrahim@health.qld.gov.au), (Prabhakar.Ramachandran@ health.qld.gov.au)

Introduction Beam quality index (BQI) verification is part of the recommended periodic QA tests by the AAPM and IPEM. Common implementation from vendors utilise a set of wedges to determine the beam energy. Another common method is to use ratios of ionisation at two depths to verify beam quality constancy, which is time consuming. This study compares wedge implementations by Sun Nuclear Inc (Quad Wedge) and PTW Dosimetry (BQ - Check) for verifying constancy of BQI of photon beams and energy of electron beams.

Method Quad-wedge phantoms were used with SNC IC Profiler array and SNC Profiler software. The BQ Check phantom was used with PTW Starcheck array and PTW Multicheck software. Copper and aluminium wedges are used for photon and electron beam measurements respectively. SNC quad-wedges requires a $30 \mathrm{~cm} \times 30 \mathrm{~cm}$ field for use with photons and a $25 \mathrm{~cm}$ applicator for use with electrons. The PTW BQ check cannot be used with FFF beams. The measurements were compared against reference PDD data measured with water-tank and PTW Roos chamber on the same day.

Results Deviation in energy measurements compared to water-tank measurements are given below. The largest deviation was $1 \mathrm{~mm}$ in R50 for both PTW and SNC. Photon beam quality was within $0.5 \%$ and $0.3 \%$ for PTW and SNC respectively. 


\begin{tabular}{|c|c|c|c|c|c|}
\hline \multirow[t]{2}{*}{$\begin{array}{l}\text { Electron } \\
\text { Energy }\end{array}$} & \multicolumn{2}{|c|}{$\begin{array}{l}\text { Difference in R50 } \\
\text { from water-tank data }\end{array}$} & \multirow[t]{2}{*}{$\begin{array}{l}\text { Photon } \\
\text { Energy }\end{array}$} & \multicolumn{2}{|c|}{$\begin{array}{l}\text { Difference from water- } \\
\text { tank data }\end{array}$} \\
\hline & $\begin{array}{l}\text { PTW } \\
\text { BQ } \\
\text { Check }\end{array}$ & $\begin{array}{l}\text { SNC } \\
\text { Quadwedges }\end{array}$ & & $\begin{array}{l}\text { PTW } \\
\text { BQ Check } \\
\left(\mathrm{TPR}_{20,10}\right)\end{array}$ & $\begin{array}{l}\text { SNC } \\
\text { Quadwedges } \\
\text { (D10) }\end{array}$ \\
\hline $06 \mathrm{MeV}$ & $0.3 \mathrm{~mm}$ & $1.0 \mathrm{~mm}$ & $06 \mathrm{MV}$ & $+0.42 \%$ & $+0.10 \%$ \\
\hline $09 \mathrm{MeV}$ & $0.9 \mathrm{~mm}$ & $-0.1 \mathrm{~mm}$ & $10 \mathrm{MV}$ & $+0.47 \%$ & $+0.23 \%$ \\
\hline $12 \mathrm{MeV}$ & $\begin{array}{l}-0.3 \\
\mathrm{~mm}\end{array}$ & $-0.9 \mathrm{~mm}$ & $\begin{array}{l}06 \mathrm{MV} \\
\mathrm{FFF}\end{array}$ & N.A & $-0.06 \%$ \\
\hline $15 \mathrm{MeV}$ & $1.0 \mathrm{~mm}$ & $0.2 \mathrm{~mm}$ & $\begin{array}{l}10 \mathrm{MV} \\
\text { FFF }\end{array}$ & N.A & $+0.03 \%$ \\
\hline
\end{tabular}

An induced error of $1 \mathrm{~mm}$ in inline and crossline directions in placement of the wedges on the array induces an additional error of up to $2 \mathrm{~mm}$ in electron energy measurements using SNC quad-wedges and up to $1 \mathrm{~mm}$ in PTW BQ Check.

Conclusion Preliminary results indicate both arrays provide comparable output when proper setup conditions are met. However, PTW BQ - Check seems to handle misalignment in setup of the wedge. Measurements will be undertaken at regular periods to validate the use of the wedge phantoms to check beam energy over time.

\section{P022 Calibration of the Leksell Gamma Knife (LGK) Solid Water Phantom (SWP) hole-punch location for patient positioning system (PPS) versus radiation focal position (RFP) isocentre coincidence QA}

\section{E Jones, B Perrett}

Princess Alexandra Hospital, Brisbane, Australia. (catherine.jones3@health.qld.gov.au [Presenting author]), (ben.perrett@health.qld.gov.au)

Introduction Geometrically accurate delivery of LGK radiotherapy treatments is dependent on precise alignment of the PPS with the RFP. QA of PPS-RFP coincidence is performed with an Elekta diode tool and a film holder tool. Elekta also provides a SWP which includes a film insert. Film is firmly immobilized in the phantom with two rods inserted in holes generated with a specialized hole punch. Here we investigate the reproducibility of these hole-punch alignment marker positions, with a view to generating a third independent PPS-

Table 1 PPS and RFS isocentre measurements with axially-orientated EBT3 film in the LGK SWP

\begin{tabular}{|c|c|c|c|c|c|c|c|c|}
\hline \multirow[t]{3}{*}{$\begin{array}{l}\text { Colli- } \\
\text { mater }\end{array}$} & \multicolumn{4}{|c|}{$\begin{array}{l}\text { Measured uncorrected-PPS } \\
\text { vs RFS shift }(\mathrm{mm})\end{array}$} & \multicolumn{2}{|c|}{$\begin{array}{l}\text { Film PPS-cor- } \\
\text { rection shift } \\
(\mathrm{mm})\end{array}$} & \multicolumn{2}{|c|}{$\begin{array}{l}\text { Difference } \\
\text { within film } \\
\text { pairs (mm) }\end{array}$} \\
\hline & \multicolumn{2}{|c|}{ Left-Right } & \multicolumn{2}{|c|}{ Ant-Post } & \multirow{2}{*}{$\begin{array}{l}\text { Left- } \\
\text { Right }\end{array}$} & \multirow{2}{*}{$\begin{array}{l}\text { Ant- } \\
\text { Post }\end{array}$} & \multirow{2}{*}{$\begin{array}{l}\text { Left- } \\
\text { Right }\end{array}$} & \multirow{2}{*}{$\begin{array}{l}\text { Ant- } \\
\text { Post }\end{array}$} \\
\hline & $\begin{array}{l}\text { Film } \\
1\end{array}$ & $\begin{array}{l}\text { Film } \\
2\end{array}$ & $\begin{array}{l}\text { Fil } \\
1\end{array}$ & 2 & & & & \\
\hline पाIII & 0. & 0.2 & -4 & 7 & & $-t$ & & 0.0 \\
\hline $\mathrm{m}$ & 0. & 0.183 & -0.256 & -0.241 & 0. & -0.249 & 0. & 0.0 \\
\hline $6 \mathrm{~m}$ & 0.175 & 0.174 & -0.211 & -0.190 & 0.175 & -0.201 & 0.001 & 0.02 \\
\hline
\end{tabular}

RFP coincidence test, as well as an efficient and accurate tool for aligning film-measured and TPS-calculated dose-maps.

Method QA following a recent service included acquisition of two SWP irradiations each of the $4 \mathrm{~mm}, 8 \mathrm{~mm}$, and $16 \mathrm{~mm}$ collimators with EBT3-film aligned in the axial plane. The digitized (400 dpi) films were analysed for reproducibility of the hole-punch-measured PPS isocentre within the pairs of film, and the difference in measured RFP of the three collimator irradiations.

Analysis was performed in Python. The centre of each hole was measured by generating a binary-image using a global threshold of the blue-channel image, followed by morphological-closing and opening to remove noise. A region-growing-algorithm separated the hole-regions. PPS isocentre was calculated as the mean pixel position of the two-hole-regions. RFP was similarly measured.

Results As summarized in Table 1, the measured PPS-RFS was reproducible within pairs of axial-digitized-films (max discrepancy of $0.021 \mathrm{~mm}$ ). The mean shift for each pair (column "Film PPS-correction" in Table 1) provides the shift to be implemented when performing PPS-vs-RFP QA using this method.

Conclusion Although more repeat-measurements (including of coronal-oriented film) are required to verify these results, an additional PPS-vs-RPS coincidence QA-method has been developed.

\section{P023 Evaluation of Machine Performance Check system on Varian Halcyon linear accelerator}

\author{
A Kazi ${ }^{1}$, T Jarema ${ }^{1}$, D Whaites ${ }^{2}$ \\ ${ }^{1}$ Icon Cancer Centres, Toowoomba, Australia. \\ (aleksandra.kazi@icon.team [Presenting author]), \\ (talia.jarema@icon.team). ${ }^{2}$ Icon Cancer Centres, Northlakes, \\ Australia. (diana.whaites@icon.team)
}

Introduction The machine performance check (MPC) is an integrated quality assurance (QA) tool on a Varian Halcyon linear accelerator (Halcyon). The aim of this study was to evaluate its suitability and reliability for daily output and uniformity checks.

Method Measurements were done on a Halcyon located at the Icon Cancer Centre, Toowoomba, over 28 months, for the 6 MV flattening filter free photons (6 MV FFF) beam. During this time the Halcyon has undergone an upgrade to Version 2.0.

MPC uses the existing megavoltage electronic portal imaging device (EPID) to assess the beam output and beam uniformity variation. The MPC uniformity measurement variations are given as a single result that comprises both vertical and horizontal planes.

MPC checks were compared against the daily checks acquired with the Sun Nuclear Daily QA3 device, as well as the monthly checks using PTW Farmer ionization chamber (IC) for output measurements and a PTW Octavius 1500 detector array (1405 planeparallel vented ionization chambers) for symmetry checks.

Results The MPC-measured beam output drifted by $1.46 \%$ per year comparing to the monthly Farmer IC and Daily QA3 measurements, taking an average of 4 months to change by $0.5 \%$.

MPC uniformity results were within $1.0 \%$ from the monthly, and within $1.5 \%$ from the daily symmetry measurements for Halcyon Version 1. Following upgrade to Version 2, MPC uniformity was within $0.5 \%$ and $1.0 \%$ from the monthly and daily symmetry assessment methods, respectively.

Conclusion MPC on Halcyon was verified as a daily QA tool for Halcyon beam output and symmetry against independent detectors over 28 months. Trends show it's reliable and stable, observed drift in MPC-measured output was slow in comparison with the frequency of QA using the Farmer IC. The absolute difference is subject to the reference values acquired. 
P024 The use of a Monte Carlo system for regular comparison with patient specific $\mathbf{Q A}$ measurements and TPS calculations for SBRT treatments.

\author{
Tim S Markwell ${ }^{1}$, Alexander Livingstone ${ }^{2}$ \\ ${ }^{1}$ ROPART - Radiation Oncology Princess Alexandra Hospital \\ Raymond Terrace, Brisbane, Australia. \\ (Tim.Markwell@health.qld.gov.au [Presenting author]). ${ }^{2}$ RBWH - \\ Royal Brisbane and Women's Hospital. \\ (Alexander.Livingstone@ @ealth.qld.gov.au)
}

Introduction Patient specific QA (PSQA) is an important part of the treatment pathway to ensure that the treatments delivered to the patient match what is calculated in the planning system. For Stereotactic Body Radiation Therapy (SBRT) type treatments, small fields and high levels of modulation can create larger discrepancies between measurements and planning systems. Monte Carlo (MC) offers a third gold-standard system to help determine if the differences are due to the planning system calculations, or the machine delivery. This investigation has implemented a Monte Carlo system using EGSnrc/ BEAMnrc/DOSXYZnrc [1], MCtools [2] and custom in house code to allow the regular use of MC simulations to be compared with measured and calculated data.

Method VMAT SBRT Arcs were simulated using BEAMnrc in the PSQA Geometry (dMax, $98.5 \mathrm{~cm}$ SSD) used by EPIQA to allow comparison against the Pinnacle treatment planning system (Philips, Netherlands) and measured EPID data. EPIQA (Epidos, Slovakia) uses an integrated EPID image acquired during the arc which is then converted to dose using the GlAaS Algorithm [3]. MC simulations were performed to a custom water phantom that matched the Pinnacle configuration, allowing the export and comparison of DICOM dose data in the EPIQA software. Brain and Lung treatments were simulated with a total of 19 arcs simulated and compared with measurements and Pinnacle calculations.

Results Simulations took in the order of 3-5 minutes per arc to achieve an uncertainty of $<0.4 \%$ on a dedicated compute cluster with 192 threads. For the Brain treatments all arcs passed a gamma analysis at $2 \%, 2 \mathrm{~mm}>90 \%$, with a slightly better agreement between MC results and measured data compared than Pinnacle Vs Measured data. For the Lung areas, there was a larger discrepancy with MC Vs Measured, with most arcs passing at 3\%, $2 \mathrm{~mm}>90 \%$.

Conclusion A MC system has been implemented to allow direct comparison with measured PSQA data and TPS calculations. A good agreement was achieved across all systems, with slightly larger discrepancies evident with lung type arcs.

\section{References/Acknowledgements}

1. Rogers, D.W.O., Faddegon, B.A., Ding, G.X., Ma, C.-M., We, J. and Mackie, T.R. (1995), BEAM: A Monte Carlo code to simulate radiotherapy treatment units. Med. Phys., 22: 503-524. https://doi.org/10.1118/1.597552

2. MC Tools : Livingstone A : https://github.com/livingag/MCtools

3. Nicolini G. et al: GLaS: An absolute dose calibration algorithm for an amorphous silicon portal imager. Applications to IMRT verifications, Med. Phys. 33, 2839 (2006)
P025 Development of a CT number calibration audit phantom and the tolerance levels for each tissue type

Minoru Nakao ${ }^{1,2}$, Masahiro Hayata ${ }^{1}$, Shuichi Ozawa ${ }^{1,2}$, Hideharu Miura $^{1,2}$, Kiyoshi Yamada ${ }^{1}$, Kosaku Habara ${ }^{1}$, Fumio Okamura ${ }^{1}$, Daisuke Kawahara ${ }^{1}$, Kentaro $\mathrm{Miki}^{3}$, Takeo Nakashima ${ }^{3}$, Yusuke Ochi $^{3}$, Shintaro Tsuda ${ }^{3}$, Mineaki Seido ${ }^{4}$, Yoshiharu Morimoto ${ }^{4}$, Atsushi Kawakubo ${ }^{5}$, Hiroshige Nozaki ${ }^{6}$, Hirofumi Fukunaga ${ }^{7}$, Yoshimasa $\operatorname{Toyota}^{7}$, Yasushi Nagata ${ }^{1,2}$

${ }^{1}$ Hiroshima High-Precision Radiotherapy Cancer Center, Hiroshima, Japan. (nakao@hiprac.jp [Presenting author]). (hayata@hiprac.jp), (ozawa@hiprac.jp), (miura@hiprac.jp), (yamada@hiprac.jp), (habara@hiprac.jp), (okamura@hiprac.jp). ${ }^{2}$ Hiroshima University, Hiroshima, Japan. (daika99@ hiroshima-u.ac.jp). ${ }^{3}$ Hiroshima University Hospital, Hiroshima, Japan. (kentaro-miki@ hiroshimau.ac.jp), (mla@hiroshima-u.ac.jp), (ysk@hiroshima-u.ac.jp), (kawashin@hiroshima-u.ac.jp), (nagat@hiroshima-u.ac.jp). ${ }^{4}$ Hiroshima Prefectural Hospital, Hiroshima, Japan. (saydo@vesta.dti.ne.jp), (rt.ymorimoto@gmail.com). ${ }^{5}$ Hiroshima City Hiroshima Citizens Hospital, Hiroshima, Japan. (kawakubo6405211@gmail.com). ${ }^{6}$ Hiroshima Red Cross Hospital \& Atomic-bomb Survivors Hospital, Hiroshima, Japan. (hnozaki@hiroshima-med.jrc.or.jp). ${ }^{7}$ Hiroshima Prefecture Cancer Control Division. (h-fukunaga84794@pref.hiroshima.lg.jp), (ytoyota81786@pref.hiroshima.lg.jp)

Introduction In photon radiation therapy, computed tomography (CT) numbers are converted into mass densities (MDs) or relative electron densities to water (REDs) for dose calculation. In order to validate CT-MD and CT-RED calibration tables registered in the radiotherapy treatment planning systems, a postal audit phantom and a stoichiometric CT calibration with three-parameter fit model have

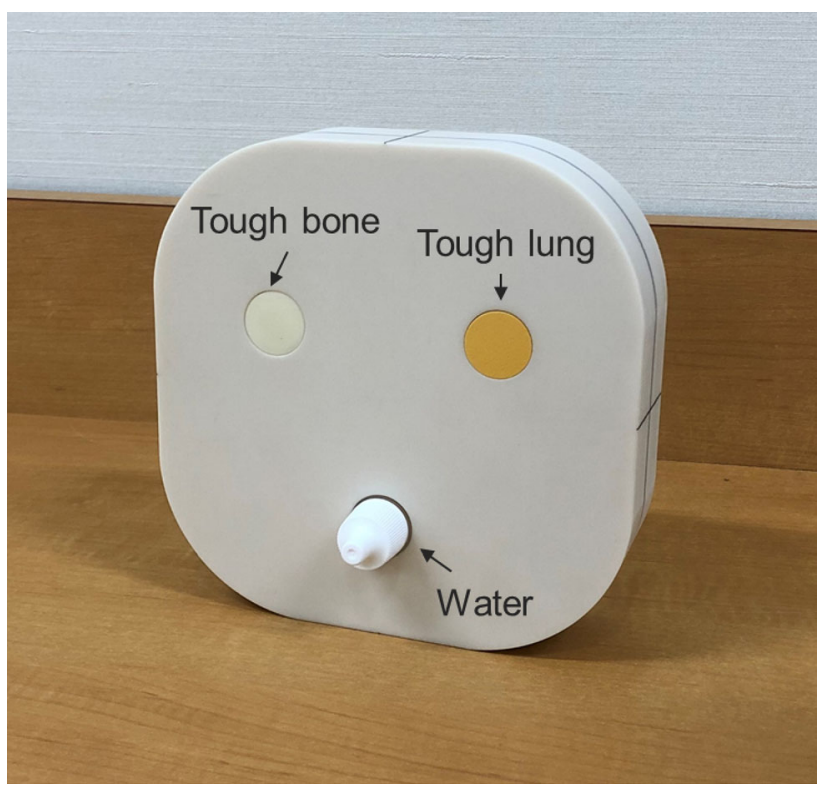

Figure 1 A postal audit phantom 

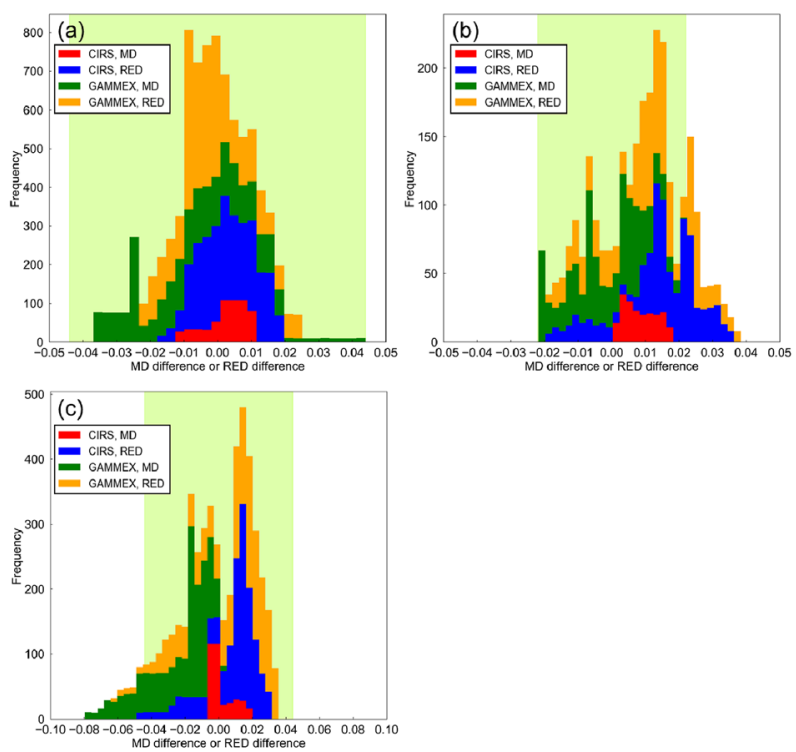

Figure 2 (a) Lung. (b) Adipose/muscle. (c) Cartilage/spongy-bone

been developed [1]. The purpose of our study is to demonstrate an outline of the CT number calibration audit through a multi-institutional study.

Method A postal audit phantom was developed with tough lung and tough bone inserts (Kyoto Kagaku, Kyoto, Japan) (Fig. 1). And, a new stoichiometric CT calibration with empirical three-parameter fit was established. As a pilot study for CT number calibration audit, the postal audit phantom was scanned by six CT scanners at five radiotherapy institutions. The scan conditions were the same as those of the treatment planning CT scan. The obtained CT-MD and CT-RED calibration tables were compared to the CT number calibration tables registered in a radiation treatment planning system (RTPS) at each radiotherapy institutions. The MD or RED differences were compared to the tolerance level, which caused a $2 \%$ dose difference. Results The MD or RED differences for each tissue type are shown in Fig. 2 (a)-(c) as stacked histograms. The tolerance levels of lung, adipose/muscle and cartilage/spongy-bone were $0 \pm 0.044,0 \pm 0.022$ and $0 \pm 0.044$, respectively [2-3]. A part of the MD or RED difference for adipose/muscle and cartilage/spongy-bone were exceeded the tolerance levels. However, the mean values of the MD or RED difference were not exceeded the tolerance levels.

Conclusion We developed a CT number calibration phantom for a postal audit and demonstrated a flow of the CT number calibration audit at five radiotherapy institutions.

\section{References}

1. Nakao M, Ozawa S, Miura H, Yamada K, Habara K, Hayata M et al (2020) Development of a CT number calibration audit phantom in photon radiation therapy: A pilot study. Med Phys, 47(4):1509-1522

2. Nakao M, Ozawa S, Yamada K, Yogo K, Hosono F, Hayata M et al (2018) Tolerance levels of CT number to electron density table for photon beam in radiotherapy treatment planning system. J Appl Clin Med Phys, 19(1):271-275,

3. Nakao M, Ozawa S, Yogo K, Miura H, Yamada K, Hosono F et al (2019) Tolerance levels of mass density for CT number calibration in photon radiation therapy. J Appl Clin Med Phys, 20(6):45-52

\section{P026 3D printed Winston Lutz phantom for LINAC quality assurance}

\section{E. Simpson-Page ${ }^{1}$, A. Livingstone ${ }^{1}$, S. Peet ${ }^{1}$, S. B. Crowe ${ }^{1,2}$}

${ }^{1}$ Cancer Care Services, Royal Brisbane and Women's Hospital, Brisbane. (Emily.simpson-page@health.qld.gov.au [Presenting Author]), (Alexander.livingstone@ health.qld.gov.au), (Samuel.peet@health.qld.gov.au). ${ }^{2}$ Queensland University of Technology, Brisbane, Queensland, Australia. (Scott.crowe@health.qld.gov.au)

Introduction In stereotactic body radiosurgery it is imperative that the isocentre of the linear accelerator is well characterised. The isocentre should be the intersection between the mechanical axis of the gantry, treatment couch and collimator with the imaging isocentre and treatment beam axis [1]. This can be achieved with a Winston Lutz test, wherein a phantom containing a small radiopaque marker is imaged at a series of gantry, couch and collimator positions.

Method A Winston Lutz phantom was created in house at the Royal Brisbane and Women's Hospital on Raise 3D Pro 2 dual extrusion printer, using both standard $1.75 \mathrm{~mm}$ PLA filament and $1.75 \mathrm{~mm}$ Copperfill plastic filament to achieve contrast between ball bearing and outer cube [2]. A Copperfill sphere was printed inside a PLA cube with indents for alignment and optimised ooze conditions to reduce artefacts. Isocentre analysis was achievable using 8 LINAC orientations; 4 Gantry Angles at 270, 0, 90 and 180, collimator angles at 270

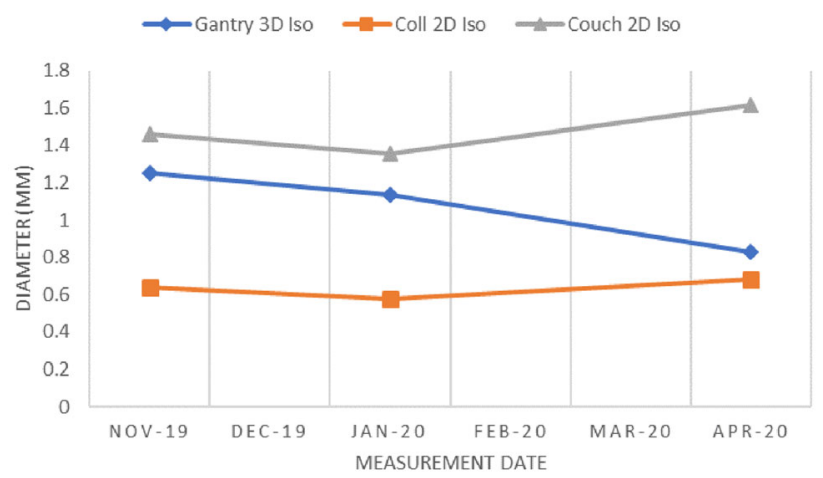

Figure 1 Isocentre measurements taken over a period of six months

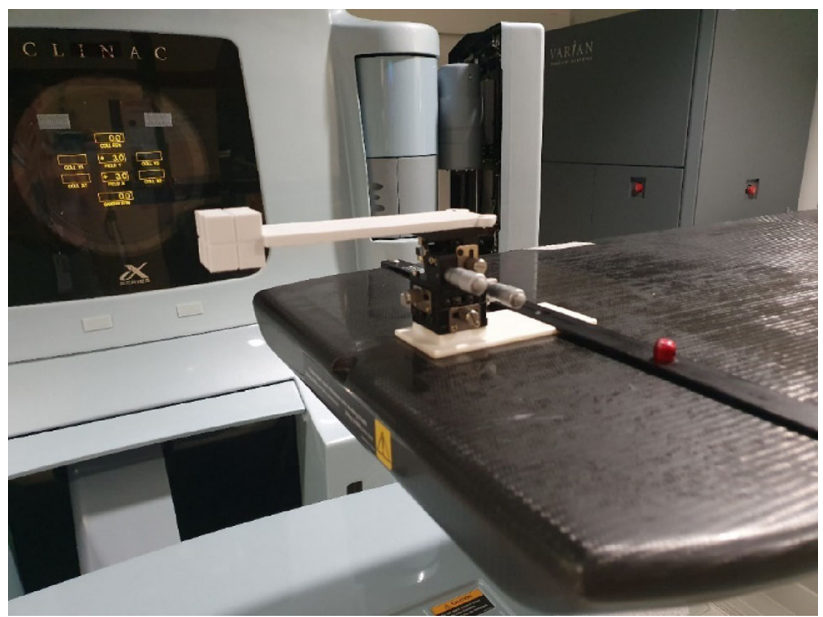

Figure 2 Fabricated Winston Lutz Phantom 
and 90 degrees, and two couch angles at 270 degrees and 90 degrees [3]. The phantom was mounted on an xyz stage and fixed in place with an indexing bar. PyLinac Winston Lutz algorithm was used to analyse the images taken at each orientation.

Results The phantom was successfully created for routine quality assurance locally. The 8 LINAC orientations chosen provided enough information for the PyLinac Winston Lutz algorithm to produce information about the isocentre of the LINAC. Tracking progress with QAtrack + , over a period of six months, the Gantry 3D isocentre varied by less than $0.4 \mathrm{~mm}$ and the Collimator $2 \mathrm{D}$ isocentre and Couch 2D isocentre varied by less than $0.11 \mathrm{~mm}$ and $0.3 \mathrm{~mm}$ respectively (Figs. 81, 82).

Conclusion This in house design provides an affordable, easily implemented option for a reproducible Winston Lutz phantom to provide quality assurance on linear accelerator isocentres.

\section{References}

1. T. Denton, L. Shields, J. Howe and A. Spalding, "Quantifying isocenter measurements to establish clinically meaningful thresholds," Journal of Applied Clinical Medical Physics, vol. 16, no. 2, pp. 175-188, 2014.

2. O. Dancewicz, S. Sylvander, T. Markwell, S. Crowe and J. Trapp, "Radiological properties of 3D printed materials in kilovoltage and megavoltage photon beams," European Journal of Medical Physics, vol. 38, pp. 111-118, 2017.

3. W. Du, J. Johnson, W. Jiang and R. Kudchadker, "On the selection of gantry and collimator angles for isocenter localization using Winston-Lutz tests," Journal of Applied Clinical Medical Physics, pp. 167-178, 2016.

\section{P027 Patient specific QA with perfraction- troubleshooting experience for a smooth run}

\author{
Jaysree Ukath ${ }^{1}$, Penny Fogg ${ }^{1}$, Brindha Subramanian', Matthew \\ Murray $^{2}$ \\ ${ }^{1}$ Medical Physics Specialist, Genesiscare, QLD. \\ (Jaysree.Ukath@genesiscare.com), (Penny.Fogg@genesiscare.com), \\ (Brindha.Subramanian@genesiscare.com). ${ }^{2}$ Director, Medical \\ Physics, Genesiscare, QLD. (Matthew.Murray@genesiscare.com)
}

Introduction The Fraction-0 module of PerFraction ${ }^{\mathrm{TM}}$ (PF) from Sun Nuclear Corporation (SNC) is used as pre-treatment quality assurance(QA) tool in our practice. PF automatically compares the in-air plan delivery of calibrated electronic portal imaging device (EPID) with the predicted plan dose. The calibration method to convert the images to absolute dose is from SNC and establishes EPID response to dose correction factor tables for each: pixel position, field width and primary to scatter contribution along with reference dose information. For PF to perform, monitoring EPID performance is crucial as issues with EPID in addition to the plan delivery systems can result in QA failure. The aim of this study was to follow-up few PF QA failures and identify the necessity for alteration of test frequencies, based on clinic specific experience.

Method Different instances of PerFraction IMRT and VMAT QA fails on Varian 21iX and TrueBeam (TB) were investigated, causes for the failures identified and preventive actions discussed.

Results Though both $21 \mathrm{iX}$ and TB has aS1000 panels, 21iX panel nearing end of its life, indicated frequent failures. Failed cases on this $21 \mathrm{iX}$ passed a second exposure on another beam matched linac indicating issues with EPID performance. Since output drifts can change flood field (FF) corrections for an aging panel, a repetition of the dark(DF) and FF resulted in QA pass. This test frequency is now changed to biweekly from monthly. A different set of QA failures revealed that the FF for the linac panel was delivered at a non-zero collimator angle. Repeat delivery of DF and FF at correct collimator angle resolved this. A set of TrueBeam QA failures was resolved by repeating an isocal verification and subsequent calibration. Routine monthly checks being within tolerance, the only parameter to be verified was imager centre drift. Another group of failures on TrueBeam required EPID absolute dose calibration. Panel service/ mechanical recalibrations, or output drifts can lead to a change in the SNC stored correction factors. The calibration frequency is altered to biannual from annual on this linac.

Conclusion PerFraction ${ }^{\mathrm{TM}}$, a simplified solution for patient specific QA can become time consuming process for Medical physicist facing unexplained QA failures, from unexpected changes in EPID performance. A streamlined QA programme based on TG142 combining TG 100 guidelines to address factors impairing performance quality with increased test frequency can lead to a smooth patient specific QA solution.

\section{References/Acknowledgements}

1. Eric E. Klein, Joseph Hanley, John Bayouth, Fang-Fang Yin, William Simon Sean Dresser, Christopher Serago, Francisco Aguirre, Lijun Ma, Bijan Arjomandy, Chihray Liu, Carlos Sandin, Todd Holmes (2009) Task Group 142 report: Quality assurance of medical accelerators. Med Phys 36 (9):4197-4212

2. M Saiful Huq, Benedick A Fraass, Peter B Dunscombe, John P Gibbons Jr, Geoffrey S Ibbott, Arno J Mundt, Sasa Mutic, Jatinder R Palta, Frank Rath, Bruce R Thomadsen, Jeffrey F Williamson, Ellen D Yorke (2016) The Report of Task Group 100 of the AAPM: Application of Risk Analysis Methods to Radiation Therapy Quality Management. Med. Phys. 43 (7), 4209-4262

\section{P028 PerFRACTION fraction $0^{\mathrm{TM}}$ 2D analysis commissioning across three Elekta agility machines}

\section{S F Liu, L Webb, V Seshadri, M Wawrzak, P Hanlon, P Ramachandran}

Radiation Oncology, Princess Alexandra Hospital, Ipswich Road, Australia. (SauFan.Liu@health.qld.gov.au),

(Luke.Webb@health.qld.gov.au),

(Venkatakrishnan.Seshadri@health.qld.gov.au),

(Michal.Wawrzak@health.qld.gov.au),

(Peta.Hanlon@health.qld.gov.au),

(Prabhakar.Ramachandran@health.qld.gov.au)

Introduction PerFRACTION Fraction $0^{\mathrm{TM}}$ collects the patient plan data delivered to the EPID, allowing dose reconstruction to be compared via gamma analysis against the TPS dosimetry, prior to the patient treatment. PerFRACTION measurements were performed on three licenced Elekta Agility machines, PA2/PA5/PA6, to investigate panel response and PerFRACTION model accuracy.

Method Elekta linear accelerators have a fixed Source-to-Image Distance (SID) of $160 \mathrm{~cm}$ with $1024 \times 1024$ pixels split across 16 subsections, covering the panel size of $41 \mathrm{~cm} \times 41 \mathrm{~cm}$, giving a resolution of $0.4 \mathrm{~mm}$ at the SID. Elekta multi-gain panel calibrations for $6 \mathrm{X}$ and $10 \mathrm{X}$ were performed on all panels prior to the Fraction 0 calibration process, which converts pixel intensity to dose. Gamma criterion of $2 \% / 2 \mathrm{~mm}$ was used for checking Fraction 0 calibration and gamma criterion of $3 \% / 2 \mathrm{~mm}$ was used for IMRT/VMAT QA analysis.

Results The 6X multi-gain panel calibrations were repeated several times on PA6 due to poor gamma pass rates (less than 94\%) of the Fraction 0 calibration. In contrast, the gamma pass rate of Fraction 0 
calibration on PA2/ PA5 were all above $98 \%$ for both $6 \times$ and $10 \times$. Though the calibration passed, the $10 \mathrm{X}$ multi-gain panel calibration was repeated on PA2 due to a failure within panel subsections. 2D array measurement with plastic water on PA6 and PerFRACTION measurement on PA2/PA5 showed good pass rate for those IMRT/ VMAT failed PerFRACTION measurement on PA6, indicating the failure lay solely in the PA6 panel, and was not due to a linear accelerator delivery error.

Conclusion Baseline reference fields and corresponding gamma pass rate are useful to check the performance of the EPID panels. PerFRACTION commissioning across three Elekta Agility linear accelerators found the panel response can differ significantly. PerFRACTION 2D analysis of Fraction $0^{\mathrm{TM}}$ has been commissioned on PA2/PA5 for clinical use.

\section{P029 3D Analysis comparison between DoseCHECK ${ }^{\mathrm{TM}}$ and Fraction $0^{\mathrm{TM}}$ in SunCHECK ${ }^{\mathrm{TM}}$ software}

\section{Wawrzak, S F Liu, P Ramachandran}

${ }^{1}$ Radiation Oncology, Cancer Services, Princess Alexandra Hospital, Australia. (Michal.Wawrzak@health.qld.gov.au [Presenting author]), (SauFan.Liu@health.qld.gov.au),

(Prabhakar.Ramachandran@health.qld.gov.au)

Introduction SunCHECK ${ }^{\mathrm{TM}}$ from Sun Nuclear Corporation is a software package offering tools for EPID and/or log file-based patient-specific quality assurance. SunCHECK's DoseCHECK ${ }^{\mathrm{TM}}$ compares dose distribution calculated by TPS against contour-based 3D dose calculation algorithm and can be used as preliminary checks before QA session, indicating eventual plan delivery issues. SunCHECK's Fraction $0^{\mathrm{TM}}$ reconstructs dose based on log files and/ or EPID image obtained from Linac and compares it against the planning system. The aim of this study is to establish the dose agreement of DoseCHECK and Fraction 0 against the TPS.

Method Treatment plan datasets of 156 VMAT cases were exported to SunCHECK server. EPID images were acquired on three treatment machines. 3D gamma was evaluated using $3 \% / 2 \mathrm{~mm}$ gamma criterion in DoseCHECK and log-based Fraction 0. Correlation was established using two-tailed Wilcoxon signed-rank test with significance level of 0.05. 3D gamma threshold of $95 \%$ was examined against treatment machines and treatment sites (abdomen, breast, lung, chest wall, extremities, head \& neck, pelvis \& spine).

Results 3D gamma pass rate was more than $95 \%$ for all machines and treatment sites. Median 3D gamma values for DoseCHECK ${ }^{\mathrm{TM}}$ and Fraction 0 was $99.11 \%$ and $99.16 \%$, respectively. Four of the studied patients had bolus not included in the external contour resulted in failures but upon contouring the bolus, all four cases passed the set gamma criteria.

Conclusion Overall the 3D gamma rate was consistent between DoseCHECK and Fraction 0. Despite a slightly low median gamma values reported by DoseCHECK, it proved to a very good predictor of dose calculation with log-based Fraction 0. As SunCHECK calculation are based on contours as opposed to Pinnacle, any immobilisation devices or scanned in bolus are identified as potential sources of error if not contoured prior to exporting the TPS datasets to SunCHECK.
References/Acknowledgements

Miften M, Olch A, Mihailidis D, et al. Tolerance limits and methodologies for IMRT measurement-based verification QA: Recommendations of AAPM Task Group No. 218. Med Phys. 2018;45(4):e53-e83.

\section{P030 Patient-specific QA for IMRT/VMAT treatment plans: a retrospective analysis of six years' experience}

\author{
Y Yousif, W-L Hsieh, J Daniel, J Zifodya
}

North West Cancer Centre, Tamworth, Australia. (Yousif.Yousif@health.nsw.gov.au [Presenting author]). (WenLong.Hsieh@health.nsw.gov.au), (John.Daniel@health.nsw.gov.au), (Jackson.Zifodya@health.nsw.gov.au)

Introduction The introduction of IMRT and VMAT have promoted performing Patient-Specific QA (PSQA) for each patient before treatment. Several published papers and guideline reviews have addressed the issues of PSQA as well as highlighted its importance. We performed a retrospective analysis of our six years' experience with IMRT/VMAT QA using the ArcCHECK (Sun Nuclear, Melbourne, FL) diode array dosimetry system and reported our departmental guidelines and action levels for IMRT/VMAT delivery. Methods Between March 2014 and March 2020, 318 patients with prostate, head and neck $(H \& N)$, brain, pelvis, and other cancer sites were treated with IMRT/VMAT technique at our institution. Following our departmental protocol, for each patient, PSQA is performed prior to treatment. On Varian Eclipse TPS, all clinical plans are recalculated on the ArcCHECK phantom for verification. Following delivery, measured doses are compared to the calculated doses exported from TPS using the SNC patient Software as well as measured doses using a $0.016 \mathrm{~cm}^{3}$ a 3D pinpoint ionization chamber (PTW-Freiburg). Agreements between measured and calculated doses were evaluated using $3 \%$ (global) $/ 3 \mathrm{~mm}$ and $95 \%$ gamma pass (GP) rate. The mean GP and standard deviations for different sites were evaluated to develop local confidence and tolerance limits.

Results For gamma analysis, the average GP rates were $99.3 \pm 1.0 \%$ for prostate, $98.3 \pm 2 \%$ for $\mathrm{H} \& \mathrm{~N}$ and brain, $98.9 \pm 1.2 \%$ for pelvis. The overall average GP rate was $98.7 \pm 1.5 \%$ and the local confidence limit was determined to be $4.0 \%$.The mean point dose differences and standard deviations were $0.4 \pm 1.0 \%$ for prostate, $-0.4 \pm 0.9 \%$ for brain, $1.2 \pm 1.0 \%$ for pelvis, and $1.0 \pm 1.2 \%$ for $\mathrm{H} \& \mathrm{~N} \&$ other sites. Almost all the point dose results were within our $3 \%$ tolerance limit, except for a few variances.

Conclusion The study showed that the ArcCHECK dosimetry system permits accurate IMRT/VMAT PSQA. Overall our results indicated that our local confidence limits were in-line with the recommended published literature ${ }^{1,2}$.

\section{References}

1. Ezzell GA, Burmeister JW, Dogan N, LoSasso TJ, Mechalakos JG, Mihailidis D, Molineu A, Palta JR, Ramsey CR, Salter BJ, Shi J, Xia P, Yue NJ, Xiao Y: IMRT commissioning: Multiple institution planning and dosimetry comparisons, a report from AAPM Task Group 119. Med Phys 2009, 36:5359-5373. 
2. Mijheer B, Georg D: Guidelines for the verification of IMRT. Brussels, Belgium: ESTRO; 2008.

\section{P031 A sensitivity analysis on calculation and plan QA parameters for VMAT SBRT plans}

\author{
S P F Yung', R Hill ${ }^{2}$ \\ ${ }^{1}$ The University of Sydney, Institute of Medical Physics, School \\ of Physics, NSW 2006, Australia. (felixyung92@hotmail.com \\ [Presenting author]). ${ }^{2}$ Department of Radiation Oncology, Chris \\ O’Brien Lifehouse, Camperdown NSW 2050, Australia
}

Introduction Stereotactic Body Radiation Therapy (SBRT) uses IMRT or VMAT delivery for advanced dose sculpting [1]. The radiotherapy plan requires an extensive set of QA testing. This QA involves comparing calculated and measured dose distribution in a suitable phantom and independent dose calculations. This work examines differences in doses by looking at the sensitivity of different parameters in both planning and QA process.

Method IMRT and VMAT phantom plans were taken from dosimetry audits on a CIRS lung phantom. The Varian Eclipse TPS V15.6 was used for dose calculations using both the AAA and ACUROS algorithms for dose grid sizes ranging from 1.0 to $5.0 \mathrm{~mm}$. Dose comparisons were performed using the Sun Nuclear patient QA and CERR software packages. Different gamma analysis options were analysed to find the most effective options in determining dose differences.

Results IMRT and VMAT calculations showed a negligible difference in DVH for PTV, OAR receives 5\% increase in dose in IMRT compared to VMAT. $1 \mathrm{~mm}$ calculation grid size with AAA produces a higher dose than $5 \mathrm{~mm}$ grid size in PTV and lower dose in OAR. AAA results for PTV and OAR were $1.9 \%$ and $3 \%$ more than ACUROS algorithm respectively. Threshold in gamma analysis could lead to an increase or decrease depending on the passed or failed dose point being excluded. Increasing the value in tolerance would always lead to an increase in pass rate.

Conclusion The VMAT plans reduce the dose to OAR better than IMRT plans. The result showed the dose in AAA for both PTV and OAR are higher than in AXB, and smaller grid size demonstrated a better performance to both volumes. Increasing the tolerance will increase the pass rate for gamma analysis but changing the threshold do not provide a consistent trend with the pass rate.

References

1. Stroom J, Vieira S, Mateus D, Greco C, Fogliata A, Nicolini G, et al. On the robustness of VMAT-SABR treatment plans against isocentre positioning uncertainties. Radiat Oncol. 2014;9(1):1-9.

\section{P032 Translating patient movement to target displacement in Gamma Knife Icon treatment - an initial modelling study}

\author{
H Sandrstöm ${ }^{1}$, C E Jones ${ }^{2}$, P Ramachandran² \\ ${ }^{1}$ Stockholm University and Radiumhemmets forskningsfonder, \\ Sweden. (helena.sandstrom@ki.si). ${ }^{2}$ Radiation Oncology, Princess \\ Alexandra Hospital, Brisbane, Australia. \\ (catherine.jones3@health.qld.gov.au [Presenting author]), \\ (prabhakar.ramachandran@health.qld.gov.au)
}

Introduction Gamma Knife Icon provides stereotactic radiosurgery treatments. Patients are fixated with a thermoplastic mask, and motion during treatment is monitored via its infra-red high definition motion management (HDMM) of a marker placed on the patient's nose. The aim of this study was to evaluate the movement of patients during treatment, and to correlate this movement to the displacement of the target volumes during treatment.

Method Three patients presented with multiple metastases were chosen as an initial dataset. Target displacement was analysed via log-files generated by the HDMM system. The patient movement is transformed to the movement of the target volumes by applying matrix transformation (rotations and translations) to the target volumes, exported in the DICOM format, corresponding to the transformation from reference point to deviation point. The rotation pivot point was identified as a fixed point at the back of the skull by a radiation oncologist. Furthermore, the direction of movement was also evaluated and compared between patients to evaluate a possible trend in the shift.

Results Initial results indicate that the modelling of the patient movement is highly dependent on several factors, particularly location of the pivot point and assumptions regarding the relative contribution of rotation and translation to target displacement. The measured target motion varied for the three patients evaluated.

Conclusion Several factors impacted the results and they should be further evaluated - mainly the selection of the pivot point and the relative contirubtions of rotations and translations applied. However, the initial results show that a substantial relative displacement could occur in fractionated stereotactic radiosurgery treatments, especially for small tumour volumes. Time of displacement also needs to be incorporated for reasonable results.

\section{P033 Varian eclipse 5-millimetre cone data commissioning with a microdiamond detector}

\section{Lew, P Fogg, P Christiansen}

Medical Physics Registrar, Genesis Care, Buderim QLD, Australia. (dominika.lew@genesiscare.com [Presenting author]), (penny.fogg@genesiscare.com), (peter.christiansen@genesiscare.com)

Introduction Conical collimators are effective and readily available accessories for the field shaping of small stereotactic fields, however the measurements required to accurately characterise the smallest radiation fields are difficult and prone to large errors. Furthermore, there is little published commissioning data to compare measurements against. The aim of this investigation was to measure the required Eclipse algorithm data, being tissue maximum ratios (TMR), off axis ratios $(\mathrm{OAR})$ and the output factor $(\mathrm{OF})$ for a 5-millimetre conical collimator attached to a Varian TrueBeam linear accelerator using a microdiamond detector, and compare these to SRS diode measurements, Varian Golden Beam Data (GBD), an ACDS audit measured $\mathrm{OF}$, radiochromic film measurements and the Varian Eclipse treatment planning system (TPS) calculated data.

Method A PTW BEAMSCAN water tank phantom was used to collect the PTW60019 microdiamond data, with the output correction factor derived from TRS-483 [1]. These were compared to the PTW60018 SRS diode measurements, GBD and an ACDS OF. Gamma analysis was used to compare the measured TMRs and profiles, as well as the Eclipse dose planes to the scanned films.

Results It was found that most of the data agreed well, with the microdiamond OF being preferred over the SRS diode OF for the TPS configuration due to the availability of a published small field correction factor in TRS-483 compared to an unpublished correction 
factor for the SRS Diode [1]. In addition, the microdiamond OF determination did not require the daisy chaining method which potentially adds additional error, and the good agreement between the ACDS OF and the microdiamond OF increased confidence in this measurement. The microdiamond detector exhibited slightly increased volume averaging thus the SRS diode TMR and OAR data was retained in the TPS.

Conclusion The main errors encountered concerned the resolution of the Eclipse dose calculation algorithm and FilmQA Pro analysis software. Measurements and analyses were completed successfully for the commissioning of the $5 \mathrm{~mm}$ cone.

\section{References/Acknowledgements}

1. International Atomic Energy Agency (2017) TRS-483 Dosimetry of Small Static Fields Used in External Bram Radiotherapy: An International Code of Practice for Reference and Relative Dose Determination. Med Phys. https://doi.org/10.1002/mp.13208

\section{P034 Evaluation of multi-atlas method for magnetic resonance imaging (MRI)-only prostate proton treatment planning: comparison of lateral opposed and anterior oblique beam arrangements}

\author{
S Hickey ${ }^{1}$, A L Fielding 1 , J A Dowling, 2,3 P B Greer ${ }^{3,4}$, J Seco ${ }^{5,6}$ \\ ${ }^{1}$ Faculty of Science and Technology, Queensland University \\ of Technology, Brisbane. (samantha.hickey@connect.qut.edu.au), \\ (a.fielding@qut.edu.au [Presenting author]). ${ }^{2}$ CSIRO Australian \\ e-Health Research Centre, Herston, Queensland, Australia. \\ ${ }^{3}$ University of Newcastle, Callaghan, New South Wales, Australia. \\ (Jason.Dowling@csiro.com.au). ${ }^{4}$ Calvary Mater Newcastle Hospital, \\ Waratah, New South Wales, Australia. \\ (peter.greer@newcastle.edu.au). ${ }^{5}$ Biomedical Physics in Radiation \\ Oncology, DKFZ - Deutsches Krebsforschungszentrum, Heidelberg, \\ Germany. ${ }^{6}$ Department of Physics and Astronomy, Heidelberg \\ University, Germany. (j.seco@dkfz-heidelberg.de)
}

Introduction As opposed to CT, MRI offers a superior soft tissue contrast, which is favourable for organ or target delineation in radiotherapy. To obtain the benefits of MRI, while eliminating the planning $\mathrm{CT}$ and removing the $\sim 2 \mathrm{~mm}$ uncertainty introduced by CT-MRI registration, an MRI-only workflow was developed. Already a reality in photon therapy, and promising to one day be used in combination with image guided radiotherapy, the MR-only workflow replaces a conventional CT with a synthetic-CT (s-CT) for treatment planning. Proton therapy, however, is more sensitive to uncertainties associated with s-CTs. Of the many applications of an MRI-only proton treatment planning, we focused on prostate cancer, where lateral opposed beams at $90^{\circ}$ and $270^{\circ}$ are currently used; however, an alternate beam arrangement using anterior oblique beams has demonstrated benefits. Furthermore, this study utilities a multi-atlas method for generating an s-CT, developed by Dowling et al. [1], to compare the lateral opposed and anterior oblique beam arrangements. Method This study uses patient data and s-CT generation method from previous work by Dowling et al. [1]. Each patient had a CT and $\mathrm{s}-\mathrm{CT}$ that was used for treatment planning in matRad [2]. Two treatments plans per patient were generated: lateral opposed fields at 90 and 270 degrees and anterior oblique fields at 30 and 330 degrees. Methods of evaluation were DVH, the dose MAE within main contours, and the range shift of the ten central slices.

Results The dose MAE in the PTV was 0.0185 and 0.0201 Gy for anterior oblique and lateral opposed, respectively. All DVH plots were clinically compliant. Less range shift was recorded for the anterior oblique beam arrangement.
Conclusion This study examined differences between the lateral opposed and anterior oblique beam arrangements and demonstrates that an MRI-only workflow for proton prostate treatment is feasible using a multi-atlas method of s-CT generation.
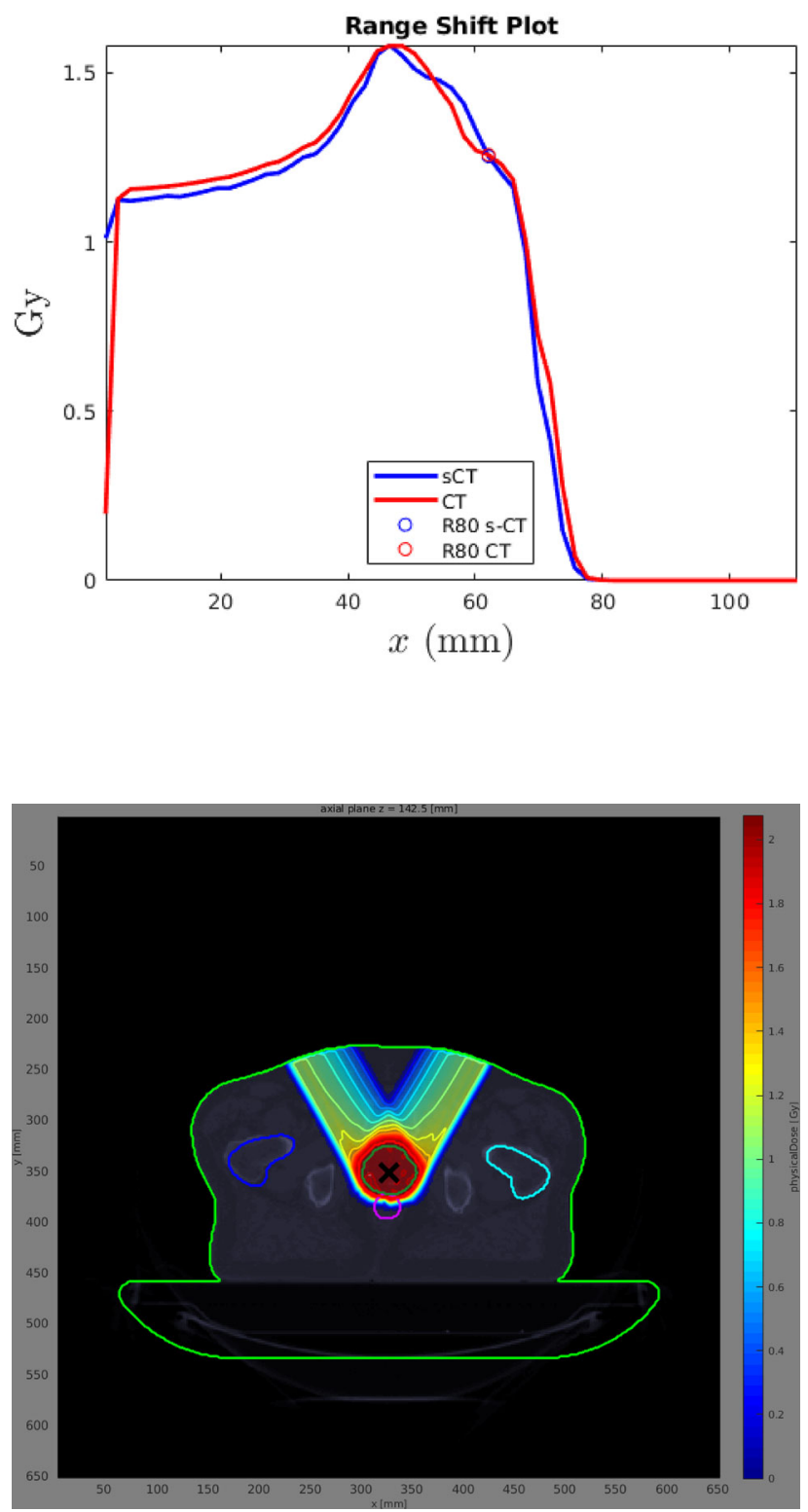

\section{References}

1. J. A. Dowling, J. Sun, P. Pichler, D. Rivest-Hnault, S. Ghose, H. Richardson, C. Wratten, J. Martin, J. Arm, L. Best, S. S. Chandra, J. Fripp, F. W. Menk, and P. B. Greer, International Journal of Radiation Oncology*Biology*Physics 93, 1144 (2015).

2. M. Bangert, A. B. A. Bennan et al. "matRad, e0404, Division of Medical Physics in Radiation Oncology, German Cancer Research Center DKFZ," http://e0404.github.io/matRad/ (2020). 


\section{P035 A National CT curve for treatment planning within GenesisCare}

\section{J P Morton, C Colyer, M Wanklyn, B Subramanian}

Medical Physics, GenesisCare, South Australia. (jason.morton@genesiscare.com), (christopher.colyer@genesiscare.com), (Mark.wanklyn@genesiscare.com), (Brindha.Subramanian@genesiscare.com)

Introduction This talk discusses differences in $\mathrm{CT}$ to density and Electron density curves used for treatment planning across a range of treatment planning systems and CT scanners. It also highlights the differences encountered nationally between phantom size and manufacturer. Results of similar investigations have been published ${ }^{1,2}$, but have not included a range of CT scanners and phantoms used in different departments.

Method CT to Density or electron density curves for a range of treatment planning systems and CT scanners across GenesisCare in Australia were collected. CT scanner response curves were compared across a $\mathrm{kV}$ range of $120 \mathrm{kV}$ to $135 \mathrm{kV}$ with a range of reconstruction algorithms used clinically.

Points of difference were identified and investigated.

Results Three main areas of difference identified were the choice of manufacturer (CIRS 062M Vs Gammex 467), small vs large body phantom, and number of bits of the CT scanner (12 Vs 16 bit).

Conclusion Across a range of $\mathrm{CT}$ scanners and phantoms, the main difference in CT to Density or electron density curves is not only limited to the choice of CT scanner or choice of $\mathrm{kV}$ above $120 \mathrm{kV}$, but also to the choice of $\mathrm{CT}$ to ED phantom manufacturer.

The use of CT scanners in extended range (for 12 and 16 bit scanners) does not accurately reflect the density for materials with HU greater than $2850 \mathrm{HU}$. Hence materials must be considered metal and overridden with the correct density of the material used.

References/Acknowledgements

1. Nakao et al. Med Phys. 2020 Apr;47(4):1509-1522. doi:https:// doi.org/10.1002/mp.14077. Epub 2020 Feb 29.

2. Yohannes et al. Phys Med Biol. 2012; 57:1173-1190. https:// doi.org/10.1088/0031.

\section{P036 Accelerating radiological pathlength calculation using parallel computing}

\author{
K Yin ${ }^{1}$, H Suominen ${ }^{1,2,3}$, E Daskalaki ${ }^{1}$, J Selvaraj ${ }^{4,5,6}$ \\ ${ }^{1}$ Research School of Computer Science, Australian National \\ University. ${ }^{2}$ Data61, Commonwealth Scientific and Industrial \\ Research Organisation. ${ }^{3}$ Department of Future Technologies, \\ University of Turku. ${ }^{4}$ I-Cube Lab, JCSMR, Australian National \\ University. ([Presenting author]). ${ }^{5}$ Faculty of Medicine, University \\ of New South Wales. ${ }^{6}$ Medical Physics \& Radiation Engineering, \\ Canberra Health Services
}

Introduction Radiotherapy planning requires radiological path length (RPL) calculation by ray tracing techniques to account for tissue heterogeneity in dose calculation. A patient's 3D model is generated from set of $2 \mathrm{D}$ cross-sectional CT image slices. The RPL of each voxel is calculated with its relative electron density $\left(\rho_{e}\right)$ which is a function of Hounsfield Unit (HU) that can be obtained from patient's CT image. This is a per-voxel operation. This needs to be done for each beam in the plan. The number of voxels in a 3D model used in planning is very large, so sequential calculation is inefficient. An OpenMP [1] based parallel computing method has been presented in this work.
Method HU to $\rho_{\mathrm{e}}$ relationship was obtained from Pinnacle TPS. The CT image was resampled to $3 \mathrm{~mm}$ isotropic spacing. A ray was created at source position for given beam and traced through all voxels using classic Siddon's raytracing algorithm [2]. The algorithm treated a 3D CT image as a set of orthogonally placed parallel planes as shown in Figure 1. A sequential RPL was already available in our in-house developed software [3]. The nested for-loop was parallelized using OpenMP multithreading. The results were tested on virtual and anonymized CT images from different anatomical sites.

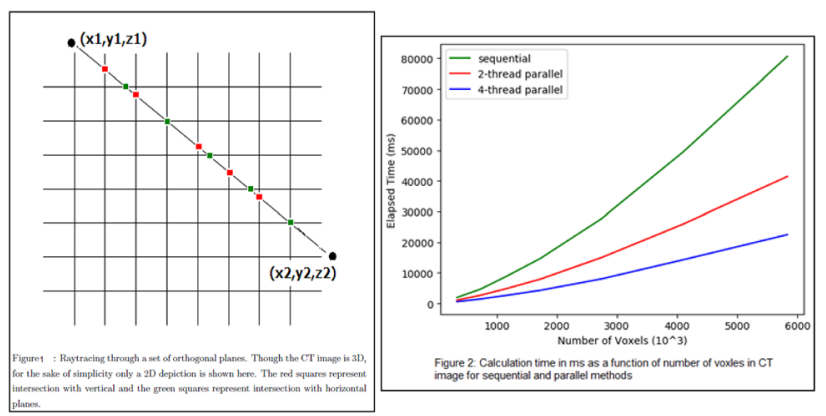

Results Improvement in calculation speed was approximately 3.5 with a 4-core CPU. Furthermore, we found the accuracy of results is around $1 \%$ compared to sequential method which was within clinically acceptable tolerance limits. The differences in calculation speed for sequential and parallel computing methods as a function of number of voxels are shown in Figure 2.

Conclusion A parallel computing method was developed for calculation of RPL which results in faster dose calculation and optimization resulting in higher throughput in TPS. This method was incorporated in in-house developed software for radiotherapy research purposes.

References

1. Dagum, L., \& Menon, R. (1998). OpenMP: an industry standard API for shared-memory programming. IEEE Computational Science and Engineering, 5(1), 46-55.

2. RL Siddon (1985). Fast calculation of the exact radiological path for a three-dimensional CT array. Medical Physics, 12(2), 252-5.

3. Selvaraj, J. K. (2013). Modelling the effect of geometric uncertainties, clonogen distribution and IMRT interplay effect on tumour control probability (Doctoral dissertation, University of Liverpool, Liverpool, UK).

\section{P037 Absorbed dose determination for a mouse phantom using Monte Carlo simulations}

\section{Amir Entezam ${ }^{1,2}$, Roberta Mazzieri ${ }^{3}$, Davide Moi $^{3}$, Chelsea Kralik $^{1,2}$, Davide Fontanarosaa ${ }^{1,2}$, Andrew Fielding ${ }^{2,4}$}

${ }^{1}$ School of Clinical Sciences, Queensland University of Technology, Brisbane, QLD 4000, Australia. ${ }^{2}$ Institute of Health \& Biomedical Innovation, Queensland University of Technology, Brisbane, QLD 4059, Australia. (Amir.entezam @ hdr.qut.edu.au). ${ }^{3}$ Diamantina Institute, Translational Research Institute, The University of Queensland, Woolloongabba, QLD 4102, Australia. (r.mazzieri@uq.edu.au), (d.moi@uq.edu.au). ${ }^{4}$ Chemistry Physics Mechanical Engineering, Queensland University of Technology, Brisbane, QLD 4000, Australia. (a.fielding@qut.edu.au), (chelsea.kralik@hdr.qut.edu.au),(d3.fontanarosa@qut.edu.au) 


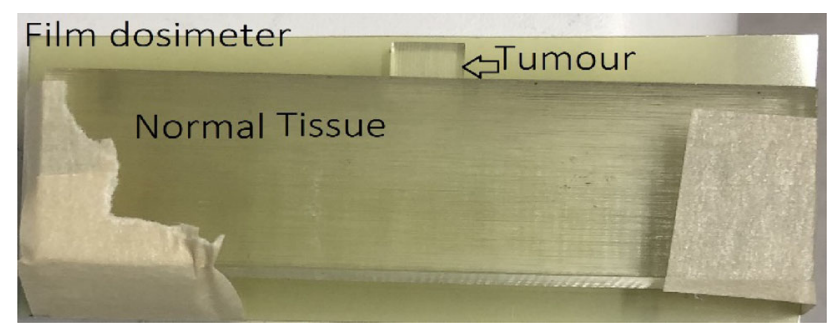

Image 1 Polymethyl Methacrylate (PMMA) phantom

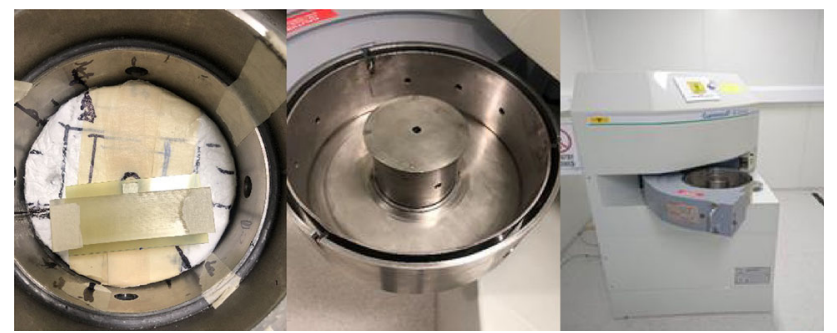

Image 2 Phantom was placed in the collimator and was irradiated using a Cs-137 gamma irradiator

Purpose The objective of this work was to investigate the absorbed dose in a breast tumour and in neighbouring normal tissue of a mouse by means of a water-equivalent phantom and using Monte Carlo (MC) simulations.

Method The mouse phantom was made of Polymethyl Methacrylate (PMMA) to mimic the characteristics of tissue and approximated tumour shape [See Image 1]. The phantom was placed in a specially designed collimator [See Image 2] designed to enable a targeted irradiation using a Cs-137 gamma irradiator [1]. The phantom was irradiated targeting the tumour with doses of 5 to $20 \mathrm{~Gy}$. EBT-XD GAFchromic films were used to measure the dose distribution within the phantom. Additionally, the DOSXYZnrc code was used to compute the $3 \mathrm{D}$ dose distribution in the mouse phantom for comparison with the film measurements.

Results The dose to normal tissue measured with the film detectors was between 8 to $9 \%$ of the tumour dose. Film measurements agreed with the Monte-Carlo calculations to within $2 \%$.

Conclusion This physical phantom is a useful tool to examine individual tumour dose and normal tissue dose of a mouse.

References

1. Fontanarosa, D., Benitez, J., Talkhani, S., Fielding, A., Entezam, A., Trapp, J., ... \& Mazzieri, R. (2020). A novel add-on collimator for preclinical radiotherapy applications using a standard cell irradiator: design, construction, and validation. Medical Physics.

\section{P038 Developing a pathway for Mouse irradiation studies using a clinical superficial treatment unit}

\author{
Tim Markwell ${ }^{1}$, Lucy Sim ${ }^{2}$, Sylwia Zawlodzka-Bednarz ${ }^{1}$, Gishan \\ Ratnayake ${ }^{1}$
}

${ }^{1}$ ROPART - Radiation Oncology Princess Alexandra Hospital Raymond Terrace, Brisbane, Australia.

(Tim.Markwell@health.qld.gov.au [Presenting author]),
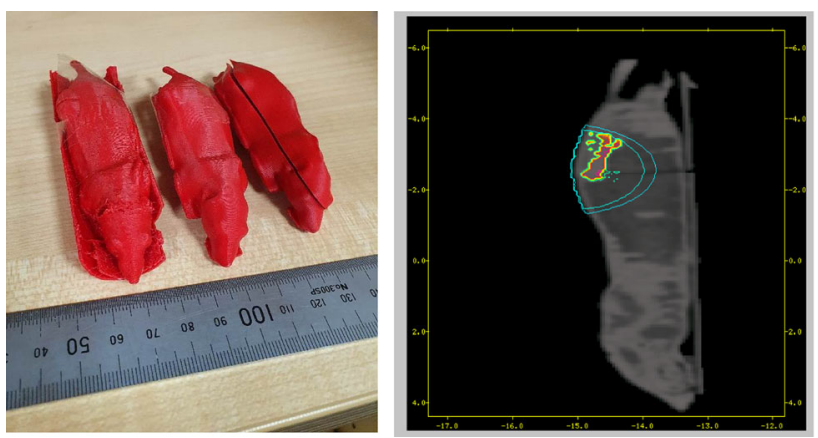

Figure 1 Photo of 3D printed Mice phantoms with splits for film (left), Monte Carlo simulation using a superficial treatment beam (right)

(Lucy.Sim@health.qld.gov.au), (Sylwia.Zawlodzka-

Bednarz@health.qld.gov.au), (Gishan.Ratnayake@health.qld.gov.au)

Introduction Current studies investigating the application of radiotherapy treatments of mice use a wide variety of irradiation techniques. Some techniques irradiate a large percentage of the mouse volume, which can influence the accuracy of the study due to biological effects that differ from a more targeted treatment that would exist with human patients. The aim of this study was to work towards a research platform that can use more targeted radiotherapy to better emulate patient type treatments using mouse models.

Method A micro CT of a mouse with a flank tumour was segmented and converted to a 3D printable file (STL) using Slicer [1]. Multiple phantoms were created and 3D printed using PLA filament. Both homogenous water equivalent phantoms, and phantoms with a skeleton of higher infill density printed in-place were created with splits in the model to allow film placement in the tumour area.

A treatment plan of the beam geometry was generated on custom code and McToolkit [2] for Monte Carlo simulations based on clinical CT of the printed phantoms. The dose distribution from Dosxyz/ BeamNRC [3] using a previously commissioned superficial model of an Xstrahl 150 treatment unit and compared with film measurements. Results and Conclusion The process of taking a Micro CT of a mouse with a tumour $\rightarrow$ 3D printed phantom $\rightarrow$ Monte Carlo simulation, and measurements using film have been demonstrated. Further work to optimise geometry and treatment types depending on the tumour location can now be performed with the goal towards large scale mouse studies using a clinical superficial treatment unit and $3 \mathrm{D}$ Monte Carlo TPS for planning using free open source software (Fig. 85).

\section{References/Acknowledgements}

1. Kikinis R, Pieper SD, Vosburgh K (2014) 3D Slicer: a platform for subject-specific image analysis, visualization, and clinical support. Intraoperative Imaging Image-Guided Therapy, Ferenc A. Jolesz, Editor 3(19):277-289 ISBN: 978-1-4614-7656-6 (Print) 978-1-4614-7657-3 (Online)

2. MC Tools: Livingstone A: https://github.com/livingag/MCtools

3. Rogers, D.W.O., Faddegon, B.A., Ding, G.X., Ma, C.-M., We, J. and Mackie, T.R. (1995), BEAM: A Monte Carlo code to simulate radiotherapy treatment units. Med. Phys., 22: 503-524. https://doi.org/10.1118/1.597552 
P039 Can 3D printed phantoms accurately model lowdensity lung? Investigating the accuracy of Pinnacle for low density lung calculations using 3D printed phantoms

\section{Tim Markwell, Lucy Sim, Sylwia Zawlodzka-Bednarz}

ROPART - Radiation Oncology Princess Alexandra Hospital Raymond Terrace, Brisbane, Australia.

(Tim.Markwell@health.qld.gov.au [Presenting author]),

(Lucy.Sim@health.qld.gov.au), (Sylwia.Zawlodzka-

Bednarz@health.qld.gov.au)

Introduction With the increase in breath hold type treatments for Lung SABR and Breast treatments, changes in the lung density can influence the accuracy of treatment planning systems. With deep inhalation, coupled with diseased lung, lung densities can drop considerably lower than $0.3 \mathrm{~g} / \mathrm{cm}^{3}$ of "normal" lung. This project aims to investigate the accuracy of the Pinnacle TPS for treatments with lowdensity lung.

Method In lieu of commercially available solutions for low-density lung analogue, 3D printed slabs of PLA with a Gyroid infill pattern of densities ranging from $5 \%$ to $30 \%$ were fabricated in-house. Measurements at depths below lung were performed with an ion chamber and compared with Pinnacle and Monte Carlo simulations using BeamNRC/DOSXYZ [1] and MCTools [2].

Results Firstly, validation was performed by comparing the 3D printed gyroid at $30 \%$ infill to the commercial lung phantom (Gammex, Middleton WI) and results showed agreement.

Measurements and Monte Carlo simulations showed good agreement demonstrating the feasibility of using 3D printed gyroid infill slabs as lung analogues for low lung densities.

There was underestimation of dose under lung in Pinnacle, which varied with density and depth beyond the lung. For example, for the $3 \mathrm{~cm}$ thick, $10 \%$ infill, a difference of $4.4 \%$ to $4.7 \%$ was observed at $1-2 \mathrm{~mm}$ from the lung interface and this difference reduced to $\sim 2 \%$ at $2 \mathrm{~cm}$. Dose in lung, using DVH analysis, also showed a detectable difference between Monte Carlo and Pinnacle.

Conclusion This investigation has shown that 3D printed lung slabs using a gyroid pattern with variable infill can act as a novel lung equivalent substitute for low lung densities. Comparison with Pinnacle, Monte Carlo and measurements has shown a detectable divergence between Pinnacle calculations and the physical transmission. Further work investigating dose to tumour in lung will also be performed in the future (Fig. 86).

References/Acknowledgements

1. Rogers, D.W.O., Faddegon, B.A., Ding, G.X., Ma, C.-M., We, J. and Mackie, T.R. (1995), BEAM: A Monte Carlo code to

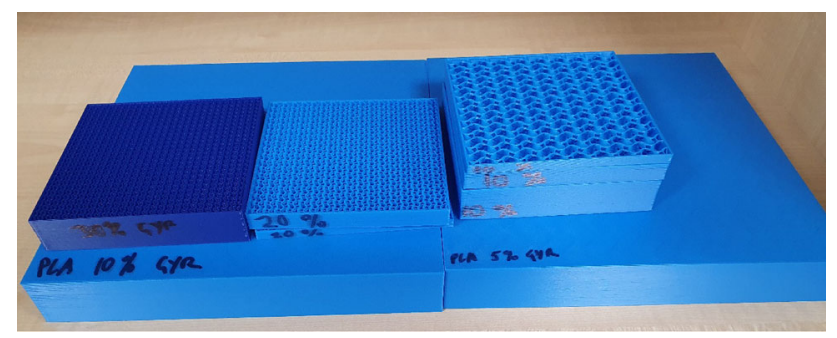

Figure 1 Image showing the 3D printed lung slabs. The large slabs were printed with minimal top and bottom layers to reduce the influence in measurement simulate radiotherapy treatment units. Med. Phys., 22: 503-524. https://doi.org/10.1118/1.597552

2. MC Tools: Livingstone A: https://github.com/livingag/MCtools

\section{P040 Assessment of out of patient plane leakage doses in high energy electron beams for a Varian Clinac iX}

\section{J Miller, L Sim}

Radiation Oncology Princess Alexandra Raymond Terrace, Princess Alexandra Hospital, South Brisbane, Australia. (JulieAnne.Miller@health.qld.gov.au [Presenting author]), (Lucy.Sim@health.qld.gov.au)

Introduction Electron treatment in radiation therapy can necessitate patient setup position such that the non-target tissue is in close proximity to the electron applicator side walls. The IEC [1] specifications state that the applicator side plane leakage should not exceed $10 \%$ of the central axis dose at $D_{\max }$. Measurements were made to determine the amount of leakage through the sides of the applicator and quantify the thickness of lead for effective shielding to protect the lens or skin.

Method A film wrap was initially performed with XRQA-2 on a $10 \times$ $10 \mathrm{~cm}^{2}$ applicator on a Varian Clinac iX to identify hotspots. Measurements using various dosimeters were performed for $6-18 \mathrm{MeV}$ at $5-8 \mathrm{~cm}$ from the projection of field edge. The ionisation ratio and dose were measured using an Advanced Markus chamber and PTW 60017 Diode E respectively and in water equivalent material. Ratios are normalised to $D_{\max }$ at Gantry $0^{\circ}$ and $100 \mathrm{~cm} \mathrm{SSD} \mathrm{(Fig.} \mathrm{87).}$

Results Leakage dosimetry was quantified with an ionisation ratio curve measured using an Advanced Markus for $0-10 \mathrm{~mm}$ and it was comparable to the dose readings acquired using the PTW Diode E. The maximum peripheral leakage from applicator side walls was $<5 \%$ of the central-axis dose maximum, with the highest dose leakage of $3.1 \%$ at $5 \pm 0.5 \mathrm{~mm}$. The ionization ratio and diode dose ratio at $10 \mathrm{~mm}$ depth were within $0.5 \%$. Adding a wax coated piece of $10 \mathrm{~kg} / \mathrm{m}^{2}$ lead lowered the leakage to less than $1 \%$ for all energies. Conclusion The leakage doses from the sides of a Varian iX electron applicator are low and can be effectively reduced further with $10 \mathrm{~kg} /$ $\mathrm{m}^{2}$ lead eye or skin shielding.

\section{References}

1. International Electrotechnical Commission. Medical Electrical Equipment - Part 2-1: Particular requirements for the basic safety and essential performance of electron accelerators in the range 1 $\mathrm{MeV}$ to $50 \mathrm{MeV}$ (2009), IEC 60601-2-1 V3

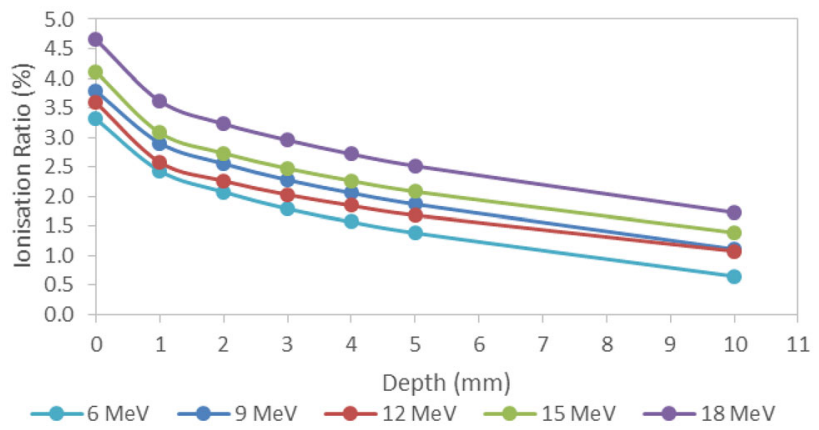

Figure 1 Depth ionisation curve normalised to the central axis Dmax of the leakage from the applicator side wall at $5 \mathrm{~cm}$ from field edge 


\section{P041 Monte Carlo evaluation of surface dose from flattening filter (FF) and flattening filter free (FFF) beams}

\section{Hossain Deloar, J Mathew, V Peng, P Narayanan, T Kron}

Department of Physical Sciences, Peter MacCallum Cancer Centre, Australia. (Deloar.Hossain@ petermac.org [Presenting author]), (Joby.Mathew@petermac.org), (Valery.Peng@petermac.org), (Pradush.Narayanan@petermac.org), (Tomas.Kron@petermac.org)

Introduction Clinical linacs have photon beams with and without flattening filter. Flattening filter in the beam path hardens and flattens the beam and generates secondary electrons, while FFF beams have a softer spectrum of $\mathrm{x}$-rays and electrons. This study evaluates the surface dose and the contribution from electron contamination for 6 MV-FF, 10 MV-FF, 6 MV-FFF and 10 MV-FFF beams using Monte Carlo.

Method A Varian clinac was previously modelled and validated ${ }^{1}$ for $6 \mathrm{MV}$ and $10 \mathrm{MV}$ beams using the EGSnrc/BEAMnrc Monte Carlo (MC) $\operatorname{code}^{2}$. The same MC input files were used for FF beams, while the flattening filter was removed for FFF beams. For each energy, particle information from MC simulations of a $10 \mathrm{~cm} \times 10 \mathrm{~cm}$ field were stored in a phase space file and the fluence, energy spectra and electron contamination were evaluated. Phase space files were then used to calculate PDD in water using the DOSXYZnrc MC code. Surface dose in the build-up region were evaluated for all FF and FFF beams.

Results Significant differences in the energy fluence were observed between FF and FFF beams for both $6 \mathrm{MV}$ and $10 \mathrm{MV}$ (Figure 1). From the phase space files, the amount of electron contamination was $0.30 \%$ for $6 \mathrm{MV}$-FFF, $0.35 \%$ for $6 \mathrm{MV}-\mathrm{FF}, 0.40 \%$ for $10 \mathrm{MV}$-FFF and $0.50 \%$ for $10 \mathrm{MV}-\mathrm{FF}$. PDD calculations showed $15 \%$ higher surface dose for $6 \mathrm{MV}$-FFF compared to $6 \mathrm{MV}$-FF and $25 \%$ higher surface dose for $10 \mathrm{MV}$-FFF compared to $10 \mathrm{MV}$-FF.

Conclusion Significant differences in energy fluence were observed between FF and FFF beams. Electron contamination for FF and FFF beams are similar. The higher surface dose for FFF beams is therefore due to its softer spectrum compared to FF beams.

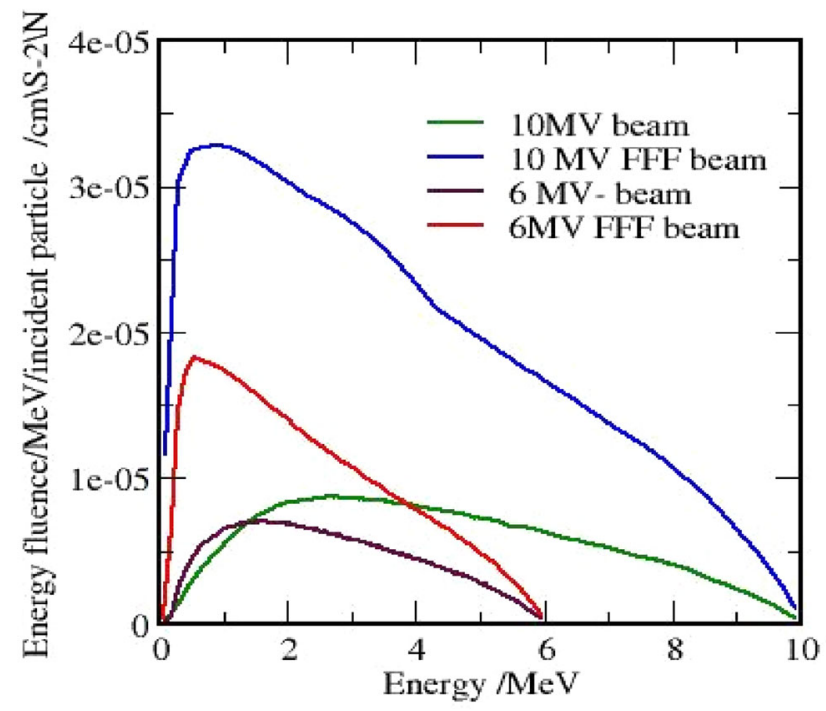

Figure 1 Energy fluence distributions for $10 \mathrm{MV}-\mathrm{FF}, 10 \mathrm{MV}-\mathrm{FFF}, 6$ MV-FF and 6 MV-FFF beams

\section{References}

1. HM Deloar, et al. "Evaluation of Clinical Dose Distributions Using Monte Carlo Methods". World Congress on Medical Physics and Biomedical Engineering 2006 pp 1956-1957

2. D. W. O. Rogers, et al, "BEAM: A Monte Carlo code to simulate radiotherapy treatment units,” Med. Phys. 22, 503-524, 1995.

\section{P043 Occupational dose during CT-guided interventions}

\author{
Yohei Inaba $^{1,2}$, Shin Hitachi ${ }^{3}$, Koichi Chida ${ }^{1,2}$ \\ ${ }^{1}$ International Research Institute of Disaster Science, Tohoku \\ University, Japan. ${ }^{2}$ Graduate School of Medicine, Health Sciences, \\ Tohoku University, Japan. (inaba@med.tohoku.ac.jp [Presenting \\ author]). ${ }^{3}$ Department of Radiology, Tohoku University Hospital, \\ Japan. (hitachi@rad.med.tohoku.ac.jp). (chida@med.tohoku.ac.jp)
}

Introduction The physicians are close to the source of scattered radiation dose during CT-guided interventions. Therefore, dose-reducing/optimizing for physicians are important. Furthermore, the occupational dose limit to the eye lens is reduced approximately $1 / 8$. The physician and medical staff are at high risk of radiation-induced injury, such as cataracts. However, few previous reports have investigated radiation doses to physicians in MDCT-guided interventions. The purposes of this study were to evaluate the radiation dose to the physician and medical staff during CT-guided interventions.

Method The procedures were performed using IVR-CT system. We evaluated the occupational radiation dose (physician and medical staff) and related parameters (fluoroscopy time, mAs, CTDI and DLP) performed consecutive about 200 procedures in CT-guided biopsy.

Results Physician doses (eye lens, neck and hands; $\mu$ Sv, average \pm $\mathrm{SD})$ in our MDCT-guided interventions were $39.1 \pm 36.3,23.1 \pm$ 23.7 , and $28.6 \pm 31.0$, respectively. Medical staff (nurse) doses were lower than the physician doses, obviously. Acquisitions numbers, fluoroscopy time, fluoroscopy mAs (average $\pm \mathrm{SD}$ ) of our MDCTguided interventions were $34.7 \pm 23.1,26.6 \pm 17.8 \mathrm{~s}$, and $650.0 \pm$ 598.4, respectively.

Conclusion We have understand the importance of reducing/optimizing the dose to the physician and medical staff in CT-guided interventions. In our study, the physician and medical staff doses were not significant when the procedures were performed with appropriate radiation protection and low-dose techniques.

References/Acknowledgements as applicable

\section{P046 The radiation dose of single vs dual-phase CT Chest Abdomen Pelvis with contrast in trauma patients}

\author{
M Lawson ${ }^{1,2}$, A Kuganesan', M Badawy ${ }^{1,3}$, \\ ${ }^{1}$ Monash Imaging, Monash Health, Australia. ${ }^{2}$ Centre for Medical \\ and Radiation Physics, University of Wollongong, Australia. \\ (Michael.lawson@monashhealth.org [Presenting author]), \\ (Ahilan.Kuganesan@monashhealth.org). ${ }^{3}$ Department of Medical \\ Imaging and Radiation Sciences, School of Primary and Allied Health \\ Care, Faculty of Medicine, Nursing and Health Sciences, Monash \\ University, Clayton, VIC 3800, Australia. \\ (Mohamed.Badawy@monashhealth.org)
}

Introduction CT chest abdomen pelvis (CAP) studies with contrast deliver some of the highest radiation doses in diagnostic CT. Thus optimising the scan parameters to lower the radiation burden on the 
patient becomes paramount for reducing associated radiation risks. One method of lowering the radiation dose in CT CAP is to conduct the entire scan in a single-phase acquisition, rather than a chest scan followed by an abdomen pelvis scan. The single phase approach reduces the amount of tissue that is scanned in the overlap between the two acquisitions. This study aims to compare the organ dose and effective dose to patients undergoing CT CAP studies using a dualphase approach versus a single-phase approach.

Method A retrospective audit of trauma patients undergoing singlephase CAP and dual-phase CAP on the same CT scanner was conducted. The radiation dose was simulated on CT Expo v2.5, and a student T-test was conducted to assess the significance between both groups.

Results The radiation dose was significantly lower for both male and female patients undergoing a single-phase acquisition. Additionally, the radiation dose to the breast tissue was significantly decreased using the single-phase approach.

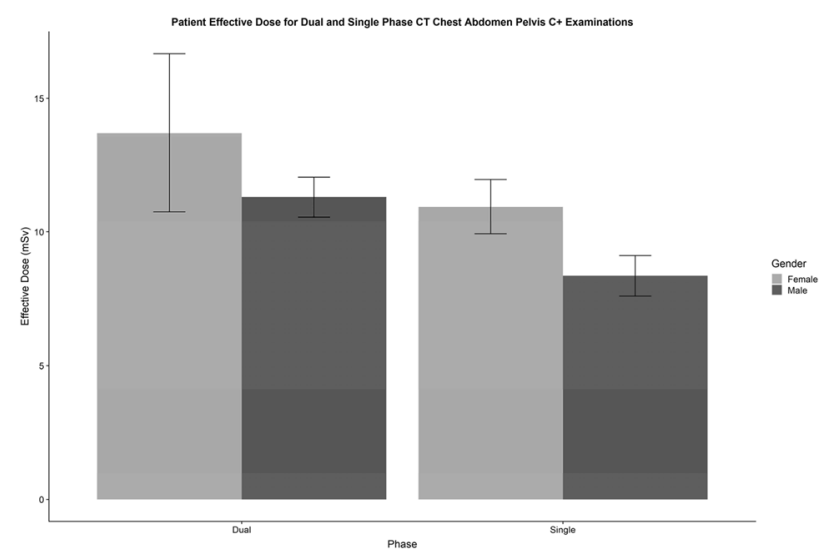

Conclusion Radiation dose optimisation in trauma patients requiring CT CAP with contrast is feasible by utilising a single-phase scanning approach. The dose reduction due to not scanning the same organs twice due to overlap outweighs the absorbed dose due to the slightly increased radiation output through the chest region.

\section{References/Acknowledgements}

1. García-Garrigós, Elena, Juan José Arenas-Jiménez, and José Sánchez-Payá. "Best protocol for combined contrast-enhanced thoracic and abdominal CT for lung cancer: A single-institution randomized crossover clinical trial." American Journal of Roentgenology (2018): 1226-1234.

\section{P047 Establishing Local Diagnostic Reference Levels (LDRLs) for general x-ray imaging at a large specialized paediatric institution}

\section{D.Dinham, E. Ryan}

Biomedical Technology Services, Health Support Queensland, Queensland Health, QLD, Australia.

(dalia.dinham@health.qld.gov.au), (elaine.ryan@health.qld.gov.au [Presenting author])
Introduction Local diagnostic reference levels (LDRLs) are an important tool to support the optimisation of imaging examinations. There are currently no published Australian Diagnostic Reference

Table 1 Age groupings for LDRL data

\begin{tabular}{llll}
\hline Group & $\begin{array}{l}\text { Age groups used } \\
\text { for HEAD exams }\end{array}$ & Group & $\begin{array}{l}\text { Age groups used } \\
\text { for BODY exams }\end{array}$ \\
\hline 1 & $0-<3$ months & 1 & $<1$ month \\
2 & 3 months $-<1$ year & 2 & 1 month -12 months \\
3 & $1-<6$ years & 3 & $>12$ months to 4 years \\
4 & $\geq 6$ years & 4 & $>4$ years to 7 years \\
& & 5 & $>7$ years to 10 years \\
& 6 & 10 years to 14 years \\
\hline
\end{tabular}

Levels for general $\mathrm{x}$-ray examinations for paediatric patients. Local DRLs have been established for the most frequent and relatively higher dose examinations and compared to published international DRLs [1] for the Queensland Children's Hospital (QCH).

Method A total of 110,000 patient exposure records were included in this assessment. The LDRLs have been defined at the $75^{\text {th }}$ percentile for five units in the department grouped together [2]. This paper presents the LDRL values for general radiography for 15 examinations, where there was adequate data, across six age groups, given in Table 1 .

The anatomical regions for which adequate data was available are head, chest, abdomen, pelvis, cervical spine, thoracic spine, lumbar spine and whole spine, with AP/PA and lateral projection data. The dosimetric quantity used to set the LDRLs was the dose area product (DAP) in units of $\mathrm{mGycm}^{2}$.

Results The results of the LDRL analysis are given in Table 2:

Table 2 Results for all projections for LDRL data

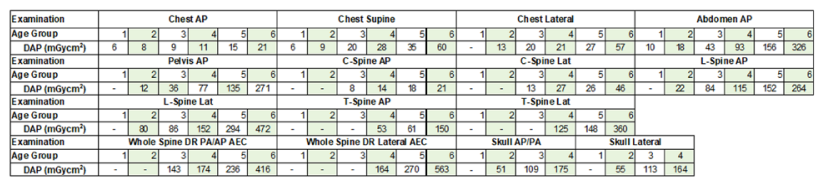

These results have been summarised and compared to published international DRLs. The LDRLs were benchmarked against the DRLS defined by the European paediatric DRLs [1] (EDRLs) where available. The LDRLs for paediatric general x-ray examinations are lower than the published EDRLs for all age groups and available examinations.

Conclusion Establishing LDRLs for general x-ray procedures is useful for providing a baseline for future optimisation of exposure levels. It has shown that QCH has LDRLs that are lower than published European data.

\section{References}

1. Bosmans H, Damilakis,J. Ducou le Pointe H, Foley S, et al (2018) European Diagnostic Reference Levels for Paediatric Imaging, Report Number 185, European Society of Radiology. https//doi.org/https://doi.org/10.2833/003998 
2. Marsden PJ, Hardwick J, Mencik C, McLaren C, Young C, Mashford P. et al, (2001) The establishment and use of dose reference levels in general paediatric radiology (IAEA-CSP-7/P). International Atomic Energy Agency (IAEA)

\section{P048 X-ray artifacts caused by manufacturing defect within neonates incubator unit - a case study}

\author{
S Aliuddin ${ }^{1}$, S Braiden ${ }^{1}, \mathrm{~V} \mathrm{Koi}^{2}$, C Devaney ${ }^{2}$ \\ ${ }^{1}$ Medical Physics, Biomedical Technology Services, Queensland, \\ Australia. (sarah.aliuddin@ health.qld.gov.au [Presenting author]). \\ (samuel.braiden@health.qld.gov.au). ${ }^{2}$ Royal Brisbane \& Women's \\ Hospital, Queensland, Australia. (vicki.koi@ health.qld.gov.au), \\ (carron.devaney@health.qld.gov.au)
}

Introduction There were multiple reports on various artifacts found on x-ray images involving neonates in the incubator unit at the Royal Brisbane \& Women's Hospital. An investigation was initiated to find the origin of these artifacts. From the screening process, it was discovered that these artifacts to be a result of manufacturing defects within the detector tray and scale component of the incubator unit.

Method A systematic approach was used to identify the source of the artifacts on the $\mathrm{x}$-ray images taken of the neonates inside an incubator unit. Firstly, all CR cassettes and other materials commonly used inside the incubator unit, such as blanket and mattresses, were screened to ensure that they are free from artifacts. Secondly, components identified within the X-rays primary beam were dissembled from the main incubator unit and screened to check for foreign materials or defects that may cause artifact appearance. All x-ray images taken from the screening were subjectively analysed as per criteria set by the radiologist.

Results From this investigation, it was discovered that artifacts in the $\mathrm{x}$-ray images of the neonates resulted from manufacturing defects within the detector tray and the scale component of the incubator unit. Conclusion Following this investigation, a plan to screen all incubator units in NICU is put in place to identify the extent of manufacturing defects for TGA $[1,2]$ reporting and isolate any defective components that may cause artifacts in the neonate $\mathrm{x}$-ray images. The fleet screening process will also be performed in stages to minimise the impact on the clinical operation. In this process, all defective components will be returned to the manufacturer, and a replacement will be requested. Medical physics is also encouraged to be involved in the future acceptance process involving medical equipment such as neonates incubator unit before the unit is released into clinical use. This is so the care and safety for each neonate are kept to the highest level by reducing the number of repeat $\mathrm{x}$-rays while maintaining medical diagnosis integrity.

References

1. TGA Health Safety Regulation (2017) Therapeutic product vigilance (V1.4)

2. TGA Health Safety Regulation (2011) The Therapeutic Goods Administration's risk management approach to the regulation of therapeutic goods(V4.0)

\section{P049 The importation of medical radioisotopes into Australia}

Paul Marks, Toby Beveridge, Peter Thomas

ARPANSA, Yallambie, VIC Australia
Background Regulation 4R "Importation of Radioactive Substances" of the Customs (Prohibited Imports) Regulations 1956, as amended, deals with shipments of radioactive material entering Australia. The CEO of ARPANSA or an authorised officer as appointed by the Minister can approve the importation into Australia of a radioactive substance.

A radioisotope is considered to be for medical use when it is intended to be:

1. administered to humans for diagnostic or therapeutic procedures

- used in any in-vitro medical diagnosis or test

- used in research which is either directly or indirectly related to medical diagnosis or therapy

Sealed and unsealed radioactive sources used to calibrate instruments in medical practices and pathology laboratories are also included as medical radioisotopes for permit purposes.

Aims This paper will highlight the methodology that ARPANSA employs to ensure the efficient authorisation of importations of medical radioisotopes into Australia.

Methods Under the Customs (Prohibited Imports) Regulations, a permit from ARPANSA is required for the import of radioactive material. Applications for the import of radioisotopes for medical use must be made to ARPANSA on the appropriate form. These applications can be for either a single shipment or, under certain conditions, for an unlimited number of shipments over 12 months.

Results ARPANSA processes hundreds of permits a year, predominately for medical therapeutic uses in brachytherapy and radiopharmaceutical therapy with unsealed sources. ARPANSA's ability to be flexible when dealing with clients, hospitals and state radiations regulators ensure uninterrupted access to radioisotopes in Australia.

Conclusion The importation of medical radioisotopes is vital for the health and wellbeing of Australian patients. ARPANSA recognises the need for a quick and efficient service that puts the needs of patients at the forefront of the process.

\section{P050 Quantitative clinical governance in orthopaedic surgery using administrative data}

\section{R Smith ${ }^{1}$, D Rimmington ${ }^{2}$, P Weinrauch ${ }^{3}$, M Gillman ${ }^{4}$, J T Rivers ${ }^{5}$}

${ }^{1}$ Physical Sciences, St Andrew's War Memorial Hospital, Brisbane Australia. (Ian.smith@uchealth.com.au [Presenting author]).

${ }^{2}$ Rimmington Orthopaedics, Brisbane, Australia.

(dale@dalerimmington.com.au). ${ }^{3}$ Brisbane Hip Clinic, Brisbane, Australia. (pweinrauch@brisbanehipclinic.com.au). ${ }^{4}$ Medical Services, St Andrews War Memorial Hospital, Brisbane, Australia (Michael.Gillman@uchealth.com.au). ${ }^{5}$ Queensland Cardiovascular Group, Brisbane, Australia. (johnR@qcg.com.au)

Introduction Clinical Governance processes informed by Statistical Process Control (SPC) tools have been shown to promptly flag potentially significant variations in clinical performance in a range of health care settings. Such systems usually rely on data from dedicated clinical registries, however, these are traditionally expensive to establish and maintain. Data from administrative systems is viewed as a viable alternative data source. We discuss the application of these techniques to inform the morbidity and mortality (M\&M) processes in Orthopaedic Surgery at a single site.

Method Routinely collected coding, financial and limited adverse outcome screening (LAOS) data for acute orthopaedic surgical admissions, between January 2013 and the March 2020, were retrospectively evaluated. A range of process and outcome performance 
measures (with risk adjustment) and benchmarks were developed and reported. Exponentially Weighted Moving Average (EWMA) charts and Funnel Plots have been developed for discussion at the unit's routine specialty meetings.

Results Discrete and aggregated measures, including Unplanned Return to Theatre, outlier Length of Stay and Readmission $<30$ days have proven to be usable measures for monitoring clinical performance. While clinical outcome measures based on major morbidities (Death, Neurological events) are valuable, their low event rates pose problems for SPC techniques. Less robust clinical endpoints derived from coding must be used with caution due to the nature of the data source. Use of monitoring tools based around these measures may best be used as a screening tool for identification of cases for detailed review.

Conclusions SPC tools facilitate near "real-time" performance monitoring allowing early detection and intervention in instances of altered performance. While having some limitations by comparison to analysis using prospectively collected clinical registry data, careful and considered review of analysis may prove helpful in detecting and differentiating systemic and individual clinician variation. 